\title{
ACCURATE SYMMETRIC EIGENREDUCTION BY A JACOBI METHOD
}

\author{
DISSERTATION \\ Zur \\ Erlangung des Grades Dr. rer. nat. \\ des Fachbereichs Mathematik \\ der Fernuniversität - Gesamthochschule - Hagen
}

\author{
vorgelegt von \\ IVAN SLAPNIČAR \\ aus Split, Kroatien
}

Hagen 1992 
Erstgutachter und Mentor: Prof. Dr. K. Veselić, Hagen Zweitgutachter: Prof. Dr. J. Demmel, Berkeley 


\section{Acknowledgement}

I would like to thank my mentor Prof. Dr. Krešimir Veselić for introducing me to the exciting field of relative error analysis, for devoting to me a lot of his time, and for sharing with me so many of his ideas.

I also thank my colleagues Eberhard Pietzsch, Zlatko Drmač und Xiaofeng Wang for the possibility to check my ideas in numerous discussions, and Prof. Dr. Jesse Barlow, Pennsylvania State University, for his comments.

Finally, I thank Prof. Dr. James Demmel, University of California, Berkeley, for his valuable and detailed remarks. 
Mojoj obitelji

$æ$ 


\section{Contents}

1 Introduction 3

2 Floating-point perturbations of Hermitian matrices $\quad 11$

2.1 Introduction and preliminaries . . . . . . . . . . . . . . 11

2.2 Well-conditioned scalings . . . . . . . . . . . . . . 15

2.2.1 Perturbation of the eigenvectors . . . . . . . . . . 27

2.3 Perturbations by factors . . . . . . . . . . . . . . 30

2.3.1 Perturbation of the eigenvectors . . . . . . . . . 36

3 Error analysis of the $J$-orthogonal Jacobi methods $\quad 41$

$3.1 J$-orthogonal Jacobi method . . . . . . . . . . . . . . . . . . . . 41

3.2 Error bounds for the eigenvalues . . . . . . . . . . . . . . . . . . . . . . . . . . . . . . 45

3.2.1 The modified method . . . . . . . . . . . . . . . 63

3.2.2 Growth of the condition of the scaled matrix . . . . . . . . 66

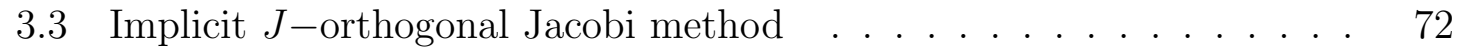

3.3.1 Keeping the diagonal in a separate vector . . . . . . . . . . 83

3.3.2 Error bounds for the eigenvectors . . . . . . . . . . . . . 86

3.4 Fast implicit method . . . . . . . . . . . . . . . . . . . . 89

3.4 .1 Self-scaling rotations . . . . . . . . . . . . . 98

4 Symmetric indefinite decomposition $\quad 103$

4.1 Introduction and algorithm . . . . . . . . . . . . . . . . 103

4.2 Error analysis . . . . . . . . . . . . . . . . . . . . . . 109

4.3 Overall error bounds . . . . . . . . . . . . . . . . . . . . . 117

4.4 Bound for the scaled condition of $G^{T} G \ldots \ldots \ldots$

5 Numerical experiments $\quad 131$

$\begin{array}{ll}\text { Bibliography } & 141\end{array}$ 


\section{Chapter 1}

\section{Introduction}

In this thesis we consider the eigenvalue problem

$$
H x=\lambda x, \quad x \neq 0
$$

where $H$ is a real symmetric matrix of order $n$. Our aim is the following:

if the matrix is "well-behaved", that is, if small relative changes of the matrix elements cause small relative changes in the eigenvalues, then perform the eigenreduction accurately in this sense.

Our work generalizes the works by Barlow and Demmel [2] who considered scaled diagonally dominant matrices (which are described later), and by Demmel and Veselić [13] who considered positive definite matrices. Our results are, however, less definite than in the positive definite case. This is due to the fact that the structure of the set of all well-behaved indefinite matrices is more complicated than the structure of the set of all well-behaved positive definite matrices, and is not simply characterized as yet. Demmel and Veselić's [13] algorithm of choice was the Jacobi method. One of the versions of the algorithm that they used consists of two steps. First step is to calculate the Cholesky decomposition of a starting positive definite matrix. Second step is to apply the implicit (one-sided) version of the Jacobi method to the Cholesky factor as described by Veselić and Hari [31]. We use the algorithm which is an immediate generalization of this two-step algorithm, and was proposed by Veselić [28, 29].

The algorithm consists of two steps.

1. Decompose $H$ as

$$
H=G J G^{T}, \quad J=I_{\text {npos }} \oplus\left(-I_{r-n p o s}\right),
$$

where $G$ is a $n \times r$ matrix of a full column rank, $\operatorname{rank}(H)=r$, and npos is the number of the positive eigenvalues of $H$. 
This decomposition is an extension of the known symmetric indefinite decomposition of Bunch and Parlett [6]. The eigensolutions of the matrix $H$ and the pair $G^{T} G, J$ are simply related. There always exists a matrix $F$ which diagonalizes the pair $G^{T} G, J$ such that

$$
F^{T} G^{T} G F=\Delta, \quad F^{T} J F=J .
$$

where $\Delta$ is diagonal and positive definite. The matrices for which $F^{T} J F=J$ are called $J$-orthogonal. The non-zero eigenvalues of $H$ are the diagonal elements of $\Delta J$, and the corresponding eigenvectors are the columns of $G F \Delta^{-1 / 2}$.

2. Apply the implicit (one-sided) $J$-orthogonal Jacobi method to the pair $G, J$ to find the non-zero eigenvalues and the corresponding eigenvectors of $H$.

The implicit $J$-orthogonal Jacobi method consists of an iterative application of the transformation

$$
G_{m+1}=G_{m} J_{m},
$$

where $G \equiv G_{0}$ and $J_{m}$ is a $J$-orthogonal Jacobi plane rotation. The $J$-orthogonality of $J_{m}$ means that $J_{m}$ performs a hyperbolic rotation if $1 \leq i \leq n p o s<j \leq r$, and a trigonometric rotation otherwise. Since the implicit Jacobi works only on the columns of $G$, it is suitable for parallel computing. The symmetric indefinite decomposition (1.1) is, however, not suitable for parallelization. The transition from the matrix $H$ to the pair $G^{T} G, J$ is, in fact, one step of the LR algorithm and usually has a diagonalizing effect. This reduces the number of iterative steps in our algorithm, and makes it faster than the standard Jacobi.

The algorithm has very favourable accuracy properties. For most well-behaved matrices we were able to prove relative error bounds for the eigenvalues and the norm error bounds for the eigenvectors similar to those in [13]. These errors are uniformly better than those for QR or the standard Jacobi algorithm applied directly to $H$.

Now we present our error bounds. They depend on new perturbation theory for eigenvalues and eigenvectors, error analysis of the symmetric indefinite decomposition, and error analysis of the $J$-orthogonal Jacobi methods. The statement that our algorithm is more accurate than QR or the standard Jacobi algorithm depends also on some empirical observations for which we have overwhelming numerical evidence, but somewhat weaker theoretical understanding. Our perturbation theory is an extension of those of Barlow and Demmel [2] and Demmel and Veselić [13].

We first consider known results. Let $H$ be a real non-singular symmetric matrix. Let $\delta H$ be a small symmetric perturbation of $H$ such that

$$
\left|\delta H_{i j}\right| \leq \varepsilon\left|H_{i j}\right| \text {. }
$$

Let $\lambda_{i}$ and $\lambda_{i}^{\prime}$ be the $i$-th eigenvalues of $H$ and $H+\delta H$, respectively, numbered so that $\lambda_{1} \leq \cdots \leq \lambda_{n}$. The standard perturbation theory [33] says that (1.2) implies

$$
\frac{\left|\lambda_{i}-\lambda_{i}^{\prime}\right|}{\lambda_{i}} \leq \frac{\|\delta H\|_{2}}{\lambda_{i}} \leq \varepsilon \sqrt{n}\|H\|_{2} \cdot\left\|H^{-1}\right\|_{2}=\varepsilon \sqrt{n} \kappa(H)
$$


where $\kappa(H) \equiv\|H\|_{2} \cdot\left\|H^{-1}\right\|_{2}$ is the condition number of $H$. For the positive definite $H$, Demmel and Veselić [13] proved the following stronger result: write $H=D A D$ where $D=(\operatorname{diag}(H))^{1 / 2}$ is a scaling so that $A_{i i}=1$. Then (1.2) implies

$$
\frac{\left|\lambda_{i}-\lambda_{i}^{\prime}\right|}{\lambda_{i}} \leq \frac{\varepsilon n}{\lambda_{\min }(A)} \leq \varepsilon n \kappa(A)
$$

By a theorem of Van der Sluis [27]

$$
\kappa(A) \leq n \min _{D} \kappa(D H D)
$$

i.e. $\kappa(A)$ nearly minimizes the condition number of positive definite $H$ over all possible diagonal scalings. Clearly, it is possible that $\kappa(A) \ll \kappa(H)$ and it is always true that $\kappa(A) \leq n \kappa(H)$, so the bound (1.4) is always at least about as good and can be much better than the bound (1.3). Demmel and Veselić [13] showed that (1.4) also holds under a more general perturbation of the type

$$
\left|\delta H_{i j}\right| \leq \varepsilon\left(H_{i i} H_{j j}\right)^{1 / 2}
$$

and that the standard Jacobi method computes the eigenvalues with nearly this accuracy. Barlow and Demmel [2] considered scaled diagonally dominant matrices, i.e. matrices of the form

$$
H=D A D, \quad A=E+M,
$$

where $D$ is diagonal and non-singular, $E$ is diagonal with elements \pm 1 , $\operatorname{diag}(M)=0$, and $\|M\|_{2}=\zeta<1$. They showed that for such matrices (1.2) implies

$$
\frac{\left|\lambda_{i}-\lambda_{i}^{\prime}\right|}{\lambda_{i}} \leq \frac{\varepsilon n^{2}}{1-\zeta}
$$

and that a version of bisection without previous tridiagonalization computes the eigenvalues with nearly this accuracy.

Our perturbation bound for the non-singular but possibly indefinite matrix $H$ is the following: set

$$
|H|=D \widehat{A} D
$$

where $\mathbf{I} \cdot \mathbf{I}$ is the spectral absolute value $\left(\boldsymbol{|} H \mathbf{|}\right.$ is symmetric square root of $\left.H^{2}\right)$, and $D=(\operatorname{diag}(|H|))^{1 / 2}$. Then

$$
\frac{\left|\lambda_{i}-\lambda_{i}^{\prime}\right|}{\left|\lambda_{i}\right|} \leq \frac{\varepsilon n}{\lambda_{\min }(\widehat{A})}
$$

holds under the perturbations of types (1.2) and under

$$
\left|\delta H_{i j}\right| \leq \varepsilon D_{i i} D_{j j}
$$

This bound is actually derived in the more general setting of positive definite Hermitian matrix pairs. By (1.5) it is always true that $\kappa(\widehat{A}) \leq n \kappa(|H|)=n \kappa(H)$, and it 
is possible that $\kappa(\widehat{A}) \ll \kappa(H)$. Therefore, our bound (1.9) is always at least about as good and can be much better than the bound (1.3). If $H$ is positive definite, our bound reduces to the bound (1.4). If $H$ is scaled diagonally dominant, our bound is similar to the bound (1.7) (see Chap. 2).

Since the implicit $J$-orthogonal Jacobi method works on the pair $G, J$, we also need the perturbation theory in the case when $H$ is perturbed by its factors. Let $\lambda_{i}^{\prime}$ be the $i$-th eigenvalue of a perturbed matrix $(G+\delta G) J(G+\delta G)^{T}$. Set $G=B D$ where $D$ is diagonal positive definite, and columns of $B$ have unit norms. Set $\delta G=\delta B D$. If $\|\delta B\| \leq \varepsilon$ and $\varepsilon / \sigma_{\min }(B)<1$, where $\sigma_{\min }(B)$ is the smallest singular value of $B$, then

$$
\left(1-\varepsilon / \sigma_{\min }(B)\right)^{2} \leq \frac{\lambda_{i}^{\prime}}{\lambda_{i}} \leq\left(1+\varepsilon / \sigma_{\min }(B)\right)^{2} .
$$

Here $H$ needs not to be non-singular, but $G$ must have full column rank.

Error bounds for the eigenvalues computed by our algorithm follow from (1.9), (1.11), and the error analysis of our algorithm. Let $H$ be non-singular. Suppose that both steps of our algorithm are performed in a floating-point arithmetic with precision $\varepsilon$. Let $G, J$ be the output of the symmetric indefinite decomposition. Write $G=D_{G} B_{G}$, where $D_{G}$ is diagonal positive definite, and rows of $B_{G}$ have unit norms. For the matrices $G_{m}$ obtained by the implicit $J$-orthogonal Jacobi method write $G_{m}=B_{m} D_{m}$, where $D_{m}$ is diagonal and positive definite, and columns of $B_{m}$ have unit norms. Let $G_{M}, J$ be the last pair obtained by the implicit Jacobi, and let $G_{M}$ satisfy the stopping criterion,

$$
\left|\left(B_{M}^{T} B_{M}\right)_{i j}\right| \leq \text { tol }, \quad \text { for all } i \neq j .
$$

tol is a small constant, usually $n$ times machine precision. This relative stopping criterion is a natural consequence of (1.11) and it has been used before [13, 29, 31]. Let $\lambda_{i}^{\prime}$ be the $i$-th calculated eigenvalue. Then

$$
\frac{\left|\lambda_{i}-\lambda_{i}^{\prime}\right|}{\left|\lambda_{i}\right|} \leq \frac{272 n^{2} \varepsilon}{\lambda_{\min }\left(D_{G}^{-1}\left|G J G^{T}\right| D_{G}^{-1}\right)}+2 \varepsilon \sum_{m=0}^{M-1} \frac{C_{m}}{\sigma_{\min }\left(B_{m}\right)}+n \cdot t o l+n^{2} \varepsilon
$$

holds with the relative error of $O(\varepsilon)$. Here $G J G^{T}$ denotes the exact product of the calculated factors of $H$, and $C_{m}$ are moderate constants. Throughout the thesis the formulation "with the relative error of $O(\varepsilon)$ " means that $\varepsilon$ is replaced by $\varepsilon(1+K \varepsilon)$, where $0<K \ll 1 / \varepsilon$. The first quotient on the right hand side of (1.12) comes from (1.9) and the error analysis of the symmetric indefinite decomposition, and the rest comes from (1.11) and the error analysis of the implicit Jacobi. The bound (1.12) has the same order of magnitude as predicted by the perturbation theory of (1.9) and (1.11) if $\lambda_{\min }\left(D_{G}^{-1}\left|G J G^{T}\right| D_{G}^{-1}\right)$ is not much smaller than $\lambda_{\min }(\widehat{A})$ of $(1.9)$, and if the quantity $1 / \sigma_{\min }\left(B_{m}\right)$ does not grow much during the implicit Jacobi (note that in exact arithmetic $\lim _{m \rightarrow \infty} \sigma_{\min }\left(B_{m}\right)=1$ ). We have strong numerical evidence for both these facts, but our theoretical understanding is weaker. Moreover, we have observed that $1 / \sigma_{\min }\left(B_{0}\right)$ is usually very small. This means that: 
- the error induced by symmetric indefinite decomposition is usually larger than the error induced by implicit Jacobi,

- our method becomes even more accurate if the (almost) exact factor $G$ is readily supplied,

- our algorithm is usually faster than the standard Jacobi.

Similar observations were made by Demmel and Veselić [13] for the positive definite $H$. Moreover, since the theoretical results about the behaviour of $1 / \sigma_{\min }\left(B_{m}\right)$ are independent of the type of rotations used, we conclude that there is no reason to avoid hyperbolic rotations. Deichmöller [8] considered the solving of the generalized singular value problem with Jacobi-type methods, and obtained similar results about the growth of the condition of scaled matrices and a good error analysis for nonorthogonal rotations used there.

Our approach to the eigenvector perturbation theory is that of [20] which deals with the norm-estimates of the eigenprojections and thus allows the treatment of multiple and clustered eigenvalues. Our error bound holds, however, only for the eigenvectors corresponding to single eigenvalues. Let, as above, $H$ and $G$ both be non-singular. Let $v_{i}$ and $v_{i}^{\prime}$ be the eigenvectors of $\lambda_{i}$ and $\lambda_{i}^{\prime}$, respectively. Let $\lambda_{G, i}$ be the $i$-th eigenvalue of $G J G^{T}$. Then, less formally stated,

$$
\left\|v_{i}^{\prime}-v_{i}\right\|_{2} \leq \frac{\sqrt{2} \eta}{r g\left(\lambda_{i}\right)}+\frac{4 \sqrt{2} \bar{\eta}}{r g_{G}\left(\lambda_{G, i}\right)}+O\left(n^{2} \varepsilon\right)
$$

Here $\eta$ is the first quotient of the right hand side of (1.12), and $\bar{\eta}$ is approximately 1.5 times the rest of the right hand side of (1.12). $r g(\lambda)$ and $r g_{G}(\lambda)$ are two kinds of relative gaps between the eigenvalues, e.g. for $\lambda>0$ we set

$$
r g_{G}(\lambda)=\min \left\{1, \frac{\lambda_{R}-\lambda}{\lambda_{R}+\lambda}, \frac{\lambda-\lambda_{L}}{\lambda+\lambda_{L}}\right\}
$$

Here $\lambda_{L}$ and $\lambda_{R}$ are the left and right neighbours of $\lambda$ in the spectrum, and the quotients containing them are defined only if $\lambda_{L}, \lambda_{R}$ exist and are positive, respectively. This result applied to positive definite or scaled diagonally dominant $H$ is similar to the corresponding results of $[13,2]$, although with a different definition of relative gap. The bound (1.13) compares favourably to the standard eigenvector result [22] which, for the perturbation of the type (1.2), says

$$
\left\|v_{i}^{\prime}-v_{i}\right\| \leq \frac{n \varepsilon\|H\|_{2}}{\min _{i \neq j}\left|\lambda_{i}-\lambda_{j}\right|}+O\left(\varepsilon^{2}\right)
$$

In fact, if $H$ has two or more tiny eigenvalues, then the above minimum is necessarily small for some $i$ 's, but the relative gaps may be large. 
To illustrate our theory consider the matrix

$$
H=\left[\begin{array}{rrrr}
1600 & -300 & 14 & 300000 \\
-300 & 43.5 & -4.75 & -423212 \\
14 & -4.75 & 0.1875 & 19800 \\
300000 & -423212 & 19800 & 3207938 \cdot 10^{3}
\end{array}\right]
$$

whose all elements are sums of powers of 2 , and are exactly stored in IEEE single precision, $\varepsilon \approx 10^{-8}$. We have

$$
\frac{1}{\lambda_{\min }\left(D_{G}^{-1}\left|G J G^{T}\right| D_{G}^{-1}\right)} \approx 18, \quad \frac{1}{\sigma_{\min }(B)} \approx 1.1
$$

so we expect that the single precision version of our algorithm $\left(\varepsilon \approx 10^{-8}\right)$ returns six or seven correct decimal digits. The eigenvalues of $H$ are

$$
\begin{aligned}
& \lambda_{1}=-54.043364 \\
& \lambda_{2}=-0.0283096849 \\
& \lambda_{3}=1613.74866 \\
& \lambda_{4}=3207938084.0105
\end{aligned}
$$

Here the digits which are common to our algorithm and the LAPACK routine DSYEV which implements tridiagonalization followed by QR iteration (all performed in IEEE double precision, $\varepsilon \approx 10^{-16}$ ) are displayed. Our algorithm, QR algorithm from the LAPACK routine SSYEV, and the standard Jacobi, all in single precision, computed the following eigenvalues:

$$
\begin{array}{rrrr} 
& \text { OUR ALG. } & \text { SSYEV } & \text { JACOBI } \\
\lambda_{1} & -54.043369 & -55.990593 & -54.043369 \\
\lambda_{2} & -0.02830968 & -0.0326757 & -0.02830995 \\
\lambda_{3} & 1613.7487 & 1651.6652 & 1613.7486 \\
\lambda_{4} & 3207938000 & 3207938000 & 3207938000
\end{array}
$$

Therefore, our algorithm computed the eigenvalues with the predicted relative accuracy, QR has totally missed the absolutely smallest eigenvalue (and two more are very inaccurate), and the standard Jacobi computed the absolutely smallest eigenvalue somewhat less accurately than our algorithm. Note that $H$ is far from being scaled diagonally dominant which shows that our results are a non-trivial generalization of those of [2]. The algorithms behaved similarly on all such matrices for which the bound (1.12) is small and $\kappa(H)$ is large.

To explain the loss of accuracy in QR and the standard Jacobi algorithm note that both algorithms do all of their work on an indefinite matrix. Let $H_{m}$ be the sequence of matrices generated in floating-point arithmetic by either of those algorithms. Further, let $\widehat{A}_{m}$ be obtained from $H_{m}$ according to (1.8). In both algorithms it frequently 
happens that $\max _{m} \kappa\left(\widehat{A}_{m}\right) \gg \kappa(\widehat{A})$, which can, in turn, result in the loss of accuracy. In $\mathrm{QR}$ algorithm accuracy can be lost during the tridiagonalization, as well as during the iterative part. To illustrate the loss of accuracy during the tridiagonalization consider the matrix

$$
H=\left[\begin{array}{ccc}
10^{20} & 1 & 1 \\
1 & 1 & 1 \\
1 & 1 & 10^{20}
\end{array}\right]
$$

for which $\kappa(\widehat{A}) \approx 1$ and $\kappa(H) \approx 10^{20}$. The tridiagonalization, which consists of one Givens rotation, yields the matrix

$$
H_{1}=\left[\begin{array}{ccc}
10^{20} & \sqrt{2} & 0 \\
\sqrt{2} & 10^{20}+\frac{3}{2} & 10^{20}-\frac{1}{2} \\
0 & 10^{20}-\frac{1}{2} & 10^{20}-\frac{1}{2}
\end{array}\right]
$$

for which $\kappa\left(\widehat{A}_{1}\right) \approx \kappa(H)$. In floating-point arithmetic with precision $\varepsilon=10^{-16}$ the computed matrix $H_{1}$ is exactly singular indicating total loss of accuracy. Demmel [10] gives an example of a well-behaved tridiagonal matrix where $\kappa\left(\widehat{A}_{m}\right)$ almost reaches $\kappa(H)$ during QR iterations, which, in turn, results in the total loss of accuracy.

The main difference between indefinite non-singular and positive definite matrices is the following: for positive definite $H$ the perturbations of the types (1.2) and (1.6) are equivalent in the sense that if $H$ is insensitive to the one type, it is insensitive to the other type, and vice versa [13]. For indefinite $H$ this is not the case. Indeed, let

$$
H=\left[\begin{array}{lll}
1 & 1 & 1 \\
1 & 0 & 0 \\
1 & 0 & \epsilon
\end{array}\right]
$$

where $\epsilon$ is small (this matrix is considered in Sections 2.3, 4.3). $H$ is obviously very sensitive to perturbations of the type (1.10) so the bound (1.9) must necessarily be large. On the other side, $H$ is insensitive to small relative componentwise perturbations (1.2). This shows that we are still unable to completely characterize all well-behaved symmetric matrices. Due to large errors in the symmetric indefinite decomposition, our algorithm computes the eigenvalues with large relative errors. We can, however, easily obtain an almost exact factorization of $H$ (one way is to change the choice of pivots in the symmetric indefinite decomposition), and then the implicit Jacobi computes the eigensolution to nearly full working accuracy. This shows that we have not completely reached our ideal: if the matrix is well behaved, our algorithm should compute the eigenvalues with nearly this accuracy.

The thesis is organized as follows: Chapter 2 presents the new perturbation theory. This chapter, except Subsection 2.3.1, is due to Veselić and Slapničar [32]. The results of Veselić and Slapničar are included mainly for the sake of completeness. In Chapter 3 we first describe the $J$-orthogonal Jacobi method for the pair $H, J$, where $H$ is positive definite, and give its error analysis. Although this explicit method is rarely 
used, its error analysis is the basis for the later analysis of the implicit method. The error analysis consists of two steps. We first show that one step of $J$-orthogonal Jacobi method satisfies the perturbation bounds of Chapter 2. Then we combine one-step error analysis with the perturbation bounds to obtain overall error bounds for the eigensolution computed by $J$-orthogonal Jacobi method. In Subsection 3.2.2 we give known and new results concerning the upper bound for $1 / \sigma_{\min }\left(B_{m}\right)$. Then we describe and analyse the implicit $J$-orthogonal Jacobi method, and do the same for the implicit method with fast and fast self-scaling rotations. The latter are used to suppress possible underflow/overflow when accumulating the diagonal of the fast rotations. In Chapter 4 we define the symmetric indefinite decomposition (1.1) and give its error analysis. In Section 4.3 we combine the error analysis of the symmetric indefinite decomposition, error analysis of the implicit $J$-orthogonal Jacobi method, and the perturbation bounds of Chap. 2, to obtain the final error bounds for the computed eigensolution of the real symmetric eigenvalue problem. There we also shortly refer to the singular case, and state some open problems. In Section 4.4 we give an interesting theoretical result saying that the condition of the scaled matrix $G^{T} G$, $\kappa\left(B^{T} B\right)$, is bounded by a function of $n$ irrespective of the condition of the starting matrix $H$. In Chapter 5 we present results of our numerical experiments. Main tests were performed by comparing QR algorithms from LAPACK, standard Jacobi, and our algorithms in single and double precision. We also tested the behaviour of $\lambda_{\min }\left(D_{G}^{-1}\left|G J G^{T}\right| D_{G}^{-1}\right)$ and $\sigma_{\min }\left(B_{m}\right)$, and compared computation times. 


\section{Chapter 2}

\section{Floating-point perturbations of Hermitian matrices ${ }^{1}$}

\subsection{Introduction and preliminaries}

The standard perturbation result for the eigenvalue problem of a Hermitian matrix $H$ of order $n, H x=\lambda x$, reads [16]

$$
\left|\delta \lambda_{i}\right| \leq\|\delta H\|_{2}
$$

where

$$
\begin{array}{r}
\lambda_{1} \leq \lambda_{2} \leq \ldots \leq \lambda_{n} \\
\lambda_{1}^{\prime}=\lambda_{1}+\delta \lambda_{1} \leq \ldots \leq \lambda_{n}^{\prime}=\lambda_{n}+\delta \lambda_{n}
\end{array}
$$

are the eigenvalues of $H$ and $H+\delta H$, respectively. The perturbation matrix $\delta H$ is again Hermitian, and $\|\cdot\|_{2}$ is the spectral norm. The backward error analysis of various eigenvalue algorithms initiated by Wilkinson [33] follows the same pattern, i.e. the round-off error estimates are given in terms of norms. A more realistic perturbation theory starts from the fact that both the input entries of the matrix $H$ and the output eigenvalues are given in the floating-point form. Thus, a desirable estimate would read

$$
\max _{i}\left|\frac{\delta \lambda_{i}}{\lambda_{i}}\right| \leq C \max _{i, j}\left|\frac{\delta H_{i j}}{H_{i j}}\right|,
$$

where we define $0 / 0=0$. Colloquially, "floating-point" perturbations are those with $\left|\delta H_{i j}\right| \leq \varepsilon\left|H_{i j}\right|, \varepsilon$ small. Similarly, we call a matrix "well-behaved" if (2.1.2) holds with a "reasonable" $C$, i.e. if the small relative changes in the matrix elements cause small relative changes in the eigenvalues. For the floating-point perturbations (2.1.1)

\footnotetext{
${ }^{1}$ Sections 2.1, 2.2 and 2.3 of this chapter are due to Veselić and Slapničar [32]. Subsection 2.3.1 is new.
} 
implies (2.1.2) with $C=\sqrt{n} \cdot \kappa(H) \equiv \sqrt{n} \cdot\|H\|_{2}\left\|H^{-1}\right\|_{2}$, and this bound is almost attainable. This is illustrated by the positive definite matrix

$$
H=\left[\begin{array}{cc}
1 & 1 \\
1 & 1+\varepsilon
\end{array}\right], \quad 0<\varepsilon \ll 1 .
$$

The small eigenvalue of $H$ is very sensitive to small relative changes in the matrix elements.

Our results generalize the results obtained in [12, 2, 13]. Demmel and Veselić [13] showed that for a positive definite matrix $H$ (2.1.2) holds with

$$
C=\frac{n}{\lambda_{\min }(A)}
$$

where

$$
A=(\operatorname{diag}(H))^{-1 / 2} H(\operatorname{diag}(H))^{-1 / 2}
$$

is the standard scaled matrix. The condition of $A$ can be much smaller and is never much larger than that of $H$. Indeed, since $A_{i i}=1$ it follows

$$
\frac{1}{\lambda_{\min }(A)} \leq \kappa(A) \leq \frac{n}{\lambda_{\min }(A)}
$$

whereas (1.5) implies

$$
\kappa(A) \leq n \cdot \kappa(H) .
$$

Similar results hold for the singular value problem [13].

The aim of this paper is to extend the above result to general non-singular Hermitian matrices. The nature of the estimate (2.1.2) shows that the non-singularity is a natural condition to require. We show (Th. 2.2.3) that (2.1.2) holds for a non-singular Hermitian matrix $H$ with

$$
C=\||A|\|_{2}\left\|\widehat{A}^{-1}\right\|_{2}
$$

where

$$
H=D A D, \quad \widehat{A}=D^{-1}|H| D^{-1} .
$$

Here $D$ is any scaling matrix, i.e. a positive definite diagonal matrix, and $|\cdot|,|\cdot|$ denote the two kinds of absolute value functions, "pointwise" and "spectral":

$$
|A|_{i j}=\left|A_{i j}\right|, \quad \quad|H|=\sqrt{H^{2}},
$$

respectively. Note that $\|A\|_{2} \leq\||A|\|_{2} \leq \sqrt{n}\|A\|_{2}$ holds for any matrix $A$. The scaling $D$ is typically, but not necessarily of the standard form $D=(\operatorname{diag}|H|)^{1 / 2}$. This result is stated and proved in a more general setting, namely that of a matrix pair $H, K$ with $K$ positive definite, thus properly generalizing corresponding results of $[2,13]$. Our eigenvector result, stated in Subsect. 2.2.1, concerns the case of a single non-singular Hermitian matrix and it essentially generalizes the norm-estimates from $[2,13]$. An 
unpleasant point of our theory is that the matrix $|H|$, which has to be scaled, is not easy to compute. Moreover, the set of well-behaved indefinite Hermitian matrices is not scaling-invariant.

Barlow and Demmel [2] showed that for matrices of the type

$$
H=D(E+N) D
$$

where $D, E$ are diagonal, $E^{2}=I$, $\operatorname{diag}(N)=0$ and $\|N\|_{2}<1,(2.1 .2)$ holds with

$$
C=\frac{n}{1-\|N\|_{2}}
$$

The matrices (2.1.5) are called scaled diagonally dominant (s.d.d.). We show that for a s.d.d. matrix

$$
\||A|\|_{2}\left\|\widehat{A}^{-1}\right\|_{2} \leq n \frac{1+\||N|\|_{2}}{1-\|N\|_{2}} .
$$

Although this does not reproduce the constant $C$ in (2.1.6) (there is an extra factor $1+\||N|\|_{2} \leq 1+\sqrt{n}$ ), we see that s.d.d. matrices are included in our theory.

In the positive definite case the only well-behaved matrices are those which can be well scaled, i.e. for which the scaled matrix $A$ from (2.1.3) is "reasonably" conditioned. More precisely, if (2.1.2) holds for sufficiently small $\delta H$, then $\lambda_{\min }(A) \geq 2 /(1+C)$ for $A$ from (2.1.3). This, rather sharp result is proved in [32]. It improves a related result of [13] and also yields a slight improvement of the van der Sluis estimate (2.1.4).

In contrast to this, the choice of well-behaved indefinite matrices is, in a sense, richer. Writing

$$
H=G J G^{*}
$$

with $G^{*} G$ positive definite ( $G$ need not be square) and $J$ non-singular, the eigenvalue problem $H x=\lambda x$ converts into the problem

$$
\widehat{H} y=\lambda J^{-1} y, \quad \widehat{H}=G^{*} G .
$$

In Sect. 2.3 we prove the estimate of the type (2.1.2) for the problem (2.1.7) under the perturbations of the factor $\left|\delta G_{i j}\right| \leq \varepsilon\left|G_{i j}\right|$. The latter is a generalization of the singular value problem known as hyperbolic singular value problem [21]. The estimates again depend on the condition number of the matrix obtained by scaling $G^{*} G$. As an interesting application, we obtain floating-point perturbation estimates for matrices of the type

$$
H=\left[\begin{array}{cc}
H_{11} & H_{12} \\
H_{12}^{*} & 0
\end{array}\right],
$$

where $H_{12} H_{12}^{*}$ is positive definite. Note that this $H$ may be singular. As could be expected, the only well-behaved singular matrices are those where the rank defect can be read-off from the zero pattern.

Similarly as in [2], [13] we note the remarkable fact that our eigenvalue estimates are independent of the condition number of the corresponding eigenvector matrices 
- in generalized Hermitian eigenvalue problems they are not unitary and there is no upper bound for their condition. This phenomenon seems to be typical for the "floating-point" perturbation theory.

$æ$ 


\subsection{Well-conditioned scalings}

In this section we present perturbation results which are natural extensions of those from [2] and [13]. We first give a general perturbation result for the eigenvalues of the pair $H, K$ with $K$ positive definite. (An eigenvalue of the pair $H, K$ is a scalar $\lambda$ for which $\operatorname{det}(H-\lambda K)=0$.) For this purpose we introduce a new absolute value of $H$ relative to $K$ denoted by $|H|_{K}$. We then apply our general perturbation result to the floating-point perturbations of the matrices $H$ and $K$. Theorems 2.2.3 and 2.2.4 give two simplifications of the perturbation bounds and Th. 2.2.5 gives bounds for another, more general, type of perturbation where perturbing the zero elements is also allowed. Our theory applied to a single positive definite matrix slightly improves the corresponding results of [13]. It also improves the van der Sluis estimate (2.1.4) in some cases [32]. Then we apply our theory to a single non-singular indefinite matrix. We prove that our theory includes scaled diagonally dominant matrices [2]. We also characterize the class of matrices with the best perturbation bounds. At the end we give some examples, and also consider some singular matrices. In Subsect. 2.2.1 we consider the perturbation of the eigenvectors of a single non-singular matrix $H$.

Theorem 2.2.1 Let $H, K$ be Hermitian and $K$ positive definite. Set $K=Z Z^{*}$ and

$$
|H|_{K}=Z\left|Z^{-1} H Z^{-*}\right| Z^{*}
$$

$|H|_{K}$ is independent of the freedom of choice in $Z .^{2}$ Let $\delta H, \delta K$ be Hermitian perturbations such that for all $x \in \mathbf{C}^{n}$

$$
\left|x^{*} \delta H x\right| \leq \eta_{H} x^{*}|H|_{K} x, \quad\left|x^{*} \delta K x\right| \leq \eta_{K} x^{*} K x, \quad \eta_{H}, \eta_{K}<1
$$

holds. Let $\lambda_{i}$ and $\lambda_{i}^{\prime}$ be the increasingly ordered eigenvalues of the matrix pairs $H, K$ and $H^{\prime} \equiv H+\delta H, K^{\prime} \equiv K+\delta K$, respectively. Then $\lambda_{i}^{\prime}=0$ if and only if $\lambda_{i}=0$, and for non-vanishing $\lambda_{i}$ 's we have

$$
\frac{1-\eta_{H}}{1+\eta_{K}} \leq \frac{\lambda_{i}^{\prime}}{\lambda_{i}} \leq \frac{1+\eta_{H}}{1-\eta_{K}}
$$

Proof. Let $K=Z Z^{*}=F F^{*}$. Then $Z=F U$, where $U$ is a unitary matrix, and

$$
Z\left|Z^{-1} H Z^{-*}\right| Z^{*}=F U\left|U^{*} F^{-1} H F^{-*} U\right| U^{*} F^{*}=F\left|F^{-1} H F^{-*}\right| F^{*} .
$$

Thus, $|H|_{K}$ is independent of the freedom of choice in $Z$. From (2.2.2) it follows

$$
\begin{aligned}
x^{*}\left(H-\eta_{H}|H|_{K}\right) x & \leq x^{*}(H+\delta H) x \leq x^{*}\left(H+\eta_{H}|H|_{K}\right) x, \\
\left(1-\eta_{K}\right) x^{*} K x & \leq x^{*}(K+\delta K) x \leq\left(1+\eta_{K}\right) x^{*} K x .
\end{aligned}
$$

\footnotetext{
${ }^{2}$ For $H$ positive definite we obviously have $|H|_{K}=H$.
} 
Now note that the pair $H \pm \eta_{H}|H|_{K}$, $K$ has the same eigenvectors as the pair $H, K$ with the (again increasingly ordered) eigenvalues $\lambda_{i} \pm \eta_{H}\left|\lambda_{i}\right|$. Let $\widehat{\lambda}_{i}$ be the increasingly ordered eigenvalues of the pair $H^{\prime}, K$. The monotonicity property of the eigenvalues together with (2.2.4) yields immediately

$$
1-\eta_{H} \leq \frac{\widehat{\lambda}_{i}}{\lambda_{i}} \leq 1+\eta_{H} .
$$

It is also clear that $H$ and $H^{\prime}$ have the same inertia. ${ }^{3}$ The transition form $H^{\prime}, K$ to $H^{\prime}, K^{\prime}$ is similar. Note that both pairs have again the same inertia. If e.g. $\widehat{\lambda}_{i} \leq 0$, then $\lambda_{i}^{\prime} \leq 0$ and (2.2.5) implies

$$
\min _{S_{i}} \max _{x \in S_{i}} \frac{x^{*} H^{\prime} x}{\left(1-\eta_{K}\right) x^{*} K x} \leq \min _{S_{i}} \max _{x \in S_{i}} \frac{x^{*} H^{\prime} x}{x^{*} K^{\prime} x} \leq \min _{S_{i}} \max _{x \in S_{i}} \frac{x^{*} H^{\prime} x}{\left(1+\eta_{K}\right) x^{*} K x}
$$

where $S_{i}$ is any $i$-dimensional subspace of $\mathbf{C}^{n}$. In other words,

$$
\frac{\widehat{\lambda}_{i}}{1-\eta_{K}} \leq \lambda_{i}^{\prime} \leq \frac{\widehat{\lambda}_{i}}{1+\eta_{K}}
$$

Similarly, if $\widehat{\lambda}_{i} \geq 0$, then $\lambda_{i}^{\prime} \geq 0$, and we obtain

$$
\frac{\widehat{\lambda}_{i}}{1+\eta_{K}} \leq \lambda_{i}^{\prime} \leq \frac{\widehat{\lambda}_{i}}{1-\eta_{K}} .
$$

Now (2.2.7) and (2.2.8) combined with (2.2.6) give (2.2.3).

We now apply this result to the floating-point perturbations of matrix entries. Set

$$
\widetilde{C}(H, K)=\sup _{x \neq 0} \frac{|x|^{T}|H||x|}{x^{*} \mid H \mathbf{|}_{K} x}
$$

and

$$
\widetilde{C}(H)=\widetilde{C}(H, I)
$$

Obviously, $\widetilde{C}(H, K)$ is finite if and only if $H$ is non-singular. For every $H, K$ with $K$ positive definite, we have

$$
\widetilde{C}(H, K) \geq 1 .
$$

Indeed, if $\widetilde{C}(H, K)$ were less than one, then the matrices $H, K, \delta H=-H$ and $\delta K=0$ would satisfy the assumptions of Th. 2.2.1 and this would, in turn, imply that $H+\delta H$ is non-singular - a contradiction.

\footnotetext{
${ }^{3}$ In fact, $H$ and $H^{\prime}$ have the same null-spaces.
} 
Theorem 2.2.2 Let $H, K$ be Hermitian matrices with $H$ non-singular and $K$ positive definite. Let Hermitian perturbations $\delta H$ and $\delta K$ satisfy

$$
\left|\delta H_{i j}\right| \leq \varepsilon\left|H_{i j}\right|, \quad\left|\delta K_{i j}\right| \leq \varepsilon\left|K_{i j}\right|,
$$

such that

$$
\eta_{H}=\varepsilon \widetilde{C}(H, K)<1, \quad \eta_{K}=\varepsilon \widetilde{C}(K)<1 .
$$

Then the assumption (2.2.2) of Th. 2.2.1 is fulfilled, hence its assertion holds.

Proof. We have

$$
\left|x^{*} \delta H x\right| \leq|x|^{T}|\delta H||x| \leq \varepsilon|x|^{T}|H||x| \leq \varepsilon \widetilde{C}(H, K) x^{*}|H|_{K} x,
$$

and similarly

$$
\left|x^{*} \delta K x\right| \leq \varepsilon \widetilde{C}(K) x^{*} K x
$$

Q.E.D.

Th. 2.2.1 is a significant improvement over Lemma 1 and Th. 4 from [2] which require a more restrictive condition

$$
\left|x^{*} \delta H x\right| \leq \eta_{H}\left|x^{*} H x\right|
$$

which has non-trivial applications only for positive definite $H$.

The values $\widetilde{C}(H, K)$ and $\widetilde{C}(K)$ are not readily computable and we now exhibit a chain of simpler upper bounds for them.

Theorem 2.2.3 Let $H, K$ be as in Th. 2.2.2, and let $A, \widehat{A}$ and $B$ be defined by

$$
H=D A D, \quad|H|_{K}=D \widehat{A} D, \quad K=D_{1} B D_{1},
$$

where $D$ and $D_{1}$ are scaling matrices. Then

$$
\begin{array}{r}
\widetilde{C}(H, K) \leq\||A|\|_{2}\left\|\widehat{A}^{-1}\right\|_{2} \equiv C(A, \widehat{A}), \\
\widetilde{C}(K) \leq\||B|\|_{2}\left\|B^{-1}\right\|_{2} \equiv C(B),
\end{array}
$$

and $\eta_{H}=\varepsilon C(A, \widehat{A})<1, \eta_{K}=\varepsilon C(B)<1$ implies the assertion of Th. 2.2.1.

Proof. We have

$$
\begin{aligned}
|x|^{T}|H \| x| & =|x|^{T} D|A| D|x| \leq\||A|\|_{2} x^{*} D^{2} x \\
& \leq C(A, \widehat{A}) x^{*} D \widehat{A} D x=C(A, \widehat{A}) x^{*}|H|_{K} x
\end{aligned}
$$

and similarly

$$
|x|^{T}|K||x| \leq C(B) x^{*} D_{1} B D_{1} x=C(B) x^{*} K x .
$$


The constant $C(A, \widehat{A})$ cannot be uniformly improved. Indeed, take $H$ as diagonal with $H^{2}=I$ and let $H^{\prime}=H+\delta H$ be obtained by setting to zero any of the diagonal elements of $H$. Then the assertion of the above theorem, applied to the pair $H, K=I$ with $\delta K=0$, is obviously not true and we have $\eta_{H}=1, \eta_{K}=0$.

Of course, all this does not mean that Th. 2.2.3 covers all well behaved matrices. Next sections will show the contrary.

The constants $C(A, \widehat{A}), C(B)$ are further estimated as follows:

Theorem 2.2.4 Let $H, K$ be as in Th. 2.2.2, and let $A, \widehat{A}$ and $B$ be defined by (2.2.11), where $D, D_{1}$ are scalings. Then

$$
C(A, \widehat{A}) \leq \operatorname{Tr} \widehat{A}\left\|\widehat{A}^{-1}\right\|_{2}, \quad C(B) \leq \operatorname{Tr} B\left\|B^{-1}\right\|_{2},
$$

and $\eta_{H}=\varepsilon \operatorname{Tr} \widehat{A}\left\|\widehat{A}^{-1}\right\|_{2}<1, \eta_{K}=\varepsilon \operatorname{Tr} B\left\|B^{-1}\right\|_{2}<1$ implies the assertion of Th. 2.2.1.

Proof. Let

$$
Z^{-1} H Z^{-*}=U \Lambda U^{*}
$$

be an eigenvalue decomposition of $Z^{-1} H Z^{-*}$ with $U$ unitary and $\Lambda$ diagonal. Then $\left|Z^{-1} H Z^{-*}\right|=U|\Lambda| U^{*}$ and from (2.2.1) it follows

$$
|H|_{K}=Z U|\Lambda| U^{*} Z^{*}=G G^{*}
$$

where $G=Z U \sqrt{|\Lambda|}$. Furthermore,

$$
H=Z\left(Z^{-1} H Z^{-*}\right) Z^{*}=Z U \Lambda U^{*} Z^{*}=G J G^{*},
$$

where $J$ is diagonal with \pm 1 's on the diagonal. Setting $F=D^{-1} G$ for some positive definite diagonal $D$ and using the obvious estimate

$$
\left|\left(F J F^{*}\right)_{i j}\right| \leq \sqrt{\left(F F^{*}\right)_{i i}\left(F F^{*}\right)_{j j}},
$$

we obtain $\left|A_{i j}\right|^{2} \leq \widehat{A}_{i i} \widehat{A}_{j j}$, and hence $\||A|\|_{2} \leq \operatorname{Tr} \widehat{A}$. Similarly, $\||B|\|_{2} \leq \operatorname{Tr} B$, and the theorem now follows from the definitions of $C(A, \widehat{A})$ and $C(B)$.

Q.E.D.

For the standard scalings $D=\left(\operatorname{diag}|H|_{K}\right)^{1 / 2}, D_{1}=(\operatorname{diag} K)^{1 / 2}$, Th. 2.2.4 yields

$$
C(A, \widehat{A}) \leq n\left\|\widehat{A}^{-1}\right\|_{2}, \quad C(B) \leq n\left\|B^{-1}\right\|_{2} .
$$

In addition, the above upper bounds can accomodate another class of perturbations where perturbing the zero elements is also allowed. 
Theorem 2.2.5 Let $H, K$ be Hermitian matrices with $H$ non-singular and $K$ positive definite. Let Hermitian perturbations $\delta H$ and $\delta K$ satisfy

$$
\left|\delta H_{i j}\right| \leq \varepsilon D_{i i} D_{j j}, \quad\left|\delta K_{i j}\right| \leq \varepsilon D_{1, i i} D_{1, j j},
$$

such that

$$
\eta_{H}=\varepsilon n\left\|\widehat{A}^{-1}\right\|_{2}<1, \quad \eta_{K}=\varepsilon n\left\|B^{-1}\right\|_{2}<1 .
$$

Then the assumption (2.2.2) of Th. 2.2.1 is fulfilled, hence its assertion holds.

Proof. Let us define the matrix $E$ with $E_{i j}=1$. We have

$$
\left|x^{*} \delta H x\right| \leq|x|^{T}|\delta H||x| \leq \varepsilon|x|^{T} D E D|x| \leq \varepsilon\|E\|_{2} x^{*} D^{2} x \leq \varepsilon n\left\|\widehat{A}^{-1}\right\|_{2} x^{*}|H|_{K} x,
$$

and similarly

$$
\left|x^{*} \delta K x\right| \leq \varepsilon n\left\|B^{-1}\right\|_{2} x^{*} K x
$$

Q.E.D.

$æ$ 
Remark 2.2.6 Note that for the standard scaling the bounds of Theorems 2.2.3 and 2.2.5 differ by at most a factor $n$. Therefore, the relative error bounds which use $C(A, \widehat{A})$ and $C(B)$ actually allow both kinds of perturbations, (2.2.10) and (2.2.13), which makes them inappropriate in some cases (see Rem. 2.2.11 below).

When we apply our general theory to a single positive definite matrix $H(K=I)$, Th. 2.2.4 reproduces the main floating-point perturbation result of Th. 2.3 from [13], while Th. 2.2.2 is even sharper. The perturbations allowed by Th. 2.2.5 are of the form

$$
\left|\delta H_{i j}\right| \leq \varepsilon \sqrt{H_{i i} H_{j j}} .
$$

We now turn to the case of the single non-singular indefinite matrix $H$. We first prove that the class of matrices $H$ with well-behaved $C(A, \widehat{A})$ includes the already known class of scaled diagonally dominant matrices. We have

Theorem 2.2.7 Let

$$
H=D A D, \quad A=E+N,
$$

with $E=E^{*}=E^{-1}, E D=D E$, and $\|N\|_{2}<1$. If $\widehat{A}$ is defined by $|H|=D \widehat{A} D$, then

$$
C(A, \widehat{A}) \leq n \frac{1+\||N|\|_{2}}{1-\|N\|_{2}}
$$

Proof. Since $D$ commutes with $E$, there exists a unitary matrix $U$ which simultaneously diagonalizes $D$ and $E$, i.e.

$$
U^{*} D U=\Delta, \quad U^{*} E U=\operatorname{diag}( \pm 1)
$$

Since $\Delta$ is only a permuted version of the matrix $D$, there exists a permutation matrix $P$ such that $\Delta=P D P^{T}$. Setting $V=U P$, we have

$$
V^{*} D V=D, \quad V^{*} E V=E_{1},
$$

where $E_{1}$ is diagonal with \pm 1 's on the diagonal. Now perform the unitary transformation

$$
H_{1}=V^{*} H V=D\left(V^{*} E V+V^{*} N V\right) D=D\left(E_{1}+N_{1}\right) D .
$$

Here we used the fact that $D$ and $V$ commute. Also, $\left\|N_{1}\right\|_{2}=\|N\|_{2}$.

By Lemma 3 of [2] for any eigenpair $\lambda, y$ of $H_{1}$ we have

$$
\left(1-\left\|N_{1}\right\|_{2}\right)\|D y\|_{2}^{2} \leq|\lambda|\|y\|_{2}^{2} \leq\left(1+\left\|N_{1}\right\|_{2}\right)\|D y\|_{2}^{2} .
$$

Note that formally [2] needs that $N_{1}$ have a zero diagonal. It is easily seen that this condition is not necessary. For any eigenpair $\lambda, y$ of $H,(2.2 .16)$ implies

$$
\left(1-\|N\|_{2}\right)\|D y\|_{2}^{2} \leq|\lambda|\|y\|_{2}^{2} \leq\left(1+\|N\|_{2}\right)\|D y\|_{2}^{2} \text {. }
$$


Now let $H=Y \Lambda Y^{*}, Y^{*} Y=I, \Lambda=\operatorname{diag}\left(\lambda_{1}, \cdots, \lambda_{n}\right)$, be an eigenvalue decomposition of $H$. Then $|H|=Y|\Lambda| Y^{*}$ and

$$
\widehat{A}^{-1}=\left.D\left|H \mathbf{|}^{-1} D=D Y\right| \Lambda\right|^{-1 / 2}|\Lambda|^{-1 / 2} Y^{*} D
$$

Therefore,

$$
\left\|\widehat{A}^{-1}\right\|_{2}=\left\|D Y|\Lambda|^{-1 / 2}\right\|_{2}^{2} \leq n \max _{i}\left\|D y_{i}\right\|_{2}^{2} \frac{1}{\left|\lambda_{i}\right|} \leq \frac{n}{1-\|N\|_{2}}
$$

Here we have set $Y=\left[y_{1}, \cdots, y_{n}\right]$ and used (2.2.17) for every pair $\lambda_{i}, y_{i}$. The theorem now follows from ${ }^{4}$

$$
\||A|\|_{2} \leq\|I+|N|\|_{2} \leq 1+\||N|\|_{2} .
$$

Q.E.D.

The s.d.d. matrices are a special case of the matrices considered in Th. 2.2.7, that is, we do not require the diagonality of $E$. Note that the argument of [2] leading to the estimate (2.1.6) can be easily modified to hold under the conditions of Th. 2.2.7 as well.

Even though we could only bound our measure $C(A, \widehat{A})$ by $(2.2 .15)$ which is somewhat weaker than (2.1.6), we expect that $C(A, \widehat{A})$ is actually much better. The following example illustrates the power of our theory. Set

$$
\widehat{A}=\left[\begin{array}{ccc}
1 & 0.9 & 0.9 \\
0.9 & 1 & 0.9 \\
0.9 & 0.9 & 1
\end{array}\right], \quad \quad D=\left[\begin{array}{lll}
1 & & \\
& d & \\
& & d^{2}
\end{array}\right], \quad \quad d \geq 1
$$

Then $\left\|\widehat{A}^{-1}\right\|_{2}=10$. For $d=10^{2}$ the spectrum of $|H|=D \widehat{A} D$ is, properly rounded, $1.47 \cdot 10^{-1}, 1.90 \cdot 10^{3}, 1.00 \cdot 10^{8}$. Now $H$ is obtained from $|H|$ by just turning the smallest eigenvalue into its negative. We obtain

$$
H=\left[\begin{array}{ccc}
0.705 & 9.00 \cdot 10^{1} & 9.00 \cdot 10^{3} \\
9.00 \cdot 10^{1} & 1.00 \cdot 10^{4} & 9.00 \cdot 10^{5} \\
9.00 \cdot 10^{3} & 9.00 \cdot 10^{5} & 1.00 \cdot 10^{8}
\end{array}\right]
$$

with

$$
A=\left[\begin{array}{ccc}
0.705 & 0.9 & 0.9 \\
0.9 & 1 & 0.9 \\
0.9 & 0.9 & 1
\end{array}\right], \quad \quad\|A\| \leq 3
$$

Thus, $C(A, \widehat{A}) \leq 30$ and $H$ is far from being s.d.d.

\footnotetext{
${ }^{4}$ The case of the pair $H, K$ of s.d.d. matrices is not covered by this result (cf. a similar claim in $[2])$, although it seems highly probable that such a generalization holds.
} 
A natural question is to ask which matrix pairs or single non-singular matrices have the smallest $\eta_{H}, \eta_{K}$ in Th. 2.2.3. Obviously, $C(B) \geq 1$ and the equality is attained, if and only if $K$ is diagonal. In this case we can take $K=I$ and the whole problem reduces to the case of the single matrix $H$.

We first derive some useful inequalities. Set $x=K^{-1 / 2} y=D^{-1} z$. Then

$$
\left|x^{*} H x\right|=\left|y^{*} K^{-1 / 2} H K^{-1 / 2} y\right| \leq y^{*}\left|K^{-1 / 2} H K^{-1 / 2}\right| y=x^{*}|H|_{K} x,
$$

and thus

$$
\left|z^{*} A z\right| \leq z^{*} \widehat{A} z
$$

Similarly, $\left|x^{*} H^{-1} x\right| \leq x^{*}|H|_{K}^{-1} x$, and

$$
\left|z^{*} A^{-1} z\right| \leq z^{*} \widehat{A}^{-1} z
$$

Now we have $\left\|A^{-1}\right\|_{2} \leq\left\|\widehat{A}^{-1}\right\|_{2}$, and

$$
C(A, \widehat{A}) \geq\|A\|_{2}\left\|\widehat{A}^{-1}\right\|_{2} \geq\|A\|_{2}\left\|A^{-1}\right\|_{2} \geq 1 .
$$

Theorem 2.2.8 Let $H=D A D$ be Hermitian and non-singular and let $|H|=D \widehat{A} D$. Then

$$
C(A, \widehat{A})=\||A|\|_{2}\left\|\widehat{A}^{-1}\right\|_{2}=1
$$

if and only if $A$ is proportional to $P \operatorname{diag}\left(A_{1}, \cdots, A_{p}\right) P^{T}$, where each of the blocks $A_{i}$ has one of the forms

$$
1, \quad-1, \quad\left[\begin{array}{cc}
0 & e^{i \varphi} \\
e^{-i \varphi} & 0
\end{array}\right]
$$

$A$ and $D$ commute, and $P$ is a permutation matrix.

Proof. If $H$ has the form described above, then $|H|=D^{2}|A|=D^{2}$, i.e. $\widehat{A}=I$ and (2.2.22) holds.

Conversely, if (2.2.22) holds, then all inequalities in (2.2.21) go into equalities. Without loss of generality we can assume that

$$
\widehat{A}_{11}=1 \text {. }
$$

Now the equality $\|A\|_{2}\left\|A^{-1}\right\|_{2}=1$ means that

$$
A=c V, \quad c>0, \quad V=V^{-1}=V^{*} .
$$

From $|H|^{2}=H^{2}$ it follows that

$$
c^{2} V D^{2} V=\widehat{A} D^{2} \widehat{A} .
$$


This is equivalent to the unitarity of the matrix

$$
W=c D^{-1} \widehat{A}^{-1} V D .
$$

This, in turn, implies that $W$ is similar to $c \widehat{A}^{-1 / 2} V \widehat{A}^{-1 / 2}$. Since the latter matrix is also Hermitian, it must be unitary, i.e.

$$
c^{2} \widehat{A}^{-1 / 2} V \widehat{A}^{-1} V \widehat{A}^{-1 / 2}=I .
$$

This is equivalent to

$$
V\left(\frac{\widehat{A}}{c}\right)^{-1} V=\frac{\widehat{A}}{c} .
$$

We now use $\|A\|_{2}\left\|\widehat{A}^{-1}\right\|_{2}=\left\|(\widehat{A} / c)^{-1}\right\|_{2}=1$ which, together with (2.2.26) and (2.2.23), implies $\widehat{A}=I, c=1$. Now we can write $(2.2 .25)$ as $D^{2} A=A D^{2}$, i.e. $A$ and $D$ commute. Finally, we use $\||A|\|_{2}\left\|\widehat{A}^{-1}\right\|_{2}=\||A|\|_{2}=1$. By $c=1$, the relation $(2.2 .24)$ gives

$$
A=A^{-1}=A^{*} .
$$

Here we need the following

Lemma 2.2.9 Let $U^{*} U=I$ and $\||U|\|_{2}=1$. Then $|U|^{T}|U|=I$, i.e. each row of $U$ contains at most one non-vanishing element. If, in addition, $U$ is square, then $U$ is a (one sided) permutation of a diagonal matrix. Conversely, $|U|^{T}|U|=I$ implies $U^{*} U=I$ and $\||U|\|_{2}=1$.

Proof. From $U^{*} U=I$ it follows $\left(|U|^{T}|U|\right)_{i i} \equiv 1$. If $a_{i j}=\left(|U|^{T}|U|\right)_{i j} \neq 0$ for some pair $i \neq j$, then the submatrix

$$
\left[\begin{array}{cc}
1 & a_{i j} \\
a_{i j} & 1
\end{array}\right]
$$

of $|U|^{T}|U|$ has an eigenvalue greater than one - a contradiction to the assumption $\||U|\|_{2}=1$. The rest of the assertion is trivial.

Q.E.D.

To finish the proof of the theorem just use the lemma above and the hermiticity of $A$. Thus, up to a simultaneous permutation of rows and columns, $A$ is a direct sum of

$$
A_{i} \in\left\{1,-1,\left[\begin{array}{cc}
0 & e^{i \varphi} \\
e^{-i \varphi} & 0
\end{array}\right]\right\}, \quad i=1, \cdots, p .
$$

Q.E.D. 
The simple upper bounds in Th. 2.2.4 take their minimum $n$ on a much larger class of matrices, namely those with $A$ unitary and commuting with $D$. Indeed, from the proof of Th. 2.2.8 we immediately obtain

Corollary 2.2.10 Let $H, D, A$, and $\widehat{A}$ be as in Th. 2.2.8 such that $\widehat{A}_{11}=1$. Then the following assertions are equivalent:

(i) $\operatorname{Tr} \widehat{A}\left\|\widehat{A}^{-1}\right\|_{2}=n$,

(ii) $\widehat{A}=I$,

(iii) $A$ is unitary and commutes with $D$.

An example of such matrix is given by

$$
A=\left[\begin{array}{rrr}
c & s & 0 \\
s & -c & 0 \\
0 & 0 & 1
\end{array}\right], \quad D=\left[\begin{array}{lll}
d_{1} & & \\
& d_{1} & \\
& & d_{3}
\end{array}\right]
$$

where $s^{2}+c^{2}=1$ and $d_{1}, d_{3}>0$. Note that Th. 2.2 .7 concerns a certain sort of small perturbations of such matrices. Also note that the only positive definite matrices satisfying Cor. 2.2.10 are again diagonal ones.

The next natural question is: how good are the matrices $H=D A D$ with $A$ unitary, but not necessarily commuting with $D$ ? As an example take the matrix $H=D A D$ with

$$
A=\frac{1}{2}\left[\begin{array}{rrrr}
1 & -1 & -1 & -1 \\
-1 & 1 & -1 & -1 \\
-1 & -1 & 1 & -1 \\
-1 & -1 & -1 & 1
\end{array}\right], \quad D=\left[\begin{array}{llll}
d & & & \\
& 1 & & \\
& & 1 & \\
& & d
\end{array}\right]
$$

where $d>0$. Here $A$ is unitary, but it does not commute with $D$. The eigenvalues of $H$ are $\lambda_{1}=d^{2}, \lambda_{2}=d, \lambda_{3}=-d, \lambda_{4}=1$, and the corresponding eigenvectors are

$$
U=\left[\begin{array}{rrrr}
1 / \sqrt{2} & 1 / 2 & 1 / 2 & 0 \\
0 & -1 / 2 & 1 / 2 & 1 / \sqrt{2} \\
0 & -1 / 2 & 1 / 2 & -1 / \sqrt{2} \\
-1 / \sqrt{2} & 1 / 2 & 1 / 2 & 0
\end{array}\right]
$$

If we choose a relative perturbation of the form

$$
\delta H=\varepsilon d^{2} w w^{T}, \quad w=\left[\begin{array}{llll}
1 & 0 & 0 & 1
\end{array}\right]^{T},
$$

and set $H^{\prime}=H+\delta H$, we have $\left|\delta H_{i j}\right| \leq 2 \varepsilon\left|H_{i j}\right|$ and

$$
U^{T} H^{\prime} U=\operatorname{diag}\left(d^{2}, d,-d, 1\right)+\varepsilon d^{2} U^{T} w w^{T} U=\left[\begin{array}{cccc}
d^{2} & 0 & 0 & 0 \\
0 & d+\varepsilon d^{2} & \varepsilon d^{2} & 0 \\
0 & \varepsilon d^{2} & -d+\varepsilon d^{2} & 0 \\
0 & 0 & 0 & 1
\end{array}\right] .
$$


Therefore, $\lambda_{2}^{\prime}=d\left(\varepsilon d+\sqrt{1+\varepsilon^{2} d^{2}}\right)$ and $\left|\delta \lambda_{2}\right| /\left|\lambda_{2}\right|>\varepsilon d$, so $H$ is not well-behaved for large $d$. Since the matrix

$$
H A=\frac{1}{2}\left[\begin{array}{cccc}
d^{2}+d & 0 & 0 & -d^{2}+d \\
0 & d+1 & d-1 & 0 \\
0 & d-1 & d+1 & 0 \\
-d^{2}+d & 0 & 0 & d^{2}+d
\end{array}\right]
$$

is symmetric and positive definite, we conclude that $|H|=H A$. For $x=\left[\begin{array}{cccc}1 & 0 & 0 & 1\end{array}\right]^{T}$ we have

$$
\frac{|x|^{T}|H||x|}{x^{*}|H| x}=d
$$

and thus $\widetilde{C}(H) \rightarrow \infty$ as $d \rightarrow \infty$. This example shows that the properties of the matrix $A$ alone are in general not enough for the good behaviour of the indefinite matrix $H=D A D$. In other words, contrary to the positive definite case, an additional scaling $H_{1}=D_{1} H D_{1}$ of a well-behaved $H$ need not produce a well-behaved $H_{1}$.

Remark 2.2.11 Contrary to the positive definite case, for the indefinite matrices we do not have the result telling us that the matrix behaves well under the perturbations of the type (2.2.10) if and only if $\widetilde{C}(H)$ is small. Moreover, estimating $\widetilde{C}(H)$ with $C(A, \widehat{A})$ is in some cases not appropriate. For example, matrices of the type (2.1.8) behave well under the perturbations of the type (2.2.10) (see the following sections), but are very sensitive to the perturbations of the type (2.2.13) for the standard scaling. Therefore, $\eta_{H}$ from Th. 2.2.5 and then, in turn, $\eta_{H}$ from Th. 2.2.4 must neccessarily be large and some other kind of analysis is required.

Remark 2.2.12 (Some singular matrices). Although Th. 2.2.1 does not require the non-singularity of the unperturbed matrix $H$, the subsequent theory, as it stands, cannot handle singular matrices. However, for a single matrix of the type

$$
H=\left[\begin{array}{cc}
\widetilde{H} & 0 \\
0 & 0
\end{array}\right], \quad \widetilde{H} \text { non-singular },
$$

the condition $\left|\delta H_{i j}\right| \leq \varepsilon\left|H_{i j}\right|$ obviously preserves the zero structure and the problem trivially reduces to the perturbation of $\widetilde{H}$ to which our theory can be applied. For a pair $H, K$ with $H$ as above and $K$ positive definite of the form

$$
K=\left[\begin{array}{ll}
K_{11} & K_{12}^{*} \\
K_{12} & K_{22}
\end{array}\right]
$$

we proceed as follows: from the proof of Th. 2.2.2 we see that the perturbation on $K$ does not need the non-singularity of $H$. Furthermore, the non-zero eigenvalues of the pair $H, K$ coincide with the eigenvalues of the pair $\widetilde{H}, \widetilde{K}$, where $\widetilde{K}=K_{11}-$ 
$K_{12} K_{22}^{-1} K_{12}^{*}$. Thus, in perturbing $H$ the zero eigenvalues do not change and we can apply Th. 2.2.2 to the pair $\widetilde{H}, \widetilde{K}$. We obtain the full assertion of Th. 2.2.2 with $\widetilde{C}(\widetilde{H}, \widetilde{K})$ instead of $\widetilde{C}(H, K)$.

Similarly, Th. 2.2.3 holds where $A, \widehat{A}$ and $B$ are obtained by scaling $\widetilde{H},|\widetilde{H}|_{\widetilde{K}}$ and $K$, respectively. If, in addition, $H$ is positive semidefinite, then $\widetilde{\boldsymbol{H}} \widetilde{K}_{\widetilde{K}}=\widetilde{H}$, and Th. 2.2.3 and the subsequent theory hold with $A=\widehat{A}$ and $B$ obtained by scaling $\widetilde{H}$ and $K$, respectively.

It is readily seen that (2.2.28) is the only form (up to a permutation) of a positive semidefinite matrix whose eigenvalues behave well under the floating-point perturbations. As we shall see later, the indefinite case is more complicated in this aspect.

$æ$ 


\subsubsection{Perturbation of the eigenvectors}

In this subsection we consider the behaviour of the eigenvectors under the perturbations as in Th. 2.2.1. We consider the case of a single non-singular Hermitian matrix $H$ (i.e. $K=I, \delta K=0$ ). Like in $[2,13]$, this behaviour is influenced by a relative gap between the neighbouring eigenvalues. Our definition of relative gap is similar but not identical with the ones from $[2,13]$ which makes an exact comparison of (actually similar) results difficult. Our approach - in contrast to the one from $[2,13]$ - is that of [20] which deals with the norm-estimates of the spectral projections and thus allows the treatment of multiple and clustered eigenvalues. We also expect our bounds to be better than those of $[2,13]$, since they do not depend on $n$.

We now define the relative gap, $\operatorname{rg}(\lambda)$, for the possibly multiple eigenvalue $\lambda$ of $H$. To simplify the notation, as well as the statement and the proof of the following theorem, we shall assume that $\lambda$ is positive. Negative eigenvalues of $H$ are considered as the positive eigenvalues of the matrix $-H$. By $\lambda_{L}$ and $\lambda_{R}$ we denote the left and the right neighbour of $\lambda$ in the spectrum $\sigma(H)$ of $H$, respectively. We set

$$
r g(\lambda)= \begin{cases}\min \left\{\frac{\sqrt{\lambda}-\sqrt{\lambda_{L}}}{\sqrt{\lambda}}, \frac{\sqrt{\lambda_{R}}-\sqrt{\lambda}}{\sqrt{\lambda_{R}}}\right\} & \text { if } \lambda_{L}>0, \\ \min \left\{2(\sqrt{2}-1), \frac{\lambda_{R}-\lambda}{\lambda_{R}+\lambda}\right\} & \text { otherwise } .\end{cases}
$$

Theorem 2.2.13 Let $\lambda$ be a positive (possibly multiple) eigenvalue of a non-singular Hermitian matrix $H$, and let

$$
P=\frac{1}{2 \pi i} \int_{\Gamma} R_{\mu} d \mu, \quad R_{\mu}=(\mu I-H)^{-1},
$$

be the corresponding eigenprojection. Here $\Gamma$ is a curve around $\lambda$ which separates $\lambda$ from the rest of the spectrum. Let $P+\delta P$ be the corresponding spectral projection of the matrix $H+\delta H$ with $\left|x^{*} \delta H x\right| \leq \eta x^{*}|H| x$. Then

$$
\|\delta P\|_{2} \leq \begin{cases}\frac{\eta}{r g(\lambda)} \cdot \frac{1}{1-\left(1+\frac{1}{r g(\lambda)}\right) \eta} & \text { for } \lambda_{L}>0,2 \sqrt{\lambda}-\sqrt{\lambda_{L}}<\sqrt{\lambda_{R}} \\ \frac{\eta}{r g(\lambda)} \cdot \frac{1}{1-\frac{\eta}{r g(\lambda)}} & \text { otherwise },\end{cases}
$$

provided that the right hand side is positive.

Proof. By setting

$$
\Delta=|H|^{-1 / 2} \delta H|H|^{-1 / 2}, \quad z_{\mu}=R_{\mu}|H|^{1 / 2}, \quad w_{\mu}=|H|^{1 / 2} R_{\mu}|H|^{1 / 2},
$$


we obtain $\|\Delta\|_{2} \leq \eta$ and

$$
\delta P=\frac{1}{2 \pi i} \int_{\Gamma} z_{\mu} \Delta \sum_{k=0}^{\infty}\left(w_{\mu} \Delta\right)^{k} z_{\mu} d \mu .
$$

Choosing $\Gamma$ as a circle around $\lambda$ with the radius $r$, we obtain

$$
\|\delta P\|_{2} \leq r z^{2} \eta \frac{1}{1-w \eta}
$$

with

$$
\begin{aligned}
z^{2} & =\max _{\mu \in \Gamma}\left\|z_{\mu}\right\|_{2}^{2}=\max _{\mu \in \Gamma} \max _{\nu \in \sigma(H)} \frac{|\nu|}{|\mu-\nu|^{2}} \\
w & =\max _{\mu \in \Gamma}\left\|w_{\mu}\right\|_{2}=\max _{\mu \in \Gamma} \max _{\nu \in \sigma(H)} \frac{|\nu|}{|\mu-\nu|}
\end{aligned}
$$

provided that $\eta<1 / w$. We obviously have

$$
\begin{aligned}
z^{2} & =\max \left\{\frac{\left|\lambda_{L}\right|}{\left(\lambda-r-\lambda_{L}\right)^{2}}, \frac{\lambda}{r^{2}}, \frac{\lambda_{R}}{\left(\lambda_{R}-\lambda-r\right)^{2}}\right\} \\
w & =\max \left\{\frac{\left|\lambda_{L}\right|}{\lambda-r-\lambda_{L}}, \frac{\lambda}{r}, \frac{\lambda_{R}}{\lambda_{R}-\lambda-r}\right\} .
\end{aligned}
$$

We first consider the case $\lambda_{L}>0$. If $2 \sqrt{\lambda}-\sqrt{\lambda_{L}}<\sqrt{\lambda_{R}}$, then by setting

$$
r=\sqrt{\lambda}\left(\sqrt{\lambda}-\sqrt{\lambda_{L}}\right)
$$

we obtain

$$
z^{2}=\frac{1}{\left(\sqrt{\lambda}-\sqrt{\lambda_{L}}\right)^{2}}, \quad w \leq \frac{\sqrt{\lambda}}{\sqrt{\lambda}-\sqrt{\lambda_{L}}}+1 .
$$

Here we used our assumption and the fact that both rightmost terms in (2.2.32) are decreasing functions of $\lambda_{R}$. Therefore,

$$
\|\delta P\|_{2} \leq \frac{\sqrt{\lambda}}{\sqrt{\lambda}-\sqrt{\lambda_{L}}} \eta \frac{1}{1-\left(1+\frac{\sqrt{\lambda}}{\sqrt{\lambda}-\sqrt{\lambda_{L}}}\right) \eta},
$$

and (2.2.31) holds. Positivity of the right hand side of (2.2.31) justifies, in turn, our choice of the same $\Gamma$ in the definitions of $P$ and $P+\delta P$ as follows: perturbation theorem for the eigenvalues implies that $\lambda_{L}$ can increase to at most $\lambda_{L}(1+\eta), \lambda_{R}$ can decrease to at least $\lambda_{R}(1-\eta)$, and the eigenvalues of $H+\delta H$ which correspond to $\lambda$ remain in the interval $[\lambda(1-\eta), \lambda(1+\eta)]$. Positivity of the right hand side of (2.2.31) always implies $r g(\lambda)>\eta$. This, together with our choice of $r$, implies that 
$\Gamma$ contains no points of the spectrum of $H+\delta H$ and that the interior of $\Gamma$ contains exactly those eigenvalues of $H+\delta H$ which correspond to $\lambda$. This remark holds for the subsequent cases, as well.

If $2 \sqrt{\lambda}-\sqrt{\lambda_{L}} \geq \sqrt{\lambda_{R}}$, then by setting

$$
r=\sqrt{\lambda}\left(\sqrt{\lambda_{R}}-\sqrt{\lambda}\right)
$$

we obtain

$$
z^{2}=\frac{1}{\left(\sqrt{\lambda_{R}}-\sqrt{\lambda}\right)^{2}}, \quad w=\frac{\sqrt{\lambda_{R}}}{\sqrt{\lambda_{R}}-\sqrt{\lambda}} .
$$

Here we used our assumption and the fact that both leftmost terms in the right hand side of (2.2.32) are increasing functions of $\lambda_{L}>0$. Therefore,

$$
\|\delta P\|_{2} \leq \frac{\sqrt{\lambda}}{\sqrt{\lambda_{R}}-\sqrt{\lambda}} \eta \frac{1}{1-\frac{\sqrt{\lambda_{R}}}{\sqrt{\lambda_{R}}-\sqrt{\lambda}} \eta}
$$

and (2.2.31) holds. If $\lambda$ is the largest positive eigenvalue (i.e. $\lambda_{R}$ does not exist), then by setting $r$ as in (2.2.33) we obtain

$$
z^{2}=\frac{1}{\left(\sqrt{\lambda}-\sqrt{\lambda_{L}}\right)^{2}}, \quad w=\frac{\sqrt{\lambda}}{\sqrt{\lambda}-\sqrt{\lambda_{L}}}
$$

and (2.2.31) holds again.

If $\lambda_{L}<0$ or if $\lambda_{L}$ does not exist, we proceed as follows: if $\operatorname{rg}(\lambda)=2(\sqrt{2}-1)$ (if $\lambda_{R}$ exists, this implies $\left.\lambda(4 \sqrt{2}+5) \leq \lambda_{R}\right)$, then by setting

$$
r=2(\sqrt{2}-1) \lambda
$$

we obtain

$$
z^{2}=\frac{1}{4(\sqrt{2}-1)^{2} \lambda}, \quad w=\frac{1}{2(\sqrt{2}-1)},
$$

so (2.2.31) holds. Finally, if $r g(\lambda)=\left(\lambda_{R}-\lambda\right) /\left(\lambda_{R}+\lambda\right)$, then by setting

$$
r=\lambda \frac{\lambda_{R}-\lambda}{\lambda_{R}+\lambda}
$$

we obtain

$$
z^{2}=\frac{1}{\lambda}\left(\frac{\lambda_{R}+\lambda}{\lambda_{R}-\lambda}\right)^{2}, \quad w=\frac{\lambda_{R}+\lambda}{\lambda_{R}-\lambda}
$$

and (2.2.31) holds again.

Q.E.D.

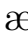




\subsection{Perturbations by factors}

In this section we consider perturbations of the eigenvalues of a single Hermitian matrix $H$ given in a factorized form

$$
H=G J G^{*},
$$

where $G$ need not to be square but must have full column rank, whereas $J$ is Hermitian and non-singular. A typical $J$ is

$$
J_{1}=\left[\begin{array}{cc}
I & 0 \\
0 & -I
\end{array}\right] \text {. }
$$

Here the unit blocks need not have the same dimension and one of them may be void. Such factorization is obtained e.g. by the symmetric indefinite decomposition of Chap. 4. We consider the changes of the eigenvalues and eigenvectors of $H$ under perturbation of $G$ while $J$ remains unchanged. Here it is natural to use the one-sided scaling $G=B D$.

For $J=I$ the problem reduces to considering singular values of $G$. We reproduce the result of [13] with somewhat better constants. The same technique allows an interesting floating-point estimate for the eigenvalues of $G$ (see [32]).

The section is organized as follows. Th. 2.3.1 gives a general perturbation theory, while Th. 2.3.2 applies this theory to the floating-point perturbations. In the following discussion we simplify the perturbation bounds analogously to the previous section. As an application we derive floating-point perturbation estimates for some classes of matrices not covered by Sect. 2.2.

Theorem 2.3.1 Let $H=G J G^{*}$ be as above and let $H^{\prime}=G^{\prime} J G^{*}$ with

$$
G^{\prime}=G+\delta G, \quad \quad\|\delta G x\|_{2} \leq \eta\|G x\|_{2},
$$

for all $x \in \mathbf{C}^{n}$ and some $\eta<1$. Then $H$ and $H^{\prime}$ have the same inertia and their non-vanishing eigenvalues $\lambda_{k}, \lambda_{k}^{\prime}$, respectively, satisfy the inequalities

$$
(1-\eta)^{2} \leq \frac{\lambda_{k}^{\prime}}{\lambda_{k}} \leq(1+\eta)^{2}
$$

Proof. We first show that the non-vanishing eigenvalues of $H$ coincide with the eigenvalues of the pair $G^{*} G, J^{-1}$. Indeed, since $G^{*} G$ is positive definite, there exists a non-singular $F$ such that

$$
F^{*} G^{*} G F=\Delta
$$

and

$$
F^{*} J^{-1} F=J_{1}
$$


are diagonal matrices, and $J_{1}$ is from (2.3.2). Then the eigenvalues of the pair $G^{*} G, J^{-1}$ are found on the diagonal of $\Delta J_{1}=J_{1} \Delta$. Set $U=G F \Delta^{-1 / 2}$. By (2.3.5) we have $U^{*} U=I$ (but not necessarily $U U^{*}=I$ ). Using (2.3.5) and (2.3.6) we obtain

$$
\begin{aligned}
H U & =G J G^{*} G F \Delta^{-1 / 2}=G J F^{-*} F^{*} G^{*} G F \Delta^{-1 / 2} \\
& =G J F^{-*} \Delta^{1 / 2}=G F F^{-1} J F^{-*} \Delta^{1 / 2} \\
& =G F\left(F^{*} J^{-1} F\right)^{-1} \Delta^{1 / 2}=U J_{1} \Delta
\end{aligned}
$$

Thus, the columns of $U$ are eigenvectors of $H$ and the eigenvalues of $H$ coincide with those of $G^{*} G, J^{-1}$. Furthermore, $U^{*} x=0$ implies $H x=0$, so the eigenvalues of $G^{*} G, J^{-1}$ are exactly all non-vanishing eigenvalues of $H$. By (2.3.3) we have

$$
(1-\eta)\|G x\|_{2} \leq\left\|G^{\prime} x\right\|_{2} \leq(1+\eta)\|G x\|_{2},
$$

so that everything said for $H$ holds for $H^{\prime}$ as well. In particular, $H$ and $H^{\prime}$ have the same inertia. Now square (2.3.7), use the monotonicity property from the proof of Th. 2.2.1 for the pairs $J^{-1}, G^{*} G$ and $J^{-1}, G^{*} G^{\prime}$, and take reciprocals in (2.2.7) and (2.2.8).

Q.E.D.

We now consider floating-point perturbations and scalings.

Theorem 2.3.2 Let $H=G J G^{*}$ be as in (2.3.1) and (2.3.2). Let $H^{\prime}=G^{\prime} J G^{*}$ where $G^{\prime}=G+\delta G$, and for all $i, j$ and some $\varepsilon>0$ holds

$$
\left|\delta G_{i j}\right| \leq \varepsilon\left|G_{i j}\right|
$$

Set

$$
\eta \equiv \frac{\varepsilon\||B|\|_{2}}{\sigma_{\min }(B)}
$$

where $B=G D^{-1}, D$ is diagonal and positive definite, and $\sigma_{\min }(B)$ is the smallest singular value of $B$. If $\eta<1$ then the assumptions of Th. 2.3.1 are fulfilled, hence its assertion holds.

Proof. For $x \in \mathbf{C}^{n}$ we have

$$
\begin{aligned}
\|\delta G x\|_{2} & \leq \varepsilon\||B| D|x|\|_{2} \leq \varepsilon\||B|\|_{2}\|D x\|_{2} \\
& \leq \frac{\varepsilon\||B|\|_{2}\|B D x\|_{2}}{\sigma_{\min }(B)}=\frac{\varepsilon\|B \mid\|_{2}\|G x\|_{2}}{\sigma_{\min }(B)} .
\end{aligned}
$$

Q.E.D.

By $\|\mid B\|_{2} \geq\|B\|_{2}$ we have

$$
\frac{\||B|\|_{2}}{\sigma_{\min }(B)} \geq \frac{\sigma_{\max }(B)}{\sigma_{\min }(B)} \geq 1 .
$$


Here both inequalities go over into equalities, if and only if $B$ has the property

$$
B^{*} B=\gamma^{2} I, \quad \gamma>0, \quad\|\mid B\|_{2}=\gamma,
$$

or, equivalently (Lemma 2.2.9), if and only if $|B|^{T}|B|=\gamma^{2} I$. Similarly as in Sect. 2.2 we can make a simplifying estimate

$$
\frac{\||B|\|_{2}}{\sigma_{\min }(B)} \leq \frac{\left(\operatorname{Tr}\left(B^{*} B\right)\right)^{1 / 2}}{\sigma_{\min }(B)},
$$

so that

$$
\eta=\frac{\varepsilon\left(\operatorname{Tr}\left(B^{*} B\right)\right)^{1 / 2}}{\sigma_{\min }(B)}<1
$$

again implies (2.3.3) and therefore (2.3.4). This yields a new "condition number"

$$
\frac{\left(\operatorname{Tr}\left(B^{*} B\right)\right)^{1 / 2}}{\sigma_{\min }(B)} \geq \sqrt{n},
$$

where the equality is attained if and only if $B^{*} B=\gamma^{2} I$. For the standard scaling where $\left(B^{*} B\right)_{i i}=1$ the relation $(2.3 .4)$ is implied by

$$
\eta=\frac{\varepsilon \sqrt{n}}{\sigma_{\min }(B)}<1 .
$$

This is a slight improvement over [13] for the case $J=I$ (our constant is $\sqrt{n}$ times better).

$æ$ 
For $J=I$ (or $J=-I$ ) we can handle the matrix $H=G G^{*}$ in two ways. If $G$ has full column rank, then we apply our theory as described in Theorems 2.3.1 and 2.3.2. If $G^{*}$ has full column rank, then we apply our theory to the matrix $\widehat{H}=G^{*} G$, whose non-vanishing eigenvalues are the eigenvalues of $H$. In the indefinite case $(J \neq \pm I)$ the situation is different. The following simple example illustrates this important asymmetry. Take

$$
G=[a, b], \quad \delta G=[\delta a, \delta b] .
$$

Our theory cannot be applied to

$$
H=G G^{*}=|a|^{2}+|b|^{2},
$$

but it works on

$$
H=G^{*} G
$$

where $G^{*}=\widetilde{B} \widetilde{D}, \widetilde{B}=\left[\begin{array}{ll}1 / \sqrt{2} & 1 / \sqrt{2}\end{array}\right]^{T}, \widetilde{D}=\left(|a|^{2}+|b|^{2}\right)^{1 / 2}$, thus giving $\eta=\varepsilon$ independently of $a$ and $b$. On the contrary, no theory can "save" the matrix

$$
H=G\left[\begin{array}{cc}
1 & 0 \\
0 & -1
\end{array}\right] G^{*}=|a|^{2}-|b|^{2}
$$

since

$$
\frac{|a+\delta a|^{2}-|b+\delta b|^{2}}{|a|^{2}-|b|^{2}}
$$

cannot be made small uniformly in $a, b$ if $|\delta a / a|$ and $|\delta b / b|$ are sufficiently small. ${ }^{5}$

Similarly as in Th. 2.2.5 we can show that a perturbation result holds under perturbations $\delta G$ defined by

$$
\left|\delta G_{i j}\right| \leq \varepsilon D_{j} \quad \text { for all } i, j
$$

where $D$ is a scaling. The above type of perturbation is less restrictive than (2.3.8), e.g. it allows us to change zero elements. We have

$$
\begin{aligned}
\|\delta G x\|_{2}^{2} & =\sum_{i, j, k} \bar{x}_{i} \delta \bar{G}_{j i} \delta G_{j k} x_{k} \leq n\left(\varepsilon \sum_{j}\left|D_{j} x_{j}\right|\right)^{2} \\
& \leq n^{2} \varepsilon^{2}\|D x\|_{2}^{2} \leq \frac{n^{2} \varepsilon^{2}\|G x\|_{2}^{2}}{\lambda_{\min }\left(B^{*} B\right)}
\end{aligned}
$$

hence (2.3.4) is implied by

$$
\eta=\frac{n \varepsilon}{\sigma_{\min }(B)}<1
$$

\footnotetext{
${ }^{5}$ In the indefinite case the values $\mu_{k}=\sqrt{\left|\lambda_{k}\right|} \operatorname{sign} \lambda_{k}$ are called the hyperbolic singular values [21].
} 
Similarly one shows that the estimate (2.3.4) is obtained under the perturbation

$$
\delta G=\delta B D, \quad \eta=\frac{\|\delta B\|_{2}}{\sigma_{\min }(B)}<1 .
$$

The following two examples show how Th. 2.3.2 can accomodate floating-point perturbations of some matrices which, in spite of Rem. 2.1, cannot be handled by the theory from Sect. 2.2. For the first example set

$$
H=\left[\begin{array}{cc}
A & F^{*} \\
F & 0
\end{array}\right]
$$

where $A$ is of order $m$ and $m \leq n-m$. Then $H=G J G^{*}$ with

$$
G=\left[\begin{array}{cc}
\frac{1}{2} A & I \\
F & 0
\end{array}\right], \quad J=\left[\begin{array}{cc}
0 & I \\
I & 0
\end{array}\right],
$$

where the unit blocks have the order $m$. Now the perturbation $\delta H$ of $H$ with $\left|H_{i j}\right| \leq$ $\varepsilon\left|H_{i j}\right|$ gives rise to a perturbation $\delta G$ of $G$ with $\left|\delta G_{i j}\right| \leq \varepsilon\left|G_{i j}\right|$, and Th. 2.3.2 holds e.g. with

$$
B=\left[\begin{array}{cc}
\frac{1}{2} A & I \\
F & 0
\end{array}\right]\left[\begin{array}{cc}
D^{-1} & 0 \\
0 & I
\end{array}\right]
$$

where $D$ is the standard scaling

$$
D_{i i}^{2}=\left(\frac{1}{4} A^{2}+F^{*} F\right)_{i i} .
$$

The requirement that $G$ have full column rank is equivalent to the same requirement on $F$. Note that this allows singular matrices $H$.

An even simpler case is the one with $A=0$. Then we can apply the theory to

$$
H=\left[\begin{array}{cc}
0 & F^{*} \\
F & 0
\end{array}\right]=\left[\begin{array}{cc}
0 & I \\
F & 0
\end{array}\right]\left[\begin{array}{cc}
0 & I \\
I & 0
\end{array}\right]\left[\begin{array}{cc}
0 & F^{*} \\
I & 0
\end{array}\right],
$$

as well as to

$$
H=\left[\begin{array}{cc}
0 & F \\
F^{*} & 0
\end{array}\right]=\left[\begin{array}{cc}
0 & F \\
I & 0
\end{array}\right]\left[\begin{array}{ll}
0 & I \\
I & 0
\end{array}\right]\left[\begin{array}{cc}
0 & I \\
F^{*} & 0
\end{array}\right]
$$

In any case, the non-vanishing eigenvalues of $H$ coincide with the singular values of $F$ taken with both signs. Now $\left|\delta G_{i j}\right| \leq \varepsilon\left|G_{i j}\right|$ means $\left|\delta F_{i j}\right| \leq \varepsilon\left|F_{i j}\right|$ and we can apply our theory in two ways:

(i) take e.g. (2.3.14) and use Th. 2.3.2 to obtain (2.3.4) with

$$
\eta=\frac{\||B|\|_{2}}{\sigma_{\min }(B)},
$$


where $B=F D^{-1},\left(B^{*} B\right)_{i i}=1$, or

(ii) apply Th. 2.3.2 to the factorized matrix $F F^{*}$ (with the same $B$ ) which yields a slightly better estimate

$$
(1-\eta)^{2} \leq \frac{\lambda_{k}^{\prime 2}}{\lambda_{k}^{2}} \leq(1+\eta)^{2} .
$$

In both cases the theory from Sect. 2 would require both $B B^{*}$ and $B^{*} B$ to scale well, which is certainly a further unnecessary restriction.

As a second example set

$$
H=\left[\begin{array}{ccc}
a & b & c \\
b & 0 & 0 \\
c & 0 & \alpha^{2}
\end{array}\right]
$$

We can e.g. decompose $H$ as

$$
H=\left[\begin{array}{ccc}
a / 2 & 1 & 0 \\
b & 0 & 0 \\
c & 0 & \alpha
\end{array}\right]\left[\begin{array}{lll}
0 & 1 & 0 \\
1 & 0 & 0 \\
0 & 0 & 1
\end{array}\right]\left[\begin{array}{ccc}
a / 2 & b & c \\
1 & 0 & 0 \\
0 & 0 & \alpha
\end{array}\right] .
$$

Now $\left|\delta H_{i j}\right| \leq \varepsilon\left|H_{i j}\right|$ again implies $\left|\delta G_{i j}\right| \leq \varepsilon\left|G_{i j}\right|$ and we can apply our theory as in the previous example. For e.g. $a=b=c=1$ we obtain $\||B|\|_{2}\left\|B^{-1}\right\|_{2}=$ $2+\sqrt{3}$, independently of $\alpha$. Especially, if $\alpha$ is small then even the absolutely smallest eigenvalue $\alpha^{2} / 2+O\left(\alpha^{4}\right)$ is well defined by the matrix elements of $H$. On the other side, the theory from Sect. 2 applied to $H, I$ gives nothing useful here. Indeed, as $\alpha \rightarrow 0$ we have

$$
|H|=\frac{1}{3}\left[\begin{array}{lll}
5 & 1 & 1 \\
1 & 2 & 2 \\
1 & 2 & 2
\end{array}\right]+O\left(\alpha^{2}\right)
$$

so that $C(A, \widehat{A})=O\left(1 / \alpha^{2}\right)$. Moreover, numerical experiments show that $\widetilde{C}(H)>$ $1 /|\alpha|$. Another very interesting approach to matrices of the above type is given by Demmel and Gragg [11].

$æ$ 


\subsubsection{Perturbation of the eigenvectors}

In this subsection we give the perturbation bounds for the eigenvectors of the nonsingular Hermitian matrix

$$
H=G J G^{*},
$$

under the perturbations as in Th. 2.3.1, i.e.

$$
\|\delta G x\|_{2} \leq \eta\|G x\|_{2}
$$

for every $x$.

As in $[2,13]$ and Subsect. 2.2.1, the behaviour of the eigenvectors is influenced by a relative gap between the neighbouring eigenvalues. Our definition of relative gap is similar but not identical with the one from [2, 13] and Subsect. 2.2.1, and our approach is again that of [20].

We now define the relative $g a p, r g_{G}(\lambda)$, and the eigenprojection $P$ for the possibly multiple eigenvalue $\lambda$ of $H$. To simplify the notation, as well as the statement and the proof of the following theorem, we shall assume that $\lambda$ is positive. Negative eigenvalues of $H$ are considered as the positive eigenvalues of the matrix $-H$. By $\lambda_{L}$ and $\lambda_{R}$ we denote the left and the right neighbour of $\lambda$ in the spectrum $\sigma(H)$ of $H$, respectively. We set

$$
\begin{aligned}
r g_{G}(\lambda) & =\min \left\{1, \frac{\lambda_{R}-\lambda}{\lambda_{R}+\lambda}, \frac{\lambda-\lambda_{L}}{\lambda+\lambda_{L}}\right\}, \\
P & =\frac{1}{2 \pi i} \int_{\Gamma} R_{\mu} d \mu, \quad R_{\mu}=(\mu I-H)^{-1},
\end{aligned}
$$

where $\Gamma$ is a curve around $\lambda$ which separates $\lambda$ from the rest of the spectrum of $H$. Here, as well as throughout the section, the terms containing $\lambda_{L}, \lambda_{R}$ are defined if $\lambda_{L}, \lambda_{R}$ exist and are positive, respectively.

Theorem 2.3.3 Let $\lambda$ be a positive (possibly multiple) eigenvalue of a non-singular Hermitian matrix $H=G J G^{*}$, and let $P$ be the corresponding eigenprojection. Let $P^{\prime}$ be the corresponding spectral projection of the matrix $H^{\prime}=G^{\prime} J\left(G^{\prime}\right)^{*}$, where $G^{\prime}=$ $G+\delta G$ and $\|\delta G x\|_{2} \leq \eta\|G x\|_{2}$ for every $x$.

Then

$$
\left\|P^{\prime}-P\right\|_{2} \leq \frac{4 \bar{\eta}}{r g_{G}(\lambda)} \cdot \frac{1}{1-\frac{3 \bar{\eta}}{r g_{G}(\lambda)}},
$$

where

$$
\bar{\eta}=\eta(2+\eta),
$$

provided that the right hand side in (2.3.2) is positive. 
Proof. Since $H$ and $H^{-1}$ have the same eigenvectors, we can define $P$ as

$$
P=\frac{1}{2 \pi i} \int_{\Gamma} S_{\mu} d \mu, \quad S_{\mu}=\left(\mu I-H^{-1}\right)^{-1}
$$

where $\Gamma$ is now a curve around $1 / \lambda$ which separates $1 / \lambda$ from the rest of the spectrum of $H^{-1}$. Therefore,

$$
P^{\prime}-P=\frac{1}{2 \pi i} \int_{\Gamma}\left(S_{\mu}^{\prime}-S_{\mu}\right) d \mu
$$

where

$$
S_{\mu}^{\prime}=\left(\mu I-H^{\prime-1}\right)^{-1}
$$

We can write

$$
S_{\mu}=\left(\mu I-G^{-*} J G^{-1}\right)^{-1}=G\left(\mu G^{*} G-J\right)^{-1} G^{*} \equiv G T_{\mu} G^{*}
$$

and analogously

$$
S_{\mu}^{\prime}=G^{\prime} T_{\mu}^{\prime}\left(G^{\prime}\right)^{*}, \quad T_{\mu}^{\prime}=\left(\mu\left(G^{\prime}\right)^{*} G^{\prime}-J\right)^{-1} .
$$

Now

$$
S_{\mu}^{\prime}-S_{\mu}=G\left(T_{\mu}^{\prime}-T_{\mu}\right) G^{*}+\Phi
$$

where

$$
\Phi=\delta G T_{\mu}^{\prime} G^{*}+G T_{\mu}^{\prime} \delta G^{*}+\delta G T_{\mu}^{\prime} \delta G^{*}
$$

Further,

$$
G\left(T_{\mu}^{\prime}-T_{\mu}\right) G^{*}=G T_{\mu}\left(T_{\mu}^{-1}-\left(T_{\mu}^{\prime}\right)^{-1}\right) T_{\mu}^{\prime} G^{*}=G T_{\mu} \mu \gamma T_{\mu}^{\prime} G^{*}
$$

where

$$
\gamma=-\delta G^{*} G-G^{*} \delta G-\delta G^{*} \delta G
$$

Inserting

$$
\gamma=G^{*} \Delta G
$$

and (2.3.4) into (2.3.7), we obtain

$$
G\left(T_{\mu}^{\prime}-T_{\mu}\right) G^{*}=S_{\mu} \mu \Delta G T_{\mu}^{\prime} G^{*}
$$

Using (2.3.4) and (2.3.8), we obtain

$$
\begin{aligned}
G T_{\mu}^{\prime} G^{*} & =G\left(T_{\mu}^{-1}-\mu G^{*} \Delta G\right)^{-1} G^{*} \\
& =G\left(I-\mu T_{\mu} G^{*} \Delta G\right)^{-1} T_{\mu} G^{*} \\
& =G\left(\left(T_{\mu} G^{*}\right)^{-1}-\mu \Delta G\right)^{-1} \\
& =S_{\mu}\left(I-\mu \Delta S_{\mu}\right)^{-1} .
\end{aligned}
$$


Inserting (2.3.10), (2.3.9), (2.3.6) and (2.3.5) into (2.3.3), we obtain

$$
\begin{aligned}
P^{\prime}-P= & \frac{1}{2 \pi i} \int_{\Gamma}\left[\mu S_{\mu} \Delta S_{\mu}\left(I-\mu \Delta S_{\mu}\right)^{-1}\right. \\
& +\delta G G^{-1} S_{\mu}\left(I-\mu \Delta S_{\mu}\right)^{-1}+S_{\mu}\left(I-\mu \Delta S_{\mu}\right)^{-1} G^{-*} \delta G^{*} \\
& \left.+\delta G G^{-1} S_{\mu}\left(I-\mu \Delta S_{\mu}\right)^{-1} G^{-*} \delta G^{*}\right] d \mu .
\end{aligned}
$$

Our assumption on $\delta G$ and the definition of $\Delta$ in (2.3.8) imply

$$
\begin{aligned}
\left\|\delta G G^{-1}\right\|_{2} & \leq \eta \\
\|\Delta\|_{2} & \leq 2\left\|\delta G G^{-1}\right\|_{2}+\left\|\delta G G^{-1}\right\|_{2}^{2} \leq \bar{\eta} .
\end{aligned}
$$

Choosing $\Gamma$ as a circle around $1 / \lambda$ with radius $r$, taking norms in (2.3.11), and using the above relations, we obtain

$$
\left\|P^{\prime}-P\right\|_{2} \leq r z(w+1) \bar{\eta} \frac{1}{1-\bar{\eta} w}
$$

where

$$
\begin{aligned}
w & =\max _{\mu \in \Gamma}\left\|\mu S_{\mu}\right\|_{2}=\max _{\mu \in \Gamma} \max _{\nu \in \sigma\left(H^{-1}\right)} \frac{|\mu|}{|\mu-\nu|}, \\
z & =\max _{\mu \in \Gamma}\left\|S_{\mu}\right\|_{2}=\max _{\mu \in \Gamma} \max _{\nu \in \sigma\left(H^{-1}\right)} \frac{1}{|\mu-\nu|} .
\end{aligned}
$$

Since $\Gamma$ is a circle, the maxima in the above relations are attained for $\mu$ 's which lie on the real axis.

If $\lambda_{R}$ exists, then we choose $r$ as

$$
r=\frac{1}{2} \min \left\{\frac{1}{\lambda}-\frac{1}{\lambda_{R}}, \frac{1}{\lambda_{L}}-\frac{1}{\lambda}\right\},
$$

and if $\lambda_{R}$ does not exist, then we choose $r$ as

$$
r=\frac{1}{2} \min \left\{\frac{1}{\lambda}, \frac{1}{\lambda_{L}}-\frac{1}{\lambda}\right\} .
$$

It is easy to see that we always have

$$
z=\frac{1}{r}
$$

Since $\mu=1 / \lambda \pm r$, we have

$$
w=\max \left\{\frac{1 / \lambda-r}{1 / \lambda-r-1 / \lambda_{R}}, \frac{1 / \lambda+r}{r}, \frac{1 / \lambda+r}{1 / \lambda_{L}-1 / \lambda-r}\right\} .
$$


Now if $r=\left(1 / \lambda-1 / \lambda_{R}\right) / 2$, then

$$
w=1+\frac{2}{\frac{\lambda_{R}-\lambda}{\lambda_{R}}} \leq 1+\frac{2}{r g_{G}(\lambda)} \leq \frac{3}{r g_{G}(\lambda)},
$$

and (2.3.2) follows by inserting this and $z=1 / r$ into (2.3.12).

If $r=\left(1 / \lambda_{L}-1 / \lambda\right) / 2$, then

$$
w=\frac{\lambda-\lambda_{L}}{\lambda+\lambda_{L}} \leq \frac{1}{r g_{G}(\lambda)}
$$

and (2.3.2) follows by inserting this and $z=1 / r$ into (2.3.12).

Finally, if $r=1 /(2 \lambda)\left(\lambda_{R}\right.$ does not exist), then $w=3$ and (2.3.2) follows by inserting this and $z=1 / r$ into (2.3.12).

Positivity of the right hand side of (2.3.2) justifies, in turn, our choice of the same $\Gamma$ in the definitions of $P$ and $P^{\prime}$ in (2.3.3) as follows: perturbation theorem for the eigenvalues implies that $1 / \lambda_{R}$ can increase to at most $1 /\left(\lambda_{R}(1-\eta)^{2}\right), 1 / \lambda_{L}$ can decrease to at least $1 /\left(\lambda_{L}(1+\eta)^{2}\right)$ and the eigenvalues of $H^{\prime-1}$ which correspond to $1 / \lambda$ remain in the interval $\left[1 /\left(\lambda(1+\eta)^{2}\right), 1 /\left(\lambda(1-\eta)^{2}\right)\right]$. Positivity of the right hand side of (2.3.2) always implies $r g_{G}(\lambda)>6 \eta$. This, together with our choice of $r$, implies that $\Gamma$ contains no points of the spectrum of $H^{\prime-1}$ and that the interior of $\Gamma$ contains exactly those eigenvalues of $H^{\prime-1}$ which correspond to $1 / \lambda$.

Q.E.D.

Remark 2.3.4 It is possible to prove theorem similar to Th. 2.3.3 for a cluster of eigenvalues, as well. All eigenvalues of the cluster must be either positive or negative. The relative gap for the cluster is then defined using $\lambda_{L}\left(\lambda_{R}\right)$ and the leftmost (rightmost) member of the cluster, respectively. The $r \cdot z$ term of (2.3.12) is then larger than 1, and smaller than the inverse of the relative gap of the cluster.

Note that we can in some cases actually prove better bounds than (2.3.2), but the differences are small, so we have decided to state and to prove the simpler version. Th. 2.3.3 is a generalization of the corresponding results from [13] since it allows $J \neq I$ and multiple eigenvalues.

Now suppose that $\lambda$ and $\lambda^{\prime}$ are both simple. Let $v$ and $v^{\prime}=v+\delta v$ be the corresponding unit eigenvectors, and let $\phi$ be the angle between them. Then $P=v v^{*}$, $P^{\prime}=v^{\prime}\left(v^{\prime}\right)^{*}$, and $P^{\prime}-P$ is a matrix of rank 2 with the non-trivial eigenvalues, say, $\gamma_{1}$ and $\gamma_{2}$. Since $\operatorname{Tr}\left(P^{\prime}-P\right)=0$, we have $\left|\gamma_{1}\right|=\left|\gamma_{2}\right| \equiv \gamma$. Now

$$
2 \gamma^{2}=\operatorname{Tr}\left[\left(P^{\prime}-P\right)\left(P^{\prime}-P\right)\right]=2 \sin ^{2} \phi,
$$

so that

$$
\left\|P^{\prime}-P\right\|_{2}=|\sin \phi| .
$$


This finally implies

$$
\|\delta v\|_{2}=2|\sin (\phi / 2)| \leq \sqrt{2}\left\|P^{\prime}-P\right\|_{2} .
$$

Combining the above relation with Th. 2.3 .3 we obtain the bound on $\|\delta v\|_{2}$. We expect this bound to compare favourably to the corresponding bounds from $[2,13]$ since it does not contain the factors $(n-1)$ or $(n-1)^{1 / 2}$, respectively.

$æ$ 


\section{Chapter 3}

\section{Error analysis of the $J$-orthogonal Jacobi methods}

\subsection{J-orthogonal Jacobi method}

The $J$-orthogonal Jacobi method solves the problem

$$
H x=\lambda J x, \quad x \neq 0,
$$

where $H=\left(H_{i j}\right)$ is a positive definite matrix,

$$
J=I_{\text {npos }} \oplus\left(-I_{n-n p o s}\right),
$$

npos is the number of the positive, and $n-n p o s$ is the number of the negative eigenvalues of the pair $H, J$. The algorithm, including the convergence theory, was proposed by Veselić [29]. For the sake of completeness we give the algorithm of the method and state the known convergence results.

In Chap. 2, we showed that there exists a nonsingular matrix $V$ which simultaneously diagonalizes $H$ and $J$ in the manner that

$$
V^{T} H V=D, \quad V^{T} J V=J,
$$

where $D=\left(D_{i}\right)$ is a positive definite diagonal matrix. The eigenvalues of the pair $H, J$ are the values $D_{i} \cdot J_{i}$ and the eigenvectors are the corresponding columns of $V$. The matrices for which $V^{T} J V=J$ are called $J$-orthogonal and they form a multiplicative group. (For a fixed $J$, of course.)

The $J$-orthogonal Jacobi method consists of an iterative application of the congruence transformation

$$
H^{\prime}=C^{T} H C
$$

where $C$ is the $J$-orthogonal plane rotation. From now on let $\widehat{A}$ denote the $2 \times 2$ pivot submatrix of the square matrix $A$. The matrix $C$ is defined as

$$
\widehat{C}=\left[\begin{array}{ll}
c_{i i} & c_{i j} \\
c_{j i} & c_{j j}
\end{array}\right],
$$


and the non-displayed elements are those of the identity matrix. The pair $(i, j)$ is the pivot pair. The $J$-orthogonality of the matrix $C$ implies that

$$
\left[\begin{array}{cc}
c_{i i} & c_{i j} \\
c_{j i} & c_{j j}
\end{array}\right]=\left\{\begin{array}{lc}
{\left[\begin{array}{cc}
c h & s h \\
s h & c h
\end{array}\right],} & \text { for } 1 \leq i \leq n \text { pos }<j \leq n, \\
{\left[\begin{array}{cc}
c s & s n \\
-s n & c s
\end{array}\right],} & \text { otherwise } .
\end{array}\right.
$$

Here $c h=\cosh y, s h=\sinh y, c s=\cos x$ and $s n=\sin x$ for some $y$ and $x$, respectively. These two types of rotations are called the hyperbolic and the trigonometric rotation, respectively. The parametar $x$ or $y$ is chosen so that the $i, j$-element of the transformed matrix is annihilated. Let

$$
\widehat{H}=\left[\begin{array}{ll}
a & c \\
c & b
\end{array}\right]
$$

Then

$$
\tan 2 x=\frac{2 c}{b-a}, \quad-\frac{\pi}{4} \leq x \leq \frac{\pi}{4},
$$

or

$$
\tanh 2 y=-\frac{2 c}{a+b} .
$$

We obtain the following algorithm (in the notation of [13]):

$æ$ 
Algorithm 3.1.1 Two-sided J-orthogonal Jacobi method for the problem (3.1.1). tol is a user defined stopping criterion. The matrix $V$ whose columns return the computed eigenvectors initially contains the identity.

repeat

for all pairs $i<j$

${ }^{*}$ compute the parameter hyp: hyp $=1$ for the hyperbolic and

hyp $=-1$ for the trigonometric rotation, respectively */

if $1 \leq i \leq n$ pos $<j \leq n$ then

hyp $=1$

else

hyp $=-1$

endif

${ }^{*}$ compute the $J$-orthogonal Jacobi rotation which diagonalizes

$\left[\begin{array}{ll}H_{i i} & H_{i j} \\ H_{j i} & H_{j j}\end{array}\right] \equiv\left[\begin{array}{ll}a & c \\ c & b\end{array}\right] * /$

$\zeta=-h y p *(b+h y p * a) /(2 c)$

$t=\operatorname{sign}(\zeta) /\left(|\zeta|+\sqrt{\zeta^{2}-h y p}\right)$

$h=\sqrt{1-h y p * t^{2}}$

$c s=1 / h$

$s n=t / h$

$s n 1=h y p * s n$

${ }^{*}$ update the 2 by 2 pivot submatrix */

$H_{i i}=a+h y p * c * t$

$H_{j j}=b+c * t$

$H_{i j}=H_{j i}=0$

${ }^{*}$ update the rest of rows and columns $i$ and $j * /$

for $k=1$ to $n$ except $i$ and $j$

tmp $=H_{i k}$

$H_{i k}=c s * t m p+s n 1 * H_{j k}$

$H_{j k}=s n * t m p+c s * H_{j k}$

$H_{k i}=H_{i k}$

$H_{k j}=H_{j k}$

endfor

${ }^{*}$ update the eigenvector matrix $V *$ /

for $k=1$ to $n$

tmp $=V_{k i}$

$V_{k i}=c s * t m p+s n 1 * V_{k j}$

$V_{k j}=s n * t m p+c s * V_{k j}$

endfor

endfor

until convergence $\left(\right.$ all $\left|H_{i j}\right| /\left(H_{i i} H_{j j}\right)^{1 / 2} \leq$ tol $)$

${ }^{*}$ the computed eigenvalues of the pair $H, J$ are $\lambda_{j}=H_{j j} J_{j j} *$ / 


\section{${ }^{*}$ the computed eigenvectors of the pair $H, J$ are the columns of the final matrix $V *$ /}

Our algorithm is essentially the standard one introduced by Rutishauser [22]. The formulae for the hyperbolic case are derived in the same manner as for the trigonometric one [29]. In the following section we analyse this (simple) version of the algorithm. We omitt enhancements like delayed updates of the diagonals and fast rotations, to make the analysis clearer. Analysis of the fast rotations is given for the implicit method in Sect. 3.4. One of the differences between our algorithm and the standard one is the stopping criterion. This criterion is also used in [13, 29, 31]. Our justification of this criterion is the same as in [13]: according to Th. 2.2.1, the accuracy of the eigenvalues depends on $1 / \lambda_{\min }(A)$ (or $\left.\kappa(A)\right)$ and not on $\kappa(H)$, so that we set $H_{i j}$ to zero only if $\left|H_{i j}\right| /\left(H_{i i} H_{j j}\right)^{1 / 2}$ is small, not just if $\left|H_{i j}\right| / \max _{k l}\left|H_{k l}\right|$ is small.

One difference between trigonometric and hyperbolic rotations is that $\operatorname{Tr}\left(H^{\prime}\right)=$ $\operatorname{Tr}(H)$ after trigonometric, and $\operatorname{Tr}\left(H^{\prime}\right)<\operatorname{Tr}(H)$ after hyperbolic rotation. Using this trace reduction argument Veselić [29] proved that the hyperbolic parameter $t$ tends to zero. The second difference is that the condition of the transformation matrix is in the trigonometric case one, while in the hyperbolic case it can be large. Note, however, that

$$
|\tanh y| \leq \frac{\sqrt{\kappa(A)}-1}{\sqrt{\kappa(A)}+1},
$$

where $A$ is the scaled matrix, i.e. $H=D A D$, $\operatorname{diag}(A)=I$. Moreover, if $G, J$ is the output of the symmetric indefinite decomposition, then the scaled condition of the matrix $G^{T} G$ is generally small (see Sect. 4.4, Chap. 5), and it does not grow much during the Jacobi process (see Sect. 3.2.2, Chap. 5), so the hyperbolic parameters are generally moderate. In Subsect. 3.2.1 we show how to modify hyperbolic rotations in order to bound the condition of the transformation matrix. This modification improves the theoretical bounds, but it does not seem to be of importance in practice.

Veselic [29] proved that the $J$-orthogonal Jacobi method is globally convergent for the optimal strategy, threshold strategies, row-cyclic strategy, and all other strategies which are equivalent to the row-cyclic one (for example, the modulus parallel strategy [18]). He also proved a very interesting fact that all $J$-orthogonal matrices $V$ which satisfy (3.1.2) have the same condition number. Moreover, if $V_{1}$ and $V_{2}$ are two such matrices, then

$$
V_{2}=V_{1} U, \quad U=\left[\begin{array}{cc}
U_{1} & 0 \\
0 & U_{2}
\end{array}\right],
$$

where $U_{1}, U_{2}$ are othogonal matrices of order $m, n-m$, respectively.

Drmač and Hari [15] proved that the $J$-orthogonal Jacobi method is quadratically convergent.

œ 


\subsection{Error bounds for the eigenvalues}

In this section we prove that the two-sided $J$-orthogonal Jacobi method in floatingpoint arithmetic applied to the problem (3.1.1) computes eigenvalues with the error bounds of Chap. 2. Since the computed eigenvector matrix is not orthogonal and is not needed when applying our algorithm to a single indefinite matrix, we do not investigate the accuracy of the computed eigenvectors.

Let $H_{0}=D_{0} A_{0} D_{0}$ be the initial matrix, and $H_{m}=D_{m} A_{m} D_{m}$ where $H_{m}$ is obtained from $H_{m-1}$ by applying a single $J$-orthogonal Jacobi rotation. Here $D_{m}$ is diagonal and $A_{m}$ has unit diagonal as before. All the error bounds in this section contain the quantities $1 / \lambda_{\min }(A)$ (or $\kappa\left(A_{m}\right)$ ), whereas the perturbation bounds of Chap. 2 are proportional to $\kappa\left(A_{0}\right)$. Therefore, our claim that $J$-orthogonal Jacobi method solves the eigenproblem as accurately as predicted in Chap. 2 depends, as in [13], on the ratios $\max _{m} \lambda_{\text {min }}\left(A_{0}\right) / \lambda_{\text {min }}\left(A_{m}\right)\left(\right.$ or $\max _{m} \kappa\left(A_{m}\right) / \kappa\left(A_{0}\right)$ ) being modest in size. Note that the convergence of $H_{m}$ to diagonal form is equivalent to the convergence of $A_{m}$ to the identity, or $\kappa\left(A_{m}\right)$ to 1 . Thus we expect $\kappa\left(A_{m}\right)$ to be less than $\kappa\left(A_{0}\right)$ eventually. Demmel and Veselić [13] have overwhelming numerical evidence that in the positive definite case $(J=I)$ the above ratios are modest in size. Our experiments of Chap. 5 reveal the same for $J \neq I$. Our theoretical understanding of why these ratios are so small is somewhat weaker; we present our theoretical bounds in Subsect. 3.2.2.

The section is organized as follows: we first show that one step of the method satisfies the perturbation bounds of Chap. 2, and that we can extend this result to an overall error bound (modulo the assumption that the quotients $\max _{m} \lambda_{\min }\left(A_{0}\right) / \lambda_{\min }\left(A_{m}\right)$ are modest). In Subsect. 3.2.1 we show how to modify the method in order to bound potentially large hyperbolic angles, which, in turn, results in better error bounds.

We now present our model of the finite precision floating-point arithmetic. The floating-point result $f l(\cdot)$ of the operation $(\cdot)$ is given by $[33,13]$

$$
\begin{aligned}
f l(a \pm b) & =a\left(1+\varepsilon_{1}\right) \pm b\left(1+\varepsilon_{2}\right) \\
f l(a \times b) & =(a \times b)\left(1+\varepsilon_{3}\right) \\
f l(a / b) & =(a / b)\left(1+\varepsilon_{4}\right) \\
f l(\sqrt{a}) & =\sqrt{a}\left(1+\varepsilon_{5}\right)
\end{aligned}
$$

where $\left|\varepsilon_{i}\right| \leq \varepsilon$, and $\varepsilon \ll 1$ is the machine precision. This is somewhat more general than the usual model which uses $f l(a \pm b)=(a \pm b)\left(1+\varepsilon_{1}\right)$ and includes machines like the Cray which do not have a guard digit. This does not greatly complicate the error analysis, but it is possible that the computed rotation angle may be less accurate. This may adversely affect convergence, but as we will see it does not affect the one-step error analysis.

Numerically subscripted $\varepsilon$ 's will denote independent quantities bounded in magnitude by $\varepsilon$. As usual (e.g. [13]), we will make approximations like $\left(1+i \varepsilon_{1}\right)\left(1+j \varepsilon_{2}\right)=$ $1+(i+j) \varepsilon_{3}$ and $\left(1+i \varepsilon_{1}\right) /\left(1+j \varepsilon_{2}\right)=1+(i+j) \varepsilon_{3}$. 
The next theorem and its corollary justify our accuracy claims for eigenvalues computed by two-sided $J$-orthogonal Jacobi method .

Theorem 3.2.1 Let $H_{m}$ be the sequence of matrices generated by Algorithm 3.1.1 in floating-point arithmetic with precision $\varepsilon$; that is, $H_{m+1}$ is obtained from $H_{m}$ by applying a single $J$-orthogonal Jacobi rotation. Then the following diagram commutes.

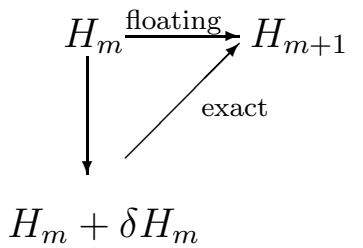

The top arrow indicates that $H_{m+1}$ is obtained from $H_{m}$ by applying one J-orthogonal Jacobi rotation in floating-point arithmetic. The diagonal arrow indicates that $H_{m+1}$ is obtained from $H_{m}+\delta H_{m}$ by applying one J-orthogonal Jacobi rotation in exact arithmetic; thus $H_{m+1}$ amd $H_{m}+\delta H_{m}$ are exactly similar. $\delta H_{m}$ is bounded as follows. Let $\kappa=\kappa\left(A_{m}\right)$, and write $\delta H_{m}=D_{m} \delta A_{m} D_{m}$. Then, with the relative error of order $\varepsilon,^{1}$

$$
\left\|\delta A_{m}\right\|_{2} \leq C_{m} \varepsilon
$$

where

$$
C_{m}= \begin{cases}60+58 \sqrt{n-2} & \text { in trigon. case, } \\ 35.5+(\sqrt{\kappa}+3)(30.93+8.24 \sqrt{n-2}) & \text { in hyperb. case, } \\ 222.42+46.77 \sqrt{n-2} & |\zeta| \leq \frac{3}{2 \sqrt{2}} \\ 225.5+62.45 \sqrt{n-2} & \text { in hyperb. case, }, \quad|\zeta|>\frac{3}{2 \sqrt{2}} \\ & \text { in hyperb. case, }, \quad|\zeta|>\frac{1}{2} a, \\ & b<\frac{1}{2} a .\end{cases}
$$

In other words, one step of Jacobi satisfies the assumptions needed for the perturbation bounds of Sect. 2.2.

The bound (3.2.2) seems to be highly discontinuous at $|\zeta|=3 /(2 \sqrt{2})$. This discontinuity can be removed as decribed in Rem. 3.2.4, or by using the modified method of Subsect. 3.2.1.

\footnotetext{
${ }^{1}$ This formulation is explained after the relation $(1.12)$.
} 
Proof. The proof of the commuting diagram is a tedious computation. We shall prove the diagram separately for the trigonometric and for the hyperbolic case. We assume that multiplications with the parameter hyp in Alg. 3.1.1 have no errors. Write the 2 by 2 submatrix of the current matrix $H_{m}$ as

$$
\widehat{H}_{m}=\left[\begin{array}{ll}
a & c \\
c & b
\end{array}\right] \equiv\left[\begin{array}{cc}
d_{i}^{2} & z d_{i} d_{j} \\
z d_{i} d_{j} & d_{j}^{2}
\end{array}\right]
$$

In both cases we can assume without loss of generality that $a \geq b$. By positive definiteness we have

$$
0<|z| \leq \bar{z} \equiv(\kappa-1) /(\kappa+1)<1 .
$$

Let $a^{\prime}$ and $b^{\prime}$ be the new values of $H_{i i}$ and $H_{j j}$ computed by the algorithm, respectively.

Trigonometric case. This case was analysed by Demmel and Veselić [13]. Our proof is essentially the same as theirs, and we repeat it for the sake of completeness. Small differences in the proof lead to a somewhat better bound for $\left\|\delta A_{m}\right\|_{2}$.

Systematic application of the formulae (3.2.1) shows that

$$
\begin{aligned}
\zeta & =f l((b-a) /(2 * c)) \\
& =\left(1+\varepsilon_{4}\right)\left(\left(\left(1+\varepsilon_{1}\right) b-\left(1+\varepsilon_{2}\right) a\right) /\left(\left(1+\varepsilon_{3}\right) 2 c\right)\right) \\
& =\frac{\left(1+\varepsilon_{4}\right)\left(1+\varepsilon_{2}\right)}{1+\varepsilon_{3}}\left(\frac{\tilde{b}-a}{2 c}\right)
\end{aligned}
$$

where

$$
\widetilde{b} \equiv \frac{1+\varepsilon_{1}}{1+\varepsilon_{2}} b \equiv\left(1+\varepsilon_{b}\right) b, \quad\left|\varepsilon_{b}\right| \leq 2 \varepsilon
$$

Thus

$$
\zeta=\left(1+\varepsilon_{\zeta}\right) \frac{\widetilde{b}-a}{2 c}, \quad\left|\varepsilon_{\zeta}\right| \leq 3 \varepsilon
$$

Let $\widetilde{t}, \widetilde{c s}, \widetilde{s n}$ and $-\widetilde{s n}$ denote the true values of $t, c s, s n$ and $s n 1=-s n$ (i.e. without rounding error) as a function of $a, \widetilde{b}$ and $c$. Using (3.2.1) again one can show that

$$
t=\left(1+\varepsilon_{t}\right) \tilde{t}, \quad c s=\left(1+\varepsilon_{c s}\right) \widetilde{c s}, \quad s n=\left(1+\varepsilon_{s n}\right) \widetilde{s n}
$$

where ${ }^{2}$

$$
\left|\varepsilon_{t}\right| \leq 7 \varepsilon, \quad\left|\varepsilon_{c s}\right| \leq 10 \varepsilon, \quad\left|\varepsilon_{s n}\right| \leq 17 \varepsilon .
$$

$\widetilde{c s}$ and $\widetilde{s n}$ define the exact trigonometric Jacobi rotation

$$
J_{m} \equiv\left[\begin{array}{cc}
\widetilde{c s} & \widetilde{s n} \\
-\widetilde{s n} & \widetilde{c s}
\end{array}\right]
$$

\footnotetext{
${ }^{2}$ Calculating $s n$ as $s n=t / h$ instead of $s n=t \cdot c s$ [13] saves one $\varepsilon$ in bounding $\varepsilon_{s n}$. This was noticed by Drmač [14].
} 
which transforms $H_{m}+\delta H_{m}$ to $H_{m+1}$ in the diagram in the statement of the theorem:

$$
J_{m}^{T}\left(H_{m}+\delta H_{m}\right) J_{m}=H_{m+1} .
$$

Now we begin constructing $\delta H_{m} . \quad \delta H_{m}$ will be nonzero only in the rows and columns $i$ and $j$. We first compute its entries outside the 2 by 2 pivot submatrix. Let $H_{i k}^{\prime}$ and $H_{j k}^{\prime}$ denote the updated quantities computed by the algorithm. Then

$$
\begin{aligned}
H_{i k}^{\prime} & =f l\left(c s * H_{i k}-s n * H_{j k}\right) \\
& =\left(1+\varepsilon_{4}\right)\left(1+\varepsilon_{5}\right) c s H_{i k}-\left(1+\varepsilon_{6}\right)\left(1+\varepsilon_{7}\right) s n H_{j k} \\
& =\left(1+\varepsilon_{4}\right)\left(1+\varepsilon_{5}\right)\left(1+\varepsilon_{c s}\right) \widetilde{c s} H_{i k}-\left(1+\varepsilon_{6}\right)\left(1+\varepsilon_{7}\right)\left(1+\varepsilon_{s n}\right) \widetilde{s n} H_{j k} \\
& \equiv \widetilde{c s} H_{i k}-\widetilde{s n} H_{j k}+\epsilon\left(H_{i k}^{\prime}\right),
\end{aligned}
$$

where

$$
\epsilon\left(H_{i k}^{\prime}\right)=\varepsilon_{1}^{\prime} \widetilde{c s} H_{i k}-\varepsilon_{2}^{\prime} \widetilde{s n} H_{j k}, \quad\left|\varepsilon_{1}^{\prime}\right| \leq 12 \varepsilon,\left|\varepsilon_{2}^{\prime}\right| \leq 19 \varepsilon
$$

Similarly,

$$
\begin{aligned}
H_{j k}^{\prime} & =f l\left(s n * H_{i k}+c s * H_{j k}\right) \\
& =\widetilde{s n} H_{i k}+\widetilde{c s} H_{j k}+\epsilon\left(H_{j k}^{\prime}\right),
\end{aligned}
$$

where

$$
\epsilon\left(H_{j k}^{\prime}\right)=\varepsilon_{3}^{\prime} \widetilde{c s} H_{j k}+\varepsilon_{4}^{\prime} \widetilde{s n} H_{i k}, \quad\left|\varepsilon_{3}^{\prime}\right| \leq 12 \varepsilon,\left|\varepsilon_{4}^{\prime}\right| \leq 19 \varepsilon
$$

Thus

$$
\begin{aligned}
{\left[\begin{array}{c}
H_{i k}^{\prime} \\
H_{j k}^{\prime}
\end{array}\right] } & =J_{m}^{T}\left[\begin{array}{l}
H_{i k} \\
H_{j k}
\end{array}\right]+\left[\begin{array}{c}
\epsilon\left(H_{i k}\right) \\
\epsilon\left(H_{j k}\right)
\end{array}\right] \\
& =J_{m}^{T}\left(\left[\begin{array}{c}
H_{i k} \\
H_{j k}
\end{array}\right]+J_{m}\left[\begin{array}{c}
\epsilon\left(H_{i k}\right) \\
\epsilon\left(H_{j k}\right)
\end{array}\right]\right) \\
& \equiv J_{m}^{T}\left(\left[\begin{array}{c}
H_{i k} \\
H_{j k}
\end{array}\right]+\left[\begin{array}{c}
\delta H_{i k} \\
\delta H_{j k}
\end{array}\right]\right),
\end{aligned}
$$

where

$$
\begin{aligned}
& \delta H_{i k}=\varepsilon_{1}^{\prime} \widetilde{c s}^{2} H_{i k}-\varepsilon_{2}^{\prime} \widetilde{c s} \widetilde{s n} H_{j k}+\varepsilon_{3}^{\prime} \widetilde{c s} \widetilde{s n} H_{j k}+\varepsilon_{4}^{\prime} \widetilde{s n} H_{i k} \\
& \delta H_{j k}=-\varepsilon_{1}^{\prime} \widetilde{c s s n} H_{i k}+\varepsilon_{2}^{\prime} \widetilde{s n}^{2} H_{j k}+\varepsilon_{3}^{\prime} \widetilde{c s}^{2} H_{j k}+\varepsilon_{4}^{\prime} \widetilde{c s} \widetilde{s n} H_{i k}
\end{aligned}
$$

Using

$$
\left|H_{i j}\right| \leq d_{i} d_{j}, \quad \widetilde{c s}=\frac{1}{\sqrt{1+\widetilde{t}^{2}}}, \quad \widetilde{s n}=\frac{\widetilde{t}}{\sqrt{1+\widetilde{t}^{2}}}
$$

we have

$$
\left|\delta A_{i k}\right| \leq \frac{1}{1+\widetilde{t}^{2}}\left(12+31|\widetilde{t}| \frac{d_{j}}{d_{i}}+19 \widetilde{t}^{2}\right) \varepsilon,
$$


which is an increasing function for $|\widetilde{t}| \in[0,1]$.

Set $x \equiv d_{j} / d_{i}$. Note that $x \leq 1$. In estimating $\left|\delta A_{j k}\right|$ we consider two cases: $x<\bar{x} \equiv .48$, and $x \geq \bar{x}$. If $x<\bar{x}$, then, with the relative error of $O(\varepsilon)$, we have

$$
|\widetilde{t}|=\frac{1}{\frac{1-x^{2}}{2|z| x}+\left(1+\left(\frac{1-x^{2}}{2|z| x}\right)^{2}\right)^{1 / 2}} \leq \frac{x}{1-\bar{x}^{2}} .
$$

Since we want to bound $\left|\delta A_{j k}\right|$ with a bound of order $\varepsilon$, we neglect the relative error of $O(\varepsilon)$ in the above inequality. Therefore,

$$
\left|\delta A_{j k}\right| \leq \frac{1}{1+\widetilde{t}^{2}}\left(12+31 \frac{1}{1-\bar{x}^{2}}+19 \widetilde{t}^{2}\right) \varepsilon
$$

which is a decreasing function of $\widetilde{t}^{2}$. Substituting 1 for $\widetilde{t}$ and $\bar{x}$ for $d_{j} / d_{i}$ in (3.2.5), and 0 for $\tilde{t}$ in (3.2.6), we obtain

$$
\sqrt{\delta A_{i k}^{2}+\delta A_{j k}^{2}} \leq 57.3 \varepsilon
$$

If $x \geq \bar{x}$, then

$$
\left|\delta A_{j k}\right| \leq \frac{1}{1+\widetilde{t}^{2}}\left(12+31|\widetilde{t}| \frac{1}{\bar{x}}+19 \widetilde{t}^{2}\right) \varepsilon,
$$

which is an increasing function of $|\widetilde{t}| \in[0,1]$. Substituting 1 for $\widetilde{t}$ and $d_{j} / d_{i}$ in (3.2.5) and (3.2.8), we obtain

$$
\sqrt{\delta A_{i k}^{2}+\delta A_{j k}^{2}} \leq 57.4 \varepsilon
$$

Note that our choice of $\bar{x}$ makes bounds in relations (3.2.7) and (3.2.9) almost equal.

Now we construct the 2 by 2 submatrix $\delta \widehat{H}_{m}$ of $\delta H_{m}$ at the intersection of the rows and columns $i$ and $j$. We will construct it of three components

$$
\delta \widehat{H}_{m}=\Delta_{1}+\Delta_{2}+\Delta_{3} .
$$

Applying the relations (3.2.1), we obtain

$$
\begin{aligned}
b^{\prime} & =f l(b+c t)=\frac{1+\varepsilon_{2}}{1+\varepsilon_{1}}\left(1+\varepsilon_{8}\right) \tilde{b}+\left(1+\varepsilon_{9}\right)\left(1+\varepsilon_{10}\right)\left(1+\varepsilon_{t}\right) c \tilde{t} \\
& =\left(1+\varepsilon_{9}\right)\left(1+\varepsilon_{10}\right)\left(1+\varepsilon_{t}\right)\left(\frac{\left(1+\varepsilon_{2}\right)\left(1+\varepsilon_{8}\right)}{\left(1+\varepsilon_{1}\right)\left(1+\varepsilon_{9}\right)\left(1+\varepsilon_{10}\right)\left(1+\varepsilon_{t}\right)} \widetilde{b}+c \tilde{t}\right) \\
& \equiv\left(1+\varepsilon_{b^{\prime}}\right)\left(\widetilde{b}+c \tilde{t}+\varepsilon_{b}^{\prime} \tilde{b}\right),
\end{aligned}
$$

where $\left|\varepsilon_{b^{\prime}}\right| \leq 9 \varepsilon$ and $\left|\varepsilon_{b}^{\prime}\right| \leq 12 \varepsilon$. Similarly,

$$
\begin{aligned}
a^{\prime} & =f l(a-c t)=\left(1+\varepsilon_{11}\right) a-\left(1+\varepsilon_{12}\right)\left(1+\varepsilon_{13}\right)\left(1+\varepsilon_{t}\right) c \widetilde{t} \\
& =\left(1+\varepsilon_{a^{\prime}}\right)(a-c \widetilde{t})
\end{aligned}
$$


where $\left|\varepsilon_{a^{\prime}}\right| \leq 9 \varepsilon$. Here we used the fact that $c \tilde{t}<0$.

Now let

$$
\Delta_{1}=\left[\begin{array}{cc}
0 & 0 \\
0 & \varepsilon_{b} b
\end{array}\right]+J_{m}\left[\begin{array}{cc}
0 & 0 \\
0 & \varepsilon_{b}^{\prime} \widetilde{b}
\end{array}\right] J_{m}^{T} .
$$

From earlier discussion we see that

$$
J_{m}^{T}\left(\left[\begin{array}{ll}
a & c \\
c & b
\end{array}\right]+\Delta_{1}\right) J_{m}=\left[\begin{array}{cc}
a-c \widetilde{t} & 0 \\
0 & b+c \tilde{t}+\varepsilon_{b}^{\prime} \tilde{b}
\end{array}\right] .
$$

Next let

$$
\Delta_{2}=\varepsilon_{a^{\prime}}\left(\left[\begin{array}{ll}
a & c \\
c & b
\end{array}\right]+\Delta_{1}\right)
$$

Thus

$$
\begin{aligned}
J_{m}^{T}\left(\left[\begin{array}{ll}
a & c \\
c & b
\end{array}\right]+\Delta_{1}+\Delta_{2}\right) J_{m} & =\left(1+\varepsilon_{a^{\prime}}\right)\left[\begin{array}{cc}
a-c \tilde{t} & 0 \\
0 & b+c \tilde{t}+\varepsilon_{b}^{\prime} \tilde{b}
\end{array}\right] \\
& =\left[\begin{array}{cc}
a^{\prime} & 0 \\
0 & b^{\prime} \frac{1+\varepsilon_{a^{\prime}}}{1+\varepsilon_{b^{\prime}}}
\end{array}\right] .
\end{aligned}
$$

Now let

$$
\Delta_{3}=J_{m}\left[\begin{array}{cc}
0 & 0 \\
0 & b^{\prime}\left(1-\frac{1+\varepsilon_{a^{\prime}}}{1+\varepsilon_{b^{\prime}}}\right)
\end{array}\right] J_{m}^{T} \equiv\left[\begin{array}{cc}
\widetilde{s n}^{2} \varepsilon_{b^{\prime \prime}} b^{\prime} & \widetilde{c s} \widetilde{s n} \varepsilon_{b^{\prime \prime}} b^{\prime} \\
\widetilde{c s} \widetilde{s n} \varepsilon_{b^{\prime \prime}} b^{\prime} & \widetilde{c s} \varepsilon_{b^{\prime \prime}} b^{\prime}
\end{array}\right],
$$

where $\left|\varepsilon_{b^{\prime \prime}}\right| \leq\left|\varepsilon_{a^{\prime}}\right|+\left|\varepsilon_{b^{\prime}}\right| \leq 18 \varepsilon$. Then

$$
J_{m}^{T}\left(\left[\begin{array}{cc}
a & c \\
c & b
\end{array}\right]+\Delta_{1}+\Delta_{2}+\Delta_{3}\right) J_{m}=\left[\begin{array}{cc}
a^{\prime} & 0 \\
0 & b^{\prime}
\end{array}\right]
$$

as desired. This completes the construction of $\delta \widehat{H}_{m}$. Since $\widetilde{b}=b\left(1+\varepsilon_{b}\right)$ and $b^{\prime}<b$,

$$
\left\|\delta \widehat{A}_{m}\right\|_{2} \leq\left|\varepsilon_{b}\right|+\left|\varepsilon_{b}^{\prime}\right|+2 \cdot\left|\varepsilon_{a^{\prime}}\right|+\left|\varepsilon_{b^{\prime \prime}}\right| \leq 60 \varepsilon
$$

holds with the relative error of $O(\varepsilon)$. From (3.2.7), (3.2.9), and the above relation, it finally follows

$$
\left\|\delta A_{m}\right\|_{2} \leq(60+58 \sqrt{n-2}) \varepsilon .
$$

This bound improves the bound $\left\|\delta A_{m}\right\|_{2} \leq(257 \sqrt{n-2}+104) \varepsilon$ from [13].

$æ$ 
Hyperbolic case. To avoid the confusion with the trigonometric case, we denote the quantities $c s$, sn and $s n 1=s n$ computed by Alg. 3.1.1 with $c h$ and $s h$, respectively. We first compute $\widetilde{t}, \widetilde{h}, \widetilde{c h}$ and $\widetilde{s h}$ as the exact values of the parameters computed without rounding errors from $a, b$ and some $\widetilde{c} \equiv\left(1+\varepsilon_{c}\right) c$. Generally, the following bounds hold:

$$
\begin{array}{r}
|\zeta| \geq \frac{\kappa+1}{\kappa-1}>1, \\
|t| \leq \frac{\sqrt{\kappa}-1}{\sqrt{\kappa}+1}<1, \\
|s h|<c h \leq \frac{1}{2}\left(\sqrt[4]{\kappa}+\frac{1}{\sqrt[4]{\kappa}}\right) .
\end{array}
$$

Let $\zeta_{0} \equiv(a+b) /(-2 c)$ be the exact value of $(a+b) /(-2 c)$. Systematic application of (3.2.1) gives

$$
\zeta_{1}=f l\left(\frac{a+b}{-2 c}\right)=\left(1+\varepsilon_{\zeta_{1}}\right) \zeta_{0}
$$

where $\left|\varepsilon_{\zeta_{1}}\right| \leq 3 \varepsilon$, and

$$
\begin{aligned}
t_{1} & =f l\left(\frac{\operatorname{sign}\left(\zeta_{1}\right)}{\left|\zeta_{1}\right|+\sqrt{\zeta_{1}^{2}-1}}\right) \\
& =\frac{\left(1+\varepsilon_{1}\right) \operatorname{sign}\left(\zeta_{1}\right)}{\left(1+\varepsilon_{2}\right)\left(\left|\zeta_{1}\right|+\left(1+\varepsilon_{3}\right) \sqrt{\zeta_{1}^{2}\left(1+\varepsilon_{4}\right)\left(1+\varepsilon_{5}\right)-\left(1+\varepsilon_{6}\right.}\right)} \\
& =\frac{1+\varepsilon_{1}}{1+\varepsilon_{2}} \cdot \frac{\operatorname{sign}\left(\zeta_{1}\right)}{\left|\zeta_{1}\right|+\left(1+\varepsilon_{3}\right) \sqrt{1+\varepsilon_{6}} \sqrt{\zeta_{1}^{2} \frac{\left(1+\varepsilon_{4}\right)\left(1+\varepsilon_{5}\right)}{1+\varepsilon_{6}}-1}} .
\end{aligned}
$$

Now let

$$
\zeta_{2}^{2} \equiv \zeta_{1}^{2} \frac{\left(1+\varepsilon_{4}\right)\left(1+\varepsilon_{5}\right)}{1+\varepsilon_{6}} .
$$

Then $\zeta_{2} \equiv\left(1+\varepsilon_{\zeta_{2}}\right) \zeta_{1}$, where $\left|\varepsilon_{\zeta_{2}}\right| \leq 1.5 \varepsilon$. This implies that

$$
\begin{aligned}
t_{1} & =\frac{1+\varepsilon_{1}}{1+\varepsilon_{2}} \cdot \frac{\operatorname{sign}\left(\zeta_{1}\right)}{\left|\zeta_{2} \frac{1}{1+\varepsilon_{\zeta_{2}}}\right|+\left(1+\varepsilon_{3}\right) \sqrt{1+\varepsilon_{6}} \sqrt{\zeta_{2}^{2}-1}} \\
& \equiv\left(1+\varepsilon_{t_{1}}\right) \frac{\operatorname{sign}\left(\zeta_{2}\right)}{\left|\zeta_{2}\right|+\sqrt{\zeta_{2}^{2}-1}},
\end{aligned}
$$

where $\left|\varepsilon_{t_{1}}\right| \leq 3.5 \varepsilon$. Furthermore,

$$
h=f l\left(\sqrt{1-t_{1}^{2}}\right)=\left(1+\varepsilon_{7}\right) \sqrt{\left(1+\varepsilon_{8}\right)-\left(1+\varepsilon_{9}\right)\left(1+\varepsilon_{10}\right) t_{1}^{2}}
$$




$$
\begin{aligned}
& =\left(1+\varepsilon_{7}\right) \sqrt{1+\varepsilon_{8}} \sqrt{1-\frac{\left(1+\varepsilon_{9}\right)\left(1+\varepsilon_{10}\right)}{1+\varepsilon_{8}} t_{1}^{2}} \\
& \equiv\left(1+\varepsilon_{h}\right) \sqrt{1-t_{2}^{2}}
\end{aligned}
$$

where

$$
\left|\varepsilon_{h}\right| \leq 1.5 \varepsilon, \quad t_{2}^{2} \equiv \frac{\left(1+\varepsilon_{9}\right)\left(1+\varepsilon_{10}\right)}{1+\varepsilon_{8}} t_{1}^{2},
$$

i.e.

$$
t_{2}=\left(1+\varepsilon_{t_{2}}\right) t_{1}, \quad\left|\varepsilon_{t_{2}}\right| \leq 1.5 \varepsilon
$$

Therefore,

$$
\frac{\operatorname{sign}\left(\zeta_{2}\right)}{\left|\zeta_{2}\right|+\sqrt{\zeta_{2}^{2}-1}}=\frac{1}{1+\varepsilon_{t_{1}}} t_{1}=\frac{1}{\left(1+\varepsilon_{t_{1}}\right)\left(1+\varepsilon_{t_{2}}\right)} t_{2} \equiv\left(1+\varepsilon_{t_{2}}^{\prime}\right) t_{2},
$$

where $\left|\varepsilon_{t_{2}}^{\prime}\right| \leq\left|\varepsilon_{t_{1}}\right|+\left|\varepsilon_{t_{2}}\right| \leq 5 \varepsilon$. In exact arithmetic

$$
t=\frac{\operatorname{sign}(\zeta)}{|\zeta|+\sqrt{\zeta^{2}-1}}
$$

implies

$$
\zeta=\frac{1}{2}\left(t+\frac{1}{t}\right)
$$

Therefore, in exact arithmetic for $\zeta_{2}$ we have

$$
\zeta_{2}=\frac{1}{2}\left(\left(1+\varepsilon_{t_{2}}^{\prime}\right) t_{2}+\frac{1}{\left(1+\varepsilon_{t_{2}}^{\prime}\right) t_{2}}\right)
$$

which, in turn, implies

$$
\frac{1}{2}\left(t_{2}+\frac{1}{t_{2}}\right)=\left(1+\varepsilon_{\zeta_{2}}^{\prime}\right) \zeta_{2}=\left(1+\varepsilon_{\zeta_{2}}^{\prime}\right)\left(1+\varepsilon_{\zeta_{2}}\right) \zeta_{0} \equiv\left(1+\varepsilon_{\zeta_{2}}^{\prime \prime}\right) \zeta_{0},
$$

where

$$
\left|\varepsilon_{\zeta_{2}}^{\prime}\right| \leq\left|\varepsilon_{t_{2}}^{\prime}\right| \leq 5 \varepsilon, \quad\left|\varepsilon_{\zeta_{2}}^{\prime \prime}\right| \leq\left|\varepsilon_{\zeta_{2}}^{\prime}\right|+\left|\varepsilon_{\zeta_{2}}\right| \leq 9.5 \varepsilon .
$$

Therefore, we can choose $\varepsilon_{c}$,

$$
\left|\varepsilon_{c}\right| \leq\left|\varepsilon_{\zeta_{2}}^{\prime \prime}\right| \leq 9.5 \varepsilon
$$

such that for $\widetilde{c}=\left(1+\varepsilon_{c}\right) c$ in exact $\operatorname{arithmetic~}^{3}$

$$
\frac{a+b}{-2 \widetilde{c}}=\left(1+\varepsilon_{\zeta_{2}}^{\prime \prime}\right) \zeta_{0}
$$

\footnotetext{
${ }^{3}$ In the trigonometric case we had to perturb $b$. Here we can perturb either $a$ or $c$, and we perturb $c$ since it is absolutely smaller.
} 
From (3.2.14) and (3.2.15) it follows that $\widetilde{t}=t_{2}$ is the exact value of the parameter $t$ computed without rounding errors from $a, b$ and $\widetilde{c}$. Set

$$
\widetilde{h}=\sqrt{1-\widetilde{t}^{2}}, \quad \widetilde{c h}=\frac{1}{\widetilde{h}}, \quad \widetilde{s h}=\frac{\widetilde{t}}{\widetilde{h}} .
$$

For the computed quantities we have

$$
\begin{aligned}
t & =t_{1}=\left(1+\varepsilon_{t}\right) \widetilde{t}, \quad\left|\varepsilon_{t}\right| \leq\left|\varepsilon_{t_{2}}\right| \leq 1.5 \varepsilon, \\
h & =\left(1+\varepsilon_{h}\right) \widetilde{h}, \quad\left|\varepsilon_{h}\right| \leq 1.5 \varepsilon, \\
c h & =f l(1 / h)=\left(1+\varepsilon_{c h}\right) \widetilde{c h}, \quad\left|\varepsilon_{c h}\right| \leq \varepsilon+\left|\varepsilon_{h}\right| \leq 2.5 \varepsilon, \\
s h & =f l(t / h)=\left(1+\varepsilon_{s h}\right) \widetilde{s h}, \quad\left|\varepsilon_{s h}\right| \leq \varepsilon+\left|\varepsilon_{t}\right|+\left|\varepsilon_{h}\right| \leq 4 \varepsilon .
\end{aligned}
$$

Here the first line follows from $\tilde{t}=t_{2}$ and (3.2.12), the second line follows from (3.2.13), and the last two lines follow from the first two lines and the formulae (3.2.1). $\widetilde{c h}$ and $\widetilde{s h}$ define the exact hyperbolic rotation

$$
J_{m} \equiv\left[\begin{array}{cc}
\widetilde{c h} & \widetilde{s h} \\
\widetilde{s h} & \widetilde{c h}
\end{array}\right]
$$

which transforms $H_{m}+\delta H_{m}$ to $H_{m+1}$ in the diagram in the statement of the theorem:

$$
J_{m}\left(H_{m}+\delta H_{m}\right) J_{m}=H_{m+1} .
$$

Now we begin constructing $\delta H_{m} . \quad \delta H_{m}$ will be nonzero only in the rows and columns $i$ and $j$. First we compute its entries outside the 2 by $2(i, j)$ submatrix. Let $H_{i k}^{\prime}$ and $H_{j k}^{\prime}$ denote the updated quantities computed by the algorithm. Then, similarly to the trigonometric case, we have

$$
H_{i k}^{\prime}=f l\left(c h * H_{i k}+s h * H_{j k}\right)=\widetilde{c h} H_{i k}+\widetilde{s h} H_{j k}+\epsilon\left(H_{i k}^{\prime}\right),
$$

where

$$
\epsilon\left(H_{i k}^{\prime}\right)=\varepsilon_{1}^{\prime} \widetilde{c h} H_{i k}+\varepsilon_{2}^{\prime} \widetilde{s h} H_{j k}, \quad\left|\varepsilon_{1}^{\prime}\right| \leq 4.5 \varepsilon,\left|\varepsilon_{2}^{\prime}\right| \leq 6 \varepsilon,
$$

and

$$
H_{j k}^{\prime}=f l\left(s h * H_{i k}+c h * H_{j k}\right)=\widetilde{s h} H_{i k}+\widetilde{c h} H_{j k}+\epsilon\left(H_{j k}^{\prime}\right),
$$

where

$$
\epsilon\left(H_{j k}^{\prime}\right)=\varepsilon_{3}^{\prime} \widetilde{\operatorname{ch}} H_{j k}+\varepsilon_{4}^{\prime} \widetilde{s h} H_{i k}, \quad\left|\varepsilon_{3}^{\prime}\right| \leq 4.5 \varepsilon,\left|\varepsilon_{4}^{\prime}\right| \leq 6 \varepsilon .
$$

Thus

$$
\begin{aligned}
{\left[\begin{array}{c}
H_{i k}^{\prime} \\
H_{j k}^{\prime}
\end{array}\right] } & =J_{m}^{T}\left[\begin{array}{l}
H_{i k} \\
H_{j k}
\end{array}\right]+\left[\begin{array}{c}
\epsilon\left(H_{i k}\right) \\
\epsilon\left(H_{j k}\right)
\end{array}\right] \\
& =J_{m}^{T}\left(\left[\begin{array}{c}
H_{i k} \\
H_{j k}
\end{array}\right]+J_{m}^{-1}\left[\begin{array}{c}
\epsilon\left(H_{i k}\right) \\
\epsilon\left(H_{j k}\right)
\end{array}\right]\right) \\
& \equiv J_{m}\left(\left[\begin{array}{c}
H_{i k} \\
H_{j k}
\end{array}\right]+\left[\begin{array}{c}
\delta H_{i k} \\
\delta H_{j k}
\end{array}\right]\right),
\end{aligned}
$$


where

$$
\begin{aligned}
& \delta H_{i k}=\varepsilon_{1}^{\prime} \widetilde{c h}^{2} H_{i k}+\varepsilon_{2}^{\prime} \widetilde{\operatorname{ch} \widetilde{s h}} H_{j k}-\varepsilon_{3}^{\prime} \widetilde{\operatorname{ch} \widetilde{s h}} H_{j k}-\varepsilon_{4}^{\prime} \widetilde{\operatorname{sh}}^{2} H_{i k}, \\
& \delta H_{j k}=-\varepsilon_{1}^{\prime} \widetilde{\operatorname{ch} s h} H_{i k}-\varepsilon_{2}^{\prime} \widetilde{s h}^{2} H_{j k}+\varepsilon_{3}^{\prime} \widetilde{c h}^{2} H_{j k}+\varepsilon_{4}^{\prime} \widetilde{\operatorname{ch} s h} H_{i k} .
\end{aligned}
$$

$æ$ 
Contrary to the trigonometric case, we analyse two cases. The first case is when $|\zeta|$ is near the bound (3.2.11), and the second case is when $|\zeta|$ is bounded away from (3.2.11). Set, as in the trigonometric case, $x \equiv d_{j} / d_{i}$. Set ${ }^{4}$

$$
\alpha=\frac{3}{2 \sqrt{2}}, \quad \beta \equiv \frac{1}{\alpha+\sqrt{\alpha^{2}-1}}=\frac{1}{\sqrt{2}} .
$$

Case $I .|\zeta| \leq \alpha$.

From our assumption, the definition of $\zeta$, and $|c| \leq \sqrt{a b}$ it follows

$$
a+b \leq \alpha \cdot 2 \sqrt{a b},
$$

i.e.

This implies

$$
\frac{1}{x}+x \leq 2 \alpha
$$

$$
\begin{aligned}
x & \geq \frac{1}{\alpha+\sqrt{\alpha^{2}-1}} \equiv \beta=\frac{1}{\sqrt{2}}, \\
d_{j} \leq d_{i} & \leq \frac{1}{\beta} d_{j}, \quad b \leq a \leq \frac{1}{\beta^{2}} b,
\end{aligned}
$$

i.e. when $|\zeta|$ is near its lower bound, then $a$ and $b$ do not differ much. We now show that

$$
\widetilde{c h} \leq \frac{1}{2}\left(\sqrt[4]{\kappa}+\frac{1}{\sqrt[4]{\kappa}}\right)
$$

holds with the relative error of $O(\varepsilon)$. Indeed, $c=z d_{i} d_{j}$ implies $\widetilde{c}=\widetilde{z} d_{i} d_{j}=z(1+$ $\left.\varepsilon_{c}\right) d_{i} d_{j}$. Set

$$
\kappa_{1} \equiv\left(1+z\left(1+\varepsilon_{c}\right)\right) /\left(1-z\left(1+\varepsilon_{c}\right)\right)
$$

Then

$$
\widetilde{c h} \leq\left(\sqrt[4]{\kappa_{1}}+\frac{1}{\sqrt[4]{\kappa_{1}}}\right) / 2
$$

A simple calculation shows that

$$
1-z\left(1+\varepsilon_{c}\right)=\left(1+\varepsilon^{\prime}\right)(1-z), \quad\left|\varepsilon^{\prime}\right| \leq\left|\varepsilon_{c}\right|(\kappa+1) / 2 .
$$

Therefore,

$$
\kappa_{1} \equiv \frac{1+z\left(1+\varepsilon_{c}\right)}{1-z\left(1+\varepsilon_{c}\right)}=\frac{1+z}{1-z}\left(1+\varepsilon_{c}\right)\left(1+\varepsilon^{\prime}\right) \leq \kappa\left(1+\left|\varepsilon_{c}\right|+\left|\varepsilon^{\prime}\right|\right),
$$

and (3.2.19) holds. We neglect the relative error of $O(\varepsilon)$ since it adds only the relative error of $O(\varepsilon)$ in the final estimate. Therefore,

$$
\widetilde{s h}^{2} \leq|\widetilde{s h}| \widetilde{c h} \leq \widetilde{c h}^{2} \leq \frac{1}{4}(\sqrt{\kappa}+3) .
$$

\footnotetext{
${ }^{4}$ Note that we can choose some other $\alpha$, as well. This choice is explained in Rem. 3.2.4 below.
} 
Using the fact that $\left|H_{i j}\right| \leq d_{i} d_{j}$, and inserting (3.2.18) and (3.2.20) into (3.2.16), we obtain

$$
\begin{aligned}
\left|\delta H_{i k}\right| & \leq \frac{1}{4}(\sqrt{\kappa}+3)\left(\left|\varepsilon_{1}^{\prime}\right|+\left|\varepsilon_{2}^{\prime}\right|+\left|\varepsilon_{3}^{\prime}\right|+\left|\varepsilon_{4}^{\prime}\right|\right) d_{i} d_{k} \\
& \leq 5.25(\sqrt{\kappa}+3) d_{i} d_{k} \varepsilon \\
\left|\delta H_{j k}\right| & \leq \frac{1}{4}(\sqrt{\kappa}+3)\left(\left|\varepsilon_{2}^{\prime}\right|+\left|\varepsilon_{3}^{\prime}\right|+\frac{1}{\beta}\left(\left|\varepsilon_{1}^{\prime}\right|+\left|\varepsilon_{4}^{\prime}\right|\right)\right) d_{j} d_{k} \\
& \leq 6.34(\sqrt{\kappa}+3) d_{j} d_{k} \varepsilon .
\end{aligned}
$$

Now we construct the 2 by 2 submatrix $\delta \widehat{H}_{m}$ of $\delta H_{m}$ at the intersection of the rows and columns $i$ and $j$. We will construct it of three components, $\delta \widehat{H}_{m}=\Delta_{1}+\Delta_{2}+\Delta_{3}$. The analysis is somewhat different from the analysis in the trigonometric case because $a^{\prime}<a, b^{\prime}<b$, so that, due to subtraction, $a^{\prime}$ and $b^{\prime}$ can both have large relative errors. We have

$$
\begin{aligned}
a^{\prime} & =f l(a+c t)=\left(1+\varepsilon_{12}\right) a+\left(1+\varepsilon_{13}\right)\left(1+\varepsilon_{14}\right)\left(1+\varepsilon_{c}\right)\left(1+\varepsilon_{t}\right) \tilde{c} \tilde{t} \\
& =\left(1+\varepsilon_{13}\right)\left(1+\varepsilon_{14}\right)\left(1+\varepsilon_{c}\right)\left(1+\varepsilon_{t}\right)\left(\frac{1+\varepsilon_{12}}{\left(1+\varepsilon_{13}\right)\left(1+\varepsilon_{14}\right)\left(1+\varepsilon_{c}\right)\left(1+\varepsilon_{t}\right)} a+\tilde{c} \tilde{t}\right) \\
& =\left(1+\varepsilon_{a^{\prime}}\right)\left(a+\tilde{c} \tilde{t}+\varepsilon_{a} a\right),
\end{aligned}
$$

where

$$
\begin{aligned}
\left|\varepsilon_{a^{\prime}}\right| & \leq 2 \varepsilon+\left|\varepsilon_{c}\right|+\left|\varepsilon_{t}\right| \leq 13 \varepsilon \\
\left|\varepsilon_{a}\right| & \leq 3 \varepsilon+\left|\varepsilon_{c}\right|+\left|\varepsilon_{t}\right| \leq 14 \varepsilon
\end{aligned}
$$

Similarly,

$$
b^{\prime}=f l(b+c t)=\left(1+\varepsilon_{b^{\prime}}\right)\left(b+\widetilde{c} \tilde{t}+\varepsilon_{b} b\right)
$$

where

$$
\left|\varepsilon_{b^{\prime}}\right| \leq 13 \varepsilon, \quad\left|\varepsilon_{b}\right| \leq 14 \varepsilon
$$

Let

$$
\begin{aligned}
\Delta_{1} & =\left[\begin{array}{cc}
0 & \varepsilon_{c} c \\
\varepsilon_{c} c & 0
\end{array}\right]+J_{m}^{-1}\left[\begin{array}{cc}
\varepsilon_{a} a & 0 \\
0 & \varepsilon_{b} b
\end{array}\right] J_{m}^{-1} \\
& =\left[\begin{array}{cc}
0 & \varepsilon_{c} c \\
\varepsilon_{c} c & 0
\end{array}\right]+\left[\begin{array}{cc}
\widetilde{c h}^{2} \varepsilon_{a} a+\widetilde{s h}^{2} \varepsilon_{b} b & -\widetilde{c h} \widetilde{s h}\left(\varepsilon_{a} a+\varepsilon_{b} b\right) \\
\widetilde{c h} \widetilde{s h}\left(\varepsilon_{a} a+\varepsilon_{b} b\right) & \widetilde{s h}^{2} \varepsilon_{a} a+\widetilde{c h}^{2} \varepsilon_{b} b
\end{array}\right],
\end{aligned}
$$

and

$$
\Delta_{2}=\varepsilon_{a^{\prime}}\left(\left[\begin{array}{ll}
a & c \\
c & b
\end{array}\right]+\Delta_{1}\right) .
$$

From earlier discussion we see that

$$
J_{m}\left(\left[\begin{array}{ll}
a & c \\
c & b
\end{array}\right]+\Delta_{1}+\Delta_{2}\right) J_{m}=\left[\begin{array}{cc}
a^{\prime} & 0 \\
0 & b^{\prime} \frac{1+\varepsilon_{a^{\prime}}}{1+\varepsilon_{b^{\prime}}}
\end{array}\right] .
$$


Now let

$$
\Delta_{3}=J_{m}^{-1}\left[\begin{array}{cc}
0 & 0 \\
0 & b^{\prime}\left(1-\frac{1+\varepsilon_{a^{\prime}}}{1+\varepsilon_{b^{\prime}}}\right)
\end{array}\right] J_{m}^{-1}=\left[\begin{array}{cc}
\widetilde{s h}^{2} \varepsilon_{b^{\prime \prime}} b^{\prime} & -\widetilde{c h} \widetilde{s h} \varepsilon_{b^{\prime \prime}} b^{\prime} \\
-\widetilde{c h} \widetilde{s h} \varepsilon_{b^{\prime \prime}} b^{\prime} & \widetilde{c h} \varepsilon_{b^{\prime \prime}}^{2} b^{\prime}
\end{array}\right],
$$

where $\left|\varepsilon_{b^{\prime \prime}}\right| \leq\left|\varepsilon_{a^{\prime}}\right|+\left|\varepsilon_{b^{\prime}}\right| \leq 26 \varepsilon$. Then

$$
J_{m}\left(\left[\begin{array}{cc}
a & c \\
c & b
\end{array}\right]+\Delta_{1}+\Delta_{2}+\Delta_{3}\right) J_{m}=\left[\begin{array}{cc}
a^{\prime} & 0 \\
0 & b^{\prime}
\end{array}\right]
$$

to the first order of $\varepsilon$, as desired. This completes the construction of $\delta \widehat{H}_{m}$ and we have

$$
\begin{aligned}
\left\|\delta \widehat{A}_{m}\right\|_{2} & \leq\left|\varepsilon_{c}\right|+\frac{1}{4}(\sqrt{\kappa}+3) \max \left\{\left|\varepsilon_{a}\right|,\left|\varepsilon_{b}\right|\right\}\left(2 \alpha+1+\frac{1}{\beta^{2}}\right)+2\left|\varepsilon_{a^{\prime}}\right|+\frac{1}{2}(\sqrt{\kappa}+3)\left|\varepsilon_{b^{\prime \prime}}\right| \\
& \leq(35.5+(\sqrt{\kappa}+3) 30.93) \varepsilon .
\end{aligned}
$$

Here we used (3.2.18), (3.2.20), and $b^{\prime}<b$. Combining (3.2.21) with the above relation, we finally obtain

$$
\left\|\delta A_{m}\right\|_{2} \leq(35.5+(\sqrt{\kappa}+3)(30.93+8.24 \sqrt{n-2})) \varepsilon .
$$

$æ$ 
Case II. $|\zeta|>\alpha$.

Our assumption implies

$$
\begin{aligned}
|t| & \leq \beta=\frac{1}{\sqrt{2}} \\
c h & \leq \frac{1}{\sqrt{1-\beta^{2}}}=\sqrt{2}, \\
|s h| & \leq \frac{\beta}{\sqrt{1-\beta^{2}}}=1 .
\end{aligned}
$$

These bounds hold with the relative error of $O(\varepsilon)$ for $\widetilde{t}, \widetilde{s h}$ and $\widetilde{c h}$, as well. We split this case into two subcases.

Subcase IIa. $x \equiv d_{j} / d_{i} \geq \beta$.

The analysis is identical to the analysis in the first case; only the upper bounds for $\widetilde{s h}^{2},|\widetilde{s h}| \widetilde{c h}$ and $\widetilde{c h}^{2}$ are now obtained from (3.2.23) and not from (3.2.20). Therefore,

$$
\begin{aligned}
\left|\delta H_{i k}\right| & \leq\left(2\left|\varepsilon_{1}^{\prime}\right|+\sqrt{2}\left|\varepsilon_{2}^{\prime}\right|+\sqrt{2}\left|\varepsilon_{3}^{\prime}\right|+\left|\varepsilon_{4}^{\prime}\right|\right) d_{i} d_{k} \leq 29.85 d_{i} d_{k} \varepsilon \\
\left|\delta H_{j k}\right| & \leq\left(\sqrt{2} \sqrt{2}\left|\varepsilon_{1}^{\prime}\right|+\left|\varepsilon_{2}^{\prime}\right|+2\left|\varepsilon_{3}^{\prime}\right|+\sqrt{2} \sqrt{2}\left|\varepsilon_{4}^{\prime}\right|\right) d_{j} d_{k} \leq 36 d_{j} d_{k} \varepsilon, \\
\left\|\delta \widehat{A}_{m}\right\|_{2} & \leq\left|\varepsilon_{c}\right|+\max \left\{\left|\varepsilon_{a}\right|,\left|\varepsilon_{b}\right|\right\}\left\|\left[\begin{array}{cc}
3 & 3 \sqrt{2} \\
3 \sqrt{2} & 4
\end{array}\right]\right\|_{2}+2\left|\varepsilon_{a^{\prime}}\right|+\left|\varepsilon_{b^{\prime \prime}}\right|\left\|\left[\begin{array}{cc}
1 & \sqrt{2} \\
\sqrt{2} & 2
\end{array}\right]\right\|_{2} \\
& \leq 222.42 \varepsilon,
\end{aligned}
$$

and, altogether,

$$
\left\|\delta A_{m}\right\|_{2} \leq(222.42+46.77 \sqrt{n-2}) \varepsilon .
$$

Subcase IIb. $x \equiv d_{j} / d_{i}<\beta$.

The above assumption implies $|c|<\beta a$. From

$$
\widetilde{\zeta}=-\left(1+x^{2}\right) /(2 \widetilde{z} x), \quad \widetilde{z}=z\left(1+\varepsilon_{c}\right),
$$

it follows

$$
|\widetilde{t}| \leq 2|\widetilde{z}| x /\left(1+x^{2}\right) \leq 2 x
$$

Here we ignored the relative error of $O(\varepsilon)$ in $z$ and and used the fact that $|\widetilde{z}|<1$. Therefore,

$$
\widetilde{c h}|\widetilde{s h}|=\widetilde{c h}^{2}|\widetilde{t}| \leq 2 \widetilde{c h}^{2} \frac{d_{j}}{d_{i}} .
$$

From (3.2.16), (3.2.23), (3.2.25), and our assumption, it follows

$$
\begin{aligned}
& \left|\delta H_{i k}\right| \leq\left(2\left|\varepsilon_{1}^{\prime}\right|+\sqrt{2}\left|\varepsilon_{2}^{\prime}\right| \frac{1}{\sqrt{2}}+\sqrt{2}\left|\varepsilon_{3}^{\prime}\right| \frac{1}{\sqrt{2}}+\left|\varepsilon_{4}^{\prime}\right|\right) d_{i} d_{k} \leq 25.5 d_{i} d_{k} \varepsilon \\
& \left|\delta H_{j k}\right| \leq\left(\sqrt{2} \sqrt{2}\left|\varepsilon_{1}^{\prime}\right|+\left|\varepsilon_{2}^{\prime}\right|+2\left|\varepsilon_{3}^{\prime}\right|+\sqrt{2} \sqrt{2}\left|\varepsilon_{4}^{\prime}\right|\right) d_{j} d_{k} \leq 57 d_{j} d_{k} \varepsilon .
\end{aligned}
$$


Now we construct the 2 by 2 submatrix $\delta \widehat{H}_{m}$. The analysis is similar to the analysis in the trigonometric case because $a^{\prime}$ can be computed with small relative error. We have

$$
a^{\prime}=f l(a+c t)=\left(1+\varepsilon_{a^{\prime}}\right)\left(\left(1+\varepsilon_{a}\right) a+\widetilde{c} \widetilde{t}\right)
$$

where

$$
\left|\varepsilon_{a^{\prime}}\right| \leq 13 \varepsilon, \quad\left|\varepsilon_{a}\right| \leq 14 \varepsilon .
$$

Since $|t| \leq \beta$ and $|c|<\beta a$, we can write (with the relative error of $O(\varepsilon)$, of course)

$$
\left(1+\varepsilon_{a}\right) a+\widetilde{c} \tilde{t}=\left(1+\varepsilon_{a}^{\prime}\right)(a+\widetilde{c} \tilde{t})
$$

where

$$
\left|\varepsilon_{a}^{\prime}\right|=\left|\varepsilon_{a} \frac{\tilde{c} \tilde{t}}{a+\tilde{c} \tilde{t}}\right| \leq\left|\varepsilon_{a}\right| \frac{a \beta^{2}}{a\left(1-\beta^{2}\right)}=\left|\varepsilon_{a}\right| .
$$

Therefore,

$$
a^{\prime}=\left(1+\varepsilon_{a^{\prime}}^{\prime}\right)(a+\widetilde{c} \widetilde{t}), \quad\left|\varepsilon_{a^{\prime}}^{\prime}\right| \leq\left|\varepsilon_{a^{\prime}}\right|+\left|\varepsilon_{a}^{\prime}\right| \leq 27 \cdot \varepsilon
$$

Also

$$
b^{\prime}=f l(b+c t)=\left(1+\varepsilon_{b^{\prime}}\right)\left(b+\tilde{c} \tilde{t}+\varepsilon_{b} b\right), \quad\left|\varepsilon_{b^{\prime}}\right| \leq 13 \varepsilon,\left|\varepsilon_{b}\right| \leq 14 \varepsilon .
$$

Let

$$
\begin{aligned}
& \Delta_{1}=\left[\begin{array}{cc}
0 & \varepsilon_{c} c \\
\varepsilon_{c} c & 0
\end{array}\right]+J_{m}^{-1}\left[\begin{array}{cc}
0 & 0 \\
0 & \varepsilon_{b} b
\end{array}\right] J_{m}^{-1}, \\
& \Delta_{2}=\varepsilon_{a^{\prime}}^{\prime}\left(\left[\begin{array}{ll}
a & c \\
c & b
\end{array}\right]+\Delta_{1}\right), \\
& \Delta_{3}=J_{m}^{-1}\left[\begin{array}{cc}
0 & 0 \\
0 & b^{\prime}\left(1-\frac{1+\varepsilon_{a^{\prime}}^{\prime}}{1+\varepsilon_{b^{\prime}}}\right)
\end{array}\right] J_{m}^{-1}=\left[\begin{array}{cc}
\widetilde{s h}^{2} \varepsilon_{b^{\prime \prime}}^{\prime} b^{\prime} & -\widetilde{c h} \widetilde{s h} \varepsilon_{b^{\prime \prime}}^{\prime \prime} b^{\prime} \\
-\widetilde{c h} \widetilde{s h} \varepsilon_{b^{\prime \prime}}^{\prime} b^{\prime} & \widetilde{c h} \varepsilon_{b^{\prime \prime}}^{2} b^{\prime}
\end{array}\right],
\end{aligned}
$$

where $\left|\varepsilon_{b^{\prime \prime}}^{\prime}\right| \leq\left|\varepsilon_{a^{\prime}}^{\prime}\right|+\left|\varepsilon_{b^{\prime}}\right| \leq 40 \varepsilon$. Then

$$
J_{m}\left(\left[\begin{array}{cc}
a & c \\
c & b
\end{array}\right]+\Delta_{1}+\Delta_{2}+\Delta_{3}\right) J_{m}=\left[\begin{array}{cc}
a^{\prime} & 0 \\
0 & b^{\prime}
\end{array}\right],
$$

and

$$
\left\|\delta \widehat{A}_{m}\right\|_{2} \leq\left|\varepsilon_{c}\right|+3\left|\varepsilon_{b}\right|+2\left|\varepsilon_{a^{\prime}}^{\prime}\right|+3\left|\varepsilon_{b^{\prime \prime}}^{\prime}\right| \leq 225.5 \varepsilon .
$$

Combining (3.2.26) with the above relation, we finally obtain

$$
\left\|\delta A_{m}\right\|_{2} \leq(225.5+62.45 \sqrt{n-2}) \varepsilon .
$$

The theorem now follows from the relations (3.2.10), (3.2.22), (3.2.24) and (3.2.27). Q.E.D. 
Corollary 3.2.2 Assume Algorithm 3.1.1 converges, and that $H_{M}, J$ is the final pair. Write $H_{m}=D_{m} A_{m} D_{m}$ with $D_{m}$ diagonal and $A_{m}$ with ones on the diagonal for $0 \leq$ $m \leq M$. Let $\lambda_{j}$ be the $j$-th eigenvalue of the pair $H, J \equiv H_{0}, J$ and $\lambda_{j}^{\prime}=\left(H_{M}\right)_{j j} J_{j j}$. Then, with the relative error of $O(\varepsilon)$, the following error bound holds:

$$
\frac{\left|\lambda_{j}-\lambda_{j}^{\prime}\right|}{\left|\lambda_{j}\right|} \leq \varepsilon \sum_{m=0}^{M-1} \frac{C_{m}}{\lambda_{\min }\left(A_{m}\right)}+n \cdot \text { tol } .
$$

Proof. For every vector $x$ and positive definite $H$ we have

$$
\begin{aligned}
\left|x^{*} \delta H x\right| & \leq\left|x^{*} D \delta A D x\right| \leq\|\delta A\|_{2}\left|x^{*} D D x\right| \leq\|\delta A\|_{2} \frac{\left|x^{*} D A D x\right|}{\lambda_{\min }(A)} \\
& =\frac{\|\delta A\|_{2}}{\lambda_{\min }(A)} x^{*} H x .
\end{aligned}
$$

Let $\lambda_{m, j}$ denote the $j$-th eigenvalue of the pair $H_{m}, J$. Applying Th. 2.2.1 with $\delta J=0$ and $\eta_{J}=0$, and Th. 3.2.1 to the pairs $J, H_{m}$ for $0 \leq m \leq M-1$, we obtain

$$
1-\eta_{m} \leq \frac{\lambda_{m+1, j}}{\lambda_{m, j}} \leq 1+\eta_{m}
$$

where

$$
\eta_{m}=\frac{C_{m}}{\lambda_{\min }\left(A_{m}\right)} \varepsilon
$$

Applying Th. 2.2.1 and the stopping criterion to the pair $J, H_{M}$, and ignoring the $O\left(t o l^{2}\right)$ term, we obtain

$$
1-n \cdot t o l \leq \frac{\lambda_{j}^{\prime}}{\lambda_{M, j}} \leq 1+n \cdot \text { tol }
$$

Here we also used the fact that $\lambda_{\min }\left(A_{M}\right) \geq 1-n \cdot$ tol. Since

$$
\frac{\lambda_{j}^{\prime}}{\lambda_{J}}=\frac{\lambda_{1, j}}{\lambda_{j}} \cdot \frac{\lambda_{2, j}}{\lambda_{1, j}} \cdots \frac{\lambda_{M, j}}{\lambda_{M-1, j}} \cdot \frac{\lambda_{j}^{\prime}}{\lambda_{M, j}},
$$

the corollary follows by inserting (3.2.29) and (3.2.30) in the above relation, and ignoring the relative error of $O(\varepsilon)$.

Q.E.D.

Here are some remarks about Th. 3.2.1 and Cor. 3.2.2. The remarks hold for all subsequent theorems and corollaries of the above type.

Remark 3.2.3 In the hyperbolic case for $\zeta \leq \alpha=3 / 2 \sqrt{2}$ (Case I), the constant $C_{m}$ depends additionally on $\sqrt{\kappa\left(A_{m}\right)}$. Deichmöller [8] also obtained a similar bound for some non-orthogonal transformations. 
Remark 3.2.4 In practical computation Case I of Th. 3.2.1 occurs rarely, and almost never if we transform the pair $H, I \equiv G J G^{T}, I$ to the pair $G^{T} G, J$ (due to diagonalizing effect of this transformation). Thus, our choice of $\alpha$ (and its function $\beta)$ in (3.2.17) implies that the discontinuity of the bound $(3.2 .2)$ at $|\zeta|=3 /(2 \sqrt{2})$ has little practical importance. This discontinuity can be removed by considering Case I, $|\zeta| \leq 3 /(2 \sqrt{2})$, as Case II, $|\zeta|>\alpha^{\prime}$, for some $\alpha^{\prime}<3 /(2 \sqrt{2})$. Also note that $\beta$ cannot have an optimizing function as $\bar{x}$ in the trigonometric case, where the choice of $\bar{x}$ makes the bounds in the relations (3.2.7) and (3.2.9) almost equal. We can choose another approach when analysing the hyperbolic case in Th. 3.2.1, namely to analyse only the cases $d_{j} / d_{i} \geq \beta$ and $d_{j} / d_{i}<\beta$. Then the bounds (3.2.22) and (3.2.27) hold in the first and the second case, respectively. The approach of Th. 3.2.1 is, however, more enlightening and it simplifies the analysis of the modified method in the following subsection.

Remark 3.2.5 The $\sqrt{n-2}$ part of $C_{m}$ may be multiplied by $\max _{m, i \neq j}\left|A_{m, i j}\right|<1$. Thus if the matrices $A_{m}$ are strongly diagonally dominant, the part of the error term which depends on $n$ is suppressed.

Remark 3.2.6 Numerical experiments indicate that (3.2.28) grows only slowly with the increase of $n$ or $M$.

$æ$ 


\subsubsection{The modified method}

In order to avoid potentially large $C_{m}$ in Th. 3.2.1 in the hyperbolic case for $|\zeta| \leq \alpha=$ $3 /(2 \sqrt{2})$, we modify the $J$-orthogonal Jacobi method by bounding the hyperbolic angle as suggested in [29]. Since the original method converges, large hyperbolic angles can occur only finitely many times. We first show that the modification does not affect convergence properties. We then prove that one step of the modified method satisfies the assumptions needed for the error bounds of Chap. 2, i.e. that Th. 3.2.1 and Cor. 3.2.2 hold with small modifications. The algorithm of the modified $J$-orthogonal Jacobi method is similar to Alg. 3.1.1. The only changes are the computation of the hyperbolic rotation parameters and the update of the pivot submatrix $\widehat{H}$.

Algorithm 3.2.7 Modified two-sided J-orthogonal Jacobi method for the problem (3.1.1).

${ }^{*}$ compute the parameter hyp: hyp $=1$ for the hyperbolic and hyp $=-1$ for the trigonometric rotation, respectively */

if $1 \leq i \leq$ npos $<j \leq n$ then

$$
\text { hyp }=1
$$

else

$$
\text { hyp }=-1
$$

endif

${ }^{*}$ compute the hyperbolic Jacobi rotation which diagonalizes

$$
\begin{aligned}
& {\left[\begin{array}{ll}
H_{i i} & H_{i j} \\
H_{j i} & H_{j j}
\end{array}\right] \equiv\left[\begin{array}{ll}
a & c \\
c & b
\end{array}\right], \text { and update the 2 by 2 pivot submatrix */ }} \\
& \zeta=-h y p *(b+h y p * a) /(2 c) \\
& \text { if hyp }=1 \text { and }|\zeta| \leq \alpha \equiv 3 /(2 \sqrt{2}) \text { then } \\
& \quad c s=\sqrt{2} \\
& \quad \text { sn }=\operatorname{sign}(\zeta) \\
& \quad \text { sn } 1=\operatorname{sn} \\
& \quad H_{i i}=2 * a+b-2 * \sqrt{2} *|c| \\
& \quad H_{j j}=a+2 * b-2 * \sqrt{2} *|c| \\
& \quad H_{i j}=H_{j i}=\operatorname{sn} * \sqrt{2} *(a+b)+3 * c \\
& \text { else } \\
& \quad t=\operatorname{sign}(\zeta) /\left(|\zeta|+\sqrt{\zeta^{2}-h y p}\right) \\
& \quad h=\sqrt{1-h y p * t^{2}} \\
& \quad c s=1 / h \\
& \text { sn }=t / h \\
& \text { sn } 1=h y p * s n \\
& H_{i i}=a+h y p * c * t \\
& H_{j j}=b+c * t \\
& H_{i j}=H_{j i}=0 \\
& \text { endif }
\end{aligned}
$$


proceed as in Algorithm 3.1.1

The convergence proof for the modified method [29] rests on the trace reduction which takes place in our case, too. Let $|\zeta| \leq \alpha$ and let $H^{\prime}$ and $H^{\prime \prime}$ denote the matrices after an unmodified and modified step, respectively. Then

$$
a^{\prime \prime}=2 a+b-2 \sqrt{2}|c|, \quad b^{\prime \prime}=a+2 b-2 \sqrt{2}|c|,
$$

and

$$
\begin{aligned}
\delta \operatorname{Tr}^{\prime} & \equiv \operatorname{Tr}(H)-\operatorname{Tr}\left(H^{\prime}\right)=2|c||t| \\
\delta \operatorname{Tr}^{\prime \prime} & \equiv \operatorname{Tr}(H)-\operatorname{Tr}\left(H^{\prime \prime}\right)=-2 a-2 b+4 \sqrt{2}|c|
\end{aligned}
$$

The quotient $\delta \operatorname{Tr}^{\prime \prime} / \delta \operatorname{Tr}^{\prime} \leq 1$ is bounded below with $\beta$. For $\alpha$ and $\beta$ from (3.2.17), we have

$$
\frac{\delta T r^{\prime \prime}}{\delta T r^{\prime}}=\frac{1}{|t|}(2 \sqrt{2}-2|\zeta|) \geq 2 \sqrt{2}-2 \alpha=\frac{1}{\sqrt{2}} .
$$

This trace reduction is quite acceptable. Also, modified steps do not affect the quadratic convergence. Indeed, Drmač and Hari [15] showed that the hyperbolic tangent is bounded by $|t| \leq \sqrt{2} / 6$ after the quadratic convergence starts. This, in turn, implies $|\zeta| \geq 3 / \sqrt{2}$, so the modified steps do not occur after the quadratic convergence starts.

The next theorem is an analog of Th. 3.2.1 and Cor. 3.2.2 for the modified $J$-orthogonal Jacobi method.

Theorem 3.2.8 Let $H_{m}$ be the sequence of matrices generated by Algorithm 3.2.7 in floating-point arithmetic with precision $\varepsilon$. Then Theorem 3.2.1 holds except that in the hyperbolic case for $|\zeta| \leq 3 /(2 \sqrt{2})$ the value of $C_{m}$ is reduced to

$$
C_{m}=82+19.63 \sqrt{n-2} .
$$

Corollary 3.2.2 holds with this exception, too.

Proof. The technique of the proof is the same as in Th. 3.2.1. We assume without loss of generality that $s h=+1$. Then sign $(c)=-1$. Using (3.2.1) we obtain

$$
\begin{aligned}
& H_{i k}^{\prime \prime}=f l\left(\sqrt{2} H_{i k}+H_{j k}\right)=\sqrt{2} H_{i k}+H_{j k}+\varepsilon_{1}^{\prime} H_{i k}+\varepsilon_{1} H_{j k} \\
& H_{j k}^{\prime \prime}=f l\left(\sqrt{2} H_{j k}+H_{i k}\right)=\sqrt{2} H_{j k}+H_{i k}+\varepsilon_{2}^{\prime} H_{j k}+\varepsilon_{2} H_{i k},
\end{aligned}
$$

where $\left|\varepsilon_{1}^{\prime}\right|,\left|\varepsilon_{2}^{\prime}\right| \leq 3 \sqrt{2} \varepsilon$. Since $d_{j} / d_{i} \geq \beta$, we have

$$
\left|\delta H_{i k}\right| \leq(7+4 \sqrt{2}) d_{i} d_{k} \varepsilon, \quad\left|\delta H_{j k}\right| \leq 15 d_{j} d_{k} \varepsilon .
$$


Further,

$$
\begin{aligned}
& a^{\prime \prime}=f l(2 a+b-2 \sqrt{2}|c|)=2 a+b-2 \sqrt{2}|c|+\varepsilon_{3}^{\prime} a+\varepsilon_{3} b+\varepsilon_{4}^{\prime}|c| \\
& b^{\prime \prime}=f l(a+2 b-2 \sqrt{2}|c|)=a+2 b-2 \sqrt{2}|c|+\varepsilon_{4} a+\varepsilon_{5}^{\prime} b+\varepsilon_{6}^{\prime}|c| \\
& c^{\prime \prime}=f l(\sqrt{2}(a+b)+3 c)=\sqrt{2}(a+b)+3 c+\varepsilon_{7}^{\prime}(a+b)+\varepsilon_{8}^{\prime} c
\end{aligned}
$$

where

$$
\left|\varepsilon_{3}^{\prime}\right|,\left|\varepsilon_{5}^{\prime}\right| \leq 4 \varepsilon, \quad\left|\varepsilon_{4}^{\prime}\right|,\left|\varepsilon_{6}^{\prime}\right| \leq 8 \sqrt{2} \varepsilon, \quad\left|\varepsilon_{7}^{\prime}\right| \leq 4 \sqrt{2} \varepsilon, \quad\left|\varepsilon_{8}^{\prime}\right| \leq 6 \varepsilon
$$

Setting

$$
\Delta=\left[\begin{array}{cc}
\varepsilon_{3}^{\prime} a+\varepsilon_{3} b+\varepsilon_{4}^{\prime}|c| & \varepsilon_{7}^{\prime}(a+b)+\varepsilon_{8}^{\prime} c \\
\varepsilon_{7}^{\prime}(a+b)+\varepsilon_{8}^{\prime} c & \varepsilon_{4} a+\varepsilon_{5}^{\prime} b+\varepsilon_{6}^{\prime}|c|
\end{array}\right]
$$

we have

$$
J_{m}\left(\left[\begin{array}{ll}
a & c \\
c & b
\end{array}\right]+J_{m}^{-1} \Delta J_{m}^{-1}\right) J_{m}=\left[\begin{array}{cc}
a^{\prime \prime} & c^{\prime \prime} \\
c^{\prime \prime} & b^{\prime \prime}
\end{array}\right] .
$$

Using $d_{j} / d_{i} \geq \beta$, we obtain

$$
\left\|\delta \widehat{A}_{m}\right\|_{2} \leq 82 \varepsilon
$$

and finally (3.2.32).

Q.E.D.

We have thus eliminated $\kappa\left(A_{m}\right)$ from $C_{m}$ in the hyperbolic case for $\zeta \leq 3 /(2 \sqrt{2})$. This makes the one-step error bounds for the modified method of the same type as the corresponding bounds from [13], that is, the bounds depend only on $\sqrt{n-2}$. For $2 \times 2$ matrices, the use of modified rotations makes obviously no improvement. For $n \geq 3$, however, numerical experiments show that the use of modified rotations generally does not affect the convergence. Thus, the use of modified rotations generally decreases relative error estimates.

$æ$ 


\subsubsection{Growth of the condition of the scaled matrix}

As we have seen in Cor. 3.2.2, the behaviour of the quotient $\lambda_{\min }\left(A_{0}\right) / \lambda_{\min }\left(A_{m}\right)$ (or $\kappa\left(A_{m}\right) / \kappa\left(A_{0}\right)$ ) is essential for the overall error bound of the $J$-orthogonal Jacobi method. In this subsection we first state known results. We then show that $\kappa\left(A_{m}\right) / \kappa\left(A_{0}\right) \leq n$ if $\kappa(A) \geq \kappa(H)$. After that we give a simple pattern for the behaviour of the upper bound for $\lambda_{\min }\left(A_{0}\right) / \lambda_{\min }\left(A_{m}\right)$. As a corollary we show that, with the appropriate choice of pivots, we can perform $n^{\prime} \leq n-1$ successive steps such that $\lambda_{\min }\left(A_{0}\right) / \lambda_{\min }\left(A_{m}\right) \leq n$ for every $1 \leq m \leq n^{\prime}$. In the conclusion, we define an algorithm for calculating the upper bound for $\lambda_{\min }\left(A_{0}\right) / \lambda_{\min }\left(A_{m}\right)$ in Jacobi process. Results of numerical experiments are given in Chap. 5 . The results of this subsection are partially contained in [26].

We now state the known bounds for $1 / \lambda_{\min }\left(A_{m}\right)$, which were originally proved for the case $n$ pos $=n$ by Demmel and Veselić [13]. Later Veselić [28] noticed that the results also hold if the hyperbolic rotations are used, since the proofs do not require the orthogonality of rotation matrices. Let the pair $H_{m}, J$ be obtained from the pair $H_{0}, J$ by applying $m$ Jacobi rotations in pairwise nonoverlapping rows and columns (this means $m \leq n / 2)$, and let $\left(i_{k}, j_{k}\right)$ be the pivot pair in the $k$-th step. We use the standard scaling, i.e.

$$
H_{m}=D_{m} A_{m} D_{m}
$$

where $D_{m}$ is positive definite diagonal matrix, and $A_{m}$ has ones on the diagonal. The spectrum of $A_{m}$ coincides with the spectrum of the pencil $A_{0}-\lambda A_{0}^{\prime}$, where $A_{0}^{\prime}$ coincides with $A_{0}$ on every rotated element and is the identity otherwise. This implies

$$
\frac{1}{\lambda_{\min }\left(A_{m}\right)}=\max _{x \neq 0} \frac{x^{T} A_{0}^{\prime} x}{x^{T} A_{0} x} \leq \frac{\max _{x \neq 0,\|x\|_{2}=1} x^{T} A_{0}^{\prime} x}{\min _{x \neq 0,\|x\|_{2}=1} x^{T} A_{0} x}=\frac{1+\max _{0 \leq k \leq m-1}\left|A_{0, i_{k} j_{k}}\right|}{\lambda_{\min }\left(A_{0}\right)} .
$$

After $m$ arbitrary steps we have

$$
\frac{1}{\lambda_{\min }\left(A_{m}\right)} \leq \frac{\prod_{k=0}^{m-1}\left(1+\left|A_{k, i_{k} j_{k}}\right|\right)}{\lambda_{\min }\left(A_{0}\right)}
$$

The above upper bound for $1 / \lambda_{\min }\left(A_{m}\right)$ is usually a large overestimate.

The second bound is based on the Hadamard measure of a symmetric positive definite matrix $H$,

$$
\mathcal{H}(H) \equiv \frac{\operatorname{det}(H)}{\prod_{i} H_{i i}}
$$

It is easy to see that $\mathcal{H}(H) \leq 1$ and $\mathcal{H}(H)=1$ if and only if $H$ is diagonal. $\mathcal{H}(H)$ is independant of the scaling so that

$$
\mathcal{H}(H)=\mathcal{H}(A)=\operatorname{det} A .
$$

Furthermore,

$$
\frac{1}{\lambda_{\min }\left(A_{m}\right)} \leq \frac{e}{\mathcal{H}\left(H_{m}\right)}
$$


where $e=\exp (1)$, and

$$
\frac{1}{\mathcal{H}\left(H_{m+1}\right)}=\frac{1-A_{m, i j}^{2}}{\mathcal{H}\left(H_{m}\right)} \leq \frac{1}{\mathcal{H}\left(H_{m}\right)},
$$

where $(i, j)$ is the pivot pair in the $m$-th step. The above two relations can be used to monitor the convergence of $1 / \lambda_{\min }\left(A_{m}\right)$ to 1 , but they can be a large overestimate in the beginning of the diagonalization process. Finally, (3.2.35) and (3.2.36) give the guaranteed upper bound

$$
\max _{m} \frac{1}{\lambda_{\min }\left(A_{m}\right)} \leq \frac{e}{\operatorname{det}\left(A_{0}\right)}=\frac{e}{\mathcal{H}\left(H_{0}\right)} .
$$

The following simple result seems not to have attracted attention:

Proposition 3.2.9 Let npos $=n$ and $\kappa(A) \geq \kappa(H)$. Let $H_{m}, J$ be the sequence of pairs obtained by the J-orthogonal Jacobi method from the starting pair $H, J$. Then

$$
\kappa\left(A_{m}\right) / \kappa\left(A_{0}\right) \leq n
$$

where matrices $A_{m}$ are defined by (3.2.33).

Proof. The assumption npos $=n$ implies that all rotation matrices are orthogonal. The assumption $\kappa(A) \geq \kappa(H)$ and (1.5) imply

$$
\kappa\left(A_{m}\right) \leq n \min _{D} \kappa\left(D A_{m} D\right) \leq n \kappa\left(H_{m}\right)=n \kappa(H) \leq n \kappa(A) .
$$

Q.E.D.

Now we come to the central result of this subsection:

Theorem 3.2.10 Let $H_{m}=D_{m} A_{m} D_{m}$ be the sequence of matrices obtained by Algorithm 3.1.1 from the starting matrix $H \equiv H_{0}$, i.e.

$$
H_{m}=J_{m-1}^{T} H_{m-1} J_{m-1} .
$$

Let us define the sequence of matrices $T_{m}$ by

$$
\begin{aligned}
T_{0} & =I \\
T_{m} & =T_{m-1} U_{m} \\
U_{m} & =D_{m-1}^{-1} J_{m-1}^{-T} D_{m} .
\end{aligned}
$$

Then for $m \geq 1$

$$
\begin{aligned}
A_{m} & =T_{m}^{-1} A_{0} T_{m}^{-T} \\
\frac{1}{\lambda_{\min }\left(A_{m}\right)} & \equiv\left\|A_{m}^{-1}\right\|_{2} \leq\left\|A_{0}^{-1}\right\|_{2}\left\|T_{m}\right\|_{2}^{2} \leq\left\|A_{0}^{-1}\right\|_{2}\left\|T_{m}\right\|_{E}^{2}=\frac{\left\|T_{m}\right\|_{E}^{2}}{\lambda_{\min }\left(A_{0}\right)}
\end{aligned}
$$


and

$$
\left\|T_{m}\right\|_{E}^{2}=\left\|T_{m-1}\right\|_{E}^{2}+2 A_{m-1, i j} T_{m-1, i}^{T} T_{m-1, \cdot j} .
$$

Here $(i, j)$ is the pivot pair in the $m$-th step, and $T_{m-1, i}$ denotes the $i$-th column of $T_{m-1}$, etc.

Proof. The first two statements of the theorem are obvious. Moreover, since $A_{m} \rightarrow I$ as $m \rightarrow \infty$, the relation

$$
T_{m} A_{m} T_{m}^{T}=A_{0}
$$

implies

$$
\lim _{m \rightarrow \infty} T_{m} T_{m}^{T}=A_{0}
$$

and

$$
\lim _{k \rightarrow \infty} U_{m+1} \cdots U_{k} U_{k}^{T} \cdots U_{m+1}^{T}=A_{m} .
$$

It remains to prove the relation (3.2.38). From the definition of $U_{m}$ we see that only its pivot submatrix $\widehat{U}_{m}$ differs from the identity matrix, and that

$$
\widehat{U}_{m} \widehat{U}_{m}^{T}=\widehat{A}_{m-1} .
$$

Also, $U_{m}^{-1} A_{m-1} U_{m}^{-T}=A_{m}$. Now we show that

$$
U_{m}=\bar{J}_{m}^{-T} \bar{D}_{m} R_{m}
$$

where $\bar{J}_{m}$ is a $J$-orthogonal Jacobi rotation on $A_{m-1}, \bar{D}_{m}^{-1}$ scales $\bar{J}_{m}^{T} A_{m-1} \bar{J}_{m}$, and $R_{m}$ is orthogonal. Indeed, set

$$
\widehat{R}_{m}=\widehat{\bar{D}}_{m} \widehat{\bar{J}}_{m}^{-1} \widehat{D}_{m-1} \widehat{J}_{m-1} \widehat{D}_{m}^{-1}
$$

Then (3.2.39) is satisfied and $R_{m}$ is orthogonal since

$$
\begin{aligned}
\widehat{R}_{m}^{T} \widehat{R}_{m} & =\widehat{D}_{m}^{-1} \widehat{J}_{m-1}^{T} \widehat{D}_{m-1} \widehat{\bar{J}}_{m}^{-T} \widehat{\bar{D}}_{m}^{2} \widehat{\bar{J}}_{m}^{-1} \widehat{D}_{m-1} \widehat{J}_{m-1} \widehat{D}_{m}^{-1} \\
& =\widehat{D}_{m}^{-1} \widehat{J}_{m-1}^{T} \widehat{D}_{m-1} \widehat{A}_{m-1} \widehat{D}_{m-1} \widehat{J}_{m-1} \widehat{D}_{m}^{-1} \\
& =\widehat{D}_{m}^{-1} \widehat{J}_{m-1}^{T} \widehat{H}_{m-1} \widehat{J}_{m-1} \widehat{D}_{m}^{-1} \\
& =\widehat{D}_{m}^{-1} \widehat{H}_{m} \widehat{D}_{m}^{-1}=\widehat{I}
\end{aligned}
$$

Note that the above relation holds for trigonometric as well as for hyperbolic rotations.

In the multiplication

$$
T_{m}=T_{m-1} U_{m}
$$

only the $i$-th and $j$-th column of $T_{m-1}$ change, i.e.

$$
\left[\begin{array}{ll}
T_{m, \cdot i} & T_{m, \cdot j}
\end{array}\right]=\left[\begin{array}{ll}
T_{m-1, \cdot i} & T_{m-1, \cdot j}
\end{array}\right] \widehat{U}_{m} .
$$


Let $a \equiv A_{m-1, i_{m} j_{m}}$ and

$$
\widehat{R}_{m}=\left[\begin{array}{cc}
c & s \\
-s & c
\end{array}\right]
$$

In the trigonometric case we have

$$
\widehat{\bar{J}}_{m}=\frac{1}{\sqrt{2}}\left[\begin{array}{cc}
1 & 1 \\
-1 & 1
\end{array}\right], \quad \widehat{\bar{D}}_{m}=\left[\begin{array}{cc}
\sqrt{1-a} & 0 \\
0 & \sqrt{1+a}
\end{array}\right] .
$$

which, together with (3.2.39) and (3.2.40), implies

$$
\begin{aligned}
{\left[\begin{array}{ll}
T_{m, k i} & T_{m, k j}
\end{array}\right]=} & {\left[\begin{array}{ll}
T_{m-1, k i} & T_{m-1, k j}
\end{array}\right] . } \\
& \cdot \frac{1}{\sqrt{2}}\left[\begin{array}{cc}
c \sqrt{1-a}-s \sqrt{1+a} & s \sqrt{1-a}+c \sqrt{1+a} \\
-c \sqrt{1-a}-s \sqrt{1+a} & -s \sqrt{1-a}+c \sqrt{1+a}
\end{array}\right] .
\end{aligned}
$$

After a simple but rather long calculation, we obtain

$$
T_{m, k i}^{2}+T_{m, k j}^{2}=T_{m-1, k i}^{2}+T_{m-1, k j}^{2}+2 a T_{m-1, k i} T_{m-1, k j} .
$$

The relation (3.2.38) now follows by summing up (3.2.41) for $k=1, \ldots, n$.

In the hyperbolic case we have

$$
\widehat{\bar{J}}_{m}=\left[\begin{array}{cc}
c h & s h \\
s h & c h
\end{array}\right], \quad \widehat{\bar{D}}_{m}=\left[\begin{array}{cc}
\sqrt{1+t a} & 0 \\
0 & \sqrt{1+t a}
\end{array}\right]
$$

where (see Alg. 3.1.1)

$$
\begin{array}{r}
\zeta=-\frac{1}{a}, \quad t=-\frac{a}{1+\sqrt{1-a^{2}}}, \\
c h^{2}+s h^{2}=\frac{1}{\sqrt{1-a^{2}}}, \quad s h \cdot c h=-\frac{a}{2 \sqrt{1-a^{2}}} .
\end{array}
$$

This, together with (3.2.39) and (3.2.40), implies

$$
\left[\begin{array}{ll}
T_{m, k i} & T_{m, k j}
\end{array}\right]=\left[\begin{array}{ll}
T_{m-1, k i} & T_{m-1, k j}
\end{array}\right] \sqrt{1+t a}\left[\begin{array}{cc}
c \cdot c h+s \cdot s h & s \cdot c h-c \cdot s h \\
-c \cdot s h-s \cdot c h & -s \cdot s h+c \cdot c h
\end{array}\right] .
$$

After a simple but rather long calculation, we obtain again (3.2.41) and the theorem is proved.

Q.E.D.

Corollary 3.2.11 Let the matrices $H_{m}=D_{m} A_{m} D_{m}, T_{m}$, and $U_{m}$ be defined as in Theorem 3.2.10. Let us perform $n^{\prime} \leq n-1$ successive steps of the $J$-orthogonal Jacobi method such that for every $m \in\left\{1, \ldots, n^{\prime}\right\}$ and every $k \in\{1, \ldots, n\}$ either $T_{m-1, k i_{m}}=0$ or $T_{m-1, k j_{m}}=0$. Here $\left(i_{m}, j_{m}\right)$ denotes the pivot pair in the $m-$ th step. 
(These assumptions are fulfilled e.g. if we choose pivot pairs along the first row, or along the last column, or along the first off-diagonal.) Then

$$
1 / \lambda_{\min }\left(A_{m}\right) \leq n / \lambda_{\min }\left(A_{0}\right)
$$

for every $m \in\left\{1, \ldots, n^{\prime}\right\}$.

Proof. By definition is $\left\|T_{0}\right\|_{E}^{2}=n$. The corollary follows from the assumptions and the relations (3.2.38) and (3.2.37).

Q.E.D.

Now we derive an efficient algorithm for calculating the upper bound for $1 / \lambda_{\min }\left(A_{m}\right)$ in Jacobi process. The inequality (3.2.37) implies that

$$
1 / \lambda_{\min }\left(A_{m}\right) \leq\left\|T_{m}\right\|_{E}^{2} / \lambda_{\min }\left(A_{0}\right)
$$

We can calculate $\left\|T_{m}\right\|_{E}^{2}$ using the recursive equation (3.2.38) in the following manner: instead of keeping the eigenvector matrix $V$ according to Alg. 3.1.1,

$$
\begin{aligned}
V_{0} & =I \\
V_{m} & =J_{0} J_{1} \cdots J_{m-1}=V_{m-1} J_{m-1},
\end{aligned}
$$

we keep the matrix $S$ defined by

$$
\begin{aligned}
S_{0} & =D_{0}^{-1} \\
S_{m} & =D_{0}^{-1} J_{0}^{-T} J_{1}^{-T} \cdots J_{m-1}^{-T}=S_{m-1} J_{m-1}^{-T} .
\end{aligned}
$$

In the trigonometric case we have $J_{m-1}^{-T}=J_{m}$, and in the hyperbolic case we have

$$
\widehat{J}_{m-1}^{-T}=\widehat{J}_{m-1}^{-1}=\left[\begin{array}{cc}
c h & -s h \\
-s h & c h
\end{array}\right] \text {. }
$$

Also

$$
V_{m}^{-T}=D_{0} S_{m}, \quad T_{m}=S_{m} D_{m} .
$$

In order to apply (3.2.38), we need to calculate the scalar product of the $i$-th and $j$-th column of $T_{m}$. From (3.2.42), we see that

$$
T_{m, \cdot i}^{T} T_{m, \cdot j}=S_{m, i}^{T} S_{m, \cdot j} D_{m, i i} D_{m, j j}
$$

Therefore, the sequence $\left\|T_{m}\right\|_{E}^{2}$ is given by the recursion

$$
\begin{aligned}
\left\|T_{0}\right\|_{E}^{2} & =n \\
\left\|T_{m}\right\|_{E}^{2} & =\left\|T_{m-1}\right\|_{E}^{2}+2 H_{m-1, i j} S_{m-1, i}^{T} S_{m-1, \cdot j},
\end{aligned}
$$

at a cost of $n+2$ multiplications and $n$ additions in each step. 
Suppose that the algorithm converges, and that $H_{M}, J$ is the final pair. Then (3.2.42) implies that

$$
V_{M}^{-T}=D_{0} S_{M},
$$

but we want to obtain the eigenvector matrix $V_{M}$. Since $V_{M}$ is $J$-orthogonal, i.e. $V_{M}^{T} J V_{M}=J$, we have

$$
V_{M}=J V_{M}^{-T} J
$$

Multiplication with $D_{0}$ from left has relative error $\varepsilon$ and multiplications with $J$ have no error at all.

In numerical experiments sequence $\left\|T_{m}\right\|_{E}^{2}$ behaved extremely well in the sense that it was approximately $n$ for all $m$. However, the recursion (3.2.43) does not reveal the fact that $1 / \lambda_{\min }\left(A_{m}\right)$ tends to one. This convergence can be monitored using the monotonically decreasing upper bound (3.2.35). This bound is usually large in the beginning of the diagonalization process, and it meets the bound given by (3.2.43) after one or two cycles. After that point (3.2.43) is not needed any more. Updating $\mathcal{H}\left(H_{m}\right)$ according to (3.2.36) is very simple. The only additional effort is to calculate $\mathcal{H}\left(H_{0}\right)$ (for example by using the Cholesky decomposition of $H_{0}$ ).

Remark 3.2.12 The theoretical results of this section, as well as numerical observations, do not depend upon whether only trigonometric $(J=I)$, or trigonometric and hyperbolic rotations are used. This once more justifies the use of the hyperbolic rotations.

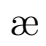




\subsection{Implicit $J$-orthogonal Jacobi method}

In this section we present and analyse the implicit (one-sided) $J$-orthogonal Jacobi method for solving the eigenvalue problem

$$
H x=\lambda x, \quad x \neq 0,
$$

where $H$ is a $n \times n$ real symmetric matrix of $\operatorname{rank} \operatorname{rank}(H)=r \leq n$. Let $H$ be decomposed as

$$
H=G J G^{T},
$$

where $G$ is a $n \times r$ matrix (i.e. $G$ has full column rank), $J=I_{\text {npos }} \oplus\left(-I_{r-n p o s}\right)$, and npos is number of the positive eigenvalues of $H$. The symmetric indefinite decomposition (3.3.2) is described in Chap. 4. Since $J^{-1}=J$, Th. 2.3.1 implies that the eigenvalues of the pair $G^{T} G, J$ are the nonzero eigenvalues of $H$, and that there exists a $J$-orthogonal matrix $F\left(F^{T} J F=J\right)$ such that the matrix

$$
F^{T} G^{T} G F \equiv \Delta
$$

is diagonal and positive definite. Therefore, nonzero eigenvalues of the problem (3.3.1) are the diagonal elements of the diagonal matrix $\Delta J$, and the corresponding eigenvectors are the columns of the matrix

$$
U=G F \Delta^{-1 / 2}
$$

Instead of forming explicitly the matrix $G^{T} G$ and applying Alg. 3.1.1 to the pair $G^{T} G, J$, we apply the implicit $J$-orthogonal Jacobi method to the pair $G, J$. The method, originally proposed by Veselić [29], consists of an iterative application of the one-sided transformation

$$
G_{m+1}=G_{m} J_{m}
$$

where $G \equiv G_{0}$ and $J_{m}$ is a $J$-orthogonal Jacobi plane rotation.

If $G$ is square and non-singular, the method also solves the hyperbolic singular value problem [21] for the pair $G, J$.

Note that in the positive definite case [13], the implicit method can be applied either to $G$ or $G^{T}$ (since $J=I$, the matrices $G^{T} G$ and $G G^{T}$ have the same eigenvalues and simply related eigenvectors). Here, even if $H$ is non-singular ( $G$ is non-singular and square), only one application makes sense, i.e. from the right on $G$ or from the left on $G^{T}$ (see also Sect. 2.3).

The section is organized as follows: we first present the algorithm. Then we prove that in floating-point arithmetic the method computes the non-zero eigenvalues of $H$ with the error bounds of Chap. 2. We analyse the simple version of the algorithm, omitting enhancements like keeping the diagonal in a separate vector and fast rotations, to make the error analysis clearer. In Subsect. 3.3.1 we analyse the version of the algorithm where diagonal of $G^{T} G$ is kept in a separate vector. In Subsect. 3.3.2 
we give the norm error bounds for the computed eigenvectors if $H$ is non-singular (non-singularity is neccessary since we use the eigenvector perturbation bounds from Chap. 2). In Sect. 3.4 we analyse the fast version of the algorithm. In Subsect. 3.4.1 we analyse the fast method which uses self-scaling rotations. These rotations, introduced and analysed by Anda and Park [1] for the trigonometric case, are used to suppress possible underflow/overflow when accumulating the diagonal of the fast rotations.

We now present our algorithm: 
Algorithm 3.3.1 Implicit J-orthogonal Jacobi method for the pair $G, J$. tol is a user defined stopping criterion.

repeat

for all pairs $i<j$

$$
\begin{aligned}
& /^{*} \text { compute }\left[\begin{array}{ll}
a & c \\
c & b
\end{array}\right] \equiv \text { the }(i, j) \text { submatrix of } G^{T} G * / \\
& \begin{array}{l}
a=\sum_{k=1}^{n} G_{k i}^{2} \\
b=\sum_{k=1}^{n} G_{k j}^{2} \\
c=\sum_{k=1}^{n} G_{k i} * G_{k j}
\end{array}
\end{aligned}
$$

${ }^{*}$ compute the parameter hyp: hyp $=1$ for the hyperbolic and

hyp $=-1$ for the trigonometric rotation, respectively */

if $1 \leq i \leq$ npos $<j \leq r$ then

$$
\text { hyp }=1
$$

else

$$
\text { hyp }=-1
$$

endif

${ }^{*}$ compute the $J$-orthogonal Jacobi rotation which diagonalizes

$$
\left[\begin{array}{ll}
H_{i i} & H_{i j} \\
H_{j i} & H_{j j}
\end{array}\right] \equiv\left[\begin{array}{cc}
a & c \\
c & b
\end{array}\right] * /
$$

$\zeta=-h y p *(b+h y p * a) /(2 c)$

$t=\operatorname{sign}(\zeta) /\left(|\zeta|+\sqrt{\zeta^{2}-h y p}\right)$

$h=\sqrt{1-h y p * t^{2}}$

$c s=1 / h$

$s n=t / h$

sn $1=h y p * s n$

${ }^{*}$ update columns $i$ and $j$ of $G * /$

for $k=1$ to $n$

$$
\begin{aligned}
& t m p=G_{k i} \\
& G_{k i}=c s * t m p+s n 1 * G_{k j} \\
& G_{k j}=s n * t m p+c s * G_{k j}
\end{aligned}
$$

endfor

endfor

until convergence $($ all $|c| / \sqrt{a b} \leq$ tol $)$

${ }^{*}$ the computed non-zero eigenvalues of $H=G J G^{T}$ (and of the pair $G^{T} G, J$ ) are

$$
\lambda_{j}=\left(\sum_{k=1}^{n} G_{k j}^{2}\right) J_{j j} * /
$$

${ }^{*}$ the computed eigenvectors of $H$ are the normalized columns of the final $G * /$

Remark 3.3.2 If $G$ is square and non-singular, then the computed hyperbolic singular values [21] of the pair $G, J$ are

$$
\sigma_{j}=\sqrt{\sum_{k=1}^{n} G_{k j}^{2}} J_{j j},
$$


and the computed hyperbolic singular vectors are the normalized columns of the final $G$. This remark holds for all subsequent implicit methods in this chapter.

The perturbation theory for the problem (3.3.1), as well as for the hyperbolic singular value problem [21], is given by Theorems 2.3.1 and 2.3.2. Let $G_{m}$ be the sequence of matrices obtained by Alg. 3.3.1 from the starting matrix $G \equiv G_{0}$. For every $m \geq 0$ write $G_{m}=B_{m} D_{m}$, where $D_{m}$ is diagonal positive definite, and the columns of $B_{m}$ have unit norms. All error bounds in this section contain the quantities $1 / \sigma_{\min }\left(B_{m}\right)$, whereas the perturbation bounds in Chap. 2 are proportional to $1 / \sigma_{\min }\left(B_{0}\right)$ (or $\left.\kappa\left(B_{0}\right)\right)$. Therefore, as in Sect. 3.2, our claim that the implicit $J$-orthogonal Jacobi method is as accurate as predicted in Sect. 2.3 depends on the ratio $\max _{m} \sigma_{\min }\left(B_{0}\right) / \sigma_{\min }\left(B_{m}\right)$ (or $\max _{m} \kappa\left(B_{m}\right) / \kappa\left(B_{0}\right)$ ) being modest. In exact arithmetic, one-sided Jacobi on $G=B D$ is identical to two-sided Jacobi on $H=G^{T} G=D B^{T} B D=D A D$. Thus, all convergence properties of the explicit method carry naturally over to the implicit one, and the question of the growth of $\kappa\left(B_{m}\right)=\kappa\left(A_{m}\right)^{1 / 2}$ is essentially identical to the question of the growth of $\kappa\left(A_{m}\right)$ in the case of two-sided Jacobi. Therefore, the results of Subsect. 3.2.2 apply here, as well.

The following theorem and its corollary justify our accuracy claims for the nonzero eigenvalues of the matrix $H=G J G^{T}$ computed by the implicit $J$-orthogonal Jacobi method.

Theorem 3.3.3 Let $G_{m}$ be the sequence of matrices generated by the implicit J-orthogonal Jacobi algorithm in floating-point arithmetic with precision $\varepsilon$; that is $G_{m+1}$ is obtained from $G_{m}$ by applying a single J-orthogonal Jacobi rotation. Then the following diagram commutes:

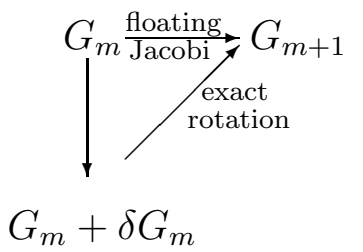

The top arrow indicates that $G_{m+1}$ is obtained from $G_{m}$ by applying one J-orthogonal Jacobi rotation in floating-point arithmetic. The diagonal arrow indicates that $G_{m+1}$ is obtained from $G_{m}+\delta G_{m}$ by applying one $J$-orthogonal plane rotation in exact arithmetic; thus $G_{m+1} J G_{m+1}^{T}$ and $\left(G_{m}+\delta G_{m}\right) J\left(G_{m}+\delta G_{m}\right)^{T}$ have identical non-zero eigenvalues and the corresponding eigenvectors. $\delta G_{m}$ is bounded as follows: let $\kappa=\kappa^{2}\left(B_{m}\right)$, and write $\delta G_{m}=\delta B_{m} D_{m}$, where $D_{m}$ is diagonal such that $B_{m}$ in $G_{m}=B_{m} D_{m}$ has unit columns. Let $a_{T}$ and $b_{T}$ be the true values of $\sum_{k} G_{k i}^{2}$ and $\sum_{k} G_{k j}^{2}$, respectively. Then, with the relative error of order $\varepsilon$,

$$
\left\|\delta B_{m}\right\|_{2} \leq C_{m} \varepsilon
$$


where

$$
C_{m}=\left\{\begin{array}{lll}
26 & \text { in trigonometric case , } & \\
\kappa+13 \sqrt{\kappa}+29 & \text { in hyperbolic case, } & |\zeta| \leq \frac{3}{2 \sqrt{2}}, \\
77 & \text { in hyperbolic case, } & |\zeta|>\frac{3}{2 \sqrt{2}}, \\
96 & \text { in hyperbolic case, } & |\zeta|>\frac{1}{2} a_{T}, \\
& & b_{T}<\frac{1}{2} a_{T} .
\end{array}\right.
$$

In other words, one step of the implicit J-orthogonal Jacobi method satisfies the assumptions needed for the perturbation bounds of Sect. 2.3.

Proof. The proof of the commuting diagram is a tedious computation. We shall prove the diagram separately for the trigonometric and for the hyperbolic case. Let $c_{T}$ be the true value of $\sum_{k} G_{k i} G_{k j}$. As in (3.2.3), we set

$$
a_{T}=d_{i}^{2}, \quad b_{T}=d_{j}^{2}, \quad c_{T}=z d_{i} d_{j} .
$$

We may assume without loss of generality that $a_{T} \geq b_{T}$ and $c_{T}>0$. As in (3.2.4), we have

$$
0<z \leq \bar{z} \equiv\left(\kappa^{2}\left(B_{m}\right)-1\right) /\left(\kappa^{2}\left(B_{m}\right)+1\right)<1 .
$$

Set $x \equiv d_{j} / d_{i}$. Note that $x \leq 1$. Systematic application of formulae (3.2.1) shows that

$$
\begin{array}{rrrr}
a=a_{T}\left(1+\varepsilon_{a}\right) & \text { where } & \left|\varepsilon_{a}\right| \leq n \varepsilon \\
b=b_{T}\left(1+\varepsilon_{b}\right) & \text { where } & \left|\varepsilon_{b}\right| \leq n \varepsilon \\
c=c_{T}+\varepsilon_{c} \sqrt{a_{T} b_{T}} & \text { where } & \left|\varepsilon_{c}\right| \leq n \varepsilon .
\end{array}
$$

Trigonometric case. This case was analysed by Demmel and Veselić [13] and we present it for the sake of completeness. Small differences in the proof give here, again, a somewhat better bound for $\left\|\delta B_{m}\right\|_{2}$.

Let

$$
\widetilde{c s} \equiv 1 / \sqrt{1+t^{2}}, \quad \widetilde{s n} \equiv t / \sqrt{1+t^{2}}
$$

From (3.2.1) we get

$$
s n=\left(1+\varepsilon_{s n}\right) \widetilde{s n}, \quad c s=\left(1+\varepsilon_{c s}\right) \widetilde{c s}, \quad\left|\varepsilon_{s n}\right|,\left|\varepsilon_{c s}\right| \leq 3 \varepsilon
$$

$\widetilde{c s}$ and $\widetilde{s n}$ define the exact rotation

$$
J_{m}=\left[\begin{array}{cc}
\widetilde{c s} & \widetilde{s n} \\
-\widetilde{s n} & \widetilde{c s}
\end{array}\right]
$$


which takes $G_{m}+\delta G_{m}$ to $G_{m+1}$ :

$$
\left(G_{m}+\delta G_{m}\right) J_{m}=G_{m+1} .
$$

Let $G_{k i}^{\prime}$ and $G_{k j}^{\prime}$ be the new values for these entries computed by the algorithm. Then

$$
\begin{aligned}
G_{k i}^{\prime} & =f l\left(c s * G_{k i}-s n * G_{k j}\right) \\
& =\left(1+\varepsilon_{1}\right)\left(1+\varepsilon_{2}\right) c s G_{k i}-\left(1+\varepsilon_{3}\right)\left(1+\varepsilon_{4}\right) s n G_{k j} \\
& =\left(1+\varepsilon_{1}\right)\left(1+\varepsilon_{2}\right)\left(1+\varepsilon_{c s}\right) \widetilde{c s} G_{k i}-\left(1+\varepsilon_{3}\right)\left(1+\varepsilon_{4}\right)\left(1+\varepsilon_{s n}\right) \widetilde{s n} G_{k j} \\
& \equiv \widetilde{c s} G_{k i}-\widetilde{s n} G_{k j}+E_{k i}
\end{aligned}
$$

and, similarly,

$$
G_{k j}^{\prime}=f l\left(s n * G_{k i}+c s * G_{k j}\right)=\widetilde{s n} G_{k i}+\widetilde{c s} G_{k j}+E_{k j}
$$

where

$$
\begin{aligned}
\left\|E_{\cdot i}\right\|_{2} & \leq 5\left(\widetilde{c s}\left\|G_{\cdot i}\right\|_{2}+|\widetilde{s n}|\left\|G_{\cdot j}\right\|_{2}\right) \varepsilon \\
\left\|E_{\cdot j}\right\|_{2} & \leq 5\left(|\widetilde{s n}|\left\|G_{\cdot i}\right\|_{2}+\widetilde{c s}\left\|G_{\cdot j}\right\|_{2}\right) \varepsilon .
\end{aligned}
$$

Here $G_{\text {.i }}$ refers to the $i$-th column of $G$, etc. Thus

$$
\begin{aligned}
{\left[\begin{array}{ll}
G_{\cdot i}^{\prime} & G_{\cdot j}^{\prime}
\end{array}\right] } & =\left[\begin{array}{ll}
G_{\cdot i} & G_{\cdot j}
\end{array}\right]\left[\begin{array}{cc}
\widetilde{c s} & \widetilde{s n} \\
-\widetilde{s n} & \widetilde{c s}
\end{array}\right]+\left[\begin{array}{cc}
E_{\cdot i} & E_{\cdot j}
\end{array}\right] \\
& =\left(\left[\begin{array}{ll}
G_{\cdot i} & G_{\cdot j}
\end{array}\right]+\left[\begin{array}{ll}
E_{\cdot i} & E_{\cdot j}
\end{array}\right]\left[\begin{array}{cc}
\widetilde{c s} & -\widetilde{s n} \\
\widetilde{s n} & \widetilde{c s}
\end{array}\right]\right)\left[\begin{array}{cc}
\widetilde{c s} & \widetilde{s n} \\
-\widetilde{s n} & \widetilde{c s}
\end{array}\right] \\
& \equiv\left(\left[\begin{array}{ll}
G_{\cdot i} & G_{\cdot j}
\end{array}\right]+\left[\begin{array}{ll}
F_{\cdot i} & F_{\cdot j}
\end{array}\right]\right)\left[\begin{array}{cc}
\widetilde{c s} & \widetilde{s n} \\
-\widetilde{s n} & \widetilde{c s}
\end{array}\right],
\end{aligned}
$$

where

$$
\begin{aligned}
\left\|F_{\cdot i}\right\|_{2} & \leq \widetilde{c s}\left\|E_{\cdot i}\right\|_{2}+|\widetilde{s n}|\left\|E_{\cdot j}\right\|_{2} \\
& \leq\left(5\left\|G_{\cdot i}\right\|_{2}+10 \widetilde{c s}|\widetilde{s n}|\left\|G_{\cdot j}\right\|_{2}\right) \varepsilon \\
& \leq 5(1+x) d_{i} \varepsilon
\end{aligned}
$$

and

$$
\begin{aligned}
\left\|F_{\cdot j}\right\|_{2} & \leq|\widetilde{s n}| \cdot\left\|E_{\cdot i}\right\|_{2}+\widetilde{c s}\left\|E_{\cdot j}\right\|_{2} \\
& \leq\left(5\left\|G_{\cdot j}\right\|_{2}+10 \widetilde{c s}|\widetilde{s n}|\left\|G_{\cdot i}\right\|_{2}\right) \varepsilon \\
& \leq 5(1+2 \widetilde{c s}|\widetilde{s n}| / x) d_{j} \varepsilon .
\end{aligned}
$$

We consider two cases, $x<\bar{x} \equiv 0.48$, and $x \geq \bar{x}$. First consider $x<\bar{x}$. By inserting $\bar{x}$ for $x$ in (3.3.8) we obtain

$$
\left\|F_{\cdot i}\right\|_{2} \leq 7.4 d_{i} \varepsilon
$$


Our assumption further implies that the subtraction $1-x^{2}$ has a low relative error, and that $z+n \varepsilon<1$ with a relative error of $O(\varepsilon)$. Therefore

$$
\begin{aligned}
|t| & \leq \frac{|c|}{|b-a|}=\frac{\left|c_{T}+\varepsilon_{c} \sqrt{a_{T} b_{T}}\right|}{\left|b_{T}+\varepsilon_{b} b_{T}-a_{T}-\varepsilon_{a} a_{T}\right|} \\
& =\frac{\left|z x+\varepsilon_{c} x\right|}{\left|x^{2}-1+\varepsilon_{b} x^{2}-\varepsilon_{a}\right|} \leq \frac{x(z+n \varepsilon)}{1-\bar{x}^{2}}(1+O(\varepsilon)) .
\end{aligned}
$$

We can ignore the $(z+n \varepsilon)(1+O(\varepsilon))$ term, so that $|t| \leq x /\left(1-\bar{x}^{2}\right)$. Inserting this inequality into (3.3.9) we obtain

$$
\left\|F_{\cdot j}\right\|_{2} \leq 5\left(1+\frac{2}{1-\bar{x}^{2}}\right) d_{j} \varepsilon \leq 18 d_{j} \varepsilon .
$$

Here we also used $\widetilde{c s}|\widetilde{s n}| \leq \widetilde{c s}^{2}|t| \leq|t|$. Therefore,

$$
\left\|\delta B_{m}\right\|_{2} \leq \frac{\left\|F_{\cdot i}\right\|_{2}}{d_{i}}+\frac{\left\|F_{\cdot j}\right\|_{2}}{d_{j}} \leq 26 \varepsilon
$$

Now consider the case $x \geq \bar{x}$. Inserting 1 for $x$ in (3.3.8) we obtain

$$
\left\|F_{. i}\right\|_{2} \leq 10 d_{i} \varepsilon
$$

Inserting $\widetilde{c s}|\widetilde{s n}| \leq 1 / 2$ and $1 / \bar{x}$ for $1 / x$ in (3.3.9), we obtain

$$
\left\|F_{\cdot j}\right\|_{2} \leq 15.5 d_{j} \varepsilon
$$

so that (3.3.11) holds again, thus improving the bound $\left\|\delta B_{m}\right\|_{2} \leq 72 \varepsilon$ from [13]. æ 
Hyperbolic case. For the sake of the clarity, we denote the quantities $c s$, sn and $s n 1=s n$ computed by Alg. 3.1.1 with $c h$ and $s h$, respectively. Let

$$
\widetilde{c h} \equiv 1 / \sqrt{1-t^{2}}, \quad \widetilde{s h} \equiv t / \sqrt{1-t^{2}} .
$$

Using (3.2.1) we can show that the bounds (3.2.11) hold for $t, \widetilde{c h}$ and $\widetilde{s h}$ with a relative error of $O(\varepsilon)$. Suppose that we can write

$$
s h=\left(1+\varepsilon_{s h}\right) \widetilde{s h}, \quad c h=\left(1+\varepsilon_{c h}\right) \widetilde{c h} .
$$

$\widetilde{c h}$ and $\widetilde{s h}$ define the exact rotation

$$
J_{m}=\left[\begin{array}{cc}
\widetilde{c h} & \widetilde{s h} \\
\widetilde{s h} & \widetilde{c h}
\end{array}\right]
$$

which takes $G_{m}+\delta G_{m}$ to $G_{m+1}$ :

$$
\left(G_{m}+\delta G_{m}\right) J_{m}=G_{m+1} .
$$

Let $G_{k i}^{\prime}$ and $G_{k j}^{\prime}$ be the new values for these entries computed by the algorithm. Then

$$
\begin{aligned}
G_{k i}^{\prime} & =f l\left(c h * G_{k i}+s h * G_{k j}\right) \\
& =\left(1+\varepsilon_{1}\right)\left(1+\varepsilon_{2}\right) \operatorname{ch} G_{k i}+\left(1+\varepsilon_{3}\right)\left(1+\varepsilon_{4}\right) s h G_{k j} \\
& =\left(1+\varepsilon_{1}\right)\left(1+\varepsilon_{2}\right)\left(1+\varepsilon_{c h}\right) \widetilde{c h} G_{k i}+\left(1+\varepsilon_{3}\right)\left(1+\varepsilon_{4}\right)\left(1+\varepsilon_{s h}\right) \widetilde{s h} G_{k j} \\
& \equiv \widetilde{c h} G_{k i}+\widetilde{s h} G_{k j}+E_{k i},
\end{aligned}
$$

and, similarly,

$$
G_{k j}^{\prime}=f l\left(s h * G_{k i}+c h * G_{k j}\right)=\widetilde{s h} G_{k i}+\widetilde{c h} G_{k j}+E_{k j},
$$

where

$$
\begin{aligned}
\left\|E_{\cdot i}\right\|_{2} & \leq\left|\varepsilon_{1}^{\prime}\right| \widetilde{c h}\left\|G_{\cdot i}\right\|_{2}+\left|\varepsilon_{2}^{\prime}\right||\widetilde{s h}|\left\|G_{\cdot j}\right\|_{2} \\
\left\|E_{\cdot j}\right\|_{2} & \leq\left|\varepsilon_{3}^{\prime}\right||\widetilde{s h}|\left\|G_{\cdot i}\right\|_{2}+\left|\varepsilon_{4}^{\prime}\right| \widetilde{c h}\left\|G_{\cdot j}\right\|_{2} .
\end{aligned}
$$

Here

$$
\left|\varepsilon_{1}^{\prime}\right|,\left|\varepsilon_{4}^{\prime}\right|=\left|\varepsilon_{c h}\right|+2 \varepsilon, \quad\left|\varepsilon_{2}^{\prime}\right|,\left|\varepsilon_{3}^{\prime}\right|=\left|\varepsilon_{s h}\right|+2 \varepsilon
$$

Thus

$$
\begin{aligned}
{\left[\begin{array}{ll}
G_{\cdot i}^{\prime} & G_{\cdot j}^{\prime}
\end{array}\right] } & =\left[\begin{array}{ll}
G_{\cdot i} & G_{\cdot j}
\end{array}\right]\left[\begin{array}{cc}
\widetilde{c h} & \widetilde{s h} \\
\widetilde{s h} & \widetilde{c h}
\end{array}\right]+\left[\begin{array}{ll}
E_{\cdot i} & E_{\cdot j}
\end{array}\right] \\
& =\left(\left[\begin{array}{ll}
G_{\cdot i} & G_{\cdot j}
\end{array}\right]+\left[\begin{array}{ll}
E_{\cdot i} & E_{\cdot j}
\end{array}\right]\left[\begin{array}{cc}
\widetilde{c h} & -\widetilde{s h} \\
-\widetilde{s h} & \widetilde{c h}
\end{array}\right]\right)\left[\begin{array}{cc}
\widetilde{c h} & \widetilde{s h} \\
\widetilde{s h} & \widetilde{c h}
\end{array}\right] \\
& \equiv\left(\left[\begin{array}{ll}
G_{\cdot i} & G_{\cdot j}
\end{array}\right]+\left[\begin{array}{ll}
F_{\cdot i} & F_{\cdot j}
\end{array}\right]\right)\left[\begin{array}{cc}
\widetilde{c h} & \widetilde{s h} \\
\widetilde{s h} & \widetilde{c h}
\end{array}\right]
\end{aligned}
$$


where

$$
\begin{aligned}
\left\|F_{\cdot i}\right\|_{2} & \leq \widetilde{c h}\left\|E_{. i}\right\|_{2}+|\widetilde{s h}|\left\|E_{\cdot j}\right\|_{2} \\
& \leq\left(\left|\varepsilon_{1}^{\prime}\right| \widetilde{c h}^{2}+\left|\varepsilon_{3}^{\prime}\right| \widetilde{s h}^{2}\right)\left\|G_{\cdot i}\right\|_{2}+\left(\left|\varepsilon_{2}^{\prime}\right|+\left|\varepsilon_{4}^{\prime}\right|\right) \widetilde{c h}|\widetilde{s h}|\left\|G_{\cdot j}\right\|_{2} \\
& \leq\left(\left|\varepsilon_{1}^{\prime}\right| \widetilde{c h}^{2}+\left|\varepsilon_{3}^{\prime}\right| \widetilde{s h}^{2}+\left(\left|\varepsilon_{2}^{\prime}\right|+\left|\varepsilon_{4}^{\prime}\right|\right) \widetilde{c h}|\widetilde{s h}| x\right) d_{i}
\end{aligned}
$$

and

$$
\begin{aligned}
\left\|F_{\cdot j}\right\|_{2} & \leq|\widetilde{s h}| \cdot\left\|E_{\cdot i}\right\|_{2}+\widetilde{c h}\left\|E_{\cdot j}\right\|_{2} \\
& \leq\left(\left|\varepsilon_{4}^{\prime}\right| \widetilde{c h}^{2}+\left|\varepsilon_{2}^{\prime}\right| \widetilde{s h}^{2}\right)\left\|G_{\cdot j}\right\|_{2}+\left(\left|\varepsilon_{1}^{\prime}\right|+\left|\varepsilon_{3}^{\prime}\right|\right) \widetilde{c h}|\widetilde{s h}|\left\|G_{\cdot i}\right\|_{2} \\
& \leq\left(\left|\varepsilon_{4}^{\prime}\right| \widetilde{c h}^{2}+\left|\varepsilon_{2}^{\prime}\right| \widetilde{s h}^{2}+\left(\left|\varepsilon_{1}^{\prime}\right|+\left|\varepsilon_{3}^{\prime}\right|\right) \widetilde{c h}|\widetilde{s h}| \frac{1}{x}\right) d_{j} .
\end{aligned}
$$

Now we have to calculate the upper bounds for $\left|\varepsilon_{i}^{\prime}\right|$ 's, $\widetilde{c h}^{2}, \widetilde{s h}^{2}$ and $\widetilde{c h}|\widetilde{s h}|$, and to insert them into relations (3.3.16) and (3.3.17). We consider two cases, $|\zeta| \leq \alpha$ and $|\zeta|>\alpha$, where $\alpha$ is defined by (3.2.17).

First consider $|\zeta| \leq \alpha$. As in the proof of Th. 3.2.1 we can show that the relations (3.2.18) and (3.2.20) hold. From (3.2.11) it follows that

$$
f l\left(\sqrt{1-t^{2}}\right)=\left(1+\varepsilon_{h}\right) \sqrt{1-t^{2}},
$$

where

$$
\left|\varepsilon_{h}\right| \leq\left(\frac{3}{8} \sqrt{\kappa}+\frac{4}{3}\right) \varepsilon
$$

Therefore,

$$
\left|\varepsilon_{s h}\right|,\left|\varepsilon_{c h}\right| \leq\left(\frac{3}{8} \sqrt{\kappa}+\frac{7}{3}\right) \varepsilon
$$

so that

$$
\left|\varepsilon_{i}^{\prime}\right| \leq\left(\frac{3}{8} \sqrt{\kappa}+\frac{13}{3}\right) \varepsilon, \quad i=1, \ldots, 4 .
$$

Inserting $1 / x \leq \sqrt{2},(3.2 .20)$, and the above relation in (3.3.16) and (3.3.17), we obtain

$$
\begin{aligned}
& \left\|F_{\cdot i}\right\|_{2} \leq(0.375 \kappa+5.46 \sqrt{\kappa}+13) d_{i} \varepsilon \\
& \left\|F_{\cdot j}\right\|_{2} \leq(0.46 \kappa+6.6 \sqrt{\kappa}+15.67) d_{j} \varepsilon .
\end{aligned}
$$

This, in turn, implies $\left\|\delta B_{m}\right\|_{2} \leq C_{m}$ as desired.

Now consider the case $|\zeta|>\alpha$. As in the proof of Th. 3.2.1, we can show that the relations (3.2.23) hold for $t, \widetilde{c h}$ and $\widetilde{s h}$ with a relative error of $O(\varepsilon)$. Now

$$
f l\left(\sqrt{1-t^{2}}\right)=\left(1+\varepsilon_{h}\right) \sqrt{1-t^{2}}, \quad\left|\varepsilon_{h}\right| \leq 3 \varepsilon,
$$

so that

$$
\left|\varepsilon_{s h}\right|,\left|\varepsilon_{c h}\right| \leq 4 \varepsilon, \quad\left|\varepsilon_{i}^{\prime}\right| \leq 6 \varepsilon, \quad i=1, \ldots, 4
$$


We have two subcases, $x \geq \beta$ and $x<\beta$, where $\beta$ is defined by (3.2.17). If $x \geq \beta$, then inserting $1 / x \leq \sqrt{2},(3.3 .18)$ and (3.2.23) into (3.3.16) and (3.3.17) yields

$$
\left\|F_{. i}\right\|_{2} \leq 35 d_{i} \varepsilon, \quad\left\|F_{. j}\right\|_{2} \leq 42 d_{j} \varepsilon, \quad\left\|\delta B_{m}\right\|_{2} \leq 77 \varepsilon
$$

as desired.

If $x<\beta$, then

$$
\begin{aligned}
|t| & \leq \frac{1}{|\zeta|}=\frac{2|c|}{|a+b|}=\frac{2\left|c_{T}+\varepsilon_{c} \sqrt{a_{T} b_{T}}\right|}{\left|a_{T}+\varepsilon_{a} a_{T}+b_{T}+\varepsilon_{b} b_{T}\right|} \\
& =\frac{2\left|z x+\varepsilon_{c} x\right|}{\left|1+x^{2}+\varepsilon_{a}+\varepsilon_{b} x^{2}\right|} \leq 2 x(z+n \varepsilon)(1+O(\varepsilon)) .
\end{aligned}
$$

We can ignore the $(z+n \varepsilon)(1+O(\varepsilon))$ term, so that $|t| \leq 2 x$. Therefore,

$$
\widetilde{c h}|\widetilde{s h}|=\widetilde{c h}^{2}|t| \leq 2 \widetilde{c h}^{2} x
$$

Inserting this, (3.3.18) and (3.2.23) into (3.3.16) and (3.3.17), we obtain

$$
\left\|F_{. i}\right\|_{2} \leq 30 d_{i} \varepsilon, \quad\left\|F_{. j}\right\|_{2} \leq 66 d_{j} \varepsilon, \quad\left\|\delta B_{m}\right\|_{2} \leq 96 \varepsilon
$$

and the theorem is proved.

Q.E.D.

Corollary 3.3.4 Assume Algorithm 3.3.1 converges, and that $G_{M}, J$ is the final pair which satisfies the stopping criterion. For $0 \leq m \leq M$ write $G_{m}=B_{m} D_{m}$ with $D_{m}$ diagonal and $B_{m}$ with unit columns. Let $\lambda_{j}$ be the $j$-th non-zero eigenvalue of $G_{0} J G_{0}^{T}$, and let $\lambda_{j}^{\prime}$ be the $j$-th computed non-zero eigenvalue. Then, with the relative error of $O(\varepsilon)$,

$$
(1-\gamma)^{2} \leq \frac{\lambda_{j}^{\prime}}{\lambda_{j}} \leq(1+\gamma)^{2}
$$

where

$$
\gamma=\varepsilon \sum_{m=0}^{M-1} \frac{C_{m}}{\sigma_{\min }\left(B_{m}\right)}+n \cdot \mathrm{tol} / 2+r \cdot n \cdot \varepsilon / 2 .
$$

Proof. Let $\lambda_{m, j}$ denote the $j$-th non-zero eigenvalue of the matrix $G_{m} J G_{m}^{T}$. By substituting (3.3.3) into (2.3.12) and then applying Th. 2.3.1 for every $0 \leq m \leq M-1$, we obtain

$$
\left(1-\eta_{m}\right)^{2} \leq \frac{\lambda_{m+1, j}}{\lambda_{m, j}} \leq\left(1+\eta_{m}\right)^{2}
$$

where

$$
\eta_{m}=\varepsilon C_{m} / \sigma_{\min }\left(B_{m}\right)
$$

Also,

$$
\operatorname{diag}\left(\lambda_{j}^{\prime}\right)=\operatorname{diag}\left(f l\left(G_{M}^{T} G_{M}\right)\right)=f l\left(G_{M}^{T} G_{M}\right)+F
$$


where

$$
\left|F_{i j}\right| \leq(n \varepsilon+t o l)\left\|G_{M \cdot i}\right\|_{2}\left\|G_{M, \cdot j}\right\|_{2} .
$$

Here $G_{M, i}$ denotes the $i$-th column of $G_{M}$. The tol term comes from the stopping criterion. The $n \varepsilon$ term comes for the off-diagonal elements of $F$ from the fact that $c / \sqrt{a b}$ in the stopping criterion may be underestimated by as much as $n \varepsilon$, and for the diagonal elements of $F$ from computing the norms of the columns of $G_{M}$. Therefore,

$$
1-r \cdot n \cdot \varepsilon-n \cdot t o l \leq \frac{\lambda_{j}^{\prime}}{\lambda_{M, j}} \leq 1+r \cdot n \cdot \varepsilon+n \cdot t o l
$$

and (3.3.20) follows by inserting (3.3.21) and the above relation into (3.2.31), and ignoring the relative error of $O(\varepsilon)$.

Q.E.D.

An alternative way to prove this corollary is given in the proof of Th. 3.3.9.

Remark 3.3.5 If $G$ is square and non-singular, then Cor. 3.3.4 can be applied to the hyperbolic singular value problem. Let $\sigma_{j}$ be the $j$-th hyperbolic singular value of $G_{0}, J$ and $\sigma_{j}^{\prime}$ the $j$-th computed hyperbolic singular value. Then, by taking square roots in (3.3.20) and ignoring relative errors of $O(\varepsilon)$, we obtain

$$
1-\gamma-\varepsilon \leq \frac{\sigma_{j}^{\prime}}{\sigma_{j}} \leq 1+\gamma+\varepsilon
$$

where

$$
\gamma=\varepsilon \sum_{m=0}^{M-1} \frac{C_{m}}{\sigma_{\min }\left(B_{m}\right)}+n \cdot t o l / 2+r \cdot n \varepsilon / 2
$$

Extra $\varepsilon$ in (3.3.22) comes from the fact that $\sigma_{j}^{\prime}=f l\left(\sqrt{\lambda_{j}^{\prime}}\right)$.

This remark holds for all subsequent implicit methods in this chapter.

$æ$ 
As we did in Subsect. 3.2.1, we can modify the implicit $J$-orthogonal Jacobi method in order to avoid potentially large $C_{m}$ in Th. 3.4.2 in the hyperbolic case for $|\zeta| \leq \alpha$. The algorithm of the modified method is obtained by combining Algorithms 3.3.1 and 3.2.7 in the obvious manner. The comments from Subsect. 3.2.1 hold here, as well. We have the following:

Theorem 3.3.6 Let $G_{m}$ be the sequence of matrices generated by the modified implicit J-orthogonal Jacobi method in finite precision arithmetic with precision $\varepsilon$. Then Theorem 3.3.3 holds except that in the hyperbolic case for $|\zeta| \leq 3 /(2 \sqrt{2})$ the value $C_{m}$ is changed to $C_{m}=28$. Corollary 3.3.4 holds with this exception, too.

Proof. The technique of proof is the same as in Th. 3.3.3. We assume without loss of generality that $\widetilde{s h}=s h=+1$. Also, $\widetilde{c h}=\sqrt{2}, \operatorname{ch}=f l(\sqrt{2})$, so that

$$
\left|\varepsilon_{c h}\right| \leq \varepsilon, \quad \varepsilon_{s h}=0 .
$$

Therefore,

$$
\left|\varepsilon_{1}^{\prime}\right|,\left|\varepsilon_{4}^{\prime}\right| \leq 3 \varepsilon, \quad\left|\varepsilon_{2}^{\prime}\right|,\left|\varepsilon_{3}^{\prime}\right| \leq 2 \varepsilon,
$$

and the theorem follows by inserting these values and $1 / x \leq \sqrt{2}$ into (3.3.16) and (3.3.17).

Q.E.D.

\subsubsection{Keeping the diagonal in a separate vector}

The approximate operation count for the implicit $J$-orthogonal Jacobi method of Alg. 3.3.1 is the following: we need $3 n$ multiplications and $3(n-1)$ additions to calculate $a, b$, and $c$, and $4 n$ multiplications and $2 n$ additions to update vectors $G_{. i}, G_{. j}$ per rotation. This gives the total of approximately $3.5 n^{3}$ multiplications and $2.5 n^{3}$ additions per cycle $(n(n-1) / 2$ rotations). Keeping the diagonal elements of the matrix $G^{T} G$ in a separate vector makes the calculation of the parameters $a$ and $b$ via scalar product in each step unnecessary, which leaves the total of $2.5 n^{3}$ multiplications and $1.5 n^{3}$ additions per cycle.

The main idea (in the notation of Alg. 3.3.1) is the following: at the beginning of each cycle we calculate

$$
\Delta_{i}=\sum_{k=1}^{n} G_{k i}^{2} .
$$

At the beginning of each step we set

$$
a=\Delta_{i}, \quad b=\Delta_{j}, \quad c=\sum_{k=1}^{n} G_{k i} G_{k j} .
$$

We update $\Delta_{i}$ and $\Delta_{j}$ by the formulae

$$
\Delta_{i}=\Delta_{i}-c * t, \quad \Delta_{j}=\Delta_{j}+c * t,
$$


in the trigonometric, and

$$
\Delta_{i}=\Delta_{i}+c * t, \quad \Delta_{j}=\Delta_{j}+c * t
$$

in the hyperbolic case, respectively.

Due to subtractions in updating $\Delta_{i}$ 's, they can become inaccurate, i.e. the relative error of $\Delta_{i}$ to $\left\|G_{. i}\right\|^{2}$ can be larger then $O(\varepsilon)$. Suppose that $\Delta_{i}=\left\|G_{. i}\right\|^{2}$. After one subtraction we have

$$
\Delta_{i}^{\prime}=\left\|G_{. i}^{\prime}\right\|^{2}(1+\epsilon), \quad|\epsilon| \leq \frac{\kappa^{2}\left(B_{m}\right)+1}{2} \varepsilon,
$$

where the maximum is attained when $z$ tends to its upper bound (3.3.4) and $a=$ $b$. Therefore, the relative error of $\Delta_{i}$ can grow considerably, which can affect the convergence by making the rotation angles inaccurate. This is why the vector $\Delta$ should be updated at the beginning of each cycle from the columns of the current matrix $G$. We did not use the well known Rutishauser's delayed updates of the diagonal, since they do not guarantee high relative accuracy of the diagonal at the beginning of each cycle.

When the pair $G^{T} G, J$ is obtained from the pair $G J G^{T}, I$, then the probability that the convergence is actually spoiled is very low. This is due to a non-trivial diagonalizing effect of the above transition.

We now turn to the one-step error analysis of the method. In the notation of Th. 3.3.3 we have

$$
\Delta_{i}=d_{i}^{2}, \quad \Delta_{j}=d_{j}^{2}, \quad x=\sqrt{\Delta_{i} / \Delta_{j}} .
$$

If $x \geq \bar{x}$ in the trigonometric, and $x \geq \beta$ in the hyperbolic case, then Th. 3.3.3 holds irrespectively of the accuracy of $\Delta_{i}$ and $\Delta_{j}$.

If $x<\bar{x}$ in the trigonometric, and $x<\beta$ in the hyperbolic case, then Th. 3.3.3 holds if the relations (3.3.10) and (3.3.19) are satisfied, respectively. This is always the case if

$$
c^{2}<\Delta_{i} \Delta_{j}
$$

If the above inequality does not hold, then we have to refresh $\Delta_{i}$ and $\Delta_{j}$. Note that hyperbolic rotations cause no additional problems over trigonometric ones.

The following algorithm is only a slight modification of Alg. 3.3.1, so only the parts where the two algorithms differ are stated.

Algorithm 3.3.7 Implicit $J$-orthogonal method for the pair $G, J$. The vector $\Delta$ contains diagonal elements of the matrix $G^{T} G$.

repeat

${ }^{*}$ at the beginning of each cycle refresh the vector

$\Delta$ which contains diagonal of $G^{T} G * /$

for $j=1$ to $r$ 
endfor

$$
\Delta_{j}=\sum_{k=1}^{n} G_{k j}^{2}
$$

for all pairs $i<j$

$$
\begin{aligned}
& { }^{*} \text { compute }\left[\begin{array}{ll}
a & c \\
c & b
\end{array}\right] \equiv \text { the }(i, j) \text { submatrix of } G^{T} G * / \\
& c=\sum_{k=1}^{n} G_{k i} * G_{k j} \\
& \text { if } c^{2}<\Delta_{i} \Delta_{j} \text { then } \\
& a=\Delta_{i} \\
& b=\Delta_{j} \\
& a=\sum_{k=1}^{n} G_{k i}^{2} \\
& \text { endif } \\
& b=\sum_{k=1}^{n} G_{k j}^{2}
\end{aligned}
$$

${ }^{*}$ compute the parameter hyp: hyp $=1$ for the hyperbolic and hyp $=-1$ for the trigonometric rotation, respectively */

${ }^{*}$ compute the $J$-orthogonal Jacobi rotation which diagonalizes

$$
\left[\begin{array}{ll}
H_{i i} & H_{i j} \\
H_{j i} & H_{j j}
\end{array}\right] \equiv\left[\begin{array}{cc}
a & c \\
c & b
\end{array}\right] * /
$$

$/^{*}$ update columns $i$ and $j$ of $G * /$

${ }^{*}$ update $\Delta_{i}$ and $\Delta_{j} * /$

$$
\begin{aligned}
& \Delta_{i}=a+h y p * c * t \\
& \Delta_{j}=b+c * t
\end{aligned}
$$

endfor

until convergence $($ all $|c| / \sqrt{a b} \leq$ tol $)$

${ }^{*}$ the computed non-zero eigenvalues of $H=G J G^{T}$ (and of the pair $G^{T} G, J$ ) are

$$
\lambda_{j}=\left(\sum_{k=1}^{n} G_{k j}^{2}\right) J_{j j} * /
$$

$I^{*}$ the computed eigenvectors of $H$ are the normalized columns of the final $G * /$

Numerical experiments of Chap. 5 showed no difference in the accuracy between Alg. 3.3.7 and other implicit algorithms.

$æ$ 


\subsubsection{Error bounds for the eigenvectors}

Theorems which give one-step error analysis of the implicit $J$-orthogonal Jacobi methods in Sections 3.3 and 3.4 imply that one step of any of those methods satisfies the eigenprojection perturbation bounds of Th. 2.3.3. As a consequence, the eigen (spectral) projections computed by any of those methods also satisfy those bounds. We prove the following theorem for the method defined by Alg. 3.3.1. The proof for other implicit methods is similar. In the proof of the theorem we use the following lemma due to Veselić [30]:

Lemma 3.3.8 Let

$$
F^{*} F=I+E, \quad\|E\|_{2}=\epsilon<1,
$$

where $F$ is any matrix with full column rank. Then there exists a matrix $Q$ such that $Q^{*} Q=I$ and $\|F-Q\|_{2} \leq \epsilon$.

Proof. We make the polar decomposition $F=Q P$ where $Q^{*} Q=I$ and $P$ is Hermitian positive definite matrix. Since $Q Q^{*} F=F$, we have $P^{2}=I+E$, or

$$
(P+I)(P-I)=E \text {. }
$$

Thus

$$
\|P-I\|_{2} \leq \epsilon /(1+\sqrt{1-\epsilon}) \leq \epsilon
$$

so that

$$
\|F-Q\|_{2}=\|Q P-Q\|_{2}=\|P-I\|_{2},
$$

and the lemma is proved.

Q.E.D.

Theorem 3.3.9 Let $G, J$, where $G$ is non-singular, be the starting pair for Alg. 3.3.1. Assume algorithm converges, and that $G_{M}, J$ is the final pair which satisfies the stopping criterion. For $0 \leq m \leq M$ write $G_{m}=B_{m} D_{m}$, where $D_{m}$ diagonal and $B_{m}$ has unit columns. Let $\lambda$ be an eigenvalue of the matrix $G J G^{T}$ and let $P$ be its eigenprojection. Let $P^{\prime}$ be the approximation of the corresponding spectral projection, i.e. $P^{\prime}$ is obtained from the final eigenvectors which are obtained by dividing the columns of $G_{M}$ by their norms. Then, with the relative error of $O(\varepsilon)$,

$$
\left\|P^{\prime}-P\right\|_{2} \leq \frac{4 \bar{\eta}}{r g_{G}(\lambda)} \frac{1}{1-\frac{3 \bar{\eta}}{r g_{G}(\lambda)}}+2 n \cdot \operatorname{tol}+n(3 n+4) \varepsilon,
$$

where $\bar{\eta}=\eta(\eta+2)$, and

$$
\eta=\varepsilon \sum_{m=0}^{M-1} \frac{C_{m}}{\sigma_{\min }\left(B_{m}\right)}+n \cdot t o l+n^{2} \varepsilon,
$$

provided $3 \bar{\eta} / r g_{G}(\lambda)<1$. Here $r g_{G}(\lambda)$ is defined by (2.3.1) and the quantities $C_{m}$ are defined by Th. 3.3.3. 
Proof. We first show that for every $1 \leq m \leq M$, the matrix $G_{m}$ is obtained by the sequence of exact transformations on some perturbed matrix $G+\delta G^{(m-1)}$ in the sense of Th. 3.3.3, i.e.

$$
G_{m}=\left(G+\delta G^{(m-1)}\right) R_{0} \cdots R_{m-1}
$$

where

$$
\left\|\delta G^{(m-1)} x\right\|_{2} \leq \varepsilon \sum_{k=0}^{m-1} \frac{C_{k}}{\sigma_{\min }\left(B_{k}\right)}\|G x\|_{2}
$$

holds with the relative error of $\varepsilon$. The proof is by induction on $m$. For $m=1$ the statement follows from Th. 3.3.3. Now suppose that (3.3.24) holds for some $m \geq 1$. By Th. 3.3.3 and the induction assumption we have

$$
\begin{aligned}
G_{m+1} & =\left(G_{m}+\delta G_{m}\right) R_{m} \\
& =\left[\left(G+\delta G^{(m-1)}\right) R_{0} \cdots R_{m-1}+\delta G_{m}\right] R_{m} \\
& =\left(G+\delta G^{(m)}\right) R_{0} \cdots R_{m}
\end{aligned}
$$

where

$$
\delta G^{(m)}=\delta G^{(m-1)}+\delta G_{m}\left(R_{0} \cdots R_{m-1}\right)^{-1} .
$$

Set $\delta G_{m}=\delta B_{m} D_{m}$. Then

$$
\left\|\delta G^{(m)} x\right\|_{2} \leq\left\|\delta G^{(m-1)} x\right\|_{2}+\frac{\left\|\delta B_{m}\right\|_{2}}{\sigma_{\min }\left(B_{m}\right)}\left\|B_{m} D_{m} G_{m}^{-1}\left(G+\delta G^{(m-1)}\right) x\right\|_{2},
$$

and (3.3.24) follows from (3.3.25) and Th. 3.3.3, ignoring the relative errors of $O(\varepsilon)$.

Since the final pair satisfies the stopping criterion, we have

$$
B_{M}^{T} B_{M}=I+E, \quad\|E\|_{2} \leq n \cdot t o l+n^{2} \varepsilon .
$$

The $n^{2} \varepsilon$ term comes from the fact that $c / \sqrt{a b}$ in the stopping criterion may be underestimated by as much as $n \varepsilon$. Lemma 3.3.8 implies that there exists an orthogonal matrix

$$
B_{M}^{\prime}=B_{M}+\delta B_{M}
$$

where

$$
\left\|\delta B_{M}\right\|_{2} \leq n \cdot t o l+n^{2} \varepsilon .
$$

Set $G_{M}^{\prime}=B_{M}^{\prime} D_{M}$. As in the first part of the proof, we can show that

$$
G_{M}^{\prime}=\left(G+\delta G^{(M)}\right) R_{0} \cdot \ldots \cdot R_{M-1}
$$

where $\left\|\delta G^{(M)} x\right\|_{2} \leq \eta\|G x\|_{2}$. Since $\sigma_{\min }\left(B_{M}\right) \geq 1-\left(n \cdot t o l+n^{2} \varepsilon\right)$, we ignore the factor $1 / \sigma_{\min }\left(B_{M}\right)$ when applying Th. 3.3.3. Let $P_{M}^{\prime}$ denote the spectral projection 
of the matrix $G_{M}^{\prime} J G_{M}^{\prime T}$ which corresponds to the eigenprojection $P$. Th. 2.3.3 now implies

$$
\left\|P-P_{M}^{\prime}\right\|_{2} \leq \frac{4 \bar{\eta}}{r g_{G}(\lambda)} \cdot \frac{1}{1-3 \bar{\eta} / r g_{G}(\lambda)}
$$

The spectral projection $P_{M}^{\prime}$ is obtained from columns of the matrix $B_{M}^{\prime}$, while the approximation $P^{\prime}$ is obtained from columns of the matrix

$$
f l\left(G_{M} \cdot\left|\operatorname{diag}\left(\lambda_{j}^{\prime}\right)\right|^{-1 / 2}\right)=B_{M}+F
$$

where

$$
\left|F_{i j}\right| \leq\left|B_{M, i j}\right|(n+4) \varepsilon / 2 .
$$

Here we used $\left|\lambda_{j}^{\prime}\right| / D_{M, j} \leq 1+n \varepsilon$ and ignored the relative error of $O(\varepsilon)$. Using $\left\|B_{M}\right\|_{2} \leq 1+n \cdot t o l+n^{2} \varepsilon$, and ignoring again the relative error of $O(\varepsilon)$, we finally have

$$
\begin{aligned}
\left\|P_{M}^{\prime}-P^{\prime}\right\|_{2} & \leq\left\|\left(B_{M}+\delta B_{M}\right)\left(B_{M}+\delta B_{M}\right)^{T}-\left(B_{M}+F\right)\left(B_{M}+F\right)^{T}\right\|_{2} \\
& \leq 2\left\|\delta B_{M}\right\|_{2}+2\|F\|_{2} \\
& \leq 2 n \cdot \operatorname{tol}+n(3 n+4) \varepsilon
\end{aligned}
$$

which, together with (3.3.26), implies (3.3.23).

Q.E.D.

$æ$ 


\subsection{Fast implicit method}

In this section we define and analyse the fast implicit $J$-orthogonal Jacobi method for the pair $G, J$. The remarks from Sect. 3.3 hold here as well. The section is also organized as Sect. 3.3. We first present the algorithm. We then give one-step error analysis and overall error bound for the eigenvalues. In Th. 3.4.4 we give one-step error analysis of the modified method. After that we shortly discuss the version of the algorithm where the diagonal of $G^{T} G$ is kept in a separate vector. In Subsect. 3.4.1 we consider fast self-scaling rotations used in order to avoid possible underflow/overflow when updating the scaling matrix.

The idea of fast rotations is to use transformation matrices of the form

$$
J_{m}=\left[\begin{array}{ll}
1 & \alpha \\
\beta & 1
\end{array}\right]
$$

instead of matrices of the form

$$
\left[\begin{array}{cc}
c s & s n \\
-s n & c s
\end{array}\right], \quad\left[\begin{array}{cc}
c h & s h \\
s h & c h
\end{array}\right] .
$$

This saves $2 n$ multiplications in each step, or approximately $n^{3}$ multiplications in each cycle. The use of matrices of the type (3.4.1) is possible if the matrices $G_{m}$ are stored in factorized form

$$
G_{m}=\bar{G}_{m} \bar{D}_{m}
$$

where $\bar{D}_{m}$ is diagonal positive definite.

In the $m$-th step of the implicit method only the columns $i$ and $j$ of the matrix $G_{m}$ are changed. Let $G_{m} \equiv G$ and $G_{m+1} \equiv G^{\prime}$. If we use the ordinary rotation, then we have

$$
\left[\begin{array}{ll}
G_{\cdot i}^{\prime} & G_{\cdot j}^{\prime}
\end{array}\right]=\left[\begin{array}{ll}
G_{\cdot i} & G_{\cdot j}
\end{array}\right]\left[\begin{array}{cc}
c s & s n \\
-s n & c s
\end{array}\right],
$$

in the trigonometric, or

$$
\left[\begin{array}{ll}
G_{\cdot i}^{\prime} & G_{\cdot j}^{\prime}
\end{array}\right]=\left[\begin{array}{ll}
G_{\cdot i} & G_{\cdot j}
\end{array}\right]\left[\begin{array}{cc}
c h & s h \\
s h & c h
\end{array}\right]
$$

in the hyperbolic case. Now suppose that $G=\bar{G} \bar{D}$, i.e.

$$
\left[\begin{array}{ll}
G_{\cdot i} & G_{\cdot j}
\end{array}\right]=\left[\begin{array}{ll}
\bar{G}_{\cdot i} & \bar{G}_{\cdot j}
\end{array}\right]\left[\begin{array}{ll}
\bar{D}_{i} & \\
& \bar{D}_{j}
\end{array}\right] .
$$

Simple calculation shows that

$$
\left[\begin{array}{ll}
G_{\cdot i}^{\prime} & G_{\cdot j}^{\prime}
\end{array}\right]=\left[\begin{array}{ll}
\bar{G}_{\cdot i}^{\prime} & \bar{G}_{\cdot j}^{\prime}
\end{array}\right]\left[\begin{array}{cc}
\bar{D}_{i}^{\prime} & \\
& \bar{D}_{j}^{\prime}
\end{array}\right],
$$


where

$$
\left[\begin{array}{ll}
\bar{G}_{\cdot i}^{\prime} & \bar{G}_{\cdot j}^{\prime}
\end{array}\right]=\left[\begin{array}{ll}
\bar{G}_{\cdot i} & \bar{G}_{\cdot j}
\end{array}\right]\left[\begin{array}{cc}
1 & \alpha \\
\beta & 1
\end{array}\right]
$$

Here

$$
\begin{array}{lc}
\alpha=\frac{\bar{D}_{i}}{\bar{D}_{j}} t, & \beta=-\frac{\bar{D}_{j}}{\bar{D}_{i}} t, \quad t=s n / c s, \\
\bar{D}_{i}^{\prime}=\bar{D}_{i} c s, & \bar{D}_{j}^{\prime}=\bar{D}_{j} c s,
\end{array}
$$

in the trigonometric, and

$$
\begin{aligned}
& \alpha=\frac{\bar{D}_{i}}{\bar{D}_{j}} t, \quad \beta=\frac{\bar{D}_{j}}{\bar{D}_{i}} t, \quad t=s h / c h, \\
& \bar{D}_{i}^{\prime}=\bar{D}_{i} c h, \quad \bar{D}_{j}^{\prime}=\bar{D}_{j} c h,
\end{aligned}
$$

in the hyperbolic case.

We now state the algorithm: 
Algorithm 3.4.1 Fast implicit J-orthogonal Jacobi method for the pair G, J. tol is a user defined stopping criterion.

for $k=1$ to $r$

$$
D_{k}=1
$$

endfor

repeat

$$
\begin{aligned}
& \text { for all pairs } i<j
\end{aligned}
$$

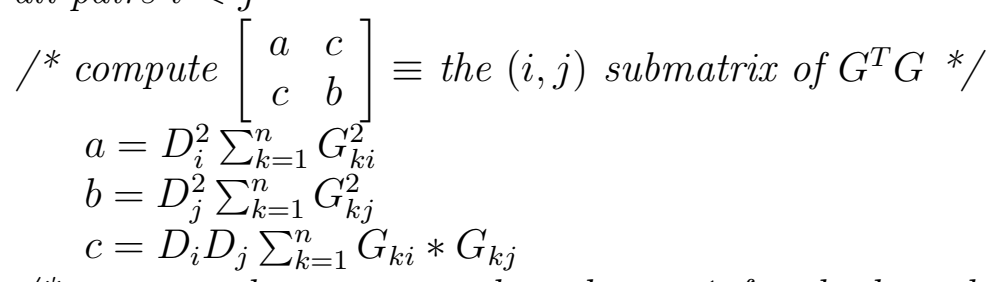

${ }^{*}$ compute the parameter hyp: hyp $=1$ for the hyperbolic and hyp $=-1$ for the trigonometric rotation, respectively */

if $1 \leq i \leq$ npos $<j \leq r$ then

$$
\text { hyp }=1
$$

else

$$
\text { hyp }=-1
$$

endif

${ }^{*}$ compute the J-orthogonal Jacobi rotation which diagonalizes

$$
\begin{aligned}
& {\left[\begin{array}{ll}
H_{i i} & H_{i j} \\
H_{j i} & H_{j j}
\end{array}\right] \equiv\left[\begin{array}{ll}
a & c \\
c & b
\end{array}\right] * /} \\
& \zeta=-h y p *(b+h y p * a) /(2 c) \\
& t=\operatorname{sign}(\zeta) /\left(|\zeta|+\sqrt{\zeta^{2}-h y p}\right) \\
& c s=1 / \sqrt{1-h y p * t^{2}} \\
& \alpha=t * D_{i} / D_{j} \\
& \beta=h y p * t * D_{j} / D_{i} \\
& /^{*} \text { update columns } i \text { and } j \text { of } G * / \\
& \text { for } k=1 \text { to } n \\
& \quad \text { tmp }=G_{k i} \\
& \quad G_{k i}=\text { tmp }+\alpha * G_{k j} \\
& G_{k j}=\beta * \operatorname{tmp}+G_{k j} \\
& \text { endfor } \\
& /^{*} \text { update } D_{i} \text { and } D_{j} * / \\
& D_{i}=D_{i} * c s \\
& D_{j}=D_{j} * c s
\end{aligned}
$$

endfor

until convergence $($ all $|c| / \sqrt{a b} \leq$ tol $)$

${ }^{*}$ the computed non-zero eigenvalues of $H=G J G^{T}$ (and of the pair $G^{T} G, J$ ) are

$$
\lambda_{j}=\left(\sum_{k=1}^{n} G_{k j}^{2}\right) D_{j}^{2} J_{j j} * /
$$

${ }^{*}$ the computed eigenvectors of $H$ are the normalized columns of the final $G * /$ 
The following theorem and its corollary justify our accuracy claims for the eigenvalues of the matrix $H=G J G^{T}$ computed by the fast implicit $J$-orthogonal Jacobi method .

Theorem 3.4.2 Let $\bar{G}_{m}, \bar{D}_{m}$ be the sequences of matrices generated by the fast implicit J-orthogonal Jacobi algorithm in floating-point arithmetic with precision $\varepsilon$; that is $\bar{G}_{m+1}$ is obtained from $\bar{G}_{m}$ by applying a single fast rotation, and $D_{m+1}$ is obtained from $D_{m}$ according to (3.4.3) or (3.4.4). Let $G_{m} \equiv \bar{G}_{m} \cdot \bar{D}_{m}$. Since $G_{m}$ is needed only for theoretical consideration, we suppose that this matrix multiplication is exact. Then the following diagram commutes.

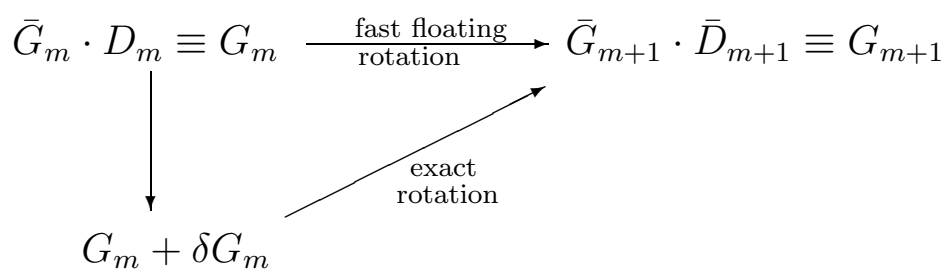

The top arrow indicates that $G_{m+1}$ is obtained from $G_{m}$ by applying one fast rotation in floating-point arithmetic. The diagonal arrow indicates that $G_{m+1}$ is obtained from $G_{m}+\delta G_{m}$ by applying one $J$-orthogonal plane rotation in exact arithmetic; thus $G_{m+1} J G_{m+1}$ and $\left(G_{m}+\delta G_{m}\right) J\left(G_{m}+\delta G_{m}\right)^{T}$ have identical eigenvalues. $\delta G_{m}$ is bounded as follows. Let $\kappa=\kappa^{2}\left(B_{m}\right)$ and write $\delta G_{m}=\delta B_{m} D_{m}$, where $D_{m}$ is diagonal such that $B_{m}$ in $G_{m}=B_{m} D_{m}$ has unit columns. Let $a_{T}$ and $b_{T}$ be the true values of $\sum_{k} G_{k i}^{2}$ and $\sum_{k} G_{k j}^{2}$, respectively. Then, with the relative error of order $\varepsilon$,

$$
\left\|\delta B_{m}\right\|_{2} \leq C_{m} \cdot \varepsilon
$$

where

$$
C_{m}=\left\{\begin{array}{lll}
33 & \text { in trigonometric case , } & \\
\kappa+16 \sqrt{\kappa}+39 & \text { in hyperbolic case, } & |\zeta| \leq \frac{3}{2 \sqrt{2}}, \\
102 & \text { in hyperbolic case, } & |\zeta|>\frac{3}{2 \sqrt{2}}, \\
125 & \text { in hyperbolic case, } & |\zeta|>\frac{1}{2} a_{T}, \\
& & b_{T}<\frac{1}{2} a_{T} .
\end{array}\right.
$$

In other words, one step of the fast implicit J-orthogonal Jacobi method satisfies the assumptions needed for the perturbation bounds of Sect. 2.3.

Proof. The proof of the commuting diagram is a tedious computation. We shall prove the diagram separately for the trigonometric and for the hyperbolic case. Let 
$a_{T}, b_{T}$ and $c_{T}$ be the true values of $\bar{D}_{i}^{2} \sum_{k} \bar{G}_{k i}^{2}, \bar{D}_{j}^{2} \sum_{k} \bar{G}_{k j}^{2}$ and $\bar{D}_{i} \bar{D}_{j} \sum_{k} \bar{G}_{k i} \bar{G}_{k j}$. We may assume without loss of generality that $a_{T} \geq b_{T}$ and $c_{T}>0$. As in (3.2.3), we have

$$
a_{T}=d_{i}^{2}, \quad b_{T}=d_{j}^{2}, \quad c_{T}=z d_{i} d_{j} .
$$

As in (3.2.4), we can show that (3.3.4) holds. Also let $x \equiv d_{j} / d_{i} \leq 1$. Systematic application of formulae (3.2.1) shows that

$$
\begin{array}{rrr}
a=a_{T}\left(1+\varepsilon_{a}\right) & \text { where } & \left|\varepsilon_{a}\right| \leq(n+2) \varepsilon \\
b=b_{T}\left(1+\varepsilon_{b}\right) & \text { where } & \left|\varepsilon_{b}\right| \leq(n+2) \varepsilon \\
c=c_{T}+\varepsilon_{c} \sqrt{a_{T} b_{T}} & \text { where } & \left|\varepsilon_{c}\right| \leq(n+2) \varepsilon
\end{array}
$$

Trigonometric case. This case was analysed by Anda and Park [1] for the Givens rotation in the QR-algorithm. Our proof is similar to theirs.

Let

$$
\begin{aligned}
\widetilde{c s} & \equiv 1 / \sqrt{1+t^{2}}, & \widetilde{s n} \equiv t / \sqrt{1+t^{2}} \\
\widetilde{\alpha} & \equiv t \bar{D}_{i} / \bar{D}_{j}, & \widetilde{\beta} \equiv-t \bar{D}_{j} / \bar{D}_{i} .
\end{aligned}
$$

For the calculated transformation parameters we have

$$
\begin{array}{rlrl}
c s & =\left(1+\varepsilon_{c s}\right) \widetilde{c s}, & & \left|\varepsilon_{c s}\right| \leq 3 \varepsilon, \\
\alpha=\left(1+\varepsilon_{\alpha}\right) \widetilde{\alpha}, & \beta=\left(1+\varepsilon_{\beta}\right) \widetilde{\beta}, & \left|\varepsilon_{\alpha}\right|,\left|\varepsilon_{\beta}\right| \leq 2 \varepsilon .
\end{array}
$$

$\widetilde{c s}$ and $\widetilde{s n}$ define the exact rotation

$$
J_{m}=\left[\begin{array}{cc}
\widetilde{c s} & \widetilde{s n} \\
-\widetilde{s n} & \widetilde{c s}
\end{array}\right]
$$

which takes $G_{m}+\delta G_{m}$ to $G_{m+1}$ :

$$
\left(G_{m}+\delta G_{m}\right) J_{m}=G_{m+1} .
$$

Let $G_{k i}^{\prime}$ and $G_{k j}^{\prime}$ be the new values for these entries computed by the algorithm. We have

$$
\begin{aligned}
\bar{G}_{k i}^{\prime} & =f l\left(\bar{G}_{k i}+\beta \bar{G}_{k j}\right)=\left(1+\varepsilon_{1}\right) \bar{G}_{k i}+\left(1+\varepsilon_{2}\right)\left(1+\varepsilon_{3}\right)\left(1+\varepsilon_{\beta}\right) \widetilde{\beta} \bar{G}_{k j} \\
& =\bar{G}_{k i}+\widetilde{\beta} \bar{G}_{k j}+\varepsilon_{1} \bar{G}_{k i}+\left(\varepsilon_{2}+\varepsilon_{3}+\varepsilon_{\beta}\right) \widetilde{\beta} \bar{G}_{k j} \\
\bar{D}_{i}^{\prime} & =f l\left(\bar{D}_{i} \widetilde{c s}\right)=\bar{D}_{i} \widetilde{c s}+\left(\varepsilon_{4}+\varepsilon_{c s}\right) \bar{D}_{i} \widetilde{c s}
\end{aligned}
$$

Using

$$
G_{\cdot i}=\bar{G}_{\cdot i} \bar{D}_{i}, \quad G_{\cdot i}^{\prime}=\bar{G}_{\cdot i}^{\prime} \bar{D}_{i}^{\prime},
$$

and (3.4.6), and ignoring the relative error of $O(\varepsilon)$, we obtain

$$
G_{\cdot i}^{\prime}=\widetilde{c s} G_{\cdot i}-\widetilde{s n} G_{\cdot j}+E_{\cdot i}
$$


where

$$
\left\|E_{. i}\right\|_{2} \leq\left(5 \widetilde{c s}\left\|G_{\cdot i}\right\|_{2}+8|\widetilde{s n}|\left\|G_{\cdot j}\right\|_{2}\right) \varepsilon .
$$

Here $G_{. i}$ refers to the $i$-th column of $G$, etc. Similarly,

$$
G_{\cdot j}^{\prime}=\widetilde{s n} G_{\cdot i}+\widetilde{c s} G_{\cdot j}+E_{\cdot j}
$$

where

$$
\left\|E_{\cdot j}\right\|_{2} \leq\left(8|\widetilde{s n}|\left\|G_{\cdot i}\right\|_{2}+5 \widetilde{c s}\left\|G_{\cdot j}\right\|_{2}\right) \varepsilon .
$$

Now (3.3.7) holds with

$$
\begin{aligned}
\left\|F_{\cdot i}\right\|_{2} & \leq \widetilde{c s}\left\|E_{\cdot i}\right\|_{2}+|\widetilde{s n}|\left\|E_{\cdot j}\right\|_{2} \\
& \leq \frac{1}{1+t^{2}}\left(5+8 t^{2}+13|t| x\right) d_{i} \varepsilon \\
\left\|F_{\cdot j}\right\|_{2} & \leq|\widetilde{s n}|\left\|E_{\cdot i}\right\|_{2}+\widetilde{c s}\left\|E_{\cdot j}\right\|_{2} \\
& \leq \frac{1}{1+t^{2}}\left(5+8 t^{2}+13|t| / x\right) d_{j} \varepsilon .
\end{aligned}
$$

We consider two cases, $x<\bar{x} \equiv 0.51$, and $x \geq \bar{x}$. First consider $x<\bar{x}$. Inserting $\bar{x}$ for $x$ in (3.4.7) we obtain

$$
\left\|F_{\cdot i}\right\|_{2} \leq 9.82 d_{i} \varepsilon .
$$

Inserting (3.3.10) into (3.4.8) we obtain

$$
\left\|F_{\cdot j}\right\|_{2} \leq 22.57 d_{j} \varepsilon
$$

and

$$
\left\|B_{m}\right\|_{2} \leq 33 \varepsilon .
$$

Now consider the case $x \geq \bar{x}$. Inserting 1 for $x$ in (3.4.7) we obtain

$$
\left\|F_{. i}\right\|_{2} \leq 13 d_{i} \varepsilon
$$

Inserting $1 / \bar{x}$ for $1 / x$ in $(3.4 .8)$, we obtain

$$
\left\|F_{\cdot j}\right\|_{2} \leq 19.25 d_{j} \varepsilon,
$$

so that (3.4.9) holds again. $æ$ 
Hyperbolic case. The proof is a combination of the above proof for the trigonometric case and the proof for the hyperbolic case of Th. 3.3.3. We denote the quantities $c s, s n$ and $s n 1=s n$ computed by Alg. 3.4.1 with $c h$ and $s h$, respectively. Let

$$
\begin{aligned}
\widetilde{c h} & \equiv 1 / \sqrt{1-t^{2}}, & \widetilde{s h} \equiv t / \sqrt{1-t^{2}} \\
\widetilde{\alpha} & \equiv t \bar{D}_{i} / \bar{D}_{j}, & \widetilde{\beta} \equiv t \bar{D}_{j} / D_{i} .
\end{aligned}
$$

For the calculated transformation parameters we have

$$
\begin{aligned}
c h & =\left(1+\varepsilon_{c h}\right) \widetilde{c h}, \\
\alpha & =\left(1+\varepsilon_{\alpha}\right) \widetilde{\alpha}, \quad \beta=\left(1+\varepsilon_{\beta}\right) \widetilde{\beta}, \quad\left|\varepsilon_{\alpha}\right|,\left|\varepsilon_{\beta}\right| \leq 2 \varepsilon .
\end{aligned}
$$

$\widetilde{c h}$ and $\widetilde{s h}$ define the exact rotation

$$
J_{m}=\left[\begin{array}{cc}
\widetilde{c h} & \widetilde{s h} \\
\widetilde{s h} & \widetilde{c h}
\end{array}\right]
$$

which takes $G_{m}+\delta G_{m}$ to $G_{m+1}$ :

$$
\left(G_{m}+\delta G_{m}\right) J_{m}=G_{m+1}
$$

Let $G_{k i}^{\prime}$ and $G_{k j}^{\prime}$ be the new values for these entries computed by the algorithm. As in the proof for the trigonometric case, we obtain

$$
G_{\cdot i}^{\prime}=\widetilde{\operatorname{ch}} G_{\cdot i}+\widetilde{s h} G_{\cdot j}+E_{\cdot i}
$$

where

$$
\left\|E_{\cdot i}\right\|_{2} \leq\left(2 \varepsilon+\left|\varepsilon_{c h}\right|\right) \widetilde{c h}\left\|G_{\cdot i}\right\|_{2}+\left(5 \varepsilon+\left|\varepsilon_{c h}\right|\right)|\widetilde{s h}|\left\|G_{\cdot j}\right\|_{2}
$$

and

$$
G_{\cdot j}^{\prime}=\widetilde{s h} G_{\cdot i}+\widetilde{c h} G_{\cdot j}+E_{\cdot j}
$$

where

$$
\left\|E_{\cdot j}\right\|_{2} \leq\left(5 \varepsilon+\left|\varepsilon_{c h}\right|\right)|\widetilde{s h}|\left\|G_{\cdot i}\right\|_{2}+\left(2 \varepsilon+\left|\varepsilon_{c h}\right|\right) \widetilde{c h}\left\|G_{\cdot j}\right\|_{2} .
$$

Now (3.3.15) holds with

$$
\begin{aligned}
& \left\|F_{\cdot i}\right\|_{2} \leq\left(\left(2 \varepsilon+\left|\varepsilon_{c h}\right|\right) \widetilde{c h}^{2}+\left(5 \varepsilon+\left|\varepsilon_{c h}\right|\right) \widetilde{s h}^{2}+\left(7 \varepsilon+2\left|\varepsilon_{c h}\right|\right) \widetilde{c h}|\widetilde{s h}| x\right) d_{i} \\
& \left\|F_{\cdot j}\right\|_{2} \leq\left(\left(2 \varepsilon+\left|\varepsilon_{c h}\right|\right) \widetilde{c h}^{2}+\left(5 \varepsilon+\left|\varepsilon_{c h}\right|\right) \widetilde{s h}^{2}+\left(7 \varepsilon+2\left|\varepsilon_{c h}\right|\right) \widetilde{c h}|\widetilde{s h}| / x\right) d_{j}
\end{aligned}
$$

As in Th. 3.3.3 we consider two cases, $|\zeta| \leq 3 /(2 \sqrt{2})$ and $|\zeta|>3 /(2 \sqrt{2})$. First consider $|\zeta| \leq 3 /(2 \sqrt{2})$. Then (3.2.18) and (3.2.20) hold, and

$$
\left|\varepsilon_{c h}\right| \leq\left(\frac{3}{8} \sqrt{\kappa}+\frac{7}{3}\right) \varepsilon
$$


The assertion of the theorem now follows by inserting $1 / x \leq \sqrt{2},(3.2 .20)$, and the above relation into (3.4.11).

Now consider $|\zeta|>3 /(2 \sqrt{2})$. Then the relations (3.2.23) hold for $t$, $\widetilde{c h}$ and $\widetilde{s h}$ with a relative error of $O(\varepsilon)$, and

$$
\left|\varepsilon_{c h}\right| \leq 4 \varepsilon
$$

We have two subcases, $x \geq 1 / \sqrt{2}$ and $x<1 / \sqrt{2}$. If $x \geq 1 / \sqrt{2}$, then the assertion of the theorem follows by inserting $1 / x \leq \sqrt{2}$, (3.4.12), and (3.2.23) into (3.4.11).

If $x<1 / \sqrt{2}$, then (3.3.19) holds, and the assertion of the theorem follows by inserting (3.3.19), (3.4.12), and (3.2.23) into (3.4.11).

Q.E.D.

Corollary 3.4.3 Assume Algorithm 3.4.1 converges, and that $G_{M}, J \equiv \bar{D}_{M} \bar{G}_{M}, J$ is the final pair which satisfies the stopping criterion. For $0 \leq m \leq M$ write $G_{m}=$ $B_{m} D_{m}$ with $D_{m}$ diagonal and $B_{m}$ with unit columns.

Let $\lambda_{j}$ be the $j$-th non-zero eigenvalue of $G_{0} J G_{0}^{T}$, and let $\lambda_{j}^{\prime}$ be the $j$-th computed eigenvalue. Then, with the relative error of $O(\varepsilon)$,

$$
(1-\gamma)^{2} \leq \frac{\lambda_{j}^{\prime}}{\lambda_{j}} \leq(1+\gamma)^{2}
$$

where

$$
\gamma=\varepsilon \sum_{m=0}^{M-1} \frac{C_{m}}{\sigma_{\min }\left(B_{m}\right)}+n \cdot \operatorname{tol} / 2+r(n+2) \varepsilon / 2 .
$$

Proof. See the proof of Cor. 3.3.4. The $r(n+2) \varepsilon / 2$ term comes from the facts that $c / \sqrt{a b}$ in the stopping criterion may now be underestimated by as much as $(n+2) \varepsilon$, and that the squares of the norms of the columns of $G_{M}$ are computed with a relative error not greater than $(n+2) \varepsilon$.

Q.E.D.

As in Subsect. 3.2.1, we can modify the fast implicit $J$-orthogonal Jacobi method in order to avoid potentially large $C_{m}$ in Th. 3.4 .2 in the hyperbolic case for $|\zeta| \leq$ $3 /(2 \sqrt{2})$. The algorithm of the modified method is obtained by combining Algorithms 3.4.1 and 3.2.7 in the obvious manner. We have the following:

Theorem 3.4.4 Let $G_{m}$ be the sequence of matrices generated by the modified fast implicit J-orthogonal Jacobi method in floating-point arithmetic with precision $\varepsilon$. Then Theorem 3.4.2 holds except that in the hyperbolic case for $|\zeta| \leq 3 /(2 \sqrt{2})$ the value $C_{m}$ is changed to $C_{m}=55$. Corollary 3.4.3 holds with this exception, too.

Proof. See the proof of Th. 3.3.6.

Q.E.D. 
As in Subsect. 3.3.1, we can keep the diagonal of the matrix $G^{T} G$ in a separate vector, thus saving $2(n+1)$ multiplications and $2(n-1)$ additions in every step. This is done as in Alg. 3.3.7, except that $\Delta_{i}$ 's are now refreshed using $\bar{G}_{m}$ and $\bar{D}_{m}$. All remarks about Alg. 3.3.7 from Subsect. 3.3.1 hold here, as well.

æ 


\subsubsection{Self-scaling rotations}

Analysing the fast rotation formulae (3.4.3) and (3.4.4), we see that these rotations make both values $\bar{D}_{i}$ and $\bar{D}_{j}$ smaller or larger, respectively. This can lead to underflow/overflow in some $\bar{D}_{i}$ during floating-point computation. As already mentioned, the probability that this happens is in the case of transition from the matrix $H=G J G^{T}$ to the pair $G, J$ very low. The probability of underflow/overflow can further be reduced by using self-scaling rotations suggested by Anda and Park [1]. The main idea is to "push" the diagonal element of $\bar{D}$ which is further away from 1 towards 1 . We use the "two way branch algorithm" of [1] and generalize it to the hyperbolic case. This adds four new fast rotations to the already existing ones (3.4.3) and (3.4.4). In this subsection we define these rotations, give the algorithm of the method, and present the error analysis.

The trigonometric self-scaling rotations from [1] are the following: supose that (3.4.2) holds. Simple calculation shows that either

$$
\left[\begin{array}{ll}
G_{\cdot i}^{\prime} & G_{\cdot j}^{\prime}
\end{array}\right]=\left[\begin{array}{ll}
\bar{G}_{\cdot i} & \bar{G}_{\cdot j}
\end{array}\right]\left[\begin{array}{ll}
1 & \alpha \\
0 & 1
\end{array}\right]\left[\begin{array}{ll}
1 & 0 \\
\beta & 1
\end{array}\right]\left[\begin{array}{cc}
\bar{D}_{i}^{\prime} & \\
& \bar{D}_{j}^{\prime}
\end{array}\right],
$$

where

$$
\begin{aligned}
& \alpha=\frac{\bar{D}_{i}}{\bar{D}_{j}} t, \quad \beta=-\frac{\bar{D}_{j}}{\bar{D}_{i}} c s \cdot s n, \quad t=s n / c s, \\
& \bar{D}_{i}^{\prime}=\bar{D}_{i} / c s, \quad \bar{D}_{j}^{\prime}=\bar{D}_{j} c s,
\end{aligned}
$$

or

$$
\left[\begin{array}{ll}
G_{\cdot i}^{\prime} & G_{\cdot j}^{\prime}
\end{array}\right]=\left[\begin{array}{ll}
\bar{G}_{\cdot i} & \bar{G}_{\cdot j}
\end{array}\right]\left[\begin{array}{ll}
1 & 0 \\
\beta & 1
\end{array}\right]\left[\begin{array}{ll}
1 & \alpha \\
0 & 1
\end{array}\right]\left[\begin{array}{cc}
\bar{D}_{i}^{\prime} & \\
& \bar{D}_{j}^{\prime}
\end{array}\right]
$$

where

$$
\begin{array}{ll}
\beta=-\frac{\bar{D}_{j}}{\bar{D}_{i}} t, & \alpha=\frac{\bar{D}_{i}}{\bar{D}_{j}} c s \cdot s n, \quad t=s n / c s, \\
\bar{D}_{i}^{\prime}=\bar{D}_{i} c s, & \bar{D}_{j}^{\prime}=\bar{D}_{j} / c s .
\end{array}
$$

The hyperbolic versions of the above rotations are either (3.4.14) with

$$
\begin{aligned}
& \alpha=\frac{\bar{D}_{i}}{\bar{D}_{j}} t, \quad \beta=\frac{\bar{D}_{j}}{\bar{D}_{i}} \text { ch } \cdot s h, \quad t=s h / c h, \\
& \bar{D}_{i}^{\prime}=\bar{D}_{i} / c h, \quad \bar{D}_{j}^{\prime}=\bar{D}_{j} c h,
\end{aligned}
$$

or (3.4.16) with

$$
\begin{aligned}
& \beta=\frac{\bar{D}_{j}}{\bar{D}_{i}} t, \quad \alpha=\frac{\bar{D}_{i}}{\bar{D}_{j}} \text { ch } \cdot \text { sh }, \quad t=s h / c h, \\
& \bar{D}_{i}^{\prime}=\bar{D}_{i} \text { ch }, \quad \bar{D}_{j}^{\prime}=\bar{D}_{j} / \text { ch } .
\end{aligned}
$$


The rotation (3.4.3) makes both $\bar{D}_{i}$ and $\bar{D}_{j}$ smaller. We use it in the trigonometric case when $\bar{D}_{i}, \bar{D}_{j} \geq 1$. The rotation (3.4.4) makes both $\bar{D}_{i}$ and $\bar{D}_{j}$ larger. We use it in the hyperbolic case when $\bar{D}_{i}, \bar{D}_{j}<1$.

The rotations (3.4.14), (3.4.15) and (3.4.16), (3.4.19) make $\bar{D}_{i}$ larger and $\bar{D}_{j}$ smaller so they are always used when $\bar{D}_{i}<1 \leq \bar{D}_{j}$. The first is also used in the trigonometric case when $\bar{D}_{i} \leq \bar{D}_{j}<1$ and the second is used in the hyperbolic case when $1 \leq \bar{D}_{i} \leq \bar{D}_{j}$.

The rotations (3.4.16), (3.4.17) and (3.4.14), (3.4.18) make $\bar{D}_{i}$ smaller and $\bar{D}_{j}$ larger so they are always used when $\bar{D}_{i} \geq 1>\bar{D}_{j}$. The first is also used in the trigonometric case when $1>\bar{D}_{i}>\bar{D}_{j}$, and the second is used in the hyperbolic case when $\bar{D}_{i}>\bar{D}_{j} \geq 1$.

Thus, we have the following

Algorithm 3.4.5 Fast implicit J-orthogonal Jacobi method with self-scaling rotations for the pair $G, J$. tol is a user defined stopping criterion.

for $k=1$ to $r$

$$
D_{k}=1
$$

endfor

repeat

for all pairs $i<j$

$$
\begin{aligned}
& \text { /* compute }\left[\begin{array}{ll}
a & c \\
c & b
\end{array}\right] \equiv \text { the }(i, j) \text { submatrix of } G^{T} G^{*} / \\
& \qquad \begin{array}{l}
a \\
\text { a } \sum_{k=1}^{n} G_{k i}^{2} \\
b=D_{j}^{2} \sum_{k=1}^{n} G_{k j}^{2} \\
c=D_{i} D_{j} \sum_{k=1}^{n} G_{k i} * G_{k j}
\end{array}
\end{aligned}
$$

${ }^{*}$ compute the parameter hyp: hyp $=1$ for the hyperbolic and

hyp $=-1$ for the trigonometric rotation, respectively */

if $1 \leq i \leq$ npos $<j \leq r$ then

$$
\text { hyp }=1
$$

else

$$
\text { hyp }=-1
$$

endif

${ }^{*}$ compute the $J$-orthogonal Jacobi rotation which diagonalizes

$$
\begin{aligned}
& \quad\left[\begin{array}{ll}
H_{i i} & H_{i j} \\
H_{j i} & H_{j j}
\end{array}\right] \equiv\left[\begin{array}{ll}
a & c \\
c & b
\end{array}\right] * / \\
& \zeta=-h y p *(b+h y p * a) /(2 c) \\
& t=\operatorname{sign}(\zeta) /\left(|\zeta|+\sqrt{\zeta^{2}-h y p}\right) \\
& h=\sqrt{1-h y p * t^{2}} \\
& c s=1 / h \\
& \text { sn }=t / h \\
& /^{*} \text { update columns } i \text { and } j \text { of } G \text { and } D_{i} \text { and } D_{j}^{*} / \\
& \text { if (hyp } \left.=1 \text { and } D_{i}, D_{j}<1\right) \text { or }\left(h y p=-1 \text { and } D_{i}, D_{j} \geq 1\right) \text { then }
\end{aligned}
$$




$$
\begin{aligned}
& \alpha=t * D_{i} / D_{j} \\
& \beta=h y p * t * D_{j} / D_{i} \\
& \text { for } k=1 \text { to } n \\
& \quad \text { tmp }=G_{k i} \\
& \quad G_{k i}=\text { tmp }+\alpha * G_{k j} \\
& \quad G_{k j}=\beta * t m p+G_{k j} \\
& \text { endfor } \\
& D_{i}=D_{i} * c s \\
& D_{j}=D_{j} * c s
\end{aligned}
$$

elseif (hyp $=1$ and $\left(D_{j}<1 \leq D_{i}\right.$ or $\left.D_{i}>D_{j} \geq 1\right)$ ) or

(hyp $=-1$ and $\left(D_{i}<1 \leq D_{j}\right.$ or $\left.D_{i} \leq D_{j} \leq 1\right)$ ) then

$\alpha=t * D_{i} / D_{j}$

$\beta=h y p * c s * s n * D_{j} / D_{i}$

for $k=1$ to $n$

$$
\begin{aligned}
& G_{k j}=\alpha * G_{k i}+G_{k j} \\
& G_{k i}=G_{k i}+\beta * G_{k j}
\end{aligned}
$$

endfor

$D_{i}=D_{i} / c s$

$D_{j}=D_{j} * c s$

else

$$
\begin{aligned}
& \beta=\text { hyp } * t * D_{j} / D_{i} \\
& \alpha=c s * \text { sn } * D_{i} / D_{j} \\
& \text { for } k=1 \text { to } n \\
& \qquad \begin{array}{l}
G_{k i}=G_{k i}+\beta * G_{k j} \\
G_{k j}=\alpha * G_{k i}+G_{k j}
\end{array}
\end{aligned}
$$

endfor

$$
D_{i}=D_{i} * c s
$$

endif

$D_{j}=D_{j} / c s$

endfor

until convergence $($ all $|c| / \sqrt{a b} \leq$ tol $)$

${ }^{*}$ the computed non-zero eigenvalues of $H=G J G^{T}$ (and of the pair $G^{T} G, J$ ) are

$$
\lambda_{j}=\left(\sum_{k=1}^{n} G_{k j}^{2}\right) D_{j}^{2} J_{j j}^{*} /
$$

${ }^{*}$ the computed eigenvectors of $H$ are the normalized columns of the final $G * /$

The version of the algorithm where the diagonal of $G^{T} G$ is kept in a separate vector is obtained by combining Algorithms 3.4.5 and 3.3.7. The only exception from Alg. 3.3.7 is that $\Delta_{i}$ 's are refreshed using $\bar{G}_{m}$ and $\bar{D}_{m}$. Further, the modified method is obtained by combining Algorithms 3.4.5 and 3.2.7. Error analysis of the self-scaling rotations is similar to the analysis of the fast rotations from previous section. The following theorem gives error analysis of the modified method: 
Theorem 3.4.6 Let $\bar{G}_{m}, \bar{D}_{m}$ be the sequences of matrices generated by the modified fast implicit J-orthogonal Jacobi algorithm with self-scaling rotations in floatingpoint arithmetic with precision $\varepsilon$; that is $\bar{G}_{m+1}$ is obtained from $\bar{G}_{m}$ by applying one of the fast rotations, and $D_{m+1}$ is obtained from $D_{m}$ by one of the formulae (3.4.3), (3.4.4), (3.4.15), (3.4.17-3.4.19). Then Th. 3.4.2 holds with

$$
C_{m}=191
$$

in all cases. Corollary 3.4.3 holds as well.

Proof. For the standard fast rotations (3.4.3) and (3.4.4), the theorem follows from Theorems 3.4.2 and 3.4.4.

Suppose that we apply the hyperbolic self-scaling rotation defined with (3.4.16) and (3.4.19). Let the quantities $c s$ and $s n$ computed by Alg. 3.4.5 be denoted by $c h$ and $s h$, respectively. Let

$$
\begin{aligned}
\widetilde{c h} & \equiv 1 / \sqrt{1-t^{2}}, & \widetilde{s h} \equiv t / \sqrt{1-t^{2}} \\
\widetilde{\beta} & \equiv t \bar{D}_{j} / \bar{D}_{i}, & \widetilde{\alpha} \equiv \bar{D}_{i} / \bar{D}_{j} \widetilde{c h} \cdot \widetilde{s h} .
\end{aligned}
$$

Since we are using the modified method, the relations (3.2.23) always hold, and for the calculated transformation parameters we have

$$
\begin{aligned}
& c h=\left(1+\varepsilon_{c h}\right) \widetilde{c h}, \quad s h=\left(1+\varepsilon_{s h}\right) \widetilde{s h}, \quad\left|\varepsilon_{c h}\right|,\left|\varepsilon_{s h}\right| \leq 4 \varepsilon \\
& \beta=\left(1+\varepsilon_{\beta}\right) \widetilde{\beta}, \quad\left|\varepsilon_{\beta}\right| \leq 2 \varepsilon . \\
& \alpha=\left(1+\varepsilon_{\alpha}\right) \tilde{\alpha}, \quad\left|\varepsilon_{\alpha}\right| \leq 11 \varepsilon,
\end{aligned}
$$

$\widetilde{c h}$ and $\widetilde{s h}$ define the exact rotation

$$
J_{m}=\left[\begin{array}{ll}
\widetilde{c h} & \widetilde{s h} \\
\widetilde{s h} & \widetilde{c h}
\end{array}\right]
$$

which takes $G_{m}+\delta G_{m}$ to $G_{m+1}$, i.e. $\left(G_{m}+\delta G_{m}\right) J_{m}=G_{m+1}$. Let $G_{k i}^{\prime}$ and $G_{k j}^{\prime}$ be the new values for these entries computed by the algorithm. From Alg. 3.4.5 we have

$$
\begin{aligned}
\bar{G}_{k i}^{\prime} & =f l\left(\bar{G}_{k i}+\beta \bar{G}_{k j}\right)=\left(1+\varepsilon_{1}\right) \bar{G}_{k i}+\left(1+\varepsilon_{2}\right)\left(1+\varepsilon_{3}\right)\left(1+\varepsilon_{\beta}\right) \widetilde{\beta} \bar{G}_{k j} \\
& =\bar{G}_{k i}+\widetilde{\beta} \bar{G}_{k j}+\varepsilon_{1} \bar{G}_{k i}+\left(\varepsilon_{2}+\varepsilon_{3}+\varepsilon_{\beta}\right) \widetilde{\beta} \bar{G}_{k j} \\
\bar{D}_{i}^{\prime} & =f l\left(\bar{D}_{j} c h\right)=\bar{D}_{i} \widetilde{c h}+\left(\varepsilon_{4}+\varepsilon_{c h}\right) \bar{D}_{i} \widetilde{c h}
\end{aligned}
$$

Using

$$
G_{\cdot j}=\bar{G}_{\cdot j} \bar{D}_{j}, \quad G_{\cdot j}^{\prime}=\bar{G}_{\cdot j}^{\prime} \bar{D}_{j}^{\prime},
$$

and (3.4.21), we obtain

$$
G_{\cdot i}^{\prime}=\widetilde{\operatorname{ch}} G_{\cdot i}+\widetilde{s h} G_{\cdot j}+E_{\cdot i}
$$


where

$$
\left\|E_{\cdot i}\right\|_{2} \leq\left(\widetilde{6 c h}\left\|G_{\cdot i}\right\|_{2}+9|\widetilde{s h}|\left\|G_{\cdot j}\right\|_{2}\right) \varepsilon
$$

Further,

$$
\begin{aligned}
\bar{G}_{k j}^{\prime} & =f l\left(\alpha \bar{G}_{k i}^{\prime}+\bar{G}_{k j}\right)=\left(1+\varepsilon_{5}\right)\left(1+\varepsilon_{6}\right)\left(1+\varepsilon_{\alpha}\right) \widetilde{\alpha} \bar{G}_{k i}^{\prime}+\left(1+\varepsilon_{7}\right) \bar{G}_{k j} \\
\bar{D}_{j}^{\prime} & =f l\left(\bar{D}_{j} / c h\right)=\bar{D}_{j} / c h+\left(\varepsilon_{8}+\varepsilon_{c h}^{\prime}\right) \bar{D}_{j} / c h
\end{aligned}
$$

where $\left|\varepsilon_{c h}^{\prime}\right| \leq 4 \varepsilon$, so that

$$
G_{\cdot j}^{\prime}=\bar{G}_{\cdot j}^{\prime} \bar{D}_{j}^{\prime}=\widetilde{s h} G_{\cdot i}+\widetilde{c h} G_{\cdot j}+E_{\cdot j}
$$

where

$$
\left\|E_{\cdot j}\right\|_{2} \leq\left(19|\widetilde{s h}|\left\|G_{\cdot i}\right\|_{2}+\left(5 \widetilde{c h}+\frac{1}{\widetilde{c h}}+17 \frac{\widetilde{s h}^{2}}{\widetilde{c h}}\right)\right) \varepsilon
$$

Now (3.3.15) holds with

$$
\begin{aligned}
\left\|F_{\cdot i}\right\|_{2} & \leq \widetilde{c h}\left\|E_{. i}\right\|_{2}+|\widetilde{s h}|\left\|E_{\cdot j}\right\|_{2} \\
& \leq\left(6 \widetilde{c h}^{2}+19 \widetilde{s h}^{2}+\left(14 \widetilde{c h}|\widetilde{s h}|+17|t| \widetilde{s h}^{2}+|t|\right) x\right) \varepsilon d_{i}, \\
\left\|F_{\cdot j}\right\|_{2} & \leq|\widetilde{s h}| \cdot\left\|E_{. i}\right\|_{2}+\widetilde{c h}\left\|E_{\cdot j}\right\|_{2} \\
& \leq\left(25 \widetilde{c h}|\widetilde{s h}| / x+26 \widetilde{s h}^{2}+5 \widetilde{c h}^{2}+1\right) \varepsilon d_{j} .
\end{aligned}
$$

Here

$$
\left\|G_{\cdot i}\right\|_{2} \equiv d_{i}, \quad\left\|G_{\cdot j}\right\|_{2} \equiv d_{j}, \quad, \quad x \equiv d_{j} / d_{i} .
$$

We consider two cases, $x \geq 1 / \sqrt{2}$ and $x<1 / \sqrt{2}$. If $x \geq 1 / \sqrt{2}$, then by inserting $1 / x \leq \sqrt{2}$ and (3.2.23) into (3.4.22) we have

$$
\begin{aligned}
\left\|F_{\cdot i}\right\|_{2} & \leq 64 d_{i} \varepsilon, \quad\left\|F_{\cdot j}\right\|_{2} \leq 86 d_{j} \varepsilon \\
\left\|\delta B_{m}\right\|_{2} & \leq\left\|F_{\cdot i}\right\|_{2} / d_{i}+\left\|F_{\cdot j}\right\|_{2} / d_{j} \leq 150 \varepsilon
\end{aligned}
$$

If $x<1 / \sqrt{2}$, then (3.3.19) holds, and by inserting (3.3.19), and (3.2.23) into (3.4.22) we have

$$
\begin{aligned}
\left\|F_{\cdot i}\right\|_{2} & \leq 54 d_{i} \varepsilon, \quad\left\|F_{\cdot j}\right\|_{2} \leq 137 d_{j} \varepsilon, \\
\left\|\delta B_{m}\right\|_{2} & \leq 191 \varepsilon .
\end{aligned}
$$

The analysis of the three remaining types of the self-scaling rotations is similar. Q.E.D.

æ 


\section{Chapter 4}

\section{Symmetric indefinite decomposition}

\subsection{Introduction and algorithm}

In order to solve the eigenvalue problem

$$
H x=\lambda x, \quad x \neq 0
$$

where $H$ is a $n \times n$ real symmetric matrix with $\operatorname{rank}(H)=r \leq n$, by any of the implicit (one-sided) Jacobi methods of Chap. 3 for which we have good error bounds, we first decompose $H$ as

$$
\text { PHP } P^{T}=G J G^{T}, \quad J=I_{n p o s} \oplus-I_{r-\text { npos }} .
$$

Here $G$ is a $n \times r$ matrix (i.e. $G$ has full column rank), $P$ is a permutation matrix, and npos is the number of positive eigenvalues of $H$. The decomposition (1.1) is then obtained by multiplying (4.1.2) by $P^{T}$ from the left and $P$ from the right, that is, the implicit Jacobi is applied to the pair $P^{T} G, J$.

The chapter is organized as follows: in this section we give the algorithm of the symmetric indefinite decomposition (4.1.2). In Sect. 4.2 we give the error analysis of the method. In Sect. 4.3 we give the final error bounds for the computed eigensolution of the symmetric eigenvalue problem. Finally, in Sect. 4.4 we show an interesting fact that the scaled condition of the matrix $G^{T} G$ is bounded by a function of $n$ irrespectively of the condition of the starting matrix $H$.

We now give the algorithm of the symmetric indefinite decomposition (4.1.2). Our method is essentially the method of Bunch and Parlett [6] with some modifications. The method of Bunch and Parlett decomposes $H$ as

$$
P H P^{T}=L T L^{T}
$$


where $L$ is lower triangular matrix with unit diagonal, and $T$ is block diagonal matrix with $(1 \times 1)$ and $(2 \times 2)$ blocks. We shortly describe one step of the algorithm. Let $\bar{P}$ be a permutation matrix such that

$$
\bar{P} H \bar{P}^{T}=\left[\begin{array}{cc}
X & C^{T} \\
C & Y
\end{array}\right]
$$

where $X$ is nonsingular $k \times k$ matrix, $k \in\{1,2\}, C$ is a $(n-k) \times k$ matrix, and $Y$ is a $(n-k) \times(n-k)$ matrix. Such $\bar{P}$ always exists because $H$ is nonsingular. We can decompose $\bar{P} H \bar{P}^{T}$ as

$$
\begin{aligned}
\bar{P} H \bar{P}^{T} & =\bar{L}\left[\begin{array}{cc}
X & 0 \\
0 & H_{1}
\end{array}\right] \bar{L}^{T}, \\
\bar{L} & =\left[\begin{array}{cc}
I_{k} & 0 \\
C X^{-1} & I_{n-k}
\end{array}\right], \\
H_{1} & =Y-C X^{-1} C^{T} .
\end{aligned}
$$

Recursive application of (4.1.5) yields (4.1.3) in the obvious manner. We choose $1 \times 1$ or $2 \times 2$ pivot according to the unequilibrated diagonal pivoting from [6] ${ }^{1}$ : set

$$
\alpha=(1+\sqrt{17}) / 8
$$

and calculate

$$
\nu_{0}=\max _{i \neq j}\left|H_{i j}\right|, \quad \nu_{1}=\max _{i}\left|H_{i i}\right| .
$$

We choose a $1 \times 1$ pivot if and only if $\nu_{1} \geq \alpha \nu_{0}$, and a $2 \times 2$ pivot otherwise. For a $1 \times 1$ pivot, we choose $\bar{P}$ in (4.1.4) to interchange row and column 1 with $s$, where $s$ is the least integer such that $\nu_{1}=\left|H_{s s}\right|$. Therefore, $|X|=\nu_{1}$. For a $2 \times 2$ pivot, we choose $\bar{P}$ to interchange rows and columns 1 with $q$ and 2 with $p$, where $q$ is the least column integer and $p$ is the least row integer in the $q$-th column such that $\nu_{0}=\left|H_{p q}\right|$ (note that $p>q$ ). Therefore,

$$
\begin{aligned}
\left|\left(\bar{P} H \bar{P}^{T}\right)_{21}\right| & =\nu_{0} \\
-\operatorname{det}(X) & =|\operatorname{det} X| \geq \nu_{0}^{2}-\nu_{1}^{2} .
\end{aligned}
$$

Bunch and Parlett [6] showed that the above choice of $\alpha$ minimizes the element growth which can take place in transition from $H$ to $H_{1}$, and that for any pivoting strategy which satisfies (4.1.7)

$$
\left|L_{i j}\right| \leq \begin{cases}1.562 & \text { if } L_{i j} \text { is obtained after a } 1 \times 1 \text { pivot } \\ 2.781 & \text { otherwise }\end{cases}
$$

\footnotetext{
${ }^{1}$ See Rem. 4.2.3.
} 
To obtain decomposition (4.1.2), we futher decompose $P H P^{T}$ as

$$
P H P^{T}=L Q Q^{T} T Q Q^{T} L^{T}
$$

where $Q$ is orthogonal block diagonal matrix with the same structure as $T$. The $1 \times 1$ blocks of $Q$ are 1 , and the $2 \times 2$ blocks of $Q$ are elementary orthogonal plane rotation matrices of the form

$$
\left[\begin{array}{cc}
c s & s n \\
-s n & c s
\end{array}\right], \quad c s^{2}+s n^{2}=1
$$

chosen to diagonalize corresponding $2 \times 2$ blocks of $T$. Denoting $L_{1}=L Q$ and $D_{1}=Q^{T} T Q$ we can write $P H P^{T}=L_{1} D_{1} L_{1}^{T}$, where $L_{1}$ is lower block triangular and $D_{1}$ is diagonal matrix. Due to (4.1.7) the $2 \times 2$ diagonal blocks of $D_{1}$ which correspond to $2 \times 2$ diagonal blocks of $T$ always have one positive and one negative element. Further we have

$$
P H P^{T}=L_{1} \sqrt{\left|D_{1}\right|} J_{1} \sqrt{\left|D_{1}\right|} L_{1}^{T},
$$

where $J_{1}$ is diagonal with $J_{1, i i} \in\{-1,1\}$. Finally,

$$
P H P^{T}=L_{1} \sqrt{\left|D_{1}\right|} P_{1} P_{1}^{T} J_{1} P_{1} P_{1}^{T} \sqrt{\left|D_{1}\right|} L_{1}^{T},
$$

where $P_{1}$ is a permutation matrix chosen to sort elements of $J_{1}$ according to the relation (4.1.2). Setting $G=L_{1} \sqrt{\left|D_{1}\right|} P_{1}$ and $J=P_{1}^{T} J_{1} P_{1}$ we obtain the decomposition (4.1.2).

If $H$ is positive definite, the above algorithm reduces to the Cholesky decomposition with complete pivoting (see e.g. [13]), that is

$$
P H P^{T}=L L^{T} \text {. }
$$

Combining (4.1.5) and (4.1.9), and using

$$
Q^{T} X Q=D
$$

where $D$ is a $1 \times 1$ or $2 \times 2$ diagonal matrix, we obtain (in the notation of (4.1.5))

$$
\begin{aligned}
\bar{P} H \bar{P}^{T} & =\bar{G}\left[\begin{array}{cc}
J & 0 \\
0 & H_{1}
\end{array}\right] \bar{G}^{T}, \\
\bar{G} & =\left[\begin{array}{cc}
B & 0 \\
Z & I
\end{array}\right] \\
B & =Q|D|^{1 / 2} \\
Z & =C Q|D|^{-1 / 2} J \\
H_{1} & =Y-Z J Z^{T} .
\end{aligned}
$$

Thus, we have the following: 


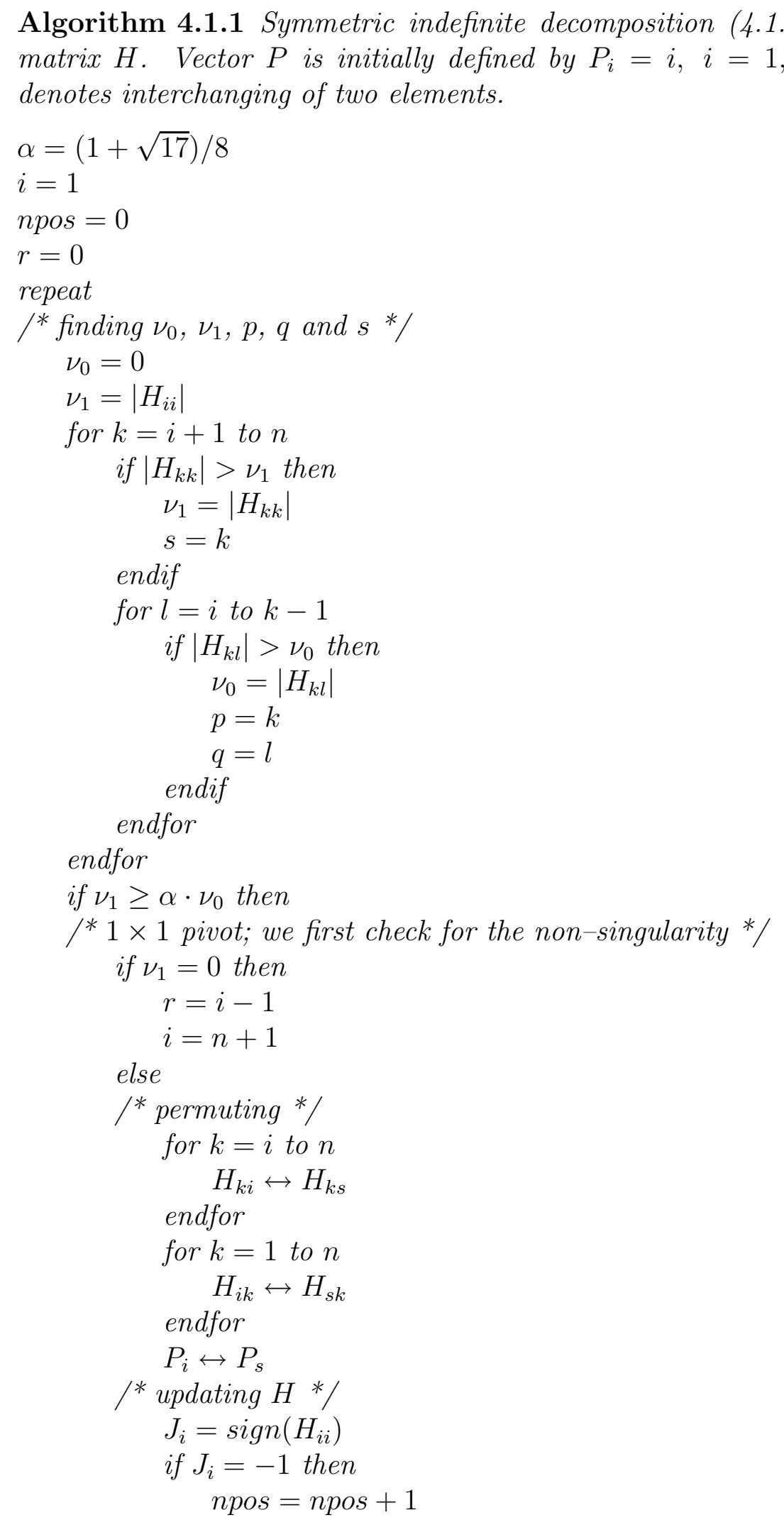




$$
\begin{gathered}
\text { endif } \\
\text { temp }=\sqrt{\left|H_{i i}\right|} \\
H_{i i}=\text { temp } \\
\text { for } k=i+1 \text { to } n \\
H_{k i}=H_{k i} * J_{i} / \text { temp } \\
H_{i k}=0 \\
\text { for } l=i+1 \text { to } k \\
H_{k l}=H_{k l}-H_{k i} * H_{l i} * J_{i} \\
H_{l k}=H_{k l} \\
\text { endfor } \\
\text { endfor } \\
i=i+1 \\
\text { endif } \quad \text { else } \quad \text { pivot; we first permute } * / \\
\text { F }_{2} \times 2 \text { po } n \\
\text { for } k=i \text { to } \\
H_{k i} \leftrightarrow H_{k q} \\
H_{k, i+1} \leftrightarrow H_{k p} \\
\text { endfor } \\
\text { for } k=1 \text { to } n \\
H_{i k} \leftrightarrow H_{q k} \\
H_{i+1, k} \leftrightarrow H_{p k} \\
\text { endfor } \\
P_{i} \leftrightarrow P_{q} \\
P_{i+1} \leftrightarrow P_{p}
\end{gathered}
$$

else

$I^{*}$ calculating the orthogonal matrix which diagonalizes $\left[\begin{array}{cc}H_{i i} & H_{i, i+1} \\ H_{i+1, i} & H_{i+1, i+1}\end{array}\right] * /$

$$
\begin{aligned}
& \zeta=\left(H_{i+1, i+1}-H_{i i}\right) /\left(2 * H_{i+1, i}\right) \\
& t=\operatorname{sign}(\zeta) /\left(|\zeta|+\sqrt{\zeta^{2}+1}\right)
\end{aligned}
$$$$
h=\sqrt{1+t^{2}}
$$

$c s=1 / h$

$s n=t / h$

/* updating $H^{*} /$

$$
\begin{aligned}
& a=H_{i i}-H_{i+1, i} * t \\
& b=H_{i+1, i+1}+H_{i+1, i} * t \\
& J_{i}=\operatorname{sign}(a) \\
& J_{i+1}=\operatorname{sign}(b) \\
& \text { npos }=\text { npos }+1 \\
& a=|a| \\
& b=|b| \\
& H_{i i}=c s * a \\
& H_{i, i+1}=\operatorname{sn} * b
\end{aligned}
$$




$$
\begin{aligned}
& H_{i+1, i}=-s n * a \\
& H_{i+1, i+1}=c s * b \\
& \text { for } k=i+2 \text { to } n \\
& \text { temp }=H_{k i} \\
& H_{k i}=\left(t e m p * c s-H_{k, i+1} * s n\right) * J_{i} / a \\
& H_{k, i+1}=\left(t e m p * s n+H_{k, i+1} * c s\right) * J_{i+1} / b \\
& H_{i k}=0 \\
& H_{i+1, k}=0 \\
& \text { for } l=i+2 \text { to } k \\
& H_{k l}=H_{k l}-H_{k i} * H_{l i} * J_{i}-H_{k, i+1} * H_{l, i+1} * J_{i+1} \\
& H_{l k}=H_{k l} \\
& \text { endfor } \\
& \text { endfor } \\
& i=i+2 \\
& \text { endif } \\
& \text { until } i>n \\
& { }^{*} \text { if non-singularity did not occur, then rank equals dimension */ } \\
& \text { if } r=0 \text { then } \\
& r=n \\
& \text { endif } \\
& { }^{*} \text { permuting the columns of } H \text { to sort } J \text { */ } \\
& k=\text { npos }+1 \\
& \text { for } l=1 \text { to npos } \\
& \text { if } J_{l}=-1 \text { then } \\
& \text { while } J_{k}=-1 \\
& k=k+1 \\
& \text { endwhile } \\
& \text { for } m=1 \text { to } n \\
& H_{m l} \leftrightarrow H_{m k} \\
& \text { endfor } \\
& k=k+1 \\
& \text { endif }
\end{aligned}
$$

æ 


\subsection{Error analysis}

In this section we give error analysis of the symmetric indefinite decomposition defined by Alg. 4.1.1. In our proof we use the approach from Th. 3.3.1 of [16]. We compare our result with the existing analysis of the algorithm of Bunch and Parlett [6] by Bunch [3].

Theorem 4.2.1 Let $G$ and $J$ be the factors of a real symmetric matrix $H$ computed by Alg. 4.1.1 in floating-point arithmetic with precision $\varepsilon$. Then, with the relative error of $O(\varepsilon), G$ and $J$ satisfy

$$
\begin{aligned}
G J G^{T} & =P H P^{T}+E \\
|E| & \leq 136 n\left(P|H| P^{T}+|G||G|^{T}\right) \varepsilon .
\end{aligned}
$$

Proof. The proof is by induction on $n$. The theorem obviously holds for all matrices of order 1 . To begin the induction, we must also analyse matrices of order 2 for a $2 \times 2$ pivot. Let

$$
\begin{aligned}
\widetilde{\zeta} & =\left(H_{22}-H_{11}\right) /\left(2 H_{21}\right) \\
\widetilde{t} & =\operatorname{sign}(\widetilde{\zeta}) /\left(|\widetilde{\zeta}|+\sqrt{1+\widetilde{\zeta}^{2}}\right) \\
\widetilde{c s} & =1 / \sqrt{1+\widetilde{t}^{2}} \\
\widetilde{s n} & =\widetilde{t} / \sqrt{1+\widetilde{t}^{2}} \\
\widetilde{a} & =H_{11}-H_{21} \widetilde{t} \\
\widetilde{b} & =H_{22}+H_{21} \widetilde{t}
\end{aligned}
$$

and $\widetilde{G}_{i j}$ denote the exact quantities computed by Alg. 4.1.1, i.e. without rounding errors. Since

$$
\left|H_{21}\right|=\nu_{0}, \quad \max \left\{\left|H_{11}\right|,\left|H_{22}\right|\right\} \leq \nu_{1},
$$

the fact that we perform a $2 \times 2$ step implies

$$
|\widetilde{\zeta}| \leq \begin{cases}\alpha & \text { if } \operatorname{sign}\left(H_{11}\right)=-\operatorname{sign}\left(H_{22}\right) \\ \alpha / 2 & \text { otherwise } .\end{cases}
$$

Now we show that the computed quantities $t, c s, s n, a$ and $b$ have small relative errors with respect to the exact quantities from (4.2.2). Single subscribed $\varepsilon$ 's denote quantities of absolute value less than or equal to $\varepsilon$. Most of the subsequent inequalities hold with a relative error of $O(\varepsilon)$. Using (4.2.3) and the maximum in (4.2.4), we have

$$
\begin{aligned}
\zeta & =f l\left(\frac{H_{22}-H_{11}}{2 H_{21}}\right)=\frac{H_{22}\left(1+\varepsilon_{1}\right)-H_{11}\left(1+\varepsilon_{2}\right)}{2 H_{21}\left(1+\varepsilon_{3}\right)}\left(1+\varepsilon_{4}\right) \\
& =\widetilde{\zeta}+\varepsilon_{\zeta}
\end{aligned}
$$


where $\left|\varepsilon_{\zeta}\right| \leq 3 \alpha \varepsilon$. This implies that the equality

$$
f l\left(1+\zeta^{2}\right)=\left(1+\varepsilon_{5}\right)\left(1+\left(\widetilde{\zeta}+\varepsilon_{\zeta}\right)^{2}\left(1+\varepsilon_{6}\right)\right)=\left(1+\widetilde{\zeta}^{2}\right)\left(1+\varepsilon^{\prime}\right),
$$

holds for some

$$
\left|\varepsilon^{\prime}\right| \leq 2\left|\varepsilon_{\zeta} \widetilde{\zeta}\right|+\left(\left|\varepsilon_{5}\right|+\left|\varepsilon_{6}\right|\right) \widetilde{\zeta}^{2}+\left|\varepsilon_{5}\right| \leq 4.3 \varepsilon
$$

Further, the equality

$$
\begin{aligned}
f l\left(|\zeta|+\sqrt{1+\zeta^{2}}\right) & =\left(1+\varepsilon_{7}\right)\left(\left|\widetilde{\zeta}+\varepsilon_{\zeta}\right|+\left(1+\varepsilon_{8}\right)\left(1+\varepsilon^{\prime} / 2\right) \sqrt{1+\widetilde{\zeta}^{2}}\right) \\
& =\left(1+\varepsilon^{\prime \prime}\right)\left(|\widetilde{\zeta}|+\sqrt{1+\widetilde{\zeta}^{2}}\right)
\end{aligned}
$$

holds for some

$$
\left|\varepsilon^{\prime \prime}\right| \leq\left|\varepsilon_{\zeta}\right|+\left|\varepsilon_{7}\right|(1+|\widetilde{\zeta}|)+\left|\varepsilon_{8}\right|+\left|\varepsilon^{\prime}\right| / 2 \leq 7 \varepsilon
$$

so that finally

$$
\begin{aligned}
t & =\widetilde{t}\left(1+\varepsilon_{t}\right), \quad\left|\varepsilon_{t}\right| \leq 8 \varepsilon, & & \\
c s & =f l\left(1 / \sqrt{1+t^{2}}\right)=\widetilde{c s}\left(1+\varepsilon_{c s}\right), & & \left|\varepsilon_{c s}\right| \leq 11 \varepsilon, \\
s n & =f l\left(t / \sqrt{1+t^{2}}\right)=\widetilde{s n}\left(1+\varepsilon_{s n}\right), & & \left|\varepsilon_{s n}\right| \leq 19 \varepsilon .
\end{aligned}
$$

Let

$$
\begin{aligned}
& a=f l\left(H_{11}-H_{21} t\right) \\
& b=f l\left(H_{22}+H_{21} t\right) .
\end{aligned}
$$

If $\operatorname{sign}\left(H_{11}\right)=-\operatorname{sign}\left(H_{22}\right)$, then $a$ and $b$ are both calculated by addition and have small relative errors, i.e.

$$
a=\widetilde{a}\left(1+\varepsilon_{a}\right), \quad b=\widetilde{b}\left(1+\varepsilon_{b}\right), \quad\left|\varepsilon_{a}\right|,\left|\varepsilon_{b}\right| \leq 10 \varepsilon .
$$

Let $\operatorname{sign}\left(H_{11}\right)=\operatorname{sign}\left(H_{22}\right)$. Assume further that $H_{11} \geq H_{22} \geq 0$. Then $a$ is again calculated by addition and (4.2.7) holds for it. Using (4.2.4), $\left|H_{21}\right|=\nu_{0}$, and $\left|H_{22}\right| \leq$ $\nu_{1}$, we have

$$
\begin{aligned}
|\widetilde{b}| & =\left|H_{22}+H_{21} \widetilde{t}\right| \geq|\widetilde{t}|\left|H_{21}\right|-\left|H_{22}\right| \\
& \geq \nu_{0}\left(\frac{1}{\alpha / 2+\sqrt{1+\alpha^{2} / 4}}-\alpha\right) \geq 0.088 \nu_{0} .
\end{aligned}
$$

Therefore,

$$
\begin{array}{r}
b=H_{22}\left(1+\varepsilon_{9}\right)+\left(1+\varepsilon_{10}\right)\left(1+\varepsilon_{11}\right)\left(1+\varepsilon_{t}\right) H_{21} \tilde{t}=\widetilde{b}\left(1+\varepsilon_{b}^{\prime}\right), \\
\left|\varepsilon_{b}^{\prime}\right| \leq\left(\left|H_{22} \varepsilon_{9}\right|+\left(\left|\varepsilon_{10}\right|+\left|\varepsilon_{11}\right|+\left|\varepsilon_{t}\right|\right)\left|H_{21}\right||\widetilde{t}|\right) / \widetilde{b} \leq 121 \varepsilon .
\end{array}
$$


We conclude that in any case

$$
a=\widetilde{a}\left(1+\varepsilon_{a}\right), \quad b=\widetilde{b}\left(1+\varepsilon_{b}\right), \quad\left|\varepsilon_{a}\right|,\left|\varepsilon_{b}\right| \leq 121 \varepsilon .
$$

This implies that, e.g.

$$
\begin{aligned}
& G_{21}=f l(-s n \sqrt{|a|})=\widetilde{G}_{21}\left(1+\varepsilon_{G}\right) \\
& \left|\varepsilon_{G}\right| \leq\left|\varepsilon_{s n}\right|+\left|\varepsilon_{a}\right| / 2+2 \varepsilon \leq 81.5 \varepsilon
\end{aligned}
$$

so that

$$
B=G=\widetilde{G}+\delta G, \quad|\delta G| \leq 81.5|\widetilde{G}| \varepsilon
$$

Thus,

$$
\begin{aligned}
B J B^{T} & =G J G^{T}=(\widetilde{G}+\delta G) J(\widetilde{G}+\delta G)^{T}=H+E \\
|E| & \leq 2 \cdot 81.5|\widetilde{G}||\widetilde{G}|^{T} \varepsilon \\
& =163|G||G|^{T} \varepsilon
\end{aligned}
$$

and (4.2.1) holds.

The induction step must also be done separately for a $1 \times 1$ and a $2 \times 2$ pivot. We can assume without loss of generality that $\bar{P}$ from (4.1.4) is the identity. Moreover, permuting the columns of $G$ in order to sort the elements of $J$ (see (4.1.9)) does not influence the statement of the theorem. From (4.1.4) and (4.1.11) we conclude that

$$
H=\left[\begin{array}{cc}
X & C^{T} \\
C & Y
\end{array}\right]=\left[\begin{array}{cc}
B & 0 \\
Z & I
\end{array}\right]\left[\begin{array}{cc}
J & 0 \\
0 & H_{1}
\end{array}\right]\left[\begin{array}{cc}
B^{T} & Z^{T} \\
0 & I
\end{array}\right] .
$$

Suppose that we do a $1 \times 1$ step, and that (4.2.1) holds for all matrices of order $n-1$. Then (4.2.11) holds with

$$
\begin{aligned}
B & =f l\left(\left|H_{11}\right|^{1 / 2}\right)=\left|H_{11}\right|^{1 / 2}+\delta B \\
|\delta B| & \leq\left|H_{11}\right|^{1 / 2} \varepsilon \\
Z & =f l(C J / B)=C J\left|H_{11}\right|^{-1 / 2}+\delta Z \\
|\delta Z| & \leq 2 \varepsilon|C|\left|H_{11}\right|^{-1 / 2}, \\
H_{1} & =f l\left(Y-Z J Z^{T}\right)=Y-Z J Z^{T}+F_{1}, \\
\left|F_{1}\right| & \leq 2 \varepsilon\left(|Y|+|Z||Z|^{T}\right) .
\end{aligned}
$$

The induction assumption implies that

$$
G_{1} J_{1} G_{1}^{T}=P_{1} H_{1} P_{1}^{T}+E_{1}
$$

where

$$
\left|E_{1}\right| \leq 136(n-1) \varepsilon\left(P_{1}\left|H_{1}\right| P_{1}^{T}+\left|G_{1}\right|\left|G_{1}\right|^{T}\right) .
$$


Now Alg. 4.1.1 yields

$$
G=\left[\begin{array}{cc}
B & 0 \\
P_{1} Z & G_{1}
\end{array}\right]
$$

so that

$$
G\left[\begin{array}{ll}
J & \\
& J_{1}
\end{array}\right] G^{T}=\left[\begin{array}{cc}
B J B^{T} & B J Z^{T} P_{1}^{T} \\
P_{1} Z J B^{T} & P_{1} Z J Z^{T} P_{1}^{T}+G_{1} J_{1} G_{1}^{T}
\end{array}\right] .
$$

Setting $P=I \oplus P_{1}$ and using (4.2.12), we obtain

$$
\begin{aligned}
G\left[\begin{array}{ll}
J & \\
& J_{1}
\end{array}\right] G^{T}= & P\left[\begin{array}{cc}
H_{11} & C^{T} \\
C & Y
\end{array}\right] P^{T} \\
& +\left[\begin{array}{cc}
2 \delta B J\left|H_{11}\right|^{1 / 2} & \delta C^{T} \\
\delta C & E_{1}+P_{1} F_{1} P_{1}^{T}
\end{array}\right](1+O(\varepsilon)) \\
& =H+E,
\end{aligned}
$$

where

$$
\delta C=P_{1}\left(\delta Z J\left|H_{11}\right|^{1 / 2}+C\left|H_{11}\right|^{-1 / 2} \delta B\right) .
$$

Using this and (4.2.12), we obtain

$$
|E| \leq\left[\begin{array}{cc}
2\left|H_{11}\right| & 3|C|^{T} P_{1}^{T} \\
3 P_{1}|C| & \left|E_{1}+P_{1} F_{1} P_{1}^{T}\right|
\end{array}\right] \varepsilon
$$

From (4.2.12) it follows that

$$
\left|H_{1}\right| \leq(1+2 \varepsilon)\left(|Y|+|Z||Z|^{T}\right) .
$$

By using (4.2.13), we have

$$
\left|E_{1}+P_{1} F_{1} P_{1}^{T}\right| \leq(136(n-1)+2)\left(P_{1}\left(|Y|+|Z||Z|^{T}\right) P_{1}^{T}+\left|G_{1}\right|\left|G_{1}\right|^{T}\right) \varepsilon .
$$

Inserting the above relation into (4.2.15) we obtain

$$
|E| \leq(136(n-1)+3)\left(P|H| P^{T}+|G||G|^{T}\right) \varepsilon,
$$

which, in turn, implies (4.2.1).

Now suppose that we do a $2 \times 2$ pivot, and that (4.2.1) holds for all matrices of order $n-2$. From the analysis of the $1 \times 1$ step, we see that we can without loss of generality assume that $P_{1}$ equals identity. Let $H$ be partitioned as in (4.1.4), and let $\widetilde{Q}^{T} X \widetilde{Q}=\widetilde{D}$ be the exact spectral decomposition of $X$. Let $Q$ and $D$ be the computed matrices $\widetilde{Q}$ and $\widetilde{D}$, respectively. The analysis of the $2 \times 2$ case for $n=2$ holds for the floating-point spectral decomposition of $X$, as well. Thus, (4.2.5) and (4.2.8) imply that

$$
\begin{array}{lll}
Q=\widetilde{Q}+\delta Q, & & |\delta Q| \leq 19|\widetilde{Q}| \varepsilon \\
D=\widetilde{D}+\delta D, & & |\delta D| \leq 121|\widetilde{D}| \varepsilon
\end{array}
$$


Now (4.2.11) holds with $H_{1}$ defined by (4.2.12), and $B$ and $Z$ as follows: from (4.2.9) it follows directly that

$$
\begin{aligned}
B & =f l\left(Q|D|^{1 / 2}\right)=\widetilde{Q}|\widetilde{D}|^{1 / 2}+\delta B \\
|\delta B| & \leq 81.5|\widetilde{Q}||\widetilde{D}|^{1 / 2} \varepsilon,
\end{aligned}
$$

and from (4.1.11) and (4.2.17) it follows that

$$
\begin{aligned}
Z & =f l\left(C Q|D|^{-1 / 2} J\right)=C \widetilde{Q}|\widetilde{D}|^{-1 / 2} J+\delta Z \\
|\delta Z| & \leq 83.5|C||\widetilde{Q}||\widetilde{D}|^{-1 / 2} \varepsilon .
\end{aligned}
$$

As in the $1 \times 1$ case, the induction assumption (4.2.13), where now

$$
\left|E_{1}\right| \leq 136(n-2) \varepsilon\left(\left|H_{1}\right|+\left|G_{1}\right|\left|G_{1}\right|^{T}\right),
$$

implies (4.2.14). This, (4.2.18), (4.2.19), (4.2.12), and (4.2.13), imply that

$$
G\left[\begin{array}{cc}
J & \\
& J_{1}
\end{array}\right] G^{T}=\left[\begin{array}{cc}
X & C^{T} \\
C & Y
\end{array}\right]+\left[\begin{array}{cc}
\delta X & \delta C^{T} \\
\delta C & E_{1}+F_{1}
\end{array}\right] \equiv H+E,
$$

where

$$
\delta C=\delta Z J|\widetilde{D}|^{1 / 2} \widetilde{Q}^{T}+C \widetilde{Q}|\widetilde{D}|^{-1 / 2} J \delta B^{T}
$$

From (4.2.10) it follows directly that

$$
|\delta X| \leq 163|B||B|^{T} \varepsilon
$$

As in the proof of (4.2.16), we have

$$
\left|E_{1}+F_{1}\right| \leq(136(n-2)+2)\left(|Y|+|Z||Z|^{T}+\left|G_{1}\right|\left|G_{1}\right|^{T}\right) \varepsilon
$$

and it remains to bound $|\delta C|$ from above. From (4.2.18) and (4.2.19) it follows

$$
|\delta C| \leq 165|C||\widetilde{Q}||\widetilde{Q}|^{T} \varepsilon
$$

It is easy to see that

$$
|C||\widetilde{Q}||\widetilde{Q}|^{T} \leq|C|+\left[\left|C_{\cdot 2}\right| \quad\left|C_{\cdot 1}\right|\right],
$$

where $C \cdot j$ denotes the $j$-th column of $C$. Further,

$$
\begin{aligned}
|Z||B|^{T} & =\left.|C \widetilde{Q}| \widetilde{D}\right|^{-1 / 2}+\delta Z|\cdot||\widetilde{D}|^{1 / 2} \widetilde{Q}^{T}+\delta B^{T} \mid \\
& \geq|C \widetilde{Q}||\widetilde{Q}|^{T}-165|C||\widetilde{Q}||\widetilde{Q}|^{T} \varepsilon .
\end{aligned}
$$

Now

$$
\left(|C \widetilde{Q}||\widetilde{Q}|^{T}\right)_{\cdot i}=\left|C \cdot{ }_{\cdot 1} \widetilde{c s}-C_{\cdot 2} \widetilde{s n}\right| \widetilde{c s}+\left|C_{\cdot 1} \widetilde{s n}+C_{\cdot 2} \widetilde{c s}\right||\widetilde{s n}|
$$


Simple checking of all possible combinations for the signs of $C_{i j}$ and $\widetilde{s n}$ shows that either

$$
\left|C_{i 1} \widetilde{c s}-C_{i 2} \widetilde{s n}\right|=\left|C_{i 1}\right| \widetilde{c s}+\left|C_{i 2}\right||\widetilde{s n}|
$$

or

$$
\left|C_{i 1} \widetilde{s n}+C_{i 2} \widetilde{c s}\right|=\left|C_{i 1}\right||\widetilde{s n}|+\left|C_{i 2}\right| \widetilde{c s} .
$$

If (4.2.24) holds for some $i$, then

$$
\left(|C \widetilde{Q}||\widetilde{Q}|^{T}\right)_{i 1} \geq\left|C_{i 1}\right|\left(\widetilde{c s}^{2}-\widetilde{s n}^{2}\right)+2\left|C_{i 2}\right||\widetilde{s n}| \widetilde{c s} .
$$

From (4.2.4) it follows that

$$
|\widetilde{t}| \geq \frac{1}{\alpha+\sqrt{1+\alpha^{2}}}
$$

Therefore,

$$
2 \widetilde{c s}|\widetilde{s n}| \geq\left(1+\alpha^{2}\right)^{-1 / 2} \geq 0.842
$$

and

$$
\left(|C \widetilde{Q}||\widetilde{Q}|^{T}\right)_{i 1} \geq 0.842\left|C_{i 2}\right|
$$

If (4.2.25) holds for some $i$, then

$$
\left(|C \widetilde{Q}||\widetilde{Q}|^{T}\right)_{i 1} \geq 2\left|C_{i 2}\right||\widetilde{s n}| \widetilde{c s}-\left|C_{i 1}\right|\left(\widetilde{c s}^{2}-\widetilde{s n}^{2}\right) .
$$

From (4.2.26) it follows that

$$
\widetilde{c s}^{2}-\widetilde{s n}^{2} \leq \alpha\left(1+\alpha^{2}\right)^{-1 / 2} \leq 0.54
$$

so that

$$
\left(|C \widetilde{Q}||\widetilde{Q}|^{T}\right)_{i 1} \geq 0.842\left|C_{i 2}\right|-0.54\left|C_{i 1}\right| .
$$

The similar analysis holds for the second column of $|C \widetilde{Q} \| \widetilde{Q}|^{T}$, too, and we conclude that

$$
\begin{aligned}
|C|+\left[\begin{array}{ll}
\left|C_{.2}\right| & \left.\left|C_{.1}\right|\right]
\end{array}\right] & \leq \frac{1}{0.842}|C \widetilde{Q}||\widetilde{Q}|^{T}+\left(1+\frac{0.54}{0.842}\right)|C| \\
& \leq 1.642\left(|C \widetilde{Q} \| \widetilde{Q}|^{T}+|C|\right)
\end{aligned}
$$

Using this and (4.2.23), and ignoring the relative error of $O(\varepsilon)$, we obtain

$$
\begin{aligned}
|\delta C| & \leq 165 \cdot 1.642\left(|Z||B|^{T}+|C|+165|C||\widetilde{Q}||\widetilde{Q}|^{T} \varepsilon\right) \varepsilon \\
& \leq 271\left(|Z||B|^{T}+|C|\right) \varepsilon .
\end{aligned}
$$

Finally, (4.2.1) follows by inserting this, (4.2.21) and (4.2.22) into (4.2.20), and the theorem is proved.

Q.E.D. 
Bunch [3] showed that the decomposition (4.1.3) with the unequilibrated diagonal pivoting (which is also used in Alg. 4.1.1) is stable in the following sense: let $L$ and $T$ be the factors of $H$ computed in floating-point arithmetic with precision $\varepsilon$. Then

$$
L T L^{T}=P H P^{T}+F,
$$

where

$$
\|F\|_{1} \leq \max _{k} \nu_{0}^{(k)}\left(21.6 n+7.9 n^{2}\right) \varepsilon,
$$

and $\nu_{0}^{(k)}$ is the value of $\nu_{0}$ in the $k$-th reduction step. The quantity $\max _{k} \nu_{0}^{(k)}$ is further bounded by

$$
\max _{k} \nu_{0}^{(k)} \leq \max _{i, j}\left|H_{i j}\right| 3.07 f(n) \sqrt{n}(n-1)^{0.446},
$$

where

$$
f(n)=\left(\prod_{k=2}^{n} k^{1 /(k-1)}\right)^{1 / 2} \leq 2 n^{(1 / 4) \log n} .
$$

The bound of Th. 4.2.1 compares favourably to the above bounds, since it does not contain the $n^{2} \varepsilon$ term. The quantity $\max _{k} \nu_{0}^{(k)}$ is implicitly included in the $|G||G|^{T}$ term of (4.2.1). Note that Th. 4.2.1 holds for a singular $H$, as well.

From the proof of Th. 4.2 .1 we see that $2 \times 2$ steps contribute much more to the error bound than $1 \times 1$ steps. If only $1 \times 1$ steps are performed (which is always the case when we decompose a positive definite matrix, and is often the case when we decompose scaled diagonally dominant matrices of [2]), then the bound (4.2.1) reduces to

$$
|E| \leq 3 n\left(P|H| P^{T}+|G||G|^{T}\right) \varepsilon .
$$

In the positive definite case Alg. 4.1.1 reduces to the Cholesky decomposition with complete pivoting, and only $1 \times 1$ steps are performed. The above inequality then implies

$$
\left|E_{i j}\right| \leq 6 n\left(\left(P H P^{T}\right)_{i i}\left(P H P^{T}\right)_{j j}\right)^{1 / 2} \varepsilon
$$

which is similar to the result of Demmel [9]. There the constant $6 n$ is replaced by $(n+1) /(1-(n+1) \varepsilon)$. Note, however, that the above bound holds for the outer product version of the Cholesky decomposition (Alg. 4.2.2 of [16]) with the addition of the complete pivoting, while Demmel [9] analysed the Gaxpy version (Alg. 4.2.1 of $[16])$.

Remark 4.2.2 Numerical experiments of Chap. 5 indicate that the bound (4.2.1) increases only slowly with $n$.

Remark 4.2.3 Other pivot strategies. Note that Th. 4.2.1 and then, in turn, Th. 4.3.1, hold for any pivot strategy for which (4.2.4) holds when we apply a $2 \times 2$ step. In particular, these theorems hold for the partial pivot strategy of [5] and for the 
pivot strategy given by Algorithm $\mathrm{C}$ of [4], which both require $O\left(n^{2}\right)$ search. We have chosen the unequilibrated diagonal pivoting since it has better bounds for the element growth, as well as the uniform upper bound for the scaled condition of the matrix $G^{T} G$ (see Sect. 4.4). Moreover, since the symmetric indefinite decomposition takes about $10 \%$ of the computing time, an $O\left(n^{3}\right)$ search, which is needed by the unequilibrated diagonal pivoting, does not considerably slow down the algorithm. However, theoretical and practical investigation of Algorithm C of [4] (for positive definite matrices this algorithm also reduces to Cholesky decomposition with complete pivoting), is certainly of interest.

$æ$ 


\subsection{Overall error bounds}

The results of the previous parts of the thesis suggest the following procedure to solve the real symmetric eigenvalue problem (4.1.1):

1. decompose $H$ as $H=G J G^{T}$ by first using Alg. 4.1.1 to obtain the decomposition (4.1.2), and then setting $G=P^{T} G$ as follows (in the notation of Alg. 4.1.1):

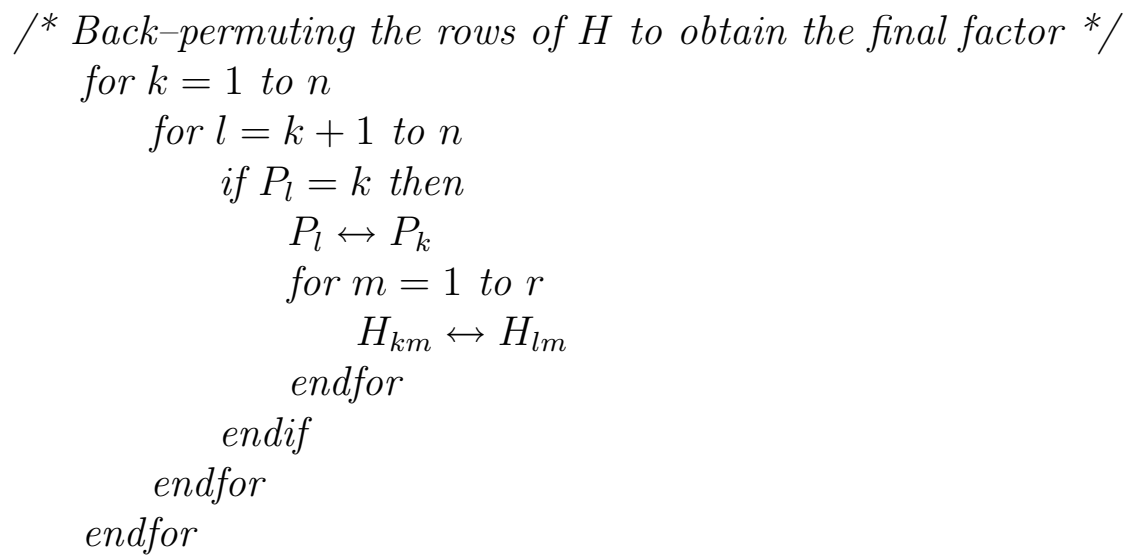

2. solve the problem (4.1.1) by applying any of the implicit $J$-orthogonal Jacobi methods of Chap. 3 on the pair $G, J$.

In this section we combine the error analysis of the symmetric indefinite decomposition, error analysis of the implicit $J$-orthogonal Jacobi methods, and the perturbation bounds of Chap. 2, to obtain error bounds for the computed eigensolution of the real symmetric eigenvalue problem. Bounds hold only in the non-singular case, since we cannot otherwise apply the perturbation theory of Sect. 2.2 to Th. 4.2.1. We give error bounds for the case when the implicit method of Alg. 3.3.1 is used. Error bounds for other implicit methods of Chap. 3 are obtained by simply substituting error bounds for those methods in the final estimate. We then show that an approximation for the error bounds can be obtained using only computed quantities. We also discuss what happens in the singular case. We give an interesting example how a change of the pivoting in the symmetric indefinite decomposition can considerably improve the accuracy of the obtained eigensolution. In the conclusion, we summarize some open problems.

Theorem 4.3.1 Let $H$ be a real symmetric non-singular matrix and let $\lambda$ be the $i$-th eigenvalue of $H$. Let $G, J$ be the decomposition of $H$ obtained by Alg. 4.1.1 in floatingpoint arithmetic with precision $\varepsilon$, and let $G=D_{G} B_{G}$, where $D_{G}$ is diagonal and the rows of $B_{G}$ have unit norms. Let $\lambda_{G}$ be the $i$-th eigenvalue of $G J G^{T}$. Let $G_{m}, J$ be the sequence of pairs obtained from the pair $G, J$ by Alg. 3.3 .1 in floating-point 
arithmetic with precision $\varepsilon$, and let $G_{M}, J$ be the final pair which satisfies the stopping criterion. For $m \geq 0$ write $G_{m}=B_{m} D_{m}$, where $D_{m}$ is diagonal and columns of $B_{m}$ have unit norms. Let $\lambda^{\prime}$ be the $i$-th calculated eigenvalue. Then, with the relative error of $O(\varepsilon)$, we have

$$
1-\eta-\eta_{1} \leq \frac{\lambda^{\prime}}{\lambda} \leq 1+\eta+\eta_{1}
$$

where

$$
\begin{aligned}
\eta & =\frac{272 n^{2} \varepsilon}{\lambda_{\min }\left(D_{G}^{-1}\left|G J G^{T}\right| D_{G}^{-1}\right)} \\
\eta_{1} & =2 \varepsilon \sum_{m=0}^{M-1} \frac{C_{m}}{\sigma_{\min }\left(B_{m}\right)}+n \cdot t o l+n^{2} \varepsilon
\end{aligned}
$$

I. Is the spectral absolute value defined in Sect. 2.1, and $C_{m}$ are constants from Th. 3.3.3.

Now suppose $\lambda$ is simple. Let $v$ be the corresponding eigenvector. Let $v^{\prime}$ be the eigenvector corresponding to $\lambda^{\prime}$, i.e. the $i$-th column of $G_{M}$ divided by its norm. Then

$$
\begin{aligned}
\left\|v^{\prime}-v\right\|_{2} \leq & \frac{\sqrt{2} \eta}{r g(\lambda)} \cdot \frac{1}{1-\left(1+\frac{1}{r g(\lambda)}\right) \eta} \\
& +\frac{4 \sqrt{2} \bar{\eta}}{r g_{G}\left(\lambda_{G}\right)} \cdot \frac{1}{1-\frac{3 \bar{\eta}}{r g_{G}\left(\lambda_{G}\right)}}+2 n \cdot t o l+n(3 n+4) \varepsilon,
\end{aligned}
$$

provided $1<(1+1 / \operatorname{rg}(\lambda)) \eta$ and $r g_{G}\left(\lambda_{G}\right)<3 \bar{\eta}$. Here $r g(\lambda)$ and $r g_{G}(\lambda)$ are defined by (2.2.29) and (2.3.1), respectively, and

$$
\begin{aligned}
\bar{\eta} & =\eta_{2}\left(2+\eta_{2}\right), \\
\eta_{2} & =\left(\eta_{1}+n \cdot \text { tol }+n^{2} \varepsilon\right) / 2,
\end{aligned}
$$

where $\eta$ is defined by (4.3.2).

Proof. From Th. 4.2.1, by multiplying (4.2.1) by $P^{T}$ from the left and by $P$ from the right, and then setting $G=P^{T} G$, it follows that

$$
H=G J G^{T}+\delta H,
$$

where

$$
|\delta H| \leq 136 n\left(|H|+|G||G|^{T}\right) \varepsilon
$$

Also,

$$
|H| \leq\left|G J G^{T}\right|+|\delta H| \leq|G||G|^{T}+|\delta H|
$$


so that, by ignoring the relative error of $O(\varepsilon)$, we have

$$
|\delta H| \leq 272 n|G||G|^{T} \varepsilon .
$$

Further,

$$
\begin{aligned}
\left|x^{T} \delta H x\right| & \leq|x|^{T}|\delta H||x| \leq 272 n|x|^{T}\left|D_{G} E D_{G}\right||x| \varepsilon \\
& \leq 272 n^{2} x^{T} D_{G}^{2} x \varepsilon \\
& \leq \frac{272 n^{2} \varepsilon}{\lambda_{\min }\left(D_{G}^{-1}\left|G J G^{T}\right| D_{G}^{-1}\right)} x^{T}\left|G J G^{T}\right| x .
\end{aligned}
$$

Applying Th. 2.2.1 to the pair $G J G^{T}, I$ with

$$
\eta \equiv \eta_{H}=\frac{272 n^{2} \varepsilon}{\lambda_{\min }\left(D_{G}^{-1}\left|G J G^{T}\right| D_{G}^{-1}\right)}, \quad \eta_{I}=0
$$

we obtain

$$
1-\eta \leq \frac{\lambda_{G}}{\lambda} \leq 1+\eta
$$

This and Cor. 3.3.4, by ignoring the relative error of $O(\varepsilon)$, imply (4.3.1).

Let $v_{G}$ be the eigenvector of $\lambda_{G}$. Applying (2.3.13) and Th. 2.2.13 to the matrix $G J G^{T}$ yields

$$
\left\|v_{G}-v\right\|_{2} \leq \frac{\eta}{r g(\lambda)} \cdot \frac{1}{1-\left(1+\frac{1}{r g(\lambda)}\right) \eta} .
$$

The relation (4.3.3) now follows from the above relation, (2.3.13), Th. 3.3.9, and the triangle inequality. The assumptions on $r g(\lambda)$ and $r g_{G}\left(\lambda_{G}\right)$ together with the proofs of Theorems 2.2.13 and 3.3.9, implies that $\lambda$ is throughout the algorithm well separated from the rest of the spectrum.

Q.E.D.

Remark 4.3.2 Th. 4.3.1 also holds if we substitute $\left|G J G^{T}\right|$ by $|H|$ in (4.3.2). Indeed, if we consider $G J G^{T}$ as $H-\delta H$, then

$$
\left|x^{T} \delta H x\right| \leq \frac{272 n^{2} \varepsilon}{\lambda_{\min }\left(D_{G}^{-1}|H| D_{G}^{-1}\right)} x^{T}|H| x \varepsilon,
$$

and we can apply Theorems 2.2.1 and 2.2.13 directly to $H$. We are using $\left|G J G^{T}\right|$ since $G$ is the computed factor and $D_{G}$ its exact scaling.

Th. 4.3.1 implies that the error bounds depend on how $D_{G}^{-1}$ scales $\mid G J G^{T} \mathbf{|}$. In the positive definite case $\mid G G^{T} \mathbf{|}=G G^{T}$ and the scaling with $D_{G}$ is optimal in the sense of (2.1.4). Our numerical experiments show that in the indefinite case the scaling with $D_{G}$ is also not far from the almost optimal one by $\left(\operatorname{diag}\left|G J G^{T}\right|\right)^{-1 / 2}$. 
It is natural to want to approximate the bounds (4.3.1) and (4.3.3) by using only computed quantities. We can substitute $r g(\lambda)$ and $r g_{G}\left(\lambda_{G}\right)$ with $r g\left(\lambda^{\prime}\right)$ and $r g_{G}\left(\lambda^{\prime}\right)$, respectively. Although $\lambda^{\prime}=\lambda(1+O(\varepsilon))=\lambda_{G}(1+O(\varepsilon))$, the above substitutions can have large relative errors. However, in numerical tests they are shown to be realistic. Further, we can substitute $\left|G J G^{T}\right|$ with $G_{M} G_{M}^{T}$. This is justified as follows: if $F$ is a $J$-orthogonal matrix which diagonalizes some $G^{T} G$ as in the proof of Th. 2.3.1, then $\left|G J G^{T}\right|=G F F^{T} G^{T}$. Now consider the matrix

$$
G_{M}^{\prime} \equiv\left(B_{M}+\delta B_{M}\right) D_{M} \equiv G_{M}+\delta B_{M} D_{M} \equiv\left(G+\delta G^{(M)}\right) R_{0} \cdot \ldots \cdot R_{M-1}
$$

from the proof of Th. 3.3.9. This matrix has orthogonal columns so that

$$
G_{M}^{\prime} G_{M}^{\prime T}=\mathbf{l}\left(G+\delta G^{(M)}\right) J\left(G+\delta G^{(M)}\right)^{T} \mathbf{I},
$$

and $G_{M}^{\prime} G_{M}^{\prime T}$ is, in turn, "not far" from $G_{M} G_{M}^{T}$. We have no theoretical results about the quality of this approximation, but its use is also justified by numerical experiments. Moreover, since we observed that the actual errors increase only slowly as $n$ and $M$ increase, and that the condition of the scaled matrix grows only little during the Jacobi process, we expect that

$$
\begin{aligned}
\left|\frac{\lambda^{\prime}-\lambda}{\lambda}\right| & \leq\left(\frac{1}{\lambda_{\min }\left(D_{G}^{-1} G_{M} G_{M}^{T} D_{G}^{-1}\right)}+\frac{2}{\sigma_{\min }(B)}\right) \varepsilon \\
\left\|v^{\prime}-v\right\|_{2} & \leq \frac{\eta}{r g\left(\lambda^{\prime}\right)} \cdot \frac{1}{1-\left(1+\frac{1}{r g\left(\lambda^{\prime}\right)}\right) \eta}+\frac{4 \bar{\eta}}{r g_{G}\left(\lambda^{\prime}\right)} \cdot \frac{1}{1-\frac{3 \bar{\eta}}{r g_{G}\left(\lambda^{\prime}\right)}},
\end{aligned}
$$

where

$$
\begin{aligned}
\eta & =\frac{\varepsilon}{\lambda_{\min }\left(D_{G}^{-1} G_{M} G_{M}^{T} D_{G}^{-1}\right)} \\
\bar{\eta} & =\frac{3 \varepsilon}{\sigma_{\min }(B)} .
\end{aligned}
$$

We cannot apply Th. 4.3.1 to singular matrices, since $\left|G J G^{T}\right|^{-1}$ is not defined. However, if we obtain a componentwise accurate factor $G$, as in the example (2.3.13), relative errors of the computed eigenvalues are bounded by Cor. 3.3.4. In this concrete example, we first have to bring $J$ to the form $I \oplus(-I)$. This is equivalent to performing $m$ trigonometric rotations for $\pi / 4$ on $G$ from the right. These rotations add $m$ terms to $\gamma$ of (3.3.20). Since Th. 3.3.9 (Th. 2.3.3) requires the non-singularity of $G$, we have no error bounds for the eigenvectors in this case.

The following example opens an interesting problem about the pivot choice in the symmetric indefinite decomposition. The example underlines once more the importance of exact factors, and shows what difficulties we have when trying to do deflation. Consider the matrix

$$
H=\left[\begin{array}{ccc}
1 & 1 & 1 \\
1 & 0 & 0 \\
1 & 0 & \alpha^{2}
\end{array}\right]
$$


where $\alpha>0$ is small. Alg. 4.1.1 decomposes $H$ as $H=G J G^{T}$ with

$$
G=\left[\begin{array}{lll}
1 & & \\
1 & 1 & \\
1 & 1 & \sqrt{\alpha^{2}-1+1}
\end{array}\right], \quad J=\operatorname{diag}(1,-1,1) .
$$

Since $|H|$ is given by (2.3.16), the error bounds of Th. 4.3.1 are large. Since in calculating $f l\left(\sqrt{\alpha^{2}-1+1}\right)$ we obtain only $\log \alpha^{2}-\log \varepsilon$ accurate digits, these error bounds are almost attained. However, since $1 / \sigma_{\min }(B) \approx 2.5$, any implicit Jacobi method will compute the eigensolution of the pair $G, J$ with high accuracy. This means that when using Alg. 4.1.1, we can do deflation only if the submatrix which is to be reduced at some stage is exactly zero. ${ }^{2}$ One way to accurately decompose $H$ is given by (2.3.15). Here we give another one: let us first choose $2 \times 2$ pivot in (4.1.5). Then we have

$$
H=\left[\begin{array}{lll}
1 & & \\
0 & 1 & \\
0 & 1 & \alpha
\end{array}\right]\left[\begin{array}{lll}
1 & 1 & \\
1 & 0 & \\
& & 1
\end{array}\right]\left[\begin{array}{lll}
1 & 0 & 0 \\
& 1 & 1 \\
& & \alpha
\end{array}\right]
$$

It is easy to see that with this pivot choice Alg. 4.1.1 returns the factor $G$ which has componentwise small relative errors. Therefore, the first terms of (4.3.1) and (4.3.3) are superfluous, and, since $1 / \sigma_{\min }(B) \approx 2$, the obtained eigensolution is accurate. This underlines the importance of accurate factors, and shows that the unequilibrated diagonal pivoting is not always the best choice.

Now we shortly summarize some open problems:

- finding a realistic upper bound for the growth of $1 / \sigma_{\min }\left(B_{M}\right)$,

- how well does $D_{G}^{-1}$ scale $\left|G J G^{T}\right|$, and how well does $\lambda_{\min }\left(D_{G}^{-1} G_{M} G_{M}^{T} D_{G}^{-1}\right)$ approximate $\lambda_{\min }\left(D_{G}^{-1}\left|G J G^{T}\right| D_{G}^{-1}\right)$

- proving Th. 2.3.3 for the non-square full column $\operatorname{rank} G$,

- improving the pivot strategy in Alg. 4.1.1, to avoid the unnecessary errors as in the example (4.3.5).

The last problem is very difficult, and it is similar to the problem of finding the best pivots in Gaussian elimination. It is easy to see (see also Rutishauser [23]) that Gauss' algorithm with complete pivoting is also inaccurate when applied to (4.3.5).

æ

\footnotetext{
${ }^{2} \operatorname{In}[6,5]$ deflation is also performed only in this case.
} 


\subsection{Bound for the scaled condition of $G^{T} G$}

The symmetric indefinite decomposition of Alg. 4.1.1 enables us to transform the eigenvalue problem (4.1.1) to the eigenvalue problem for the pair $G^{T} G, J$ on which we can use implicit methods of Chap. 3. In this section we show that the scaled condition of the matrix $G^{T} G$ is bounded by a function of $n$ irrespectively of the condition of the starting matrix $H$. This bound is nearly attainable. For related results see [19]. Numerical experiments of [13] and Chap. 5 show that the scaled condition of $G^{T} G$ is generally much smaller than our bound. This has a positive effect on the speed of the implicit methods applied on the pair $G^{T} G, J$. The results of this section are partially contained in [25].

For any positive definite matrix $H$ we define the scaled matrix $A \equiv \operatorname{Scal}(H)$ by $H=D A D$, where $D$ is diagonal positive definite, and $A$ has ones on the diagonal.

We analyse separately the positive definite and indefinite case. For $H$ positive definite Alg. 4.1.1 reduces to the Cholesky decomposition with complete pivoting (4.1.10), $P H P^{T}=L L^{T}$. Complete pivoting is equivalent to the fact that

$$
L_{i i}^{2} \geq \sum_{k=i}^{j} L_{j k}^{2}, \quad i=1, \ldots, n-1, \quad j>i .
$$

This implies

$$
L_{i i} \geq L_{j j}, \quad L_{i i}>\left|L_{j i}\right|, \quad i=1, \ldots, n-1, j>i
$$

Set

$$
H_{1}=L^{T} L
$$

If $(\lambda, x)$ is an eigenpair of $H$, then $\left(\lambda, L^{-1} x\right)$ is an eigenpair of $H_{1}$. Let $A_{1}=\operatorname{Scal}\left(H_{1}\right)$, i.e.

$$
H_{1}=D_{1} A_{1} D_{1}
$$

Demmel and Veselić [13] showed that $\kappa\left(A_{1}\right)$ is bounded by a constant depending only on the dimension $n$. For example, for $n=2$ it is easy to see that $\kappa\left(A_{1}\right)<3+2 \sqrt{2}$. For general $n$ their upper bound is

$$
\kappa\left(A_{1}\right)<e \cdot n \cdot n !
$$

which is, as they stated, a large overestimate. Here $e=\exp (1)$.

Now we analyse matrix $A_{1}$ in more detail and give a better bound which can be almost attained. We first illustrate the idea of the analysis on a $3 \times 3$ example. Let PHP $P^{T}=L L^{T}$ be the Cholesky decomposition with complete pivoting of a $3 \times 3$ positive definite matrix $H$. By

$$
D_{1}=\operatorname{diag}\left(\sqrt{L_{11}^{2}+L_{21}^{2}+L_{31}^{2}}, \sqrt{L_{22}^{2}+L_{32}^{2}}, L_{33}\right)
$$


we have

$$
A_{1}=\left[\begin{array}{ccc}
1 & \frac{L_{21} L_{22}+L_{31} L_{32}}{\sqrt{L_{11}^{2}+L_{21}^{2}+L_{31}^{2}} \sqrt{L_{22}^{2}+L_{32}^{2}}} & \frac{L_{31}}{\sqrt{L_{11}^{2}+L_{21}^{2}+L_{31}^{2}}} \\
1 & \frac{L_{32}}{\sqrt{L_{22}^{2}+L_{32}^{2}}} \\
\text { sym. } & & 1
\end{array}\right] .
$$

Now we need two monotonicity properties of the norm $\|\cdot\|_{2}$,

$$
\|A\|_{2} \leq\||A|\|_{2} \leq \sqrt{n}\|A\|_{2}
$$

where $|A|=\left|A_{i j}\right|$, and

$$
\left|A_{i j}\right| \leq B_{i j} \Longrightarrow\|A\|_{2} \leq\|B\|_{2} .
$$

From (4.4.5) and (4.4.1) we conclude that $\left\|A_{1}\right\|_{2} \leq\left\|A^{\prime}\right\|_{2}$ where $A^{\prime}=D^{-1}|L|^{T}|L| D^{-1}$, i.e. the worst case is when all $L_{i j}, i \neq j$, are non-negative. Treating $A_{23}^{\prime}$ as a monotonically increasing function of the (positive) variable $L_{32}$, from (4.4.1) it follows

$$
A_{23}^{\prime}<\frac{L_{22}}{\sqrt{L_{22}^{2}+L_{22}^{2}}}=\sqrt{\frac{1}{2}} .
$$

Treating $A_{13}^{\prime}$ as an increasing function of $L_{31}$ we have

$$
A_{13}^{\prime}<\frac{L_{11}}{\sqrt{L_{11}^{2}+L_{11}^{2}+L_{21}^{2}}} \leq \sqrt{\frac{1}{2}} .
$$

The element $A_{12}^{\prime}$ is an increasing function in three (positive) variables $L_{21}, L_{31}$ and $L_{32}$. Therefore,

$$
A_{12}^{\prime}<\frac{L_{11} L_{22}+L_{11} L_{22}}{\sqrt{L_{11}^{2}+L_{11}^{2}+L_{11}^{2}} \sqrt{L_{22}^{2}+L_{22}^{2}}}=\sqrt{\frac{2}{3}} .
$$

Finally, from (4.4.6) we conclude that

$$
\left\|A_{1}\right\|_{2}<\left\|\left[\begin{array}{ccc}
1 & \sqrt{2 / 3} & \sqrt{1 / 2} \\
\sqrt{2 / 3} & 1 & \sqrt{1 / 2} \\
\sqrt{1 / 2} & \sqrt{1 / 2} & 1
\end{array}\right]\right\|_{2} \leq 1+\sqrt{\frac{2}{3}}+\sqrt{\frac{1}{2}} .
$$

Further, we have $A_{1}^{-1}=D_{1} L^{-1} L^{-T} D_{1}$, where

$$
L^{-1}=\left[\begin{array}{lll}
1 & & \\
& 1 & \\
& & \frac{1}{L_{33}}
\end{array}\right]\left[\begin{array}{ccc}
1 & & \\
& \frac{1}{L_{22}} & \\
& -\frac{L_{32}}{L_{22}} & 1
\end{array}\right]\left[\begin{array}{ccc}
\frac{1}{L_{11}} & \\
-\frac{L_{21}}{L_{11}} & 1 & \\
-\frac{L_{31}}{L_{11}} & & 1
\end{array}\right] .
$$


From (4.4.5), (4.4.6) and (4.4.1) we see that $\left\|A_{1}^{-1}\right\|_{2}<\left\|D^{\prime} L^{\prime}\left(L^{\prime}\right)^{T} D^{\prime}\right\|_{2}$, where

$$
L^{\prime}=\left[\begin{array}{lll}
1 & & \\
& 1 & \\
& & \frac{1}{L_{33}}
\end{array}\right]\left[\begin{array}{ccc}
1 & & \\
& \frac{1}{L_{22}} & \\
& 1 & 1
\end{array}\right]\left[\begin{array}{ccc}
\frac{1}{L_{11}} & & \\
1 & 1 & \\
1 & & 1
\end{array}\right]
$$

and $D^{\prime}=\operatorname{diag}\left(\sqrt{3} L_{11}, \sqrt{2} L_{22}, L_{33}\right)$. Therefore

$$
D^{\prime} L^{\prime}\left(L^{\prime}\right)^{T} D^{\prime}=D^{\prime}\left[\begin{array}{ccc}
\frac{1}{L_{11}^{2}} & \frac{1}{L_{11} L_{22}} & \frac{2}{L_{11} L_{33}} \\
& \frac{2}{L_{22}^{2}} & \frac{3}{L_{22} L_{33}} \\
\text { sym. } & & \frac{6}{L_{33}^{2}}
\end{array}\right] D^{\prime}=\left[\begin{array}{ccc}
3 & \sqrt{6} & 2 \sqrt{3} \\
\sqrt{6} & 4 & 3 \sqrt{2} \\
2 \sqrt{3} & 3 \sqrt{2} & 6
\end{array}\right],
$$

and

$$
\left\|A_{1}^{-1}\right\|_{2}<\operatorname{Tr}(A)=13
$$

Alltogether we have

$$
\kappa\left(A_{1}\right)<13(1+\sqrt{2 / 3}+\sqrt{1 / 2}) \approx 32.81 .
$$

(The bound (4.4.4) for $n=3$ is $18 e \approx 48.96$.)

Theorem 4.4.1 Let $H$ be a real symmetric positive definite matrix of order $n$, and let $P H P^{T}=L L^{T}$ be its Cholesky decomposition with complete pivoting. Let $A_{1}=$ $D_{1}^{-1} L^{T} L D_{1}^{-1}$, where

$$
D_{1}=\operatorname{diag}\left(D_{1, i i}, \ldots, D_{1, n n}\right)=\operatorname{diag}\left(L^{T} L\right), \quad D_{1, i i}=\left(\sum_{k=i}^{n} L_{k i}^{2}\right)^{1 / 2} .
$$

Then

$$
\kappa\left(A_{1}\right)<\left(1+\sum_{i=1}^{n-1} \sqrt{\frac{i}{i+1}}\right) \sum_{i=1}^{n}\left(1+\frac{2^{2(i-1)}-1}{3}\right)(n+1-i) .
$$

Proof. Reasoning as we did in the $3 \times 3$ example, we conclude that $\left\|A_{1}\right\|_{2}<\left\|A^{\prime}\right\|_{2}$ where

$$
A^{\prime}=\left[\begin{array}{ccccc}
1 & \sqrt{\frac{n-1}{n}} & \sqrt{\frac{n-2}{n-1}} & \cdots & \sqrt{\frac{1}{2}} \\
1 & \sqrt{\frac{n-2}{n-1}} & \cdots & \sqrt{\frac{1}{2}} \\
& 1 & \cdots & \vdots \\
& & \ddots & 1
\end{array}\right] .
$$


Therefore

$$
\left\|A_{1}\right\|_{2}<1+\sum_{i=1}^{n-1} \sqrt{\frac{i}{i+1}}
$$

which proves the first part of (4.4.7). As in the $3 \times 3$ example, we also conclude that

$$
\left\|A_{1}^{-1}\right\|_{2}=\left\|D_{1} L^{-1} L^{-T} D_{1}\right\|_{2} \leq\left\|D^{\prime} L^{\prime}\left(L^{\prime}\right)^{T} D^{\prime}\right\|_{2},
$$

where

$$
\begin{aligned}
D^{\prime} & =\operatorname{diag}(\sqrt{n}, \sqrt{n-1}, \ldots, \sqrt{2}, 1) \\
L^{\prime} & =L^{(n)} L^{(n-1)} \cdot \ldots \cdot L^{(1)} \\
L_{j k}^{(i)} & = \begin{cases}1, & j=k \\
1, & k=i, j=i+1, \ldots, n, \\
0, & \text { otherwise } .\end{cases}
\end{aligned}
$$

For the elements of the matrix $L^{\prime}$ we now have

$$
L_{i j}^{\prime}= \begin{cases}1, & i=j, \\ 2^{i-1-j}, & i>j, \\ 0, & i<j .\end{cases}
$$

Set $B=L^{\prime}\left(L^{\prime}\right)^{T}$ and $C=D^{\prime} L^{\prime}\left(L^{\prime}\right)^{T} D^{\prime}=D^{\prime} B D^{\prime}$. Then

$$
\begin{aligned}
B_{i i} & =1+\sum_{j=1}^{i-1}\left(L_{i j}^{\prime}\right)^{2}=1+\sum_{j=1}^{i-1} 2^{2(i-1-j)}=1+\sum_{k=0}^{i-2} 2^{2 k} \\
& =1+\frac{2^{2(i-1)}-1}{3}, \quad i=1, \ldots, n,
\end{aligned}
$$

and

$$
\begin{aligned}
B_{i j} & =L_{i j}^{\prime}+\sum_{k=1}^{j-1} L_{i k}^{\prime} L_{j k}^{\prime}=2^{i-1-j}+\sum_{k=1}^{j-1} 2^{i-1-k} 2^{j-1-k} \\
& =2^{i-1-j}+2^{i+j-2}\left(\sum_{k=0}^{j-1} 2^{-2 k}-1\right) \\
& =2^{i-j}\left(\frac{1}{2}+\frac{2^{2(j-1)}-1}{3}\right), \quad i=1, \ldots, n ; i>j
\end{aligned}
$$

Of course, $B_{i j}=B_{j i}$. Furthermore,

$$
C_{i j}=B_{i j} \sqrt{n+1-i} \sqrt{n+1-j} .
$$

Finally, $\|C\|_{2}<\operatorname{Tr}(C)$ and the second part of (4.4.7) is proved.

Q.E.D. 
In Th. 4.4.1 we have essentially proved that for any positive definite matrix $H$, the value $\kappa\left(A_{1}\right)$ is smaller than the product $\left\|A^{\prime}\right\|_{2}\|C\|_{2}$. We must, however, emphasize that the second (dominant) part of (4.4.7) is a very good approximation for $\|C\|_{2}$ in the sense that for all $n$

$$
\left(\sum_{i=1}^{n}\left(1+\frac{2^{2(i-1)}-1}{3}\right)(n+1-i)\right) /\|C\|_{2}<1.0001
$$

We can further symplify the inequality (4.4.7) by bounding $\left\|A^{\prime}\right\|_{2}$ by $n$ and $\|C\|_{2}$ by

$$
\int_{1}^{n+1}\left(1+\frac{2^{2(x-1)}-1}{3}\right)(n+1-x) d x<\frac{1}{3}\left(n^{2}+\frac{1}{\ln ^{2} 4} 2^{2 n}\right),
$$

which yields

$$
\kappa\left(A_{1}\right)<\frac{n}{3}\left(n^{2}+\frac{1}{\ln ^{2} 4} 2^{2 n}\right) .
$$

We have experimentally observed that

$$
\frac{1}{3}\left(n^{2}+\frac{1}{\ln ^{2} 4} 2^{2 n}\right) /\|C\|_{2}<1.1708 .
$$

Now we show that the transition from the matrix $H$ to the matrix $L^{T} L$ cannot spoil the condition of the scaled matrix too much. We use the technique from [14]. Set $A=\operatorname{Scal}\left(P H P^{T}\right)=D^{-1} L L^{T} D^{-1}, B=D^{-1} L$, and $B_{1}=L D_{1}^{-1}$. Then $A=B B^{T}$, $A_{1}=B_{1}^{T} B_{1}$ and

$$
B_{1}^{-1}=D_{1} L^{-1}=D_{1} B^{-1} D^{-1} .
$$

From (4.4.1) for every $1 \leq j \leq i \leq n$ it follows

$$
\begin{aligned}
\left|D_{1} B^{-1} D^{-1}\right|_{i j} & =\sqrt{\frac{L_{i i}^{2}+L_{i+1, i}^{2}+\cdots+L_{n i}^{2}}{L_{j 1}^{2}+L_{j 2}^{2}+\cdots+L_{j j}^{2}}}\left|B^{-1}\right|_{i j} \\
& \leq \sqrt{n-i+1} \frac{L_{i i}}{L_{j j}}\left|B^{-1}\right|_{i j} \leq \sqrt{n-i+1}\left|B^{-1}\right|_{i j} .
\end{aligned}
$$

Thus, (4.4.5) and (4.4.6) imply

$$
\left\|B_{1}^{-1}\right\|_{2} \leq\left\|\left|D_{1} B^{-1} D^{-1}\right|\right\|_{2} \leq \sqrt{n}\left\|\left|B^{-1}\right|\right\|_{2} \leq n\left\|B^{-1}\right\|_{2},
$$

that is, $\left\|A_{1}^{-1}\right\|_{2} \leq n^{2}\left\|A^{-1}\right\|_{2}$.

The bound (4.4.7) is almost attained for the matrices of the form $H=L L^{T}$, where

$$
\begin{aligned}
& L=L_{0} D_{0}, \\
& D_{0}=\operatorname{diag}\left(1, s, s^{2}, \ldots, s^{n-1}\right) \text {, } \\
& \left(L_{0}\right)_{i j}=\left\{\begin{array}{cc}
1, & i=j, \\
-c, & i>j, \\
0, & i<j .
\end{array}\right. \\
& s^{2}+c^{2}=1 \text {. }
\end{aligned}
$$


These matrices are due to Kahan and are described in [16]. When $c \rightarrow 1$, then $H$ and $\operatorname{Scal}(H)$ both tend to singular matrices. Since $H_{1}=L^{T} L=D_{0} L_{0}^{T} L_{0} D_{0}$ and $L$ is itself the optimal Cholesky factor of $H$, we conclude that

$$
A_{1}=\operatorname{Scal}\left(H_{1}\right)=\operatorname{Scal}\left(L_{0}^{T} L_{0}\right)=D_{1}^{-1} L_{0}^{T} L_{0} D_{1}^{-1}
$$

where

$$
D_{1}=\operatorname{diag}\left(\sqrt{1+(n-1) c^{2}}, \sqrt{1+(n-2) c^{2}}, \ldots, 1\right) .
$$

It is easy to verify that $\lim _{c \rightarrow 1} A_{1}^{-1}=C$. Therefore, the quotient between the bound (4.4.7) and $\kappa\left(A_{1}\right)$ is in this case equal to $\left\|A^{\prime}\right\|_{2} /\left\|A_{1}\right\|_{2}$ which is smaller than the first part of (4.4.7) (smaller than $n$ ).

At the end we have to point out that, even though the bound of Th. 4.4.1 may seem pessimistic, experiments from Demmel and Veselic [13] and Chap. 5 show that $\kappa\left(A_{1}\right)$ is in practice considerably better than $\kappa(A)$ and, thus, the examples like that of Kahan are very rare. Moreover, for the matrices defined by (4.4.10) it is possible to obtain much better $\kappa\left(A_{1}\right)$. Since $H_{i i}=1$, the optimal Cholesky decomposition requires no pivoting. However, permuting the matrix $H$ so that e.g. $H_{n n}$ comes to the position $(1,1)$ does not contradict the complete pivoting and results in $\kappa\left(A_{1}\right)<n^{2}$. Demmel and Veselić [13] showed that for positive definite matrix $H$

$$
\lambda_{\min }(A) \leq \frac{H_{i i}}{\lambda_{i}} \leq \lambda_{\max }(A),
$$

where $A=\operatorname{Scal}(H), \lambda_{i}$ denotes the $i$-th eigenvalue of $H$, and $H_{i i}$ 's and $\lambda_{i}$ 's have the same ordering. This means that the diagonal entries of $H$ can differ from the eigenvalues only by factors bounded by $\kappa(A)$. Applying this result to $H_{1}=L^{T} L$, wee see that the Cholesky decomposition usually has rank-revealing property. The complete pivoting usually gives satisfactory results, but the choice of the optimal pivoting as in the above example in an open problem. For related results about the rank-revealing QR decomposition see [7].

The following theorem holds for a non-singular but possibly indefinite $H$ :

Theorem 4.4.2 Let $H$ be a nonsingular symmetric matrix and let $P H P^{T}=G J G^{T}$ be its decomposition. Let $\mu=2.781$ denote the maximal value of the quantities $\left|L_{i j}\right|$ from (4.1.8), and let $A_{1}=\operatorname{Scal}\left(G^{T} G\right)$. Then

$$
\kappa\left(A_{1}\right)<n(1+15 n) 3.781^{2 n} .
$$

Proof. From (4.1.9) it follows

$$
\begin{aligned}
\left\|A_{1}\right\|_{2} & =\left\|\operatorname{Scal}\left(G^{T} G\right)\right\|_{2}=\left\|\operatorname{Scal}\left(P_{1}^{T} \sqrt{\left|D_{1}\right|} L_{1}^{T} L_{1} \sqrt{\left|D_{1}\right|} P_{1}\right)\right\|_{2} \\
& =\left\|\operatorname{Scal}\left(\sqrt{\left|D_{1}\right|} L_{1}^{T} L_{1} \sqrt{\left|D_{1}\right|}\right)\right\|_{2} \\
& =\left\|\operatorname{Scal}\left(L_{1}^{T} L_{1}\right)\right\|_{2}=\left\|D^{-1} L_{1}^{T} L_{1} D^{-1}\right\|_{2},
\end{aligned}
$$


where $D$ is diagonal with elements $D_{i i}=\left(L_{1}^{T} L_{1}\right)_{i i}=\left(Q^{T} L^{T} L Q\right)_{i i}$. Note that in estimating $\left\|A_{1}\right\|_{2}$ and $\left\|A_{1}^{-1}\right\|_{2}$ we can without loss of generality assume that $P_{1}=I$. The matrix $A_{1}$ is positive definite and has unit diagonal, so that

$$
\left\|A_{1}\right\|_{2}<n .
$$

Further,

$$
\left\|A_{1}^{-1}\right\|_{2}=\left\|D L_{1}^{-1} L_{1}^{-T} D\right\|_{2}=\left\|D Q^{T} L^{-1} L^{-T} Q D\right\|_{2} .
$$

Now we shall maximize elements of the matrices $D$ and $Q^{T} L^{-1}$ and use the monotonicity properties of the norm $\|\cdot\|_{2}$ as we did in Th. 4.4.1. The elements of $L^{-1}$ are largest in modulus if all under-diagonal elements of $L$ are equal to $-\mu$. Let us denote this "maximal" $L^{-1}$ by $\bar{L}$. Then

$$
\bar{L}_{i j}= \begin{cases}1, & i=j, \\ \mu(1+\mu)^{i-1-j}, & i>j, \\ 0, & i<j .\end{cases}
$$

Now

$$
\left|Q^{T} L^{-1}\right| \leq\left|Q^{T}\right| \bar{L} \leq L^{\prime}
$$

where

$$
L_{i j}^{\prime}= \begin{cases}1+\mu, & i=j \\ \mu(2+\mu)(1+\mu)^{i-1-j}, & i>j \\ 1, & i=j-1 \\ 0, & i<j-1\end{cases}
$$

Element $D_{i i}$ is the norm of the $i$-th column of $L Q$. It is easy to verify that

$$
D_{i i}=\sqrt{1+L_{i+1, i}^{2}+\ldots L_{n i}^{2}}
$$

when the index $i$ corresponds to a $1 \times 1$ pivot, and

$$
\begin{aligned}
D_{i i} & =\sqrt{c s^{2}+s n^{2}+\left(L_{i+2, i} c s-L_{i+2, i+1} s n\right)^{2}+\left(L_{n i} c s-L_{n, i+1} s n\right)^{2}}, \\
D_{i+1, i+1} & =\sqrt{c s^{2}+s n^{2}+\left(L_{i+2, i+1} c s+L_{i+2, i} s n\right)^{2}+\left(L_{n, i+1} c s+L_{n i} s n\right)^{2}},
\end{aligned}
$$

when the indices $i, i+1$ correspond to a $2 \times 2$ pivot. Therefore, it is always $D_{i i} \leq D_{i i}^{\prime}$, where $D^{\prime}$ is diagonal matrix with elements

$$
D_{i i}^{\prime}=\sqrt{1+2(n-i) \mu^{2}} .
$$

Now we have

$$
\begin{aligned}
\left\|A_{1}^{-1}\right\|_{2} & =\left\|D Q^{T} L^{-1} L^{-T} Q D\right\|_{2} \leq\left\|D^{\prime} L^{\prime}\left(L^{\prime}\right)^{T} D^{\prime}\right\|_{2} \leq \operatorname{Tr}\left(D^{\prime} L^{\prime}\left(L^{\prime}\right)^{T} D^{\prime}\right) \\
& =\sum_{i=1}^{n}\left[1+(1+\mu)^{2}+\sum_{j=1}^{i-1}\left(\mu(1+\mu)^{(i-1-j)}(2+\mu)\right)^{2}\right]\left(1+2(n-i) \mu^{2}\right)
\end{aligned}
$$




$$
\begin{aligned}
& =\sum_{i=1}^{n}\left[1+\mu(2+\mu)\left((1+\mu)^{2(i-1)}-1\right)\right]\left(1+2(n-i) \mu^{2}\right) \\
& \leq \mu(2+\mu)\left(1+2 n \mu^{2}\right) \sum_{i=1}^{n}(1+\mu)^{2(i-1)} \\
& \leq\left(1+2 n \mu^{2}\right)(1+\mu)^{2 n}
\end{aligned}
$$

which completes the proof of the theorem.

Q.E.D.

Due to the fact that some of the worst cases assumed in the above proof are impossible, the statement of Th. 4.4.2 is an overestimate. Numerical experiments of Chap. 5 show that $\kappa\left(\operatorname{Scal}\left(G^{T} G\right)\right)$ is, as in the positive definite case, generally very small.

If $H$ is singular, then Alg. 4.1.1 returns an $n \times r$ matrix $G$ of the full column rank. The nature of the proof of Th. 4.4.2 implies that (4.4.11) holds in this case, too (and that even with better constants, since some summations have fewer terms).

æ 


\section{Chapter 5}

\section{Numerical experiments}

In this chapter, we present the results of our numerical experiments. Briefly, we tested the algorithm of Sect. 4.3 and verified that error bounds of that section held in all examples. The comparison of our algorithms with the $\mathrm{QR}$ and the standard Jacobi algorithm showed that our algorithms are uniformly more accurate. In fact, the performance is better than we were able to explain theoretically, both because we could observe little or no growth in actual errors for increasing dimension, and because of small values attained by $\max _{m} \kappa\left(B_{m}\right) / \kappa\left(B_{0}\right)$ during the Jacobi part. The relative errors in eigenvalues were given by (4.3.4) multiplied by small coefficients which increased only slowly with $n$. The norm errors in eigenvectors were smaller than those predicted by (4.3.4) by an order of magnitude .

Tests were performed using FORTRAN on an IBM RISC/6000. The arithmetic is IEEE arithmetic with machine precision $\varepsilon_{S} \approx 5.9604 \cdot 10^{-8}$ in single, and $\varepsilon_{D} \approx$ $1.1102 \cdot 10^{-16}$ in double precision. Overflow/underflow tresholds are approximately $10^{ \pm 38}$ in single, and $10^{ \pm 308}$ in double precision. The machine has a special multiplyand-add function, maf, which computes $a=b+c * d$ as a single instruction. In single precision, maf first computes $c * d$ in double precision, adds $b$, and then rounds $a$ back to single precision. For IEEE arithmetic with maf, the constants 272 and $C_{m}$ from (4.3.2) are somewhat, but not essentially, smaller.

In our tests we used five different algorithms:

JGJ - the symmetric indefinite decomposition of Alg. 4.1.1 followed by the standard implicit method of Alg. 3.3.1,

JGJF - the symmetric indefinite decomposition of Alg. 4.1.1 followed by the fast implicit method of Alg. 3.4.1,

JGJFS - the symmetric indefinite decomposition of Alg. 4.1 .1 followed by the fast implicit method with self-scaling rotations of Alg. 3.4.5,

JAC - the standard Jacobi algorithm (We used Alg. 3.1.1 with $J=I$. Then no hyperbolic rotations are performed and $H$ does not have to be positive definite.), 
SSYEV - LAPACK single precision routine which implements tridiagonalization followed by QR iteration.

In all three implicit Jacobis the diagonal was kept separately according to Alg. 3.3.7.

We tested the accuracy as follows: we considered real symmetric non-singular eigenproblems. We first solved every problem using JGJ and JAC in double precision. We assumed that the digits of the computed eigenvalues which overlap in those two algorithms are correct. We took the eigenvectors computed by JGJ as the ones of reference. Then we solved the same problem with the single precision versions of JGJ, JGJF and JGJFS, and compared the answers with the double precision solution to see if they were as accurate as predicted (which they were). We also compared the solutions obtained by SSYEV and the single precision version of JAC. Absolutely small eigenvalues computed by SSYEV were often of the wrong sign, indicating total loss of relative accuracy. All Jacobi algorithms used the stopping criterion tol $=n \cdot \varepsilon$ and the parallel cyclic pivot strategy of [24].

The rest of the chapter is organized as follows: we first discuss the test matrix generation. We then discuss accuracy of the computed eigensolutions. We make an interesting remark about the sensitivity of the QR and the standard Jacobi algorithms to the initial permutations of the input matrix. After that we discuss behaviour of $\lambda_{\min }\left(D_{G}^{-1} G_{M} G_{M}^{T} D_{G}^{-1}\right)$, growth of $1 / \sigma_{\min }\left(B_{m}\right)$ during the implicit Jacobi process, and behaviour of the diagonal in fast rotations. Finally, we discuss convergence rates.

Test matrix generation. We generated two types of random matrices. The first type is divided in several categories according to dimension $n, \kappa(\widehat{A})$ (where $\widehat{A}_{i i} \equiv 1$, so that $\kappa(\widehat{A})$ is at most factor $n$ from $C(A, \widehat{A})$ from $(2.2 .12)$ ), and $\kappa(H)$. We first describe the algorithm used to generate a random matrix from these parameters and then the sets of parameters used. All steps were preformed in double precision. Given $\kappa(\widehat{A})$, we generated a positive definite diagonal matrix $D$ whose entries' logarithms are uniformly distributed between $[-0.5 \log \kappa(\widehat{A}), 0.5 \log \kappa(\widehat{A})]$. On $D$ we applied five sweeps of random trigonometric plane rotations, thus obtaining matrix $A_{0}$. On $A_{0}$ we applied five sweeps of the "anti-Jacobi" method, thus obtaining matrix $\bar{A}$. This method, due to Veselić, consists of an iterative application of trigonometric plane rotations, $A_{m+1}=J_{m}^{T} A_{m} J_{m}$, where $J_{m}$ is obtained in the following manner: let

$$
\left[\begin{array}{ll}
a & c \\
c & b
\end{array}\right], \quad\left[\begin{array}{cc}
c s & s n \\
-s n & c s
\end{array}\right],
$$

be the pivot submatrices of $A_{m}$ and $J_{m}$, respectively. Then $c s=1 / h$ and $s n=-t / h$, where

$$
\zeta=\frac{2 c}{b-a}, \quad t=\frac{\operatorname{sign} \zeta}{|\zeta|+\sqrt{1+\zeta^{2}}}, \quad h=\sqrt{1+t^{2}} .
$$

The sequence of matrices obtained by the anti-Jacobi method converges to a matrix $A$ where $A_{i i} \equiv \operatorname{Tr} D / n$, i.e. $\kappa(\operatorname{Scal}(A))=\kappa(A)$. The convergence is very slow. It 
often required 50 or more sweeps for $n=30$. However, after five sweeps $\kappa(\bar{A})$ and $\kappa(A)=\kappa(D)$ differ by no more than $10 \%$. Given $\kappa(H)$, we generated a positive definite diagonal matrix $D_{1}$ whose entries' logarithms are uniformly distributed between $[-0.5 \log \kappa(H), 0.5 \log \kappa(H)]$, and formed a positive definite matrix $\bar{H}=D_{1} \bar{A} D_{1}$. We then calculated the eigendecomposition $\bar{H}=U^{T} \bar{\Lambda} U$ by our algorithm, and changed some randomly selected eigenvalues into negative ones, thus obtaining matrix $\Lambda$. Our random test matrix was then $H=U^{T} \Lambda U$.

The values for $\kappa(\widehat{A})$ were $10,10^{2}$ and $10^{3}$, the values for $\kappa(H)$ were $10^{2}, 10^{5}, 10^{9}$, $10^{14}$ and $10^{20}$, and the values for $n$ were $10,20,50,100$ and 200. This makes a total of $3 \times 5 \times 5=75$ different classes of matrices. In each class of dimension $n=10$ matrices we generated 500 random matrices, in each class of $n=20$ we generated 300 random matrices, in each class of $n=50$ we generated 200 random matrices, in each class of $n=100$ we generated 100 random matrices, and in each class of $n=200$ we generated 50 random matrices. This makes a total of 17250 different test matrices.

The second type of test matrices were block scaled diagonally dominant (b.s.d.d) matrices of Th. 2.2.7 generated according to two parameters, dimension $n$ and $\kappa(H)$. We first randomly generated number of diagonal blocks $2 \leq n_{b} \leq n-1$, and the size of the blocks. We then generated a random symmetric orthogonal matrix $A$ with this block structure (the elements outside blocks are 0 ), and formed matrix $\bar{A}=A+N$, were $N$ is a random symmetric matrix with $\|N\|_{2} \leq 0.5$. Given $\kappa(H)$, we generated a positive definite diagonal matrix $D$ whose entries' logarithms are uniformly distributed between $[-0.5 \log \kappa(H), 0.5 \log \kappa(H)] . D$ is constant on the blocks which correspond to the blocks of $A$, so that $A$ and $D$ commute. Finally, we formed our test matrix $H=D \bar{A} D$. As above, we have chosen $\kappa(H) \in\left\{10^{2}, 10^{5}, 10^{9}, 10^{14}, 10^{20}\right\}$ and $n \in\{10,20,50,100,200\}$. In each class of dimension $n=10$ and $n=20$ matrices we generated 100 random matrices, in each class of $n=50$ we generated 50 random matrices, in each class of $n=100$ we generated 30 random matrices, and in each class of $n=200$ we generated 10 random matrices.

Accuracy of the computed eigensolution. For every matrix we first calculated expected relative error in eigenvalues and expected norm error in eigenvectors according to (4.3.4) with $\varepsilon=\varepsilon_{S}=5.9604 \cdot 10^{-8}$. For every eigenvalue we calculated relative error

$$
\frac{\left|\lambda_{D, i}-\lambda_{S, i}\right|}{\left|\lambda_{D, i}\right|}
$$

where $\lambda_{D, i}$ denotes the $i$-th reference eigenvalue, and $\lambda_{S, i}$ denotes the $i$-th single precision eigenvalue. For every eigenvector we calculated the error $\left\|v_{D, i}-v_{S, i}\right\|_{2}$, where $v_{D, i}$ and $v_{S, i}$ are the eigenvectors corresponding to $\lambda_{D, i}$ and $\lambda_{S, i}$, respectively. Table 1 shows quotients of the maximum of the relative errors in single precision eigenvalues and the expected relative error of (4.3.4). For all quantities we give mean value, standard deviation, maximum and minimum attained on the respective 


\begin{tabular}{|r|r|r|c|c|c|}
\hline \multicolumn{5}{|c|}{ Table 1: } & $\max _{i}\left\{\left|\lambda_{D, i}-\lambda_{S, i}\right| /\left|\lambda_{D, i}\right|\right\}$ \\
\hline$n$ & & MEANected relative error & STD & MAX & MIN \\
\hline 10 & JGJ & 1.551 & 1.342 & 6.710 & .0676 \\
& JGJF & 1.562 & 1.372 & 7.658 & .0554 \\
& JGJFS & 1.225 & 1.024 & 6.347 & .0565 \\
\hline 20 & JGJ & 2.267 & 2.137 & 10.53 & .1105 \\
& JGJF & 2.330 & 2.199 & 10.32 & .1231 \\
& JGJFS & 1.618 & 1.509 & 8.216 & .0984 \\
\hline 50 & JGJ & 4.282 & 4.165 & 17.01 & .2256 \\
& JGJF & 4.355 & 4.282 & 18.34 & .2332 \\
& JGJFS & 2.737 & 2.625 & 11.14 & .1872 \\
\hline 100 & JGJ & 6.653 & 6.528 & 26.56 & .3609 \\
& JGJF & 6.803 & 6.721 & 27.45 & .3595 \\
& JGJFS & 4.191 & 4.168 & 20.06 & .2357 \\
\hline 200 & JGJ & 12.13 & 11.53 & 38.97 & .9087 \\
& JGJF & 12.26 & 11.60 & 39.11 & .9693 \\
& JGJFS & 7.546 & 7.239 & 25.62 & .5904 \\
\hline
\end{tabular}

class of test matrices. We see that the expectations were fulfilled up to a slowly growing constant, thus the statements of Remarks 3.2.6 and 4.2.2 that the actual errors increase only slowly as $n$ or $M$ increases. Note that the quotients in Table 1 increase at most linearly in $n$, which is still far below the theoretical growth of $O\left(n^{2}\right)$ from (4.3.2). Comparing the data for JGJ and JGJF indicates that the use of maf makes no difference in practice ( $m a f$ is theoretically fully exploited by fast rotations in JGJF, and only partially exploited in JGJ). Note that JGJFS is slightly more accurate than JGJ and JGJF.

Table 2 shows quotients of the maximum of the norm errors in single precision eigenvectors and the expected norm error. We see that the actual errors are considerably smaller than the expected ones, for which we have no explanation. Note, also, that the quotients are almost independent of $n$, and that JGJFS is now somewhat less accurate than JGJ and JGJF.

Table 3 shows quotients between maximal relative errors in eigenvalues of SSYEV (JAC) and JGJFS. We see that SSYEV and JAC often had no accurate digits, and are therefore unreliable. SSYEV and JAC performed as well or even slightly better than our algorithms on those matrices for which parameter $\kappa(H)$ was small, i.e. on the matrices where our perturbation theory and the standard one do not differ much.

Tables 1, 2 and 3 are obtained from the first type of test matrices. Data for b.s.d.d matrices are similar, except that JAC is for those matrices as accurate as our algorithms. 


\begin{tabular}{|r|r|r|r|r|r|}
\hline \multicolumn{6}{|c|}{ Table 2: $\frac{\max _{i}\left\|v_{D, i}-v_{S, i}\right\|_{2}}{\text { expected norm error }}$} \\
\hline$n$ & & MEAN & STD & MAX & MIN \\
\hline 10 & JGJ & .0144 & .0106 & .0895 & .0002 \\
& JGJF & .0147 & .0118 & .1258 & .0003 \\
& JGJFS & .0149 & .0111 & .0945 & .0003 \\
\hline 20 & JGJ & .0138 & .0120 & .1095 & .0008 \\
& JGJF & .0145 & .0133 & .1099 & .0009 \\
& JGJFS & .0159 & .0144 & .1112 & .0002 \\
\hline 50 & JGJ & .0168 & .0152 & .1056 & .0004 \\
& JGJF & .0181 & .0169 & .1018 & .0007 \\
& JGJFS & .0230 & .0232 & .1364 & .0014 \\
\hline 100 & JGJ & .0177 & .0175 & .1397 & .0008 \\
& JGJF & .0195 & .0197 & .1356 & .0010 \\
& JGJFS & .0285 & .0292 & .1938 & .0012 \\
\hline 200 & JGJ & .0198 & .0191 & .0808 & .0001 \\
& JGJF & .0231 & .0223 & .1045 & .0003 \\
& JGJFS & .0365 & .0349 & .1467 & .0011 \\
\hline
\end{tabular}

\begin{tabular}{|r|r|r|r|r|r|}
\hline \multicolumn{6}{|c|}{ Table 3: Quotients of maximal relative errors in eigenvalues } \\
\hline$n$ & & MEAN & STD & MAX & MIN \\
\hline 10 & SSYEV/JGJFS & $6.6 \cdot 10^{5}$ & $9.3 \cdot 10^{5}$ & $4.5 \cdot 10^{6}$ & .1687 \\
& $\mathrm{JAC} /$ JGJFS & $1.0 \cdot 10^{4}$ & $1.3 \cdot 10^{5}$ & $3.1 \cdot 10^{6}$ & .1055 \\
\hline 20 & $\mathrm{SSYEV} / J G J F S$ & $4.2 \cdot 10^{5}$ & $4.9 \cdot 10^{5}$ & $2.1 \cdot 10^{6}$ & .1812 \\
& $\mathrm{JAC} / \mathrm{JGJFS}$ & $4.8 \cdot 10^{4}$ & $1.6 \cdot 10^{5}$ & $1.2 \cdot 10^{6}$ & .1282 \\
\hline 50 & $\mathrm{SSYEV} / \mathrm{JGJFS}$ & $2.2 \cdot 10^{5}$ & $2.1 \cdot 10^{5}$ & $8.3 \cdot 10^{5}$ & .1136 \\
& $\mathrm{JAC} / \mathrm{JGJFS}$ & $1.2 \cdot 10^{5}$ & $1.9 \cdot 10^{5}$ & $7.6 \cdot 10^{5}$ & .1595 \\
\hline 100 & $\mathrm{SSYEV} / \mathrm{JGJFS}$ & $1.2 \cdot 10^{5}$ & $1.1 \cdot 10^{5}$ & $4.5 \cdot 10^{5}$ & .0631 \\
& $\mathrm{JAC} / \mathrm{JGJFS}$ & $1.0 \cdot 10^{5}$ & $1.1 \cdot 10^{5}$ & $4.5 \cdot 10^{5}$ & .1608 \\
\hline 200 & $\mathrm{SSYEV} / J G J F S$ & $4.1 \cdot 10^{4}$ & $4.4 \cdot 10^{4}$ & $1.4 \cdot 10^{5}$ & .0553 \\
& $\mathrm{JAC} / \mathrm{JGJFS}$ & $3.7 \cdot 10^{4}$ & $4.4 \cdot 10^{4}$ & $1.4 \cdot 10^{5}$ & .1877 \\
\hline
\end{tabular}




\begin{tabular}{|c|c|c|c|c|c|}
\hline & Table & $\lambda_{\min }$ & $\frac{\lambda_{\min }(\widehat{A}}{{ }^{1} G_{M} C}$ & $\overline{\left.D_{G}^{-1}\right)}$ & \\
\hline$n$ & & MEAN & STD & MAX & $\overline{\mathrm{MIN}}$ \\
\hline 10 & TYPE 1 & 1.216 & .2970 & 3.076 & 0.9166 \\
\hline & TYPE 2 & 2.742 & 1.249 & 6.000 & 1.100 \\
\hline 20 & TYPE 1 & 1.412 & .1665 & 4.411 & .9696 \\
\hline & TYPE 2 & 3.816 & 1.505 & 8.300 & 1.100 \\
\hline 50 & TYPE 1 & 1.821 & .6617 & 5.000 & 1.000 \\
\hline & TYPE 2 & 6.944 & 3.318 & 17.00 & 1.100 \\
\hline 100 & TYPE 1 & 2.347 & .9997 & 5.588 & 1.200 \\
\hline & TYPE 2 & 12.12 & 7.186 & 25.00 & 1.500 \\
\hline 200 & TYPE 1 & 3.522 & 1.654 & 7.272 & 1.608 \\
\hline & TYPE 2 & 20.85 & 8.900 & 37.00 & 6.500 \\
\hline
\end{tabular}

Remark. We have observed that the QR and the standard Jacobi algorithm often improved in accuracy when the starting matrix was permuted so that the symmetric indefinite decomposition needs no permutations. In many cases even the accuracy of our algorithms was achieved. This phenomenon in an interesting open problem, and can serve as an empirical advice to someone using QR or the standard Jacobi.

Behaviour of $1 / \lambda_{\min }\left(D_{G}^{-1} G_{M} G_{M}^{T} D_{G}^{-1}\right)$. Table 4 displays values of

$$
\frac{\lambda_{\min }(\widehat{A})}{\lambda_{\min }\left(D_{G}^{-1} G_{M} G_{M}^{T} D_{G}^{-1}\right)},
$$

where the denominator comes from (4.3.4), and $\widehat{A}=(\operatorname{diag}|H|)^{-1 / 2}|H|(\operatorname{diag}|H|)^{-1 / 2}$. We see that the quotients are small, thus implying that the errors induced by the symmetric indefinite decomposition satisfy the perturbation bounds of Sect. 2.2 almost optimally. The same values were obtained by all three of our algorithms. There are small differences between test matrices of the first and the second type.

Behaviour of $1 / \sigma_{\min }\left(B_{m}\right)$. Let $G_{m}=B_{m} D_{m}$ denote the sequence of matrices which was obtained by the implicit Jacobi from the starting pair $G_{0}, J$. As usual, the columns of $B_{m}$ have unit norms. Also, let $A_{m}=D_{m}^{-1} G_{m}^{T} G_{m} D_{m}^{-1}$. We calculated upper bounds for $\max _{m} \sigma_{\min }\left(B_{0}\right) / \sigma_{\min }\left(B_{m}\right)$ in two ways. Table 5 gives four values: SIGMA, HAD/SIGMA, BOUND and ROT. Here

$$
\operatorname{SIGMA}=1 / \sigma_{\min }\left(B_{0}\right), \quad \operatorname{HAD}=\left(\exp (1) / \operatorname{det}\left(A_{0}\right)\right)^{1 / 2} .
$$

BOUND and ROT were computed as follows: we computed a decreasing sequence $h_{m}$ as

$$
h_{0}=\mathrm{HAD}^{2} \text {, }
$$




$$
h_{m+1}=h_{m}\left(1-A_{m, i j}^{2}\right), \quad m \geq 0 .
$$

Each sweep of the parallel pivot strategy of [24] has $n$ parallel steps each having $p=(n-1) / 2$ rotations for $n$ odd, and $n-1$ parallel steps each having $p=n / 2$ rotations for $n$ even. We computed a non-decreasing sequence $s_{m}$ defined by

$$
\begin{aligned}
s_{0} & =\mathrm{SIGMA}^{2}, \\
s_{m} & =s_{m-1}, \quad m \geq 1, \quad m \bmod p \neq 0, \\
s_{m} & =s_{m-p}\left(1+\max _{0 \leq k \leq p-1}\left|A_{m-p+k, i j}\right|\right), \quad m \geq 1, \quad m \bmod p=0 .
\end{aligned}
$$

Recursive application of (3.2.34) implies that $1 / \sigma_{\min }^{2}\left(B_{m}\right) \leq s_{m}$. Recursive application of (3.2.36), together with (3.2.35), implies that $1 / \sigma_{\min }^{2}\left(B_{m}\right) \leq h_{m}$. Therefore, $1 / \sigma_{\min }^{2}\left(B_{m}\right) \leq \min \left\{s_{m}, h_{m}\right\}$ for every $m \geq 0$. Also, $s_{0} \leq h_{0}$. Let $m^{\prime}$ be the largest $m$ such that $s_{m} \leq h_{m}$. Then

$$
\operatorname{BOUND}=\left(s_{m} / s_{0}\right)^{1 / 2}, \quad \operatorname{ROT}=m^{\prime} .
$$

In other words, BOUND is the guaranteed upper bound for $\max _{m} \sigma_{\min }\left(B_{0}\right) / \sigma_{\min }\left(B_{m}\right)$.

The values of $1 / \sigma_{\min }\left(B_{0}\right)$ in Table 5 are very small, thus showing the non-trivial diagonalizing effect of the transition from matrix $H$ to pair $G^{T} G, J$. We also see that the guaranteed upper bound is reliable only for smaller dimensions, and that $s_{m}$ and $h_{m}$ usually meet in the first sweep. The data of Table 5 come from test matrices of the first type. Data for b.s.d.d matrices are similar.

A much better upper bound for $\max _{m} \sigma_{\min }\left(B_{0}\right) / \sigma_{\min }\left(B_{m}\right)$ was obtained by the algorithm of Sect. 3.2.2 (which, however, requires additional computational effort). This bound is by its nature always greater or equal $\sqrt{n}$, and the largest value attained in all experiments was $1.05 \sqrt{n}$. In fact, accuracy of computed eigensolutions implies that this is also an overestimate, that is, $1 / \sigma_{\min }\left(B_{m}\right)$ can grow only little before converging to 1 .

Behaviour of the diagonal in fast rotations. Table 6 shows four values: MINF is the smallest element of the diagonal of fast rotations obtained by JGJF, MINF/MINS is the quotient of this element and the smallest element of the diagonal of fast selfscaling rotations obtained by JGJFS, MAXF is the largest element of the diagonal of JGJF, and MAXF/MAXS is the quotient of this element and the largest element of the diagonal of JGJFS. We see that, even for large $n$, there is actually no danger of underflow/overflow.

Convergence rates. We compared computing times of JGJF and SSYEV, computing times of JGJ and JGJFS, and number of sweeps and rotations of JAC and JGJF. The speed ratio of JGJF and SSYEV is the following: for $n=200$, mean value, standard deviation, maximum and minimum are for matrices of the first type $(4.9,0.5,5.8,3.6)$, and for b.s.d.d matrices $(4.9,0.8,6.4,3.3)$. These ratios are realistic 


\begin{tabular}{|r|r|r|r|r|r|}
\hline \multicolumn{6}{|c|}{ Table 5: Behaviour of $1 / \sigma_{\min }\left(B_{m}\right)$} \\
\hline$n$ & & MEAN & STD & MAX & MIN \\
\hline 10 & SIGMA & 1.940 & .7408 & 5.193 & 1.032 \\
& HAD/SIGMA & 2.014 & 1.103 & 10.86 & 1.217 \\
& BOUND & 1.331 & .2513 & 2.877 & 1.649 \\
& ROT & 23 & 10 & 60 & 5 \\
\hline 20 & SIGMA & 2.813 & 1.249 & 9.481 & 1.130 \\
& HAD/SIGMA & 29.92 & 87.97 & 100.2 & 1.277 \\
& BOUND & 2.606 & 1.771 & 14.06 & 1.649 \\
& ROT & 85 & 28 & 170 & 10 \\
\hline 50 & SIGMA & 4.696 & 2.707 & 14.65 & 1.524 \\
& HAD/SIGMA & $1.0 \cdot 10^{10}$ & $1.4 \cdot 10^{11}$ & $2.9 \cdot 10^{12}$ & 2.182 \\
& BOUND & 330.1 & 784.8 & 550.5 & 1.649 \\
& ROT & 653 & 223 & 1175 & 75 \\
\hline 100 & SIGMA & 7.146 & 4.654 & 23.07 & 2.003 \\
& HAD/SIGMA & $3.2 \cdot 10^{31}$ & $6.9 \cdot 10^{32}$ & $1.4 \cdot 10^{34}$ & 57.41 \\
& BOUND & $4.1 \cdot 10^{9}$ & $8.6 \cdot 10^{10}$ & $1.8 \cdot 10^{12}$ & 9.220 \\
& ROT & 3247 & 1251 & 15500 & 105 \\
\hline
\end{tabular}

\begin{tabular}{|r|r|r|r|r|r|}
\hline \multicolumn{6}{|c|}{ Table 6: Behaviour of the diagonal in fast rotations } \\
\hline$n$ & & MEAN & STD & MAX & MIN \\
\hline 100 & MINF & .2839 & .1869 & .7100 & .0051 \\
& MINF/MINS & .4150 & .2584 & .9838 & .0086 \\
& MAXF & 1.323 & .1076 & 1.700 & 1.100 \\
& MAXF/MAXS & .9633 & .0767 & 1.230 & .7857 \\
\hline 200 & MINF & .0876 & .0855 & .3300 & .0005 \\
& MINF/MINS & .1418 & .1352 & .5409 & .0009 \\
& MAXF & 1.439 & .1158 & 1.900 & 1.300 \\
& MAXF/MAXS & 1.028 & .0827 & 1.357 & .9285 \\
\hline
\end{tabular}


although JGJF could be made slightly faster. Namely, SSYEV uses BLAS routines which are distributed together with RISC/6000 (and are therefore highly optimized), while our algorithm uses some extra BLAS type routines written by us (e.g. hyperbolic plane rotation).

Use of fast rotations, JGJFS, brought only about $5 \%$ speed up over JGJ.

We begin the comparison of sweeps and rotations needed for convergence of JAC and JGJF with a few details. JAC stopped when the last $n(n-1) / 2$ stopping tests $\left|H_{i j}\right| \leq t o l \sqrt{\left|H_{i i}\right|\left|H_{j j}\right|}$ succeeded. Since our implicit algorithms keep the diagonal in a separate vector, JGJF stopped after an empty sweep. Since one scalar product is needed to determine $\left(G^{T} G\right)_{i j}$ even if no rotation is performed, an empty sweep in JGJF requires approximately $1 / 3$ of the computation time of the full sweep, which is a slight dissadvantage. The symmetric indefinite decomposition used in JGJF amounts to no more than $2 / 9$ of one sweep and is neglected. Table 7 shows number of sweeps and rotations for JAC and JGJF, and quotient of numbers of rotations for JAC and JGJF.

We see that JAC needed averagely twice as much rotations as JGJF. Another important phenomenon, not readily seen in this table, is that number of rotations in JGJF is somewhat stable, that is, it did not depend much on parameters $\kappa(\widehat{A})$ and $\kappa(H)$, while in JAC number of rotations grew as $\kappa(H)$ grew. Data in Table 7 come from matrices of the first type. For b.s.d.d matrices, JAC performs better, that is, it needs averagely 1.5 times more rotations than JGJF.

æ 


\begin{tabular}{|r|r|r|r|r|r|}
\hline \multicolumn{7}{|c|}{ Table 7: Sweeps and rotations for JAC and JGJF } \\
\hline$n$ & & MEAN & STD & MAX & MIN \\
\hline 10 & SWEEP JAC & 5.1 & .96 & 9 & 3 \\
& ROT JAC & 166 & 30 & 257 & 105 \\
& SWEEP JGJF & 4.1 & .65 & 6 & 3 \\
& ROT JGJF & 107 & 30 & 191 & 43 \\
& JAC/JGJF & 1.6 & .4 & 4.2 & .98 \\
\hline 20 & SWEEP JAC & 7.2 & 1.7 & 12 & 4 \\
& ROT JAC & 935 & 191 & 1556 & 530 \\
& SWEEP JGJF & 4.8 & .72 & 7 & 3 \\
& ROT JGJF & 545 & 152 & 917 & 254 \\
& JAC/JGJF & 1.8 & .6 & 4.2 & 1.0 \\
\hline 50 & SWEEP JAC & 10.7 & 2.7 & 17 & 4 \\
& ROT JAC & 8305 & 1740 & 12719 & 4089 \\
& SWEEP JGJF & 5.7 & .92 & 8 & 4 \\
& ROT JGJF & 4317 & 1361 & 7427 & 2084 \\
& JAC/JGJF & 2.1 & .9 & 4.9 & .96 \\
\hline 100 & SWEEP JAC & 13.2 & 2.7 & 19 & 6 \\
& ROT JAC & 40431 & 10814 & 213460 & 19908 \\
& SWEEP JGJF & 6.5 & 1.1 & 9 & 5 \\
& ROT JGJF & 20502 & 7816 & 92408 & 9059 \\
& JAC/JGJF & 2.2 & 1.0 & 5.6 & .91 \\
\hline 200 & SWEEP JAC & 14.3 & 2.7 & 19 & 9 \\
& ROT JAC & 173952 & 25326 & 231892 & 135121 \\
& SWEEP JGJF & 8.0 & 1.3 & 10 & 6 \\
& ROT JGJF & 108607 & 31874 & 161841 & 57715 \\
& JAC/JGJF & 1.7 & .72 & 3.6 & .85 \\
\hline
\end{tabular}




\section{Bibliography}

[1] A. A. Anda, H. Park, Fast Plane Rotations with Dynamic Scaling, preprint, Computer Science Department, University of Minnesota, Minneapolis, 1990, to appear in SIAM J. Mat. Anal. Appl.

[2] J. Barlow, J. Demmel, Computing Accurate Eigensystems of Scaled Diagonally Dominant Matrices, SIAM J. Numer. Anal., Vol. 27, No. 3, (762-791) 1990.

[3] J. R. Bunch, Analysis of the diagonal pivoting method, SIAM J. Numer. Anal., Vol. 8, No. 4, (656-680) 1971.

[4] J. R. Bunch, L. Kaufmann, Some Stable Methods for Calculating Inertia and Solving Symmetric Linear Systems, Math. of Comp., Vol. 31, No. 137, (163-179) 1977.

[5] J. R. Bunch, L. Kaufmann, B. N. Parlett, Decomposition of a Symmetric Matrix, Numer. Math., No. 27, (95-109) 1976.

[6] J. R. Bunch, B. N. Parlett, Direct Methods for Solving Symmetric Indefinite Systems of Linear Equations, SIAM J. Numer. Anal., Vol. 8, No. 4, (639-655) 1971.

[7] T. F. Chan, Rank Revealing QR Factorizations, Linear Algebra and its Appl. 88/89, (67-82) 1987.

[8] A. Deichmöller, Über die Berechnung verallgemeinerter singulärer Werte mittels Jacobi-ähnlicher Verfahren, Dissertation, Fernuniversität, Hagen 1991.

[9] J. Demmel, On Floating Point Errors in Cholesky, LAPACK Working Note 14, Computer Science Dept. Report, University of Tennessee, Knoxville, October 1989 .

[10] J. Demmel, The inherent inaccuracy of implicit tridiagonal QR, IMA Preprint Series 963, University of Minnesota, Minneapolis, April 1992.

[11] J. Demmel, W. Gragg, Accurate Eigenvalues of Acyclic Matrices, Computer Science Division and Department of Mathematics preprint, University of California, Berkeley, 1992. 
[12] J. Demmel, W. Kahan, Accurate Singular Values of Bidiagonal Matrices, SIAM J. Sci. Stat. Comp., Vol. 11, No. 5, (873-912) 1990

[13] J. Demmel, K. Veselić, Jacobi's method is more accurate than QR, Computer Science Dept. Report 468, Courant Institute, New York, October 1989, to appear in SIAM J. Mat. Anal. Appl.

[14] Z. Drmač, unpublished manuscript, 1991.

[15] Z. Drmač, V. Hari, On quadratic convergence bounds for the J-symmetric Jacobi method, to appear in Numer. Math.

[16] G. H. Golub, C. F. Van Loan, Matrix Computations, University Press, Baltimore and London, 1989.

[17] K. P. Hadeler, Variationsprinzipien bei nichtlinearen Eigenwertaufgaben, Arch. Rat Mech. Anal., Vol. 30, (297-307) 1968.

[18] V. Hari, K. Veselić, On Jacobi methods for singular value decompositions, SIAM J. Sci. Stat. Comp., Vol. 8, (741-754) 1987.

[19] N. Higham, Analysis of the Cholesky decomposition of a semi-definite matrix, in Reliable Numerical Computation, Clarendon Press, eds. M. G. Cox and S. Hammarling, 1990.

[20] T. Kato, Perturbation Theory for Linear Operators, Springer, Berlin, 1966.

[21] R. Onn, A. O. Steinhardt, A. Bojanczyk, Hyperbolic Singular Value Decompositions and Applications, IEEE Trans. on Acoustics, Speech, and Signal Processing, (1575-1588) July 1991.

[22] B. Parlett, The Symmetric Eigenvalue Problem, Prentice Hall, Engelwood Cliffs, NJ, 1980.

[23] H. Rutishauser, Vorlesungen über numerische Mathematik, Vol. 1, Birkhäuser Verlag, Basel, Stuttgart, 1976.

[24] A. H. Sameh, On Jacobi and Jacobi-Like Algorithms for a Parallel Computer, Math. of Comp., Vol. 25, No. 115, (579-590) 1971.

[25] I. Slapničar, Upper bound for the condition of the scaled matrix of the symmetric eigenvalue problem, preprint, Seminarberichte aus dem Fachbereich Mathematik und Informatik Nr. 39, Fernuniversität, Hagen, 1990.

[26] I. Slapničar, On the growth of the condition of the scaled matrix in Jacobi methods, preprint, Seminarberichte aus dem Fachbereich Mathematik und Informatik Nr. 41, Fernuniversität, Hagen, 1992. 
[27] A. van der Sluis, Condition Numbers and Equilibration of Matrices, Numer. Math., Vol. 14, (14-23) 1969.

[28] K. Veselić, Jacobi's method is more accurate than QR II, unpublished manuscript, 1989.

[29] K. Veselić, An Eigenreduction Algorithm for Definite Matrix Pairs, preprint, FB Mathematik, Fernuniversität, Hagen, 1989, 1992, to appear in Numer. Math.

[30] K. Veselić, unpublished note, 1987.

[31] K. Veselić, V. Hari, A Note on a One-Sided Jacobi Algorithm, Numer. Math. 56, (627-633) 1989.

[32] K. Veselić, I. Slapničar, Floating-point perturbations of Hermitian matrices, preprint, FB Mathematik, Fernuniversität, Hagen, 1991, submitted to Lin. Alg. Appl.

[33] J. H. Wilkinson, The Algebraic Eigenvalue Problem, Clarendon Press, Oxford, 1965.

$æ$ 


\section{Lebenslauf}

13.07.1961

1967-1973

1973-1974

1974-1978

1978-1984

$1981-1984$

Juli 1984

$1984-1988$

$1985-1989$

Juli 1988

seit 1.06 .1990
Geburt in Split, Kroatien

Vater: Prof. Dr. Petar Slapničar

Mutter: Antonia Slapničar, geb. Jurić

Beruf des Vaters: Universitätsprofessor

Staatsangehörigkeit: kroatisch

Konfession: römisch-katholisch

Volksschule in Split

Volksschule in Palo Alto, Kalifornien

Gymnasium in Split

Studium der Mathematik an der Universität Zagreb

Studium des Klaviers an der Universität Zagreb

Hauptdiplom in Mathematik

Postdiplomstudium der Mathematik an der Universität Zagreb

Wissenschaftlicher Assistent an der Universität Split Magister der Naturwissenschaften aus dem Gebiet der Mathematik

Wissenschaftlicher Angestellter an der Fernuniversität Gesamthochschule Hagen

Hagen, den 29.05.1992

æ 